\title{
The Alterations of Tonus and Movements Through the Interplay Between the Cerebral Hemispheres and the Cerebellum 1,2
}

\author{
ELIZABETH C. CROSBY, RICHARD C. SCHNEIDER, BUD R. DE JONGE \\ AND PAUL SZONYI \\ Neurosurgical Research Laboratory, Division of Neurosurgery, Department \\ of Surgery, University of Michigan Medical Center, \\ Ann Arbor, Michigan
}

\begin{abstract}
This paper deals with the experimental production of involuntary movements and abnormal tonus in macaques (Macaca malatta) and their alterations in these animals and in children with cerebral palsy and other cerebral lesions.

The first major subdivision of the paper has three parts. The first part describes the effects of lesions in the macaque ccrebral hemispheres, ranging from a small destructive lesion in area 4 to an essentially complete bicortectomy. The case histories of a few patients document some of the results. The second part reports the effects of lesions in the macaque cerebellum ranging from small vermal injuries to complete cerebellectomies. The third part is concerned with successive lesions in the cerebellum and cerebral hemispheres of macaques and with planned cerebellar lesions in a few children with grave hypertonicity and marked involuntary movements. This subdivision is illustrated with photographs of the monkeys and the children at various stages of the procedures, photographs of many monkey brains at postmortem, and some photomicrographs showing lesions.

The second major subdivision has a discussion of the anatomic and the physiologic bases for the experimental results obtained and for the operations on the children. It correlates the material presented with data from the literature and is illustrated with photomicrographs of degenerated tracts and with diagrams. The paper stresses the balancing of cerebral hemisphere and cerebellar discharges in the regulation of tonus and in the stabilizing of movements. It discusses the possibility of producing a more effective tonus by making carefully planned lesions in cerebellar areas of animals or of children with highly handicapping hypertonicity.
\end{abstract}

\section{INTRODUCTION}

For over ten years the two senior authors and, of later years, the two junior authors as well have been studying, by experimental procedures on primates and in clinical cases, various alterations in tonus and the development of involuntary movements. This study has required a rather extensive consideration of the interrelations of various parts of the nervous system and an appreciation of the interplay of the discharges of certain brain regions with those of other parts of it. Partial substitution in function - such as that of an intact cortical area for certain activities of an injured cortical field - and the initiation of activity in one cortical region as a result of the firing from some distant cortical area have also had to be taken into account.

The fact that one is dealing with complex activities of the nervous system, that is, not merely with the functioning areas themselves but with the activities of interbalancing neuron arcs, has been increasingly apparent with the progress of the investigation. The tremendous importance of the higher centers as regulatory mechanisms of brain stem and spinal cord activities has also been emphasized by these studies.

Various reports (Schneider and Crosby, '54, '60, '63a, b; Crosby, '56) have been made and several lectures given by the senior members of the group during the progress of this work. Previously published work will be referred to as briefly as is con-

\footnotetext{
I This work was performed in the Kresge Neurosurgical Research Laboratory, University of Michigan Medical Center, Ann Arbor, Michigan with the support of the U.S. Public Health Service grant NB 03620-04 and a United Cerebral Palsy Association of Michigan Grant. These grants have been greatly appreciated.

Funds for publication of this manuscript have been provided by Mr. Alvin M. Bentley and by a grant from the Kresge Foundation. The authors wish to express their very sincere thanks for this aid.
} 
sistent with its correlation with the very much larger bulk of unpublished observations to be presented. Of course the present report leaves many questions unanswered.

\section{MATERIALS AND METHODS}

From a wide range of experimental and clinical material, the results of many experimental procedures and the case histories of several patients have been selected for presentation in this article, because they afford documentation of the material presented and of the viewpoints suggested. More than double the number of experiments on monkeys which are reported in this paper have been carried out and various other patients with symptoms similar to those described have been seen. A considerable number of the experimental results are not discussed because they are merely confirmatory. Certain experiments were unsuccessful, because the stimulations or the ablations were poorly located or because the animal succumbed before he could be properly tested.

Many of the animals were kept for considerable periods (in some cases for over three years) to demonstrate how much substitution of function occurred and what permanent behavioral changes resulted from the lesions. Many of the operations were additive. For example, tremor and/ or hypotonicity or hypertonicity were produced by one or more operations and then altered by further operative procedures. Certain animals, the brains of which were to be processed by one of several special techniques, were sacrificed at such times following the operations as were believed to be most advantageous for the technical procedures.

Preoperative and postoperative examinations were carried out. Detailed case histories were kept and are still available. Badly handicapped animals received excellent nursing care. For animals in which the cortex had been ablated bilaterally, a special, balanced diet, a hammock to prevent the development of sores due to pressure, frequent turning from side to side, baths, passive motion to prevent contractures, and other supportive methods were employed. The special walkers used for a monkey with a cerebellectomy and bilat- eral removal of the upper and the lower extremity regions of area 4 are shown in the figures (figs. 1 and 2). Monkeys which had had hemicortectomies or massive cerebellar lesions were removed from their cages frequently and encouraged in many ways to walk. Nursing care for the monkeys with extensive brain lesions and careful and frequent testing of all the animals are necessities if satisfactory observations and results are to be obtained.

After a few trial experiments had been made, ether was almost invariably used if stimulation was to be carried out. In some cases, where only ablations were contemplated, a barbiturate (usually Pentothal but occasionally Suritol) was used in dosages appropriate for the body weight of the animal. In some of the longer operations - and particularly in the cortectomies and the hemispherectomies - intravenous in jections of fluid were given during the operation. Normal saline or other fluids and penicillin were injected postoperatively after most major operations. The experiments were carried out under sterile neurosurgical technique.

In many cases, direct exposure of the operative site was obtained by a craniectomy. Stimulation of the areas thus exposed and stimulation and/or destruction of deep structures were carried out by use of the Grass stimulator (earlier, Type C; later, Type D). Stimulation and/or destruction of deep structures stereotactically were effected by use of the Chicago LabTronics apparatus. The strength of the current employed was usually between three and eight volts, the pulse frequency usually 40 , and the duration of the stimulus 1-5 msec.

The animals were sacrificed by administration of an overdose of ether or a barbiturate, followed by intravital injection of the fixative (such as $10 \%$ or $15 \%$ buffered formalin, or ammoniated alcohol) to be used in further processing. The brains were removed, the sites of the lesions were checked, and, in many cases, photographs were taken to document the findings. Various standard techniques were employed, such as Weil and thionin staining methods and Marchi, pyridine silver, and NautaGygax techniques, for preparation of the 

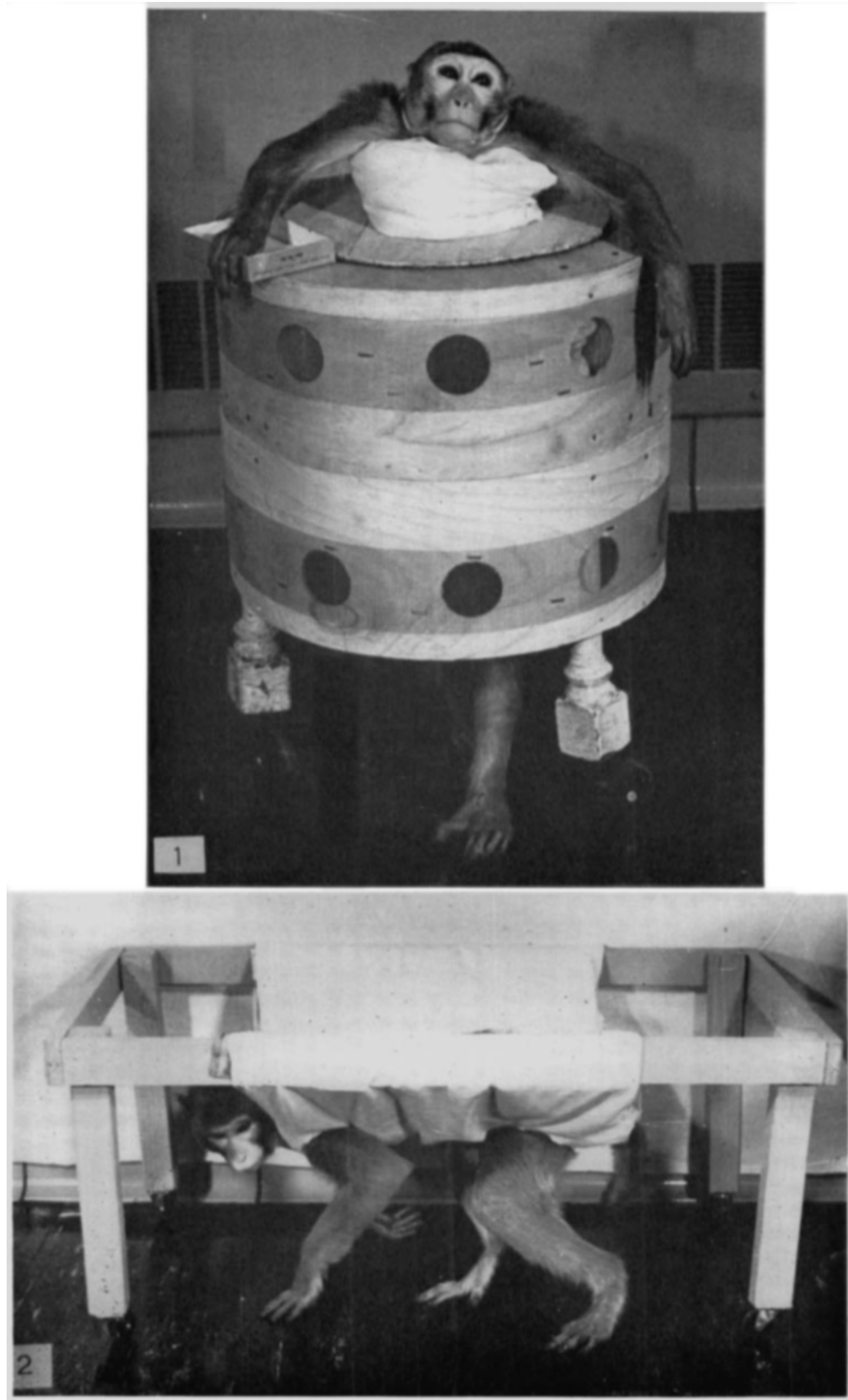

Fig. 1 Animal in barrel walker.

Fig. 2 Animal in canvas sling walker. 
microscopic material. Not all of these are illustrated.

The microscopic material was checked carefully to determine the location, the extent, and the character of the lesion. Often photomicrographs were made of informative sections.

For many of the monkeys and for the patients, frequent records of the behavioral changes, the motor deficits, the shifts in tonus, the presence or the absence of involuntary movements, and any other variations from the normal were acquired by use of the moving picture camera. It has been possible to obtain some "stills" from these records, but the authors have no satisfactory way to reproduce, in a published article, this most valuable part of the documentation of the results of the experiments on the monkeys or of the operations here reported on patients. Some photographs for publication have been taken with an ordinary camera.

\section{EXPERIMENTAL RESULTS}

\section{Lesions of cerebral hemisphere(s)}

The cortical lesions in this series of experiments range from destruction of a small part of area 4 to bilateral cortectomies. In some cases, the basal ganglia were included, wholly or in part, in the lesion. Many of the lesions were additive, beginning as small involvements of portions of one cortical field. Then the lesion was increased, area by area, until the total territory destroyed was very large. In other instances, a complete hemicortectomy or hemispherectomy was effected in one operation.

In the presentation which follows, the general effects of a particular type of lesion are given first, based on data accumulated from the records of all the monkeys in the series that had had this type of lesion. The documentation of the statements made follows (in small print) by presenting the actual case histories of one or more monkeys with such an injury. Supporting evidence is provided from photographs, photomicrographs, and "stills" from moving picture records.

\section{Lesions in area 4}

When area 4 is stimulated to establish the precise location of the various parts of the motor pattern and a lesion then placed in some special portion of the area (for example, the region from which, on stimulation, movements of the contralateral thumb or a sneer on the face on the side opposite to the excitation are produced), a slight hypotonia of the involved part of the face or body results (figs. $3 a$ and $b$ ). Such a hypotonia disappears within a short time of the operation, often within a day.

Following small lesions in area 4 which give contralateral extremity or face movements, there is a permanent loss of very precise movements, most noticeable when the finger, the toe, or the lip is involved. Complete destruction of area 4 on one side results in a contralateral hemiplegia with the loss of discrete, highly specialized movements such as bending of one finger. This is most marked in movements of the fingers and the toes. With bilateral removal of area 4 , a quadriplegia appears, with a permanent, bilateral loss of highly discrete motor performance and a difficulty in eating and in vocalization. Because of the difficulty in eating, in many bilateral ablations of area 4 , the face area was spared at least on one side.

In bilateral removals of area 4 , the tonus remains approximately normal, or slightly below normal, providing the ablation is superficial and no other areas are involved in the lesion. The animal walks and climbs very well again within a short time, although his hands and feet are paretic.

A bilateral frontoparietal craniectomy was performed on Monkey 5276, on November 10, 1960. The dura was retracted, the motor area identified, and the regions producing contralateral lower extremity, upper extremity, and face movements were established by stimulation. The face area was spared but the remainder of area 4 was undercut, bilaterally, in both the paracentral and the precentral regions.

Throughout the postoperative period, from November 12 to December 1, the animal showed a loss of precise, individual movements of the fingers and the toes. He walked with a shuffling movement of his feet but he climbed the cage walls fairly well. From the time he had recovered from the anesthetic, there was an approximately normal tonus in both upper and lower extremities and in the trunk. On December 1, 1960, area 6 was removed on the right (see p. 7).

\section{Lesions in motor and sensory strips}

If areas 3,1 , and 2 are destroyed unilaterally, a sensory loss appears on the side opposite the lesion. Although the threshold 

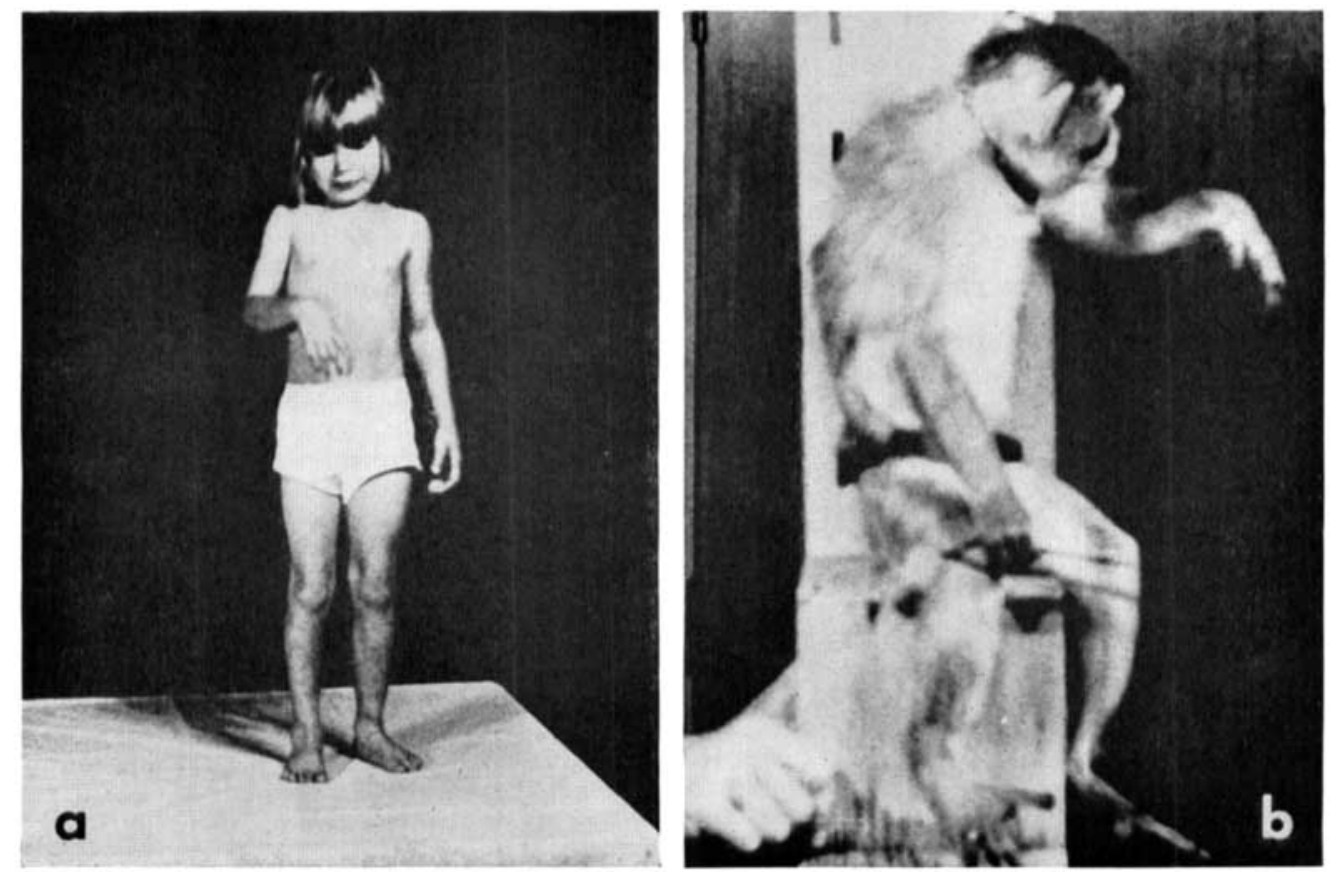

Fig. 3 (a) Flaccid monoplegia in a child resulting from a lesion in the forearm and hand regions of left area 4. (b) Comparable left monoplegia in monkey from a similar lesion in the right area 4. (Reprinted from the Proceedings of the Second International Meeting of Neurobiologists, Amsterdam, '59, by permission of the publishers, Elsevier Publishing Company - Schneider and Crosby, '60).

for painful and gross tactile stimuli may be slightly higher on the side contralateral to the lesion, the animal responds to both types of stimuli very readily after the operation, possibly suggesting that they may come into consciousness at subcortical levels. When an animal with this type of lesion handles an object there is, possibly, some evidence of a loss of discriminatory tactile sensibility in the hand contralateral to the lesion in the way he tends to feel the object with the intact hand. His loss of positional sense is evident not only in placing tests but also in his increased difficulty in climbing the cage. Such an animal frequently catches his hand or foot in the meshes of the cage and sometimes injures himself badly. Bilateral ablation of areas 3,2 , and 1 was distinctly disadvantageous to the animal.

A motor pattern, which may be regarded as an extension of that on area 4 (Penfield and Rasmussen, ' 50 ), or as evidence of the existence of a supplementary motor area in the somesthetic cortex (Foerster,
'31), is easily elicitable on stimulation of the sensory strip. It has been demonstrated by stimulation in the paracentral and the postcentral regions of many of the monkeys which have been studied (Crosby, Humphrey and Showers, '59). However, destruction of areas 3,1 , and 2 , without involvement of area 4 , produced no recognizable motor defects in our monkeys, a fact which may favor regarding the region as a second motor (or supplementary motor, or extrapyramidal) area as Foerster has done (see also p. 55).

On May 25, 1958, a bilateral craniectomy was performed on a female monkey (4806) which had been normal in respect to motor power and tonus on preoperative testing. The areas along each fissure were exposed and the regions giving leg and foot movements were identified by stimulation in each paracentral area and were destroyed bilaterally. The lesion was carried back behind each motor area to involve the adjoining somesthetic cortex.

The following day, an inability to carry out discrete movements with the toes, a paretic position of the feet when at rest, and a loss of positional sense in both feet were very evident. The loss of positional sense was highly disadvantageous to 
the animal in walking and climbing. The loss of fine tactile sensibility was not demonstrated satisfactorily. There was evidence of a slight hypertonicity in the involved extremities as late as July 1, 1958. Gradually, during July, the tonus became approximately normal but the sensory loss and the motor deficits were unchanged (fig. 4). On July 22 , a right hemispherectomy was carried out on this animal.

A similar operation was performed on Monkey 4755 with confirmation of these results. However, Monkey 4755 had less hypotonia and this hypotonia disappeared earlier.

On February 8, 1958, the areas on each side of each central fissure giving leg and foot movements were identified by stimulation and destroyed in Monkey 600. A slight hypotonicity of the legs and the feet was demonstrable immediately postoperatively but disappeared rapidly. Testing, on March 5, showed an animal with a normal tonus but inability to place satisfactorily with the feet and so a demonstrable loss of positional sense. On November 18, 1958, the motor and the somesthetic fields for face and upper extremity areas on each side of each central fissure were identified and destroyed bilaterally. In the immediate postoperative period, the monkey had a mild increase in tonus in all extremities which, however, soon became normal. Fine movements of the hands and around the mouth were lacking and there were obvious sensory deficits, which showed particularly in the loss of positional sense on the upper extremities. There was

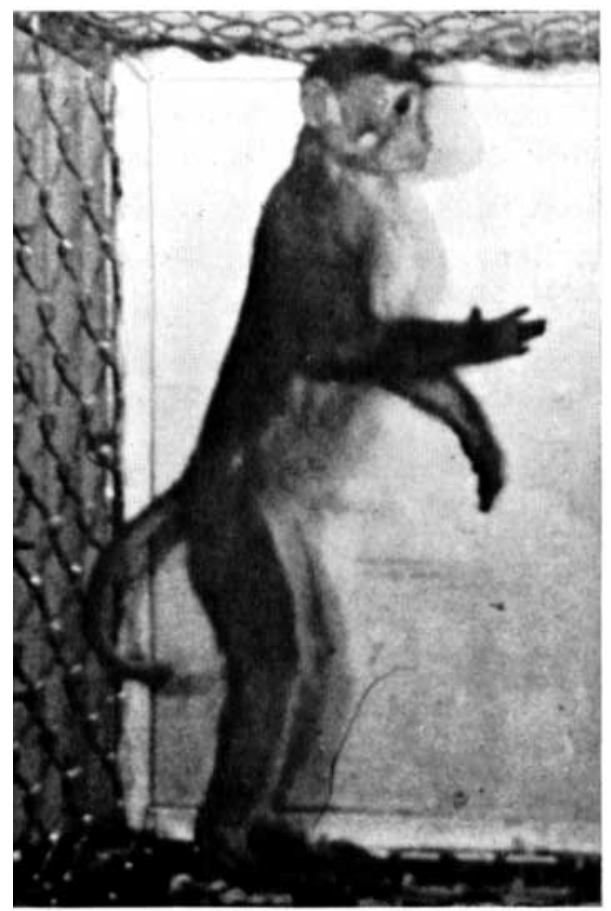

Fig. 4 Monkey 4806, July 17, 1958. an inability to place with the four extremities. The animal had difficulty in eating and swallowing because of the lesions in the face areas of the cortex. No other lesion was placed and, on December 14,1958 , the animal was sacrificed and the extent and the positions of the lesions verified in postmortem material.

Bilateral removal of the motor and the somesthetic areas along the central fissure in the regions verified by stimulation to be related to upper and lower extremity movements was carried out on Monkey 732, on November 7, 1957. The motion pictures taken on November 14, 1957 showed a somewhat belligerent animal that was able to walk and to jump up on the cage readily, but that could not hang onto the meshes with his normal dexterity. There was, possibly, a very slight increase in tonus in the lower extremities. On November 21, 1957, the animal had a left subtotal hemispherectomy (p. 36 ).

On November 20, 1958, the arm and the leg areas on either side of the left central fissure were identified by stimulation and destroyed in Monkey 4929. The lesion was continued down to the corpus callosum on the medial hemisphere wall and invaded area 5 directly posterior to the main lesion. Postoperatively, the animal had a motor deficit on the side opposite the lesion, most evident in the loss of fine movements of the fingers and the toes, a loss of positional sense on the involved limbs, and a mild hypotonicity. The sensory loss was permanent. The tonus rapidly became normal. By December 1, 1958, the animal could walk and climb quite well. He had, however, a tendency to hold his affected arm in extension as he sat in the cage. Discrete, individual movements of the fingers were no longer possible and he was unable to recognize the position of these digits. The animal died of diarrhea on December 6, 1958. The locations of the lesions were verified in the postmortem material.

\section{Lesions involving areas 4 and 6}

Since it has sometimes been proposed that hypertonicity results from the addition of lesions of area $4 \mathrm{~s}$ and/or area 6 to injuries in area 4 , unilateral and bilateral ablations were made in all three areas in several macaques. Some of these ablations were carried back into the somesthetic cortex.

The results may be summed up by stating that the tonus tended to be slightly below normal in animals with one-sided involvements but was either approximately normal or slightly and transiently decreased in animals with bilateral lesions. A grasp reflex (fig. 14) could be demonstrated postoperatively if area 6 was involved bilaterally but often could not be elicited when only one area 6 was ablated (see Travis, '55). 
The operated animals showed a lack of fine movements of the extremities (particularly of their distal parts) but they could walk and climb. There was a loss of positional sense demonstrable on the extremities contralateral to the lesion if this lesion involved the somesthetic cortex. The amount of the loss was consonant with the precise location and extent of the lesion. There may have been some lowering of discriminatory tactile sensibility contralateral to the involvement more readily demonstrable on the hand. However, none of the tests on tactile discrimination were entirely unequivocal.

In Monkey 2003, a left craniectomy was performed on November 4, 1954. The dura was reflected and areas 4 and 6 were stimulated to identify the regions giving upper extremity movements. These regions were ablated. Quite possibly area $4 \mathrm{~s}$ was invaded by the lesion. Postoperatively the monkey showed a loss of fine movements of the fingers on the side opposite to the lesion and a hypotonicity in the distal part of the upper extremity on this same side.

On November 11, 1954, a similar lesion was placed in the right hemisphere, the region to be ablated being determined again by stimulation. Following this second lesion, the tonus was still below normal in both upper extremities. Individual finger movements were impossible on either hand.

The animal lived for six months after the last operation. His status remained unchanged, except that the hypotonicity lessened and the tonus was approximately normal. Examination of the brain suggested that there had been a slight abrasion of the somesthetic cortex in the arm area on the left, as well as the intended bilateral lesions in areas 4 and 6 .

Monkey 5276, that has been reported on (p. 4) as having undergone a bilateral ablation of the upper and lower extremity regions of area 4, was reoperated on December 1,1960 . At this later date, area 6 was also removed on the right. Postoperatively, there were no recognized changes in the animal's status nor did it change during the next two months, up to the time that he was released to another experimenter. No grasp reflex was demonstrated, although Travis reported ('55) a slight transient grasp reflex on the removal of one premotor area in the monkey. The deficits from the earlier operation remained but his tonus was normal.

\section{Lesions in cortical areas other than area 4}

In order to assess the motor performance, after lesions in supplementary motor or extrapyramidal areas but in the presence of intact areas 4 , a series of small ablations in various cortical regions was carried out. The results varied depending upon the site of the lesion.

On November 4, 1955, the skull was trephined on the left and the temporal lobe exposed in Monkey 2013. The rostral end of the lobe and the superior temporal gyrus were spared but the remainder of the temporal cortex was removed. There were no overt changes in behavior. One week later, by a similar procedure, the corresponding areas of the right temporal lobe were removed and an attempt made to ligate the right anterior choroidal artery. The day following the operation the animal showed some posturing of his extremities and possibly a slight increase in tonus. These signs of possible basal ganglion involvement persisted for at least three weeks.

The animal had been borrowed for the experiments and was returned to his owner following tests on December 8, 1955. However, reports were received of the postmortem examination of the brain when the animal was sacrificed. This examination indicated that the division of the anterior choroidal artery had not been successful. There was some hemorrhage into the striatum.

In a series of monkeys used for demonstration of the homolateral and contralateral face movements obtainable on stimulation of the rostral part of the temporal lobe (Schneider and Crosby, '54), only a slight hypotonicity, or possibly a nor' mal tonus, was present after a one-sided lesion and perhaps a slightly increased tonus after bilateral ablations. The movements obtained on stimulation of this temporal area and the slight hypertonicity present on bilateral destruction have been verified in various experiments. The movements elicitable throughout the extent of the temporal lobe have been reported by Dr. DeJonge in a separate publication.

In 1955 , the results of a series of experiments dealing with parietal lobe stimulations to determine the patterns of movements clicitable from supplementary motor areas 5 and 7 were published by Fleming and Crosby ('55). Excision of areas 5 and 7 resulted in a slight transient hypotonia and some impairment, which was also transient, of the use of the contralateral extremities in the performance of movements. At the time the series was done, this was spoken of as "a slight transient impairment of the voluntary use of the contralateral arm and leg." In retrospect it seems probable that this was a sensory apraxia due to the destruction of the sensory association arcs in the parietal cortex.

A lesion was made in the lower half of area 6 on the lateral surface of the left hemisphere in a female macaque, Monkey 5406, on August 2, 1961. Postmortem verification of the site of the lesion indicated that it extended also slightly ventral to the arcuate fissure. The animal recovered rapidly from the operation, moving around the cage freely on the same day. On August 3, no deficit in motor power, no abnormalities in tonus, and no grasp reflex could be elicited. However, not all of the upper extremity area had been involved in the lesion. There was an inability to close the eye on the side of the lesion due quite certainly to the 
extension of the lesion beyond area 6. During the succeeding two weeks the status of the animal remained unchanged.

On August 17, 1961, a lesion was placed in the face area of the cortex at the base of the parietal fissure on the right, the area being identified by stimulation. No observed changes in tonus, behavior, or motor power followed this lesion. The animal was sacrificed September 6, 1961, and the site of the lesion verified.

\section{Hemicortectomies in monkeys}

All of the monkeys with total or subtotal hemicortectomies had a homonymous hemianopia on the side opposite the lesion, so that objects brought into the lateral side of the visual field contralateral to the cortectomy did not produce head and eye deviations even when the monkeys responded to stimulation of the visual field on the side of the lesion by turning the head or eyes in that direction. It was not always possible to obtain satisfactory results on every examination of each monkey because sometimes the animal would not cooperate. In some monkeys, the loss of following eye movements away from the side of the lesion was demonstrated, but again, not all monkeys could be interested in the procedure. With few exceptions, if the eyes were watched during a unilateral removal of the frontal cortex, they deviated toward the side of the lesion. Sometimes, because of the exigencies of the operation this reaction was not noted. As the monkey recovered from the anesthetic, following a hemicortectomy, such eye deviation was often present and often persisted postoperatively for a time.

Monkey 4806, that underwent a right hemispherectomy, on July 22, 1958, showed deviation of the eyes toward the side of the lesion on July 25. Monkey 3516 had a tendency to keep his eyes turned toward the left following a left cortectomy and this response was still evident two weeks after the experimental procedure although he could readily turn his eyes in all directions if he wished to do so. Monkey 4981 , semicomatose for several days after a left hemicortectomy on May 4, 1959, had a postoperative forced deviation of the eyes toward the left; however, this deviation was not evident on May 15, when the animal's condition was such as to warrant an extensive examination.
Without exception, all of the monkeys which suffered subtotal or total hemicortectomies had a loss of positional sense on the side opposite the lesion. Such an animal had an inability to place. His difficulty in learning to walk again was markedly due to a lack of knowledge of the location of his extremities, since he improved more rapidly after the examiner showed his foreleg to him and persuaded him to walk with it as he tried to move forward on four feet. Some monkeys quite definitely watched the involved forefoot as they tried to walk (for example Monkey 4981). The way the monkey placed his paretic hand and foot on the cage meshes so that a finger or toe was caught and often abraided may be regarded as another example of a sensory deficit on the appropriate side.

The monkeys tested after hemicortectomies responded to superficial and deep pain and gross tactile stimuli on either side of the body, although the responses to such stimulation were a trifle slower on the side contralateral to the lesion in some of these animals. It is possible that this slowing was due to the motor loss on the side opposite the lesion. The present experimenters, however, are not inclined to accept this as the only explanation since a monkey with a lesion only in one somesthetic cortex did not react quite so readily (particularly if the stimuli were not very strong) to painful or tactile excitation on the side contralateral as on the side homolateral to the cortical destruction. In a few cases some evidence was obtained that suggested that, although tactile sensibility was retained bilaterally after hemicortectomies, there was some lack in tactile discrimination. A monkey that was really curious about some object would always pick it up with the hand on the side of the lesion (that is, the unaffected hand), although before the operation he had showed no handedness. It seems probable that this was largely due to his motor deficit in the contralateral upper limb. However, after the object was in his unaffected hand, if he were sufficiently curious, he might not only look at it and perhaps taste and/or smell it but he might also rub it between his fingers. With the object in the affected hand, the monkey made no at- 
tempt to examine it until he had transferred it to the other hand. The proof for loss of tactile discrimination in these hemicortectomized monkeys is certainly equivocal.

Occasionally, after a hemicortecomy, a monkey acted as if he did not recognize his extremities on the side opposite the lesion as belonging to himself. Such a monkey often had more than the usual difficulty in learning to walk again although he made excellent progression movements. Monkey 2007 illustrated this curious condition which represents a kind of loss of body scheme. In the two monkeys where this condition was better recognized, it was not possible to determine whether it had any relation to handedness. Many, but not all, of our monkeys appeared to be ambidextrous. In man, the inability of a patient to recognize a part of his body as belonging to himself (although he may identify it as a hand or foot) is regarded as indicative of a lesion in the parietal association cortex usually of the nondominant (Critchley, '53) but sometimes of the dominant (Nielsen, '58) hemisphere.

All of the monkeys with a hemicortectomy had a loss of highly specialized, discrete movements, particularly evident in the performance of the individual fingers and toes. When such a monkey had a small object placed in his affected hand, he did not grasp it between his thumb and index finger but closed all, or nearly all, the fingers of his hand about it. In climbing, he grasped the cage wires with all of his fingers in a combined and relatively gross movement. Although, after a onesided cortectomy or hemispherectomy, a monkey was not able to stand, he was usually pulling himself around the cage within a day or so after the operation, standing upright by leaning with the unimpaired side of the body against the cage wall, and attempting to climb within a week, or less, postoperatively, if his recovery were not hindered by infection, hemorrhage, or some unrelated illness. A hemicortectomized or hemispherectomized monkey learns to walk again and to climb very well, and within a surprisingly short time, but none of those studied in this series ever regained individual finger and toe movements or recovered from his sensory disabilities. When the animal tried to walk or climb, the fingers and the toes flattened out (or curled against the wire), a condition which persisted for several years. Sometimes, a reflex very like the Babinski was obtained, in hemicortectomized monkeys, on the paretic lower extremity but it was not always possible to elicit it. The affected hand or foot was often held in a paretic pose, when the animal was at rest, even though he walked and climbed quite well.

All of the animals with hemicortectomies (like monkeys with unilateral frontal ablations) showed circling or repetitive movements. Monkeys kept for a long time after the cortex had been removed on one side still exhibited stereotyped responses typical of frontal lobe involvement. Monkey 5398 who had had a cortectomy on August 8, 1961 and was kept without further operation until August 7, 1963 still showed, at this later date, the typical repetitive and stereotyped movements characteristic of a frontal lobe ablation.

After a unilateral cortectomy, a monkey still makes sounds that presumably are his means of communicating his sentiments about a given situation to his fellow monkeys and probably to the world in general. Such speech, and studies by those competent to judge state that the monkey has speech (Lockard, '48), may be largely emotional in type, with bilateral representation in the cortex and projection to lower centers over corticohypothalamic and extrapyramidal systems. The crying and the movements sometimes obtained, in response to painful stimulation, from a bilaterally decorticate monkey or from a child with generalized cortical deficiency undoubtedly require only intact arcs from the thalamus through the hypothalamus or the basal ganglia to produce them.

Very shortly after a hemicortectomy, a monkey may have an essentially normal tonus or may show a slight hypotonicity. However, when in addition to the removal of the cortex there is an involvement of the lenticular nucleus, some increase of tone is usually demonstrable. If the lenticular lesion is quite large, clasp-knife rigidity (or hypertonicity suggesting it) often develops. 
Where one caudate nucleus is involved to any considerable extent, head deviation occurs. If the caudate on the other side is injured in the same operation, there is no deviation of the head, then, to either side. The head deviation is of fairly short duration. With caudate injuries there is a tendency toward hypotonicity, but where the destruction of the caudate is only a part of the experimental picture this hypotonicity may not appear.

On April 29, 1958, the skull of Monkey 4755 was trephined and the dura retracted to reveal the paracentral, the precentral, and the postcen. tral gyri in each hemisphere. The regions were stimulated to verify the positions of areas giving lower extremity movements and such areas were ablated with a sucker. The animal had an uncomplicated recovery. Postoperatively, she walked around the cage easily although her toes were unduly straight. She climbed less well because of the bilateral loss of individual toe movement on the hindlegs and a handicapping loss of positional sense on the lower extremities, especially on the feet. The inability to place with the feet was demonstrated. No abnormalities of tonus were noted.

On June 26, 1958, the opening in the skull was further enlarged to reveal area 6 and the dorsal part of the prefrontal area on the left. The motor pattern on area 6 was demonstrated and area 6 was ablated on both the medial and the lateral surfaces of the left hemisphere. The lesion extended a short distance rostral to area 6 into the prefrontal area. Immediately postoperatively, the animal showed some shock effects but recovered rapidly from the procedure. She was hyperkinetic, exhibited marked curiosity, and showed a tendency to perform stereotyped movements - all signs of frontal lobe involvement. No change in tonus or in her previous motor and sensory handicaps was noted, except a slight tendency to posture with the right hindleg. This might be interpreted as a motor apraxia, due to involvement of area 6 . There was no grasp reflex.

On July 11, 1958, the skull opening was again enlarged in Monkey 4755 and the dura resected to reveal the medial surface and the dorsal part of the lateral surface of area 5 , bilaterally. The cortical areas 5 were stimulated to delimit the regions from which lower extremity movements had been reported (Fleming and Crosby, "55) and these regions were ablated with a sucker. Following this ablation the animal was in excellent condition. She was still hyperkinetic, although perhaps not quite so overactive as before this last procedure. She showed more posturing of the lower extremities, particularly of the right hind limb, than before with the general appearance of increased tonus. Possibly a parietal lobe apraxia had also been added to her other deficits. The motor and the sensory losses incurred from the first experiments of course still persisted. Nevertheless, the animal walked quite well and climbed all around the cage. During the period between
July 12 and September 30 , she was tested repeatedly. Immediately after the operation, the tonus may have been slightly above normal in the lower extremities but, by July 17 , it was essentially normal and remained so up to September 30 .

On September 30,1958, the skull opening was carried farther away from the midline on the left, in order to expose the arm and the face areas of area 4 on that side. These regions were identified by stimulation and ablated. Postoperatively, the animal showed a right central facial paresis (which implies a motor weakness of the lower part of the face) and a paresis of the right upper extremity, in addition to the earlier deficits. The right upper extremity had a typical paretic pose and fine, individual finger movements were not possible on the right. No increase in tonus was noted. The animal moved around the cage and still climbed the cage walls although less well than did the unoperated animals.

On October 6, 1958, the skull was reopened on the right to reveal right area 4 . The region from which upper extremity movements were obtainable on stimulation was ablated. Postoperative testing showed a paretic left upper extremity having a loss of fine movements, along with the defects accumulated from the earlier operations.

On November 27, 1958, a left craniectomy was performed, the dura retracted and the remaining cortex removed from the left hemisphere. Although there were evidences of shock following this procedure, within two days she was trying to move her extremities even though she was prone on the cage floor. By four days after the hemicortectomy, the monkey was attempting to climb the cage wall. However, when removed from the cage, she fell as she tried to walk. The inability to walk appeared to be due particularly to her loss of positional sense on both lower extremities and the right upper extremity, so that she was not able to place her feet properly for maintaining an upright position. With a little help and a little practice she learned to walk within ten days of the last experimental procedure, although she had a tendency to walk with her left side close to the wall. Perhaps this was due to her tendency to circle toward that side which was marked after the hemicortectomy. She had evident piloerection over the shoulders.

When tested, the animal showed a right homonymous hemianopia. Light reflexes were normal. She had a right hemiplegia involving trunk and extremities, the lower part of the face and the one side of the tongue. She also had a paresis of the left lower extremity. There was a slight overactivity of the deep reflexes in the knees, more particularly in the knee on the left, but otherwise there were no overt evidences of increased tone except a certain stiffness in pose suggesting hypertonicity in the legs. The hand and the feet were held in a paretic pose when the animal was not moving about the cage. When rechecked on January 7 , the animal showed little change except some passive resistance to movement of the left lower extremity at the hip as well as at the knee. This waxed and waned and it was difficult to be sure that it was not due to uncooperativeness on the part of the animal. She continued to be hyper- 
kinetic and given to repetitive movements although she gradually circled less unless excited or angry.

During the period between January 7 and May 5,1959 , the animal was checked frequently. She remained in good general health and gradually stabilized. The piloerection over the shoulders still was evident and the monkey still performed stereotyped movements if excited but she was less irritable than earlier. The same motor and the sensory deficits were present as on January 7 . She was still unable to perform individual movements of the involved fingers and toes. The deep reflexes were increased in both legs and in the wrist on the right and there was some evidence of a right wrist drop. The tail was extended. There was a slight extensor tonus at the knee and the ankle on the right although none could be demonstrated on the left. The pose indicated some extensor tonus. It is to be remembered that at this time the animal had lesions in both hemispheres, greater on the left than on the right. On May 5, 1959, further operative procedures were carried out on this monkey.

On May 12, 1959, a left craniectomy was performed on a previously unoperated male monkey (Monkey 4981) and all the cortex removed from the left hemisphere except for small remnants in the precommissural septal area and in the hippocampal gyrus. Although the animal was awake that night he was still obtunded the next day. At that time he was hemiparetic on the right and held the right lower extremity extended and the right upper arm flexed. There was a further minor increase in tone. There was a loss of positional sense, as evidenced by his inability to place on the right, and a right homonymous hemianopia. A tactile loss was hard to assess. Light reflexes were normal.

By May 15, 1959, the animal was beginning to drag himself around the cage by his arms and was trying to feed himself. The extension of the right lower extremity and the flexion of the right upper extremity were no longer evident and there was no clear indication of further increase of tone. The other deficits were as reported earlier.

When the animal was removed from the cage for examination on May 16 , and held by the body with all extremities extended downward, he could move all of them. However, if he were placed in a walking position, the right extremities dragged as if he did not know where they were, suggesting a handicap in locomotion from the loss of positional sense. One finger had an abrasion where he had caught it on the cage. When laid on his normal side and then turned over, he responded by attempting to turn back. He had a loss of fine movements, most noticeable on the fingers of the right hand and the toes of the right foot. The right homonymous hemianopia noted earlier was, of course, permanent.

The monkey improved very rapidly and, by May 18, could feed himself quite well and could stand and even walk a little by leaning against the wall. By May 23, his walking had improved and he was learning to climb the walls of the cage. By May 27, he had become quite active and could walk and climb quite rapidly. Because of a tendency to circle toward the side of the lesion due to the frontal lobe destruction, he preferred to walk along the wall with his left side toward it. It was felt that he used the wall not only for support but to prevent circling. In walking, he stretched out his right paretic upper extremity and placed it very carefully, often looking at it, and he dragged his right lower extremity slightly. The same increased tonus persisted. There was an obvious loss of fine movements of the fingers and the toes.

The animal was watched carefully during the summer and examined frequently. The report on October 6, 1959, approximately 5 months after the operation, states that the monkey could climb around the cage quite easily. When not using his right arm he held it in the typical hemiparetic position. He had obviously lost fine movements of the fingers and the toes. When taken out of the cage for examination, the fingers were flexed with the thumb turned in, on the right. The right arm was slightly hypertonic as compared with the left. The right foot with its extended toes had the appearance characteristic of a hemiplegic foot and the right leg was slightly extended. However, only a minimal increase of tonus was demonstrable in the right leg when compared with that found in the left leg.

Among other animals with subtotal unilateral cortectomies (some with and some without basal ganglion involvements) were Monkeys 772, 874 (fig. 5e,f), 3998 (fig. 5a), 4109, 4981a, 5028 (fig. $5 \mathrm{c}, \mathrm{d}$ ), 5316 and 5337 (fig. 5b). The results obtained from observations of these animals were confirmatory of those described, differing only in minor detajls. Their case histories are available but space does not permit their presentation in this paper.

On February 4, 1955, a left craniectomy was performed and the dura retracted on Monkey 2007. Both the cortex and the basal ganglia were removed on the left. Fluid was given by intravenous injection during the operation. In spite of this, postoperatively the animal showed considerable shock.

By February 6 , the monkey had begun to recover and, at that time, showed a hemiparesis on the side opposite the lesion, evidenced particularly by the paretic positions of the hand and the foot and the loss of highly specialized movements. There was a lack of placing on the right and a right homonymous hemianopia. At this time, the animal was unable to stand.

During the succeeding two weeks, although the motor and the sensory deficits present on February 6 remained, the animal gradually regained an upright position and, by the end of the period, could walk quite well. He was decidedly hyperkinetic and moved restlessly backward and forward in the cage in the stereotyped manner of a monkey with a large frontal lobe lesion.

Attempts to take movies of this animal, on March 14, were unsuccessful since, as soon as the lights were turned on, he crouched down on the bottom of the cage. At other times he exhibited whirling movements and a tendency to jump toward the side of the lesion. On the slightest stimulation, he showed rage responses, baring 

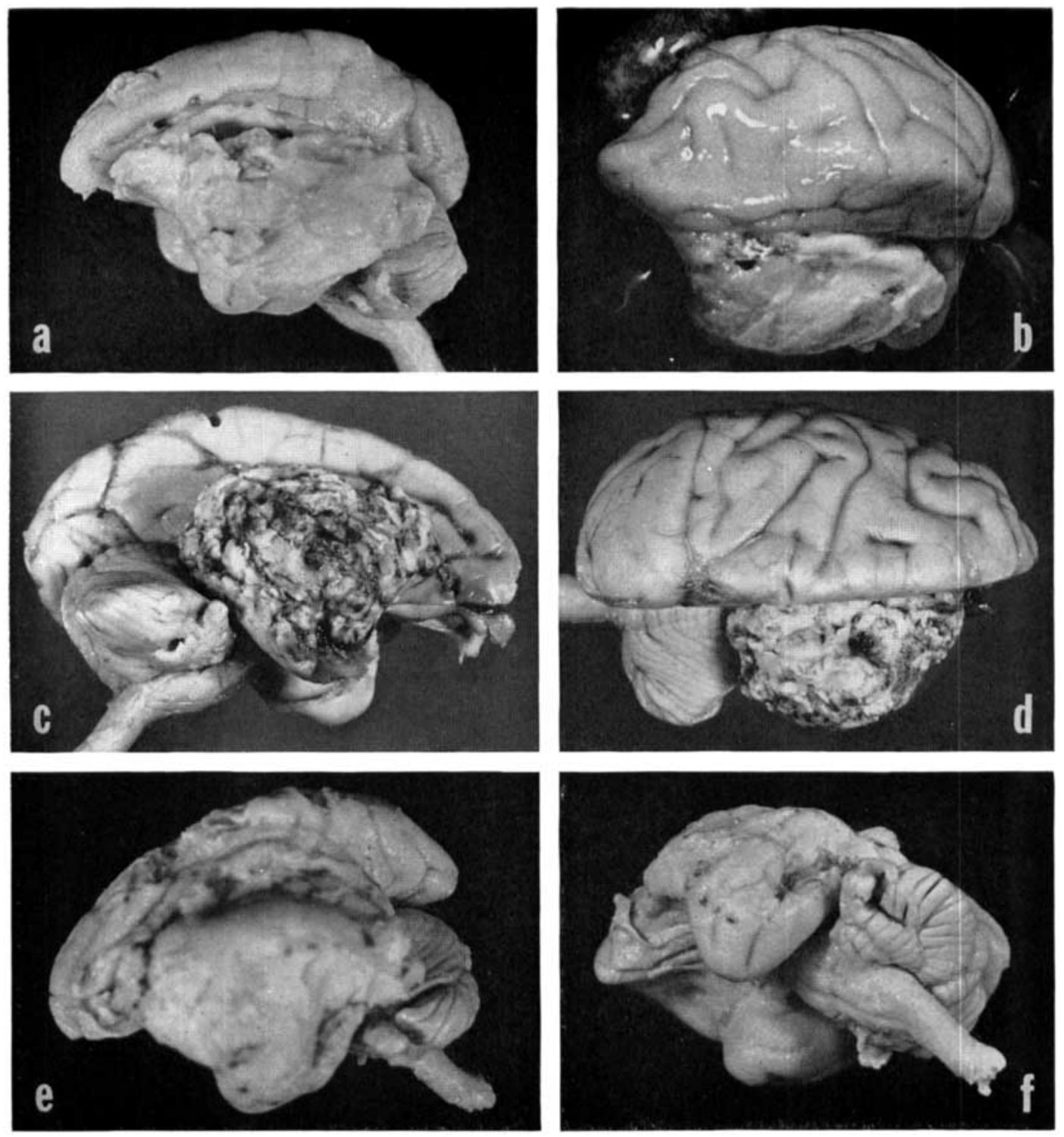

Fig. 5 Postmortem views of the brains from Monkeys (a) 3998, (b) 5337, (c-d) 5028, (e-f) 874 .

his teeth and grabbing the floor of the cage and shaking it vigorously. He had piloerection particularly around the shoulders. There was some increase in tone in his right arm but no true spasticity. It was difficult to determine to what extent the increased tonus was due to the animal's unwillingness to cooperate. He often stood on three legs with the right foot not touching the floor. Possibly this was an indication of his loss of positional sense. When not walking, he kept his right arm flexed without movement of hand or forearm. He acted as if he did not know the right side existed, even though he could walk.
On March 24, the animal showed a clasp-knife rigidity and a dorsiflexion of the right ankle. The right arm was held in a hemiplegic position. Considerable movement of the right arm and leg were possible on the side opposite the lesion, but fine movements of fingers and toes were lacking on that side. The circling movements toward the left and the jumping toward the left when excited persisted and the animal still appeared hyperkinetic and also highly enraged. On September 29,1955 , the right cerebellar hemisphere was excised. 
On July 26, 1960, a left cortectomy was performed on Monkey 4997, with slight invasion of the head of the caudate but with the remainder of the basal ganglia and the thalamus uninjured. On the day following the operation, the animal had a pronounced head deviation (which persisted for several days) and a hemiparesis on the side opposite the lesion. There was no increased tonus.

By July 28, the monkey could pull himself around the cage with his left arm, could stand if supported by the cage wall, and was beginning to feed himself. When tested on August 1, he showed a right homonymous hemianopia, an inability to place on the right, possibly some evidences of a loss of discriminatory tactile sensibility, particularly on his hand (indicated by his better recognition of an object, such as a grape when it was placed in his left rather than his right hand and he was not permitted to look at it), and a mild hypotonicity with a loss of fine movements on the right. Strength appeared to be somewhat better in the upper extremity than in the lower. The circling to the left, due to the frontal lobe involvement, still remained, but the turning of the head was no longer evident. The animal was in excellent physical condition and was already trying to walk by leaning against the wall. During the succeeding days, he learned to walk and climb very well. Except for the loss of individual movements of the fingers and toes, the motor weakness was not apparent. The Ioss of positional sense was marked and was probably his greatest handicap. The right homonymous hemianopia remained. The tone was approximately normal. The animal was sacrificed on September 22, 1962.

Another monkey (Monkey 571), in which the cortex on the left hemisphere was removed and the caudate nucleus injured, was sluggish and sleepy, but moved all extremities on the day following operation (September 5, 1958). She was not able to stand or to move around the cage.

On September 6, she began pulling herself around the cage and when, on September 8 , she was removed from the cage for better examination, it was found that she could stand erect and could walk if she leaned against the wall for support. As usual, such support was needed on the side of the body on which the lesion had been made, that is, on the side opposite the hemiparesis. Testing showed a loss of placing of the involved upper and lower extremities and that fact, together with the way the animal dragged her foot, suggested a loss of positional sense on the side opposite the lesion. The tests for loss of discriminatory tactile sense were not satisfactory. There was an evident loss of individual finger and toe movements on the side of the hemiplegia, a slight head deviation due to a caudate involvement, and a right homonymous hemianopia. There was a tendency to hold the right arm in a somewhat flexed position at the elbow when the animal was at rest, but no true increase in tonus was demonstrable. The head deviation disappeared before the end of the month, but the other losses persisted. When moving pictures were taken on October 16, the tendency of the animal to circle due to the frontal lobe involvement was

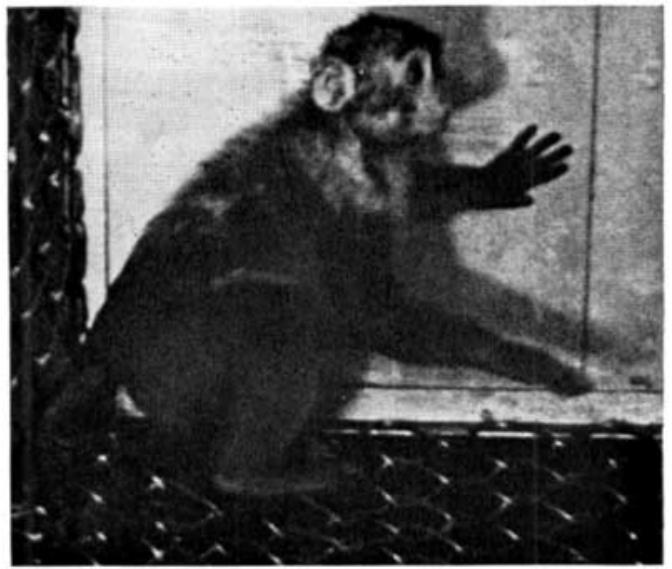

Fig. 6 Monkey 571, November 11, 1958, following left hemispherectomy.

very evident although she could walk quite well. Her arm was held in a somewhat flexed position at the elbow with the fingers outstretched (fig. 6). Her slight awkwardness, possibly an apraxia, was revealed as she moved around the cage. During the next month there was little change. However, pictures taken during November showed the posturing of the right hand (fig. 6) and the right hindleg. At no time had this animal shown an increase in tonus. On November 13, 1958, the monkey underwent a further operative procedure.

\section{Hemicortectomies in children}

Total or subtotal hemicortectomies involving the non-dominant hemisphere in man produce many of the same signs seen in the monkey. There is a contralateral homonymous hemianopia, a loss of positional sense and of highly discriminatory tactile sensibility in man (it is hard to prove in the monkey) and an astereognosis on the side opposite to the lesion. Sometimes apraxias or other signs of involvement of the associational fields may appear. There are fewer changes of associational type in a child in which a hemisphere lesion has developed earlier than in an adult who had a relatively normal cortex bilaterally for many years. Presumably, the child never built any (or many) associational patterns in the injured cortical area and so lost little when it was removed.

On the motor side, there will be a permanent loss of fine movements (such as individual finger movements), a central paresis of the lower part of the face, the tongue, and the uvula on the side opposite the lesion and often a transient deviation of 
the eyes toward the side of the lesion. The child, like the monkey, may walk again and carry on a fairly normal life. He may even be better adjusted to his surroundings than preoperatively. This is not meant to imply that the intellectual potential of any normal individual will not be significantly lessened by a hemicortectomy or a hemispherectomy. Removal of the cortex from the dominant hemisphere is avoided if possible.

LAUREN had had a stormy birth history, having been the product of a rather prolonged and difficult labor. At nine months she was diagnosed as a cerebral palsy child but she did not have her first convulsive seizure, which involved the left arm and the left leg, until she was eight years old. She continued to have seizures intermittently for some time but they gradually became worse until they were very severe and of two types. One type was a left Jacksonian seizure; the other was of psychomotor or temporal lobe type with associated déjà vu phenomena and macropsia. A neurosurgeon, Dr. Francis Martin, performed a pneumoencephalogram and demonstrated a marked cerebral atrophy and dilation of the lateral ventricle on the right. Every attempt was made to control this child's seizures by anticonvulsant medication but all attempts failed. She was then referred to the neurosurgical group at the University of Michigan Medical Center for a right hemispherectomy.

At the time of her admission to the University of Michigan Medical Center Lauren was 15 years old. She had a childish type of behavior. She had bursts of laughter, a marked interest in the opposite sex, and a rather bizarre type of behavior pattern.

Injection of the carotid arteries, bilaterally, demonstrated a thrombosed or undeveloped right middle cerebral artery. Both of the anterior cerebral arteries filled from a right-sided injection. An electroencephalogram showed that the entire right hemisphere had a marked delta wave pattern.

A right frontotemporoparietal craniectomy was performed on November 30, 1960 and a huge porencephalic lesion, following the distribution of the middle cerebral artery, was disclosed (fig. 7). Electrocortigraphic recordings taken during the operation showed a hemispheric abnormality on the right. An almost complete right hemispherectomy was performed with an attempt to spare

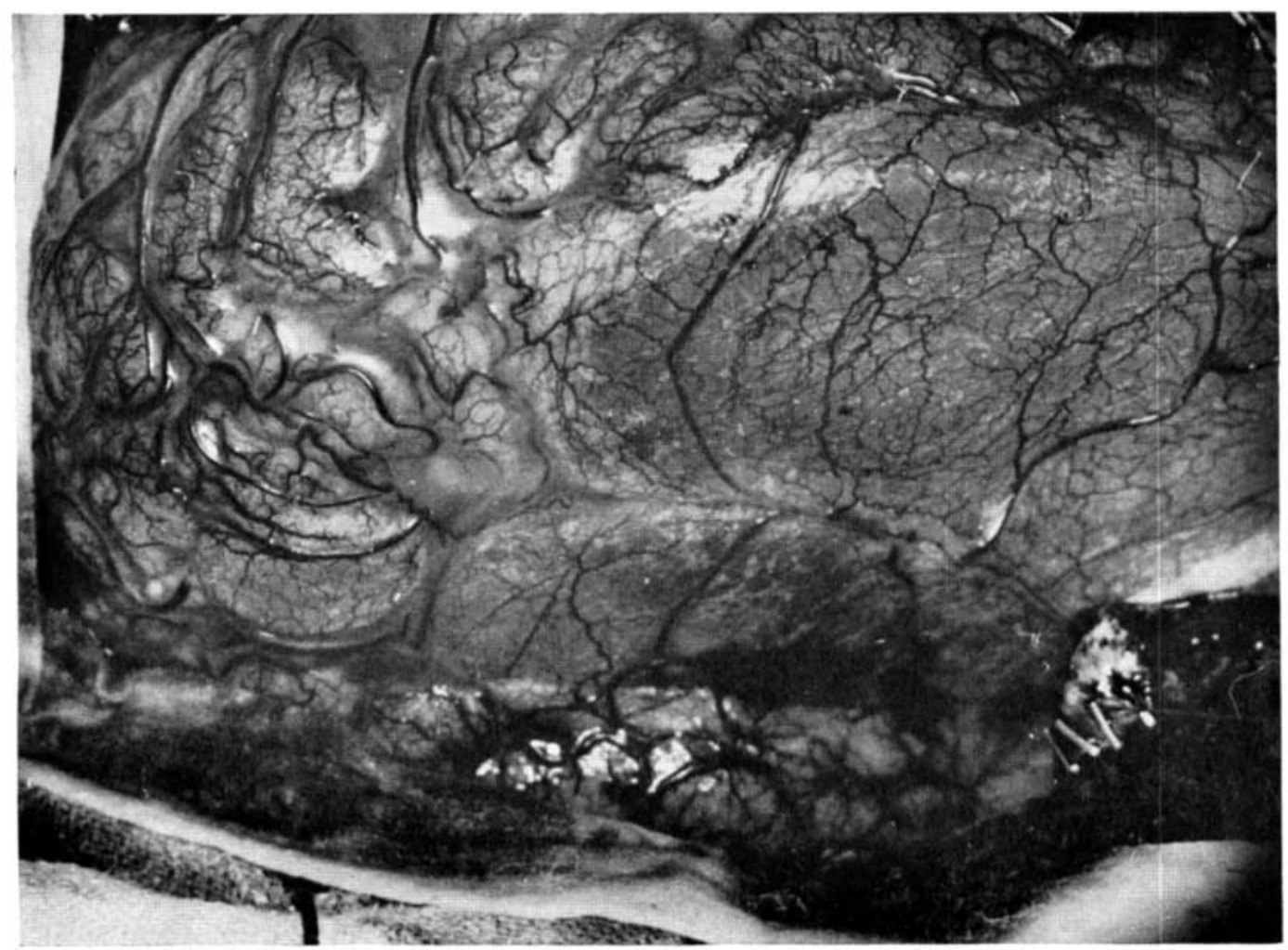

Fig. 7 Porencephalic cyst of right frontotemporoparietal region.

This figure, and figure 8, are reproduced from the Journal of the Michigan State Medical Society, 62: 175-204, Schneider and Crosby ('63), by permission of the publishers. 
the anterior cerebral artery in order to provide collateral blood supply to the left hemisphere. Postoperatively, the child did well for 12 hours. Then she had an increase in blood pressure, diminished respiration, and dilated fixed pupils. She was returned to the operating room and a large clot was found in the space where the right hemisphere had been removed. The tentorium was cut and the wound closed. The child made an uneventful recovery.

Postoperatively, this child has remained seizure free. She has less spasticity in her left upper extremity than before operation (fig. 8). In general, her mental development and her behavior are better than preoperatively. Her school work has gradually improved and she graduated from high school in June, 1964.

\section{Bilateral cortectomies in monkeys}

Total and subtotal bicortectomies, some with and some without basal ganglion in-

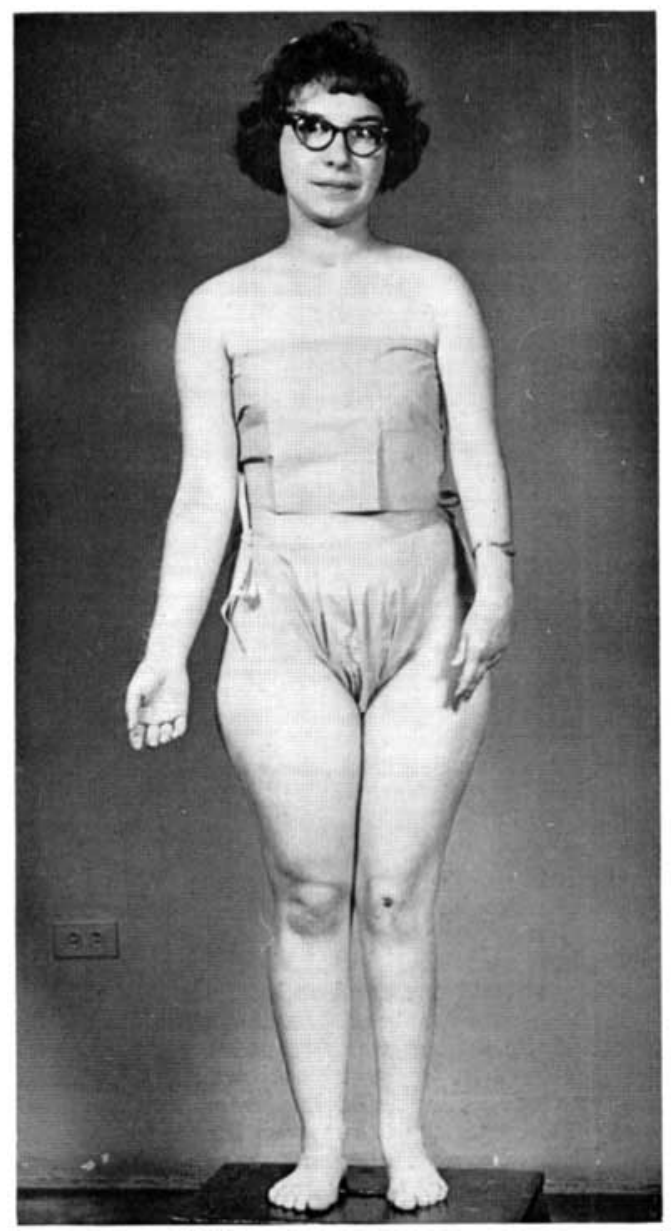

Fig. 8 Lauren two years postoperatively. For acknowledgment, see figure 7 . volvement, were attempted and seven animals survived. All of these bicortectomies were the final results of additive lesions, for, in the series here reported, the cortex was not removed on both sides at a single operation. Sometimes a series of operations was employed in producing a bicorticate animal. All seven animals which had undergone bilateral cortectomies (Monkeys $571,732,4755,4806,4865,4981$, and 5398) showed certain common deficits and certain typical reactions.

Five of the animals in this group had apparently complete cortical blindness (even though there was a little sparing of the inferior occipital region on the right in Monkey 4806). Monkey 571 had some useful vision on the left due to a deliberate sparing of the right occipital cortex and part, at least, of the radiations to it. In these monkeys, that were otherwise blind, a slow blinking sometimes occurred when the hand was thrust suddenly toward the animal's face, but the experimenters were never quite certain that this was due to visual stimulation (that is, ares which relayed through the superior colliculus and never reached the cortex). It is conceivable that it was brought about by stimulation of the face from the air currents set up by the moving object. All of the monkeys, of course, had light reflexes which do not depend on arcs through the cortex.

Even when the animals were not too stiff to move their limbs, no placing could be elicited and there is little question that all types of discriminatory somesthetic responses were lacking. Taste and smell were not checked. The animals jerked when sudden loud noises were made, such as clapping the hands or dropping a tin pan. That they recognized tones of different pitch is probable (though not proven here) in view of the experimental work of Diamond and Neff ('57) and others, but there was no evidence that the sounds the monkeys heard were very meaningful. The animals responded to touching and sometimes to painful stimuli by jerky movements.

Precise, fine movements of single fingers or toes were lacking bilaterally. The animal with a total or subtotal bicortectomy did not walk, stand erect, feed or care for himself, or carry on any purposeful activ- 
ity. Unless very ill, he slept and awakened approximately the normal amount. He was not in coma. He even had some sort of dim awareness when he was moved about and sometimes cried, but he had no interest in his surroundings unless actively stimulated. He displayed no ambition or intelligence. Consequently, even when the hypertonicity was reduced by removal of some of the cerebellum and he might conceivably have walked again, and certainly fed himself, he showed no desire to do anything at all. He had reached the point of no return.

Such bicorticate animals had a loss of precise, fine movements most marked on the extremities and a similar loss of cortical automatic associated movements so that they often had very expressionless faces. On stimulation, such animals gave jerky responses and, if the tonus were not too great, moved all extremities in what certainly appeared to be progression movements. Finally, the tonus which followed the first cortectomy was approximately normal after the animal had stabilized, providing the basal ganglia had not been greatly involved. In those animals in which there had been considerable involvement of the basal ganglia, there was evidence of increased hypertonicity, although it was not very incapacitating in amount and was most evident when the animal was at rest (p. 13). In the bilateral cortectomies this was not true. Whether there were some bilateral (Monkey 4806) or limited unilateral (Monkeys 571, 4865) basal ganglion lesions, or no basal ganglion involvement (Monkeys 4755, 5398), there was a gradual development of hypertonicity, which ultimately became an opisthotonos if the monkey survived long enough.

On November 13, 1958, Monkey 571, that had survived (p. 13) with the usual deficits, a leftsided removal of the cerebral cortex and portions of the basal ganglia, was reoperated and a subtotal removal of the right cerebral cortex was carried out with sparing of the occipital and some of the adjoining portions of the posterior temporal and the posterior parietal cortices. Postoperatively, the animal gave jerky movements when touched.

Because of her weakened condition, she was not fully tested until four days after her operation. At that time, her right arm and, to a slightly less degree, her right leg, were extended and rigid, with the right foot fanned and the toes extended. The extremities on the left were slightly flaccid. The left upper extremity was flexed but there was some ability to move the fingers. The head was bent backward in opisthotonos, the pupils were equal and reacted to light, and the eyes deviated to the right (fig. 9). Pinching of the trunk or of either leg resulted in a flexion of the left arm and the right leg and a flipping movement of the right leg toward the left. These movements were followed by a clonic Jacksonian seizure in which the right arm extended and revolved around the shoulder, the right leg extended, and the eyes turned up (fig. 10). In the postictal stage, the right leg relaxed.

By December 1, there was increased tonus on the left as well as on the right, but not so severe. When the animal was stimulated, the head raised slightly and the eyes jerked upward toward the left. Both right extremities were rigid, with the lower one extended and the upper one adducted and held posteriorly. In an attempt to relieve the tonus, a suboccipital craniotomy was carried out, the dura reflected, and an attempt made to remove part of the cerebellum. The animal succumbed.

Postmortem examination showed that nearly all of the cortex had been removed from the left hemisphere of Monkey 571 except for small remnants in the region of the lamina terminalis and in the temporal lobe, where a small part of the amygdala had been spared. The putamen had been destroyed and there was an invasion of the globus pallidus, the dorsal thalamus, and the midbrain. There was considerable injury to the head of the caudate. On the right, (fig. 11) the cortex had been removed except for the intentional sparing of the occipital lobe and the more posterior temporal and parietal areas (fig. 12).

The early experimental data on Monkey 4755 has already been reported (p. 10). On May 5, 1959 a second and a right cortectomy was carried out. Examination, on May 6, showed that the upper part of the animal's body and head were tilted toward the left, with the chin turned toward the right. There was increased extensor tonus in both lower extremities, but, although the right leg was rigidly extended, the left leg could be moved at ankle, knee, and hip. Both upper extremities could be moved at all joints and marked grasp reflexes could be elicited except from the very rigid right foot. An action tremor was present in the upper extremities. The eyes had to-and-fro movements, the eyelids showed fluttering movements, and the left eye was open. The animal was blind but had light reflexes.

As the animal lay on her left side on May 8, the right arm was flexed at the elbow and the wrist and the fingers were extended. Some movement occurred in this extremity when the animal was touched. The right leg was flexed at all joints. The extensor tonus was increased on the left. When the animal was turned on her right side, the position of the extremities remained the same except that the right leg was extended. Strong grasp reflexes were elicitable from all extremities. 

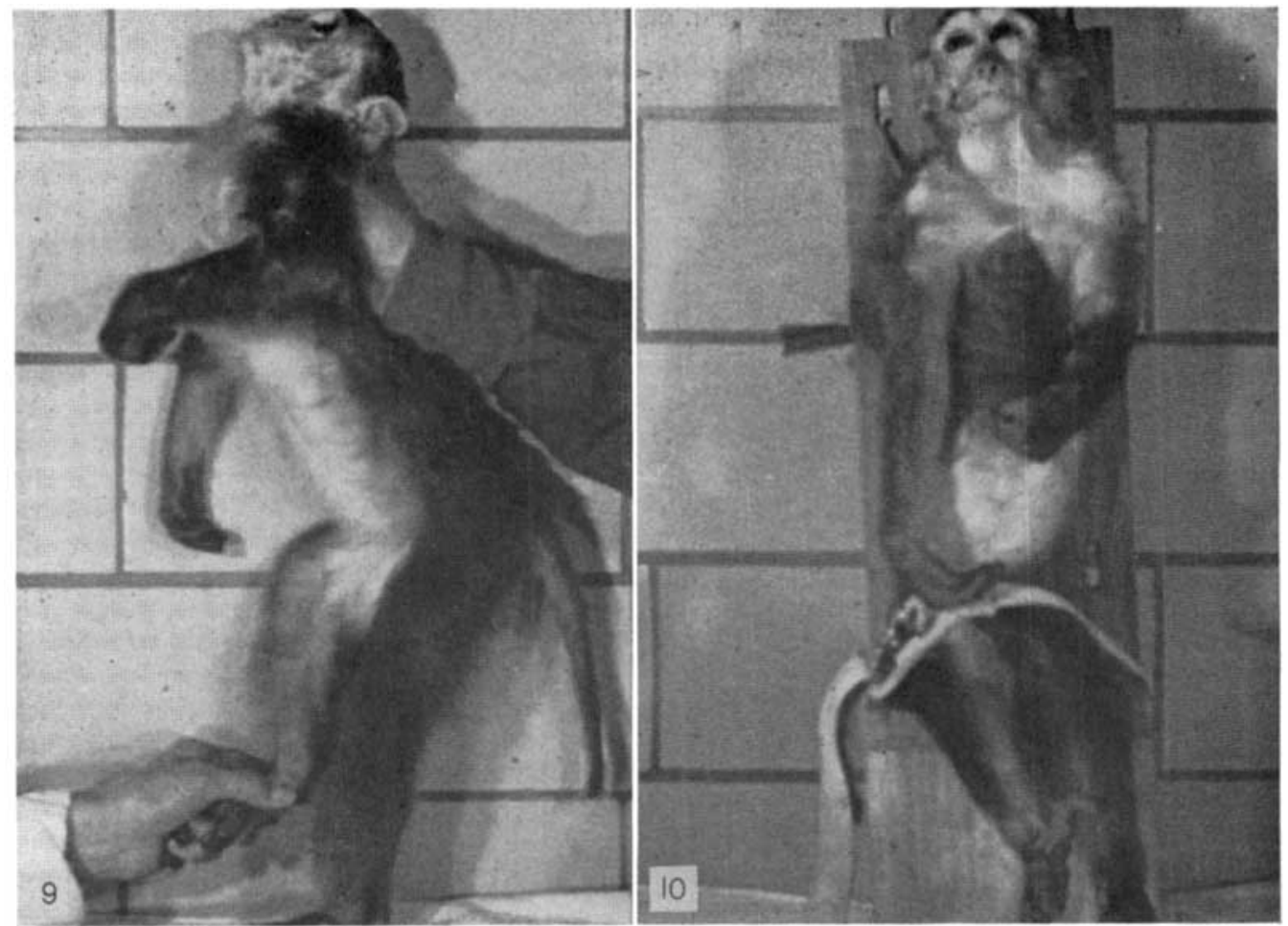

Fig. 9 Monkey 571, November 18, 1958, following an incomplete bilateral cortectomy. Fig. 10 Monkey 571 during a seizure.

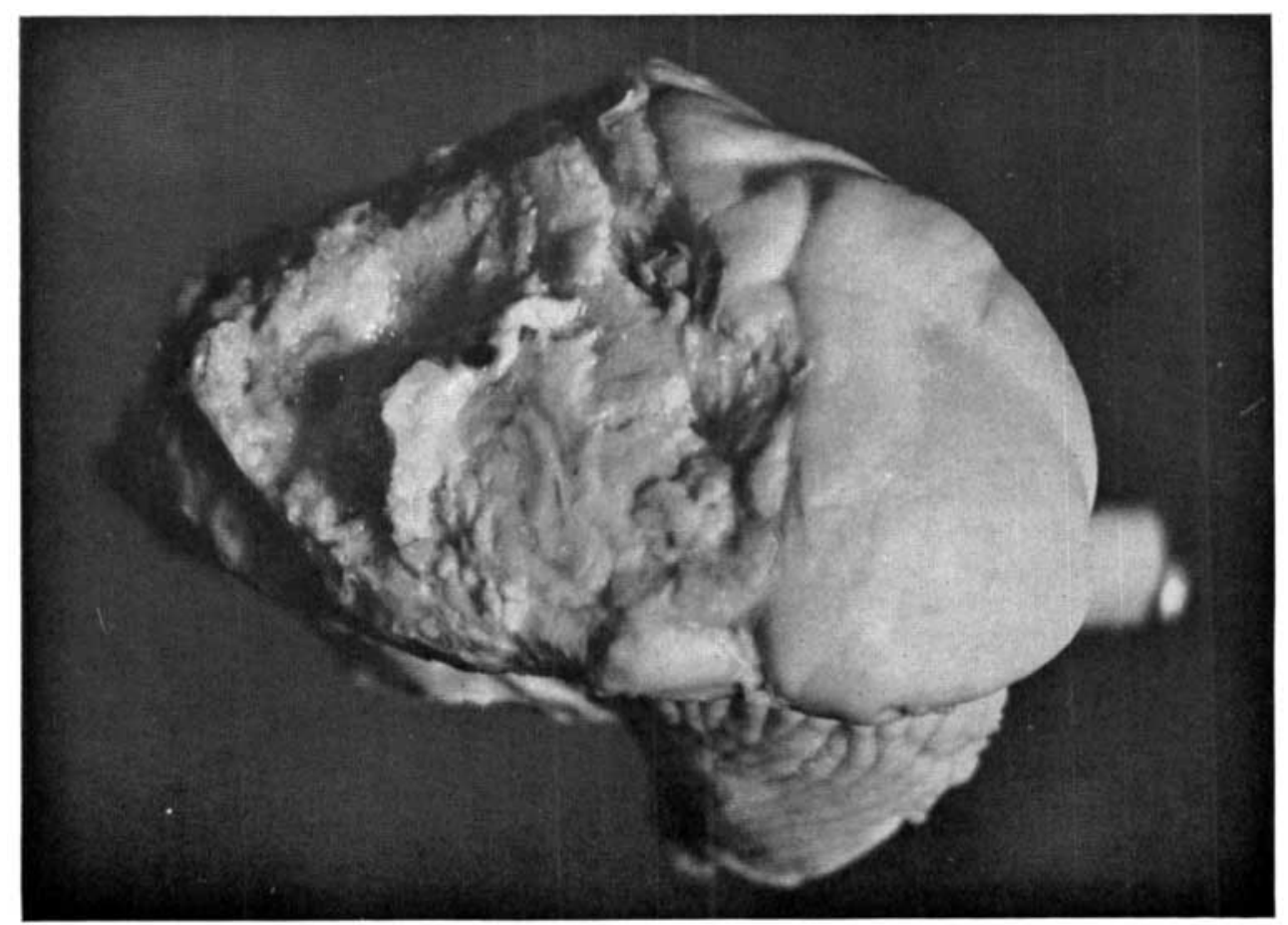

Fig. 11 Brain of Monkey 571 showing incomplete bilateral cortectomy. 


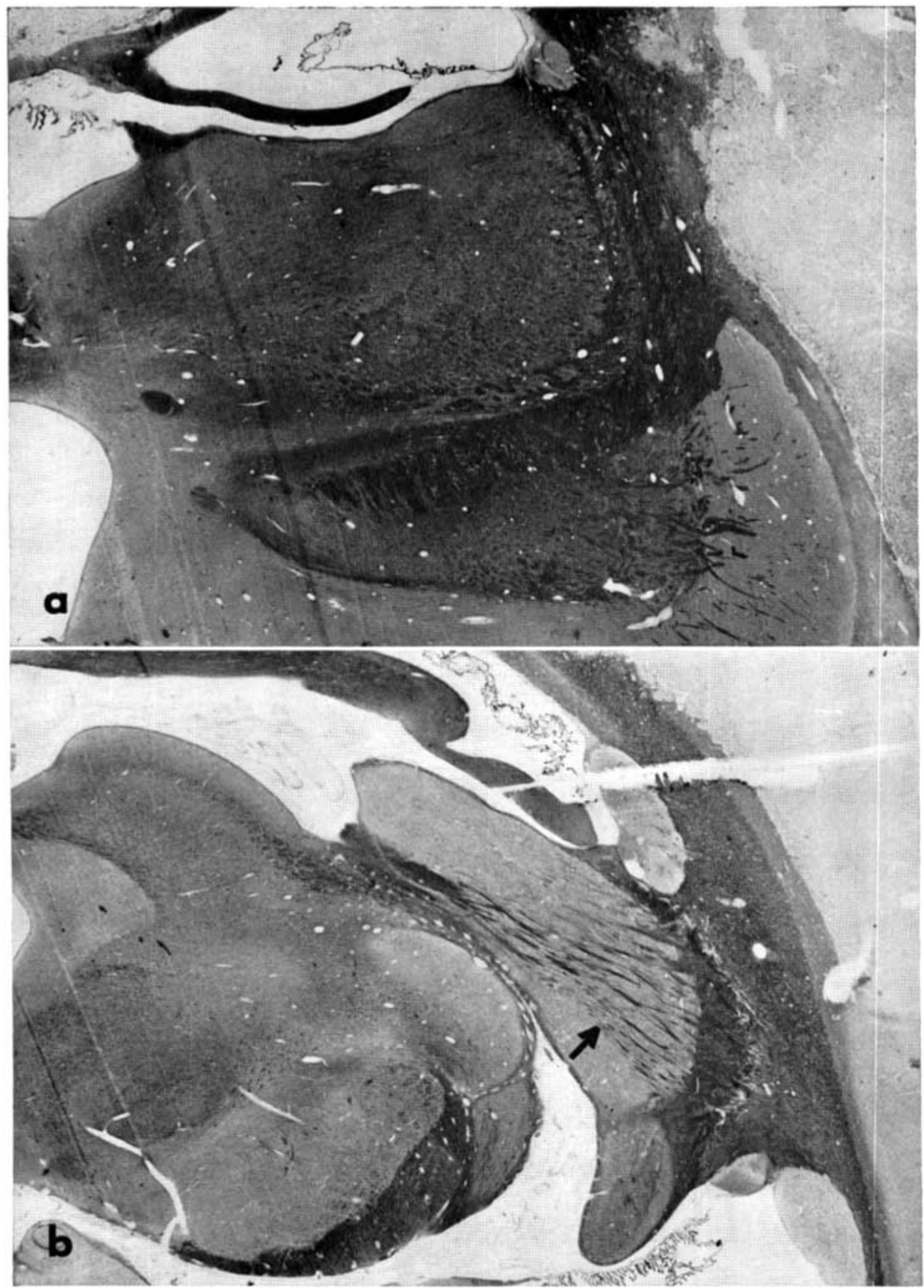

Fig. $12 \mathrm{a}-\mathrm{b}$ Brain of Monkey 571. (a) Section through the right lateral and ventral nuclei of the dorsal thalamus to show the sparing of the basal ganglia and thalamus, $6 \times$. (b) Section through the rostral midbrain and caudal end of the pulvinar showing the spared corticotectal fibers (arrow), $6 \times$. 


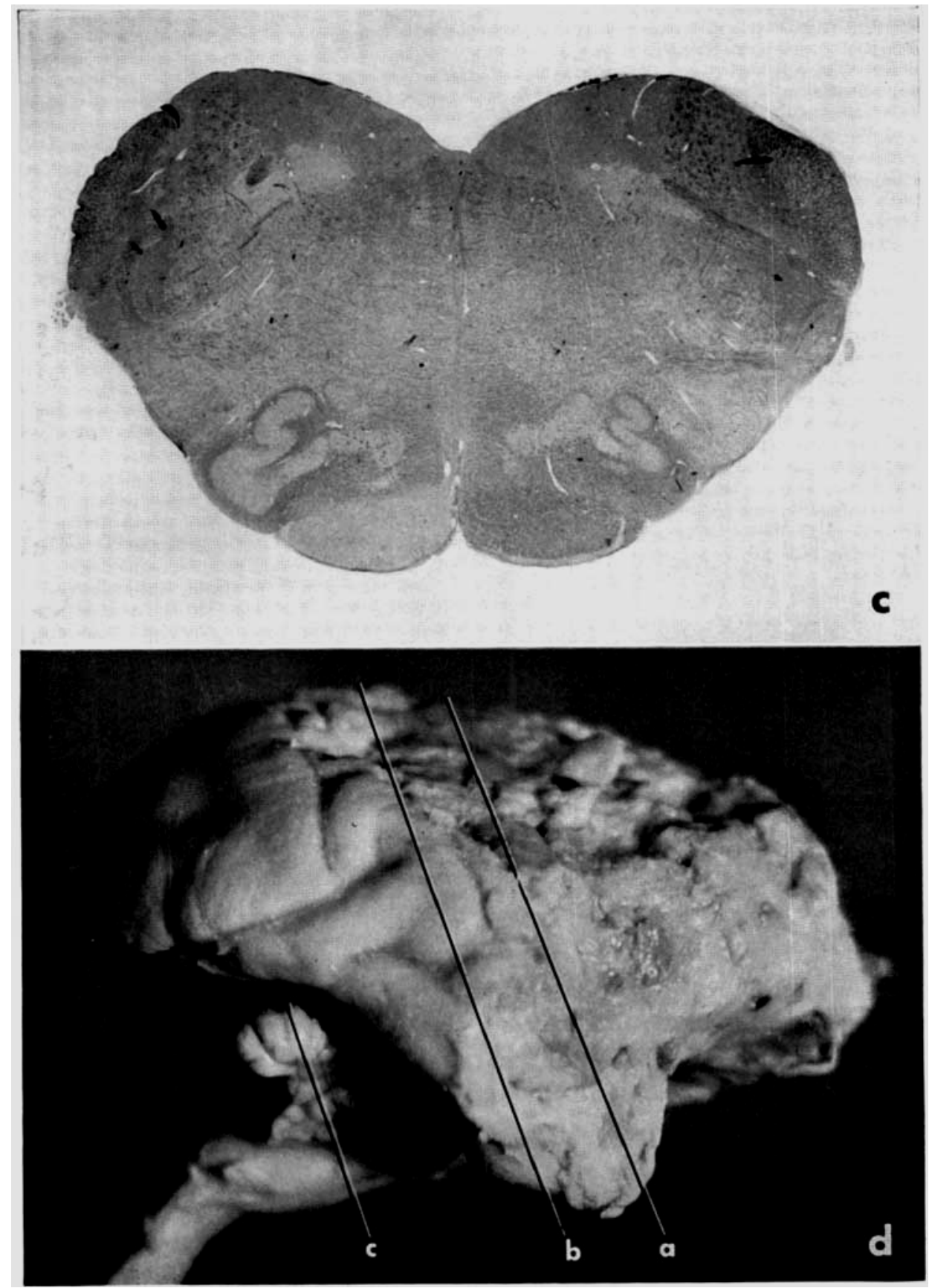

Fig. $12 \mathrm{c}-\mathrm{d}$ (c) Section through the medulla at the level of the vagal and hypoglossal nuclei. On the left note the marked degeneration of the pyramidal tract and on the right only some hint of early degeneration, $9 \times$. (d) Planes of sections $a, b$, and $c$. 
During the succeeding days, there was increased tonus on the left and some clenching of the right fist. The animal made no attempt to eat, but would swallow food placed in her mouth. She developed a violent tremor on being moved or fed. She had no interest in anything and had only a slight grade of awareness, yet she was not in coma and not asleep. She was provided with a foam rubber mattress and, finally, a hammock when she developed a pressure sore. The arms were bandaged in splints to try to prevent contractures. She was given baths and actually had the services of a technician who had been an army nurse. By May 15, her rigidity was extreme although she did try to move a little and learned to suck from a bottle (fig. 13). On June 18, 1959, portions of the cerebellum were removed.

Monkey 4806 (p. 5), having the usual effects of a right hemicortectomy (with evidence of a slight basal ganglion involvement), had the cerebral cortex removed on the left on December 9, 1958. By the next day she showed the beginning of hypertonicity and marked motor deficits. She could not stand and did not move around in the cage. On December 11, there was marked rigidity on the left, slightly greater in the leg than in the arm. On the right, the tonus was approximately normal and on this side, a grasp reflex (fig. 14) could be demonstrated. The bilateral loss of fine movements and the sensory defects, including blindness, were as to be expected. Light reflexes were present. The animal developed Jack-

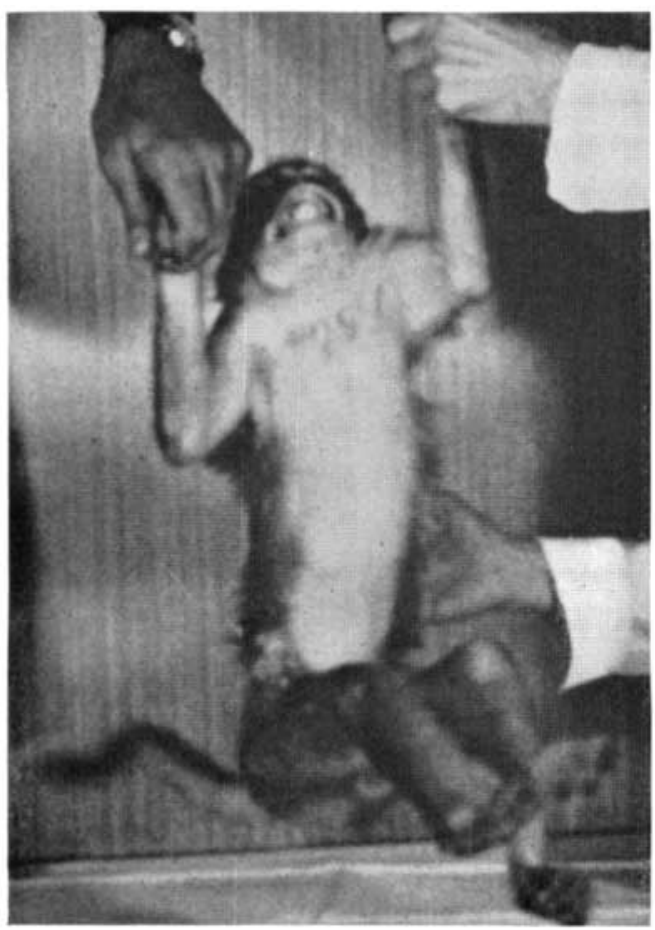

Fig. 13 Monkey 4755 on June 9, 1959.

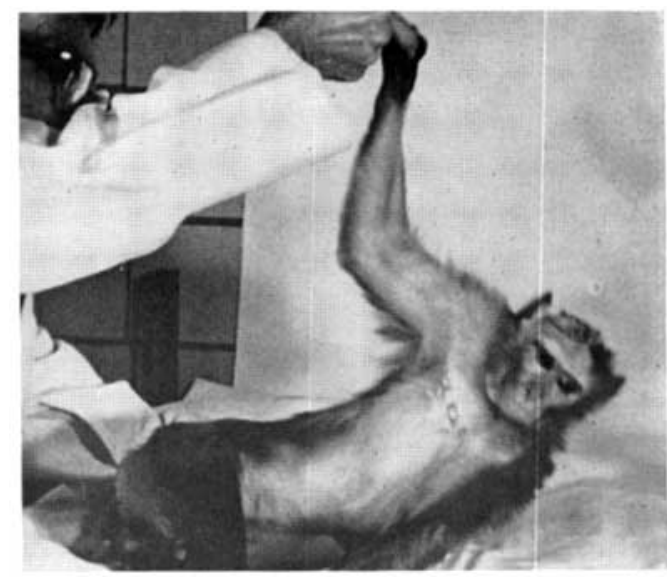

Fig. 14 Monkey 4806, December 23, 1958, with pronounced grasp reflex after bilateral cortectomy.

sonian convulsions in which the lower extremities moved alternately and the arm on the side of the second lesion grasped the monkey's head. The right arm either did not move or grasped her right foot.

On December 16, 1958, the monkey showed marked hypertonicity although the tonus was perhaps slightly less on the right than on the left. On December 23, there was not much change. The lower extremities and the left arm showed marked rigidity and the hand was bent at the metacarpal-phalangeal joints. The right arm was flexed with the fist clenched. The monkey had a masked face (fig. 15). There was an intention tremor. The hair was erected over the upper part of the body, but there was a decrease in the early irritability and some lethargy. The animal was sacrificed and the brain photographed on January 4, 1959 .

Postmortem examination of the brain of Monkey 4806 showed that, on the right side, there had been some preservation of a small inferior portion of the occipital cortex and of the adjoining temporal cortex and that the lenticular nucleus had been slightly injured. On the left, the entire cortex, the putamen, and the outer part of the globus pallidus had been removed.

On August 8, 1961, Monkey 5398 had had a left hemicortectomy and, 21 days later, showed the usual effects of such a procedure after an animal had stabilized. He walked well, but had a loss of fine movements on the right, an inability to place, a loss of positional sense on that side, and a right homonymous hemianopia.

On August 29, a part of the right cerebral cortex was removed through a right craniectomy. On the following day, the animal lay on the bottom of his cage and showed some increased tonus but could move all extremities. Up to the time of his death, September 3 , from an acute massive brain hemorrhage from the anterior cerebral artery (fig. 16), a slight hypotonicity persisted on the side of the lesion. He moved the 


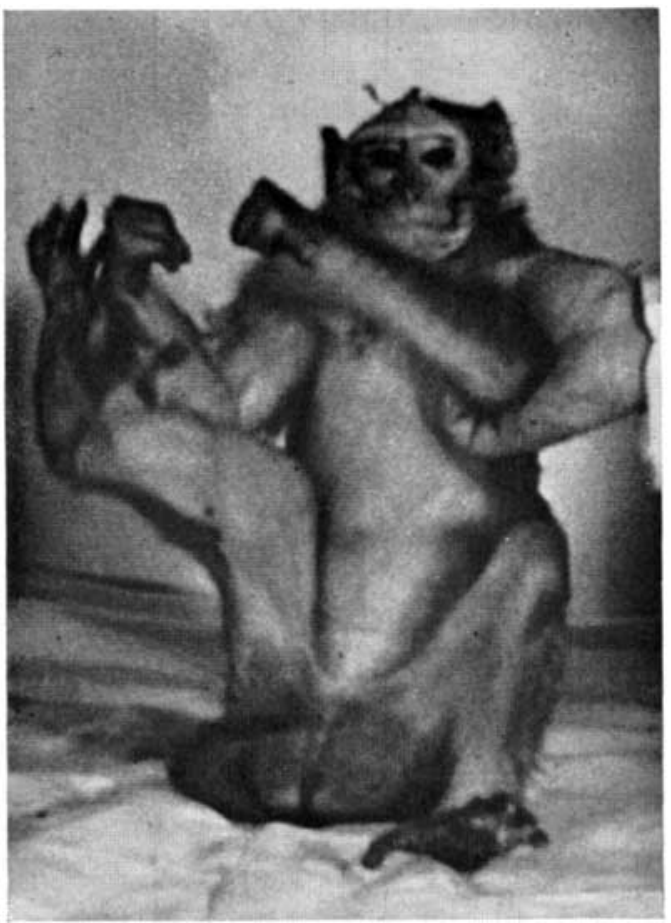

Fig. 15 Monkey 4806, December 23, 1958, one week following the second cortectomy.

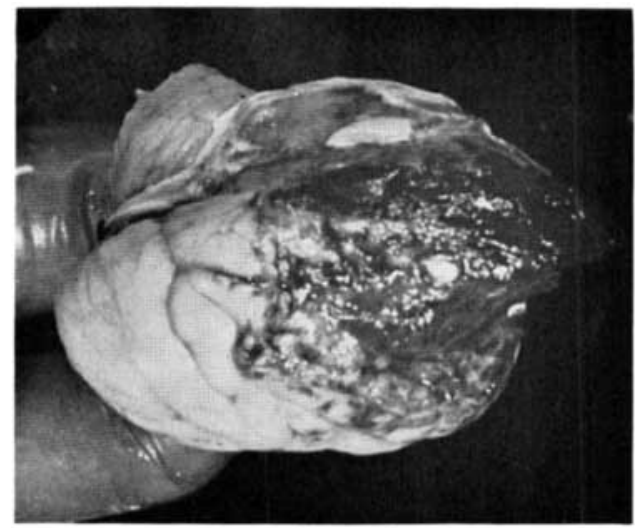

Fig, 16 Postmortem appearance of brain of Monkey 5398.

right side better than the left side, where marked hypertonicity was developing. The left hand was clenched and the left arm bent at wrist and elbow. He blinked when a hand was thrust suddenly before his eyes (whether as the result of visual or tactile stimulation) but otherwise gave no evidence of having cortical vision. Light reflexes were present.

Postmortem examination showed that Monkey 5398 had had a complete left cortectomy with a sparing of the basal ganglia and the thalamus. On the right, the front end of the cerebral cortex, the hippocampus and the amygdala had been removed. There was marked evidence of a large hemorrhage (fig. 16).

\section{Bilateral cortectomies in children}

Bilateral subtotal or total destruction of the cerebral cortex in man, as in the monkey, represents a condition from which there is no return and a loss for which there is no useful substitution. A child with such a devastating lesion resembles in many ways the monkeys with subtotal and total bicortications. The following case history illustrates these statements.

SUSAN was said to have been perfectly normal until 14 months of age at which time she developed an ear infection and had a temperature of $105^{\circ}$. During this illness she convulsed for a period of 13 hours, after which she had a residual left hemiparesis and a left internal strabismus. From that time, the child had convulsive seizures which were clonic and tonic. Sometimes she would lie down on the floor and just stare into space. These occurred 20 or 30 times a day in spite of careful anticonvulsive therapy.

When she was three years old, she was examined by the neurosurgery staff at the University of Michigan Medical Center. There was an equinus deformity of the left foot, with ankylosis of the left ankle. The legs were of equal length. There was a right pelvic tilt. The child was extremely uncooperative and irritable. She could not speak plainly and it was difficult to evaluate her mental status and intelligence. Except for the internal strabismus on the left, indicating a paralysis of the ipsilateral abducens nerve, her cranial nerves were normal. There was marked weakness of the left arm and the left leg. She had a circumduction type of gait and was quite unsteady. The deep reflexes on the left were hyperactive and the Babinski positive on that side.

The child's skull and chest films were normal Her pneumoencephalogram demonstrated a marked degree of right cerebral atrophy. Her electroencephalogram showed maximal involvement of the right hemisphere with occasional shifts of slower waves into the left hemisphere. Seizure formations were present in any two seconds of the patient's recording. The greatest spike bursts, however, were predominantly on the right side.

On July 19, 1954, the patient had a right frontoparietotemporal craniotomy with electrocorticography; a right hemispherectomy was performed. Postoperatively she convulsed, requiring pentothal for her seizures. Her temperature spiked to $108^{\circ}$. Following this, the child had coma and decerebrate rigidity. She was maintained on dilantin, phenobarbital, anticonvulsants, and gavage feeding. The fever persisted, averaging around $101^{\circ}$ daily with spikes to $103^{\circ}$. She developed severe opisthotonos and marked decerebrate rigidity (fig. 17). Repeated lumbar punctures were negative. She had a slight urinary tract infection. 


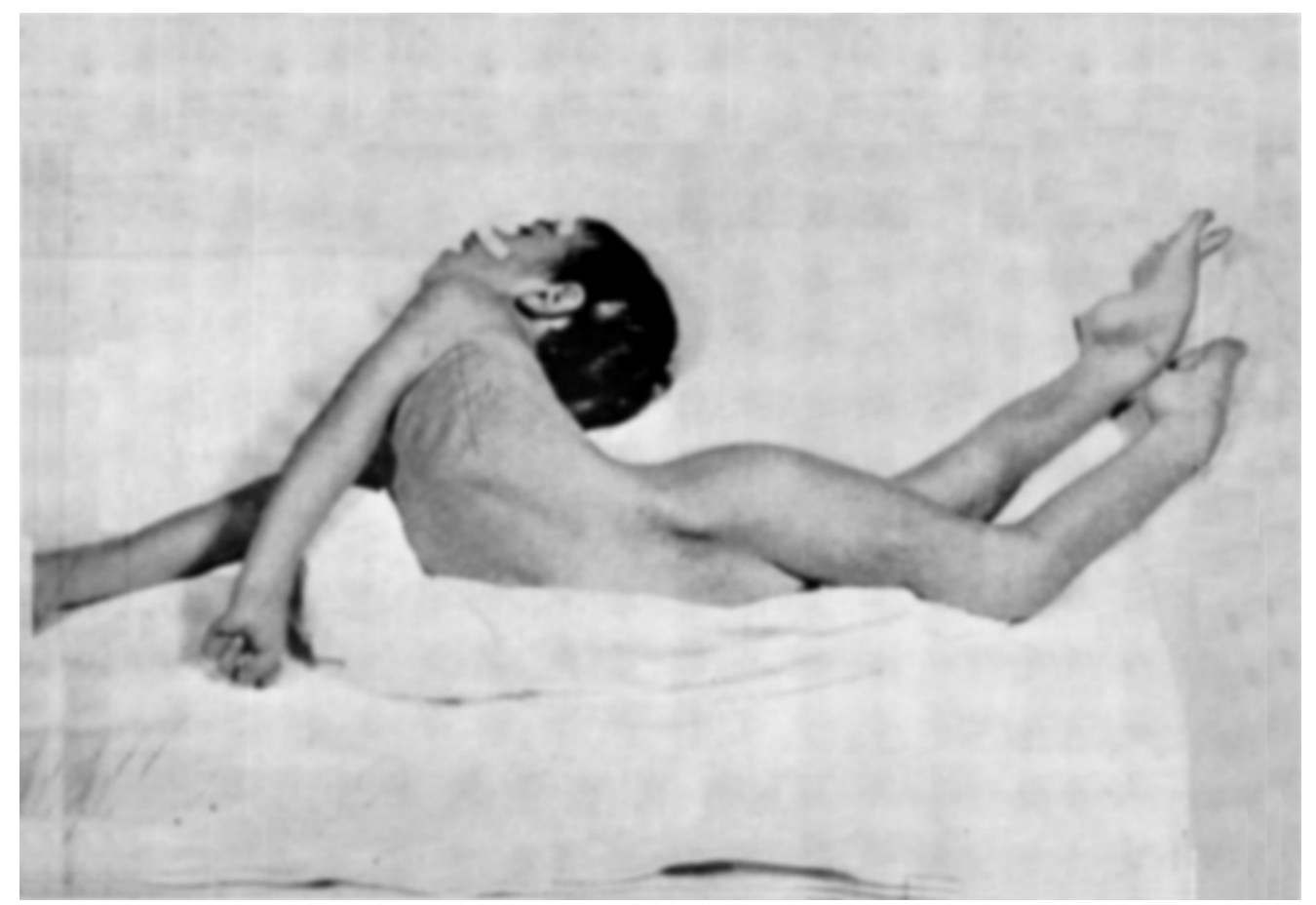

Fig. 17 Susan in the posture of decerebrate rigidity. (Reprinted from the Proceedings of the Second International Meeting of Neurobiologists, Amsterdam, 1959, by permission of the publishers, Elsevier Publishing Company - Schneider and Crosby, '60).

The patient cried in response to deep pain. Motor examination showed that the patient resisted flexion of the left elbow and extension of the wrists and the fingers bilaterally. In the lower extremities, full flexion of the thighs and knees occurred with resistance. No clonus was demonstrable. The reflexes were slightly hyperactive in the right upper extremity and the ankle jerks were absent in both lower extremities. There was a left extensor plantar reflex. There were no purposeless movements and no atrophy of the muscles.

The child succumbed at Caro State Hospital approximately five months after operation. She remained in a decerebrate condition until her death.

Post mortem examination disclosed the cortex on the unoperated side was thin and degenerated. The bilaterality of the cortical lesion was the cause of the opisthotonos.

\section{Lesions in cerebellum}

The cerebellar lesions selected as the basis for the present account differed widely as to size, depth, and location. They varied from a small lesion in the vermis or one hemisphere to such major involvements as subtotal or total cerebellectomies. Concomitant with the size and the location of a particular lesion, the effects varied from no observable permanent loss to major incapacitation following widespread cerebellar destruction.

\section{Lesions in vermis}

Stimulation of points on the pyramis or the uvula may bring about eye deviations (Cranmer, '51; Crosby, '53a, b; and others; monkeys of the present series). A small lesion in the vermal part of the posterior Iobe apparently produces no overt signs. Large lesions, which more or less completely destroy the posterior vermis (often including both the vermal cortex and the underlying fastigial nuclei), have very definite effects. After such an ablation, the animal is hypotonic and has a crouched wide-based gait, the spread of the hindlimbs being more marked than that of the forelimbs. If only the posterior vermis (including the nodule) is involved, the monkey's head tilts backward. This tilting of the head may be compared with the tendency of a patient with a similar lesion 
to fall backward. There may, or may not, be a nystagmus, depending upon whether the lesion in the vermal cortex and the underlying fastigial nuclei is equal bilaterally. If unequal on the two sides, a cerebellar type of nystagmus may appear. Such a nystagmus may disappear later if the lesion increases to involve both fastigial nuclei equally.

On February 17, 1960, a small lesion was placed in the left part of the pyramis (involving the cortex and the immediately underlying white matter) in Monkey 5251. The lesion extended slightly into the left paravermal area. The monkey showed no demonstrable defects from the time he recovered from the anesthetic until he was used for further experimental procedures on August 17, 1960.

On March 7, 1957, following a suboccipital craniectomy, a previously unoperated Monkey 4021 had the entire vermis removed. He recovered from the operation very well but, when examined the following day, showed a hypotonia and an ataxia. He had a crouched gait when he walked and a wide base when he attempted to stand During the succeeding days, he assumed only a slightly more erect posture. On March 9, the crouching gait and the wide base were still very evident and the hypotonia could be demonstrated easily. Neverthless, the animal managed to get around quite well.

On April 27, 1957, the animal stood with the forelimbs and hindlimbs widely spaced. When he moved around the cage, it was evident that he was having more difficulty in raising his hindlegs than his forelegs. With the support of the cage, he could get up on his hindlegs for a short time in order to pull himself up against the meshes. He had no paresis and no tremor.

On August 17, 1961, Monkey 5435 was prepared for operation and a suboccipital opening was made in the skull, the dura was incised and reflected, and the posterior vermis entered in the midline. This part of the vermis was subtotally removed. The animal was awake at the end of the operation and in good condition, but his head had a tendency to tilt backward and there was a nystagmus in all directions of gaze in which the eyes were turned, but none when he looked straight ahead. The animal had a wide base, with the hindlegs somewhat bent. During the remainder of August, the animal's condition was relatively unchanged except that his head assumed a more nearly normal position, although it showed peculiar movements as if his equilibrium were unstable. He still had a crouched gait and was definitely hypotonic.

During September, the behavior of the animal stabilized to some extent, but the peculiar head movements still persisted. He maintained a wide base on sitting or standing and his hindlegs were bent when he walked. The hypotonia was still demonstrable, but there was no evidence of nystagmus in any direction of gaze. On October 10,
1951, an operation on the cerebral cortex altered the clinical picture.

\section{Lesions in hemisphere(s) of posterior lobe}

Removal of small portions of the cerebellar hemisphere produced no overt signs in the monkeys studied wherever the lesion was placed. Not nearly all the areas of the cerebellar hemisphere were studied in this fashion. Particularly the more lateral and the more dorsal regions were spared. If the small lesion was deep enough to involve the dentate nucleus, an action tremor appeared on the side of the lesion.

As the hemisphere lesion was enlarged, hypotonia and ataxia, with a falling toward the side of the lesion, became evident. There was some incoordination in voluntary movements and, in some instances (depending upon the site and sometimes the depth of the lesion), a nystagmus. A cerebellar tremor was evident in large lesions if the dentate or the interpositus nuclei were involved. In many of the hemisphere lesions, the forelimbs were more affected than the hindlimbs.

On August 22, 1961, the paravermal areas of the cerebellum were removed in Monkey 5431, without injury to the posterior vermis (fig. 18), the cerebellar peduncles or the rest of the cortex of the cerebellar hemispheres, but with some involvement of interposed nuclei so that an action tremor was evident as late as October 10 . The animal was extremely ill immediately following the operation and it was, therefore, difficult to determine whether or not he had hypotonicity or ataxia. By September 17, aside from a slight

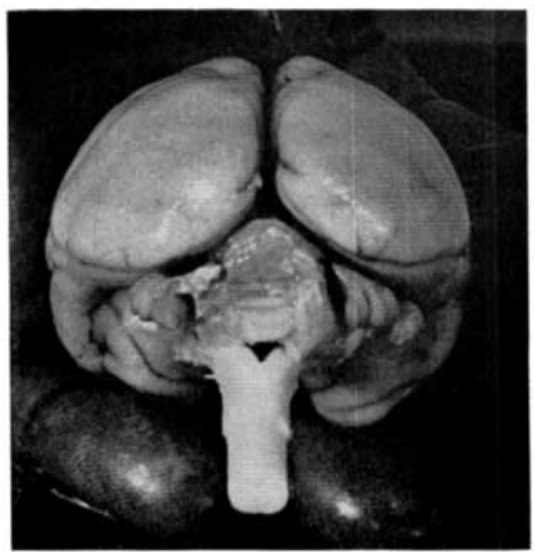

Fig. 18 Brain of Monkey 5431. 
action tremor, there were no cerebellar signs. The action tremor had disappeared by November 30 , and the animal could have passed for a normal monkey except for the scars.

On March 9, 1957, a suboccipital craniectomy was performed on Monkey 3994 and the right cerebellar tonsil and the immediately adjacent cortex and white matter of the inferior part of the right cerebellar hemisphere were destroyed. In the succeeding days, the animal recovered very quickly from the operation. A very slight hypotonia, present on the right on the first postoperative day, disappeared and no permanent effects of the cerebellar operation were demonstrated on any of the various examinations which were carried out. There was still no evidence of any difference in tone on the two sides of the body and no ataxia of the upper or the lower extremities on either side as late as April 11, 1957. At that time, the animal was used for other experiments.

Monkey 2001, when a similar operation had been carried out, had some incoordinate and ataxic movements of the right upper extremity. The site and the extent of his lesion were confirmed when he was sacrificed about one month postoperatively, on August 13, 1954.

On August 15, 1961, following a suboccipital cranicctomy, a large lesion was placed bilaterally in the hemispheric portion of the posterior lobe of the cerebellum in Monkey 5423. The lesions spared the lateral parts of each cerebellar hemisphere (as was confirmed on postmortem examination, August 8, 1963). Immediately after the animal recovered from the anesthetic, he showed a hypotonia of both upper and both lower extremities with the defect in tonus being greater for the upper than for the lower extremities. There was abnormal posturing of all extremities. Two days postoperatively, as the animal began to stand and move around the cage, the hindlimbs were bent and the forelimbs were far apart to provide a wide base. There were no involuntary movements, no tremor, and no nystagmus. During September and October of 1961, the animal gradually overcame most of the postoperative defects. His tonus became essentially normal and the crouched attitude disappeared. When tested on November 30, 1961, he showed no tremor, no nystagmus, no loss in placing, and had a normal tone. He walked in an essentially normal fashion and climbed very well. He was not sacrificed until almost two years after the cerebellar lesion was placed at which time (August 8,1963 ) he showed no cerebellar signs.

A suboccipital craniectomy was performed on August 9,1958, on Monkey 771. The cerebellum was exposed on the right, past the midline. The tonsil and the adjoining cerebellar hemisphere were removed by suction. Inadvertently the sinus was opened and considerable hemorrhage resulted. Following the operation, the monkey was cold and cyanotic but waked up readily. By the next day, he was alert and showed practically no effects of the operation except a slight swelling at the region of the incision. On August 11, the animal escaped while being caught for examination and proved very difficult to recapture, an indication of his excellent condition. On August 16, the hands were found to be exceptionally deft and there were no observable deficits. From this date until January 5, 1959, the animal was examined at regular intervals but no effects of the removal of portions of the cerebellum on August 9, 1958 were noted.

On August 2, 1960, the posterior fossa was opened and explored. The lesions placed in the cerebellum two years earlier were identified. The nodule, the uvula, and the pyramis were removed and the incision was carried over to the left side with an attempt to destroy the inferior cerebellar peduncle as it entered the cerebellum. The lesion was carried deep enough to destroy at least the caudal half (and possibly slightly more) of the fastigial nuelei and to invade the interpositus and the dentate nuclei.

In the days immediately following the operation, the animal gradually recovered but showed marked deficits. At first he was unable to get up at all from the bottom of the cage. By August 18 , he still could not stand or walk in an upright position but crouched on all fours and crept along the floor of the cage. He could climb the cage wall fairly well. He had a marked action tremor, most noticeable of the head.

On September 1, he still crept around the cage and could not stand erect. On this date he had a cerebral cortex lesion.

\section{Ablations of anterior lobe cortex}

None of the lesions involving the surface cortex of the anterior lobe produced permanent changes in tonus or any other persisting cerebellar signs. Dow and Moruzzi ('58) have also reported that the opisthotonos seen with surface lesions of the anterior lobe in carnivores was not obtained with similar procedures in monkeys. The effect of unilateral selective ablations of the medial cortex of the anterior cerebellar lobe in the cat is discussed by Chambers and Sprague ('51), and Sprague and Chambers ('53).

On October 17, 1961, after an occipital craniectomy with retraction of the occipital cortex and section of the tentorium cerebelli in Monkey 5583 , the cortex of the anterior lobe was exposed and this surface removed bilaterally and subtotally, but as completely as possible. There was essentially complete recovery. No visual loss was demonstrated due to the approach and, after the effects of the anesthetic had disappeared, there were no recognizable neurological signs. There were no effects of the operation during the succeeding two months up to the time the monkey was sacrificed (December 19, 1962). The location of the lesion was demonstrated.

In Monkey G574, on August 21, 1958, the left occipital lobe and part of the left temporal lobe 
were removed and the tentorium cerebelli cut to reveal the superior surface of the cerebellum. The superficial cortex of the anterior lobe was removed except for some remnants of this cortex bilaterally. The lobulus simplex was invaded but not quite completely destroyed on the left. There was an equivocal rigidity of the lower left extremity when the animal was recovering from the anesthetic but no trace of this was present the next morning. The animal moved slowly around the cage showing a slight ataxia on the side of the lesion and some posturing and jerky movements of the extremities on that side. There was a right homonymous hemianopia due to the destruction of the left occipital cortex. On the next day, the animal was fearful and combative and there were some evidences of ataxia and jerky uncoordinated responses to stimulation but no increased tonus. By August 31, the monkey showed no cerebellar signs and the only permanent deficit was the homonymous hemianopia. His condition remained unchanged until April 14 1959 , when he underwent a removal of area 6 on the right. When the monkey was finally sacrificed, on December 15,1959 , the position and the extent of the cerebellar lesion, as here recorded, were verified.

A lesion approximately the size of a quarter was placed on the superior surface of the left anterior lobe of the cerebellum in Monkey 5488 on September 14, 1961. In order to expose the cerebellar area, the left occipital cortex was retracted. In this retraction, an abrasion of the midlateral occipital cortex occurred and this injury was continued around the occipital pole onto the midcaudomedial part of the occipital cortex (fig. 19). The monkey showed some slowness in responding to objects brought into his right visual field, but certainly did not have a complete right homonymous hemianopia. The animal exhibited no overt cerebellar deficits after recovering from the anesthetic. The day following the experimental procedure, he moved around the cage easily, climbed well, and had no hypertonicity, ataxia, or tremor. None of these signs appeared during the succeeding months until he was sacrificed and the loca-

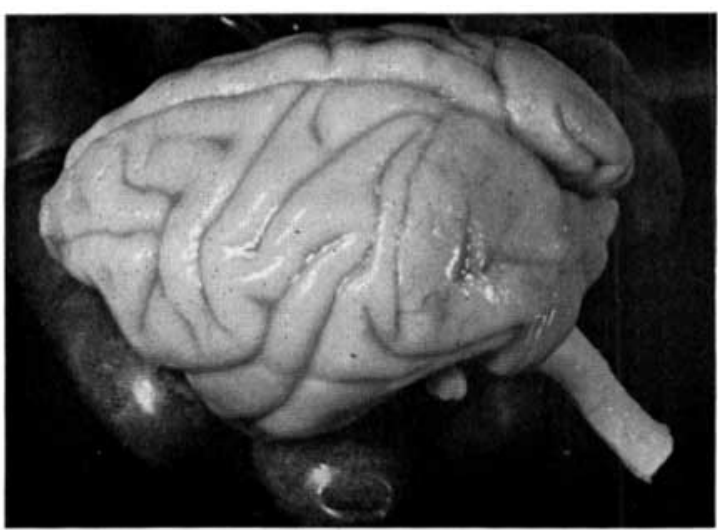

tion of the lesions verified by postmortem examination (July 7,1963 ).

Lesions of superior cerebellar peduncle(s)

Attempts to section the superior cerebellar peduncle, unilaterally or bilaterally, were not always successful. Out of the many attempts, only a few were partially or wholly satisfactory technically. Two methods of approach were tried. One method was to expose the occipital cortex by a parieto-occipital craniectomy, retract the occipital lobe, cut the tentorium cerebelli, and expose the superior surface of the cerebellum. The opening was carried as far laterally as feasible and the superior cerebellar peduncle exposed by retraction or by removal of the overlying cerebellar cortex. In either case there was some cerebellar cortex destruction and there was always some damage to the visual cortex by this approach.

A second method of approach was by way of the fourth ventricle. The posterior fossa was exposed by a suboccipital craniectomy and a laminectomy of the first three cervical vertebrae was performed, thus providing a larger field in which to work. The dura was then reflected and the lower part of the dorsal surface of the medulla and the inferior surface of the cerebellum were exposed. An incision was made longitudinally through the midline of the vermis and the cut edges gently retracted to reveal the floor of the fourth ventricle forward to the aqueduct. The ventricular surfaces underlying the superior cerebellar peduncles were located and

Fig. 19 Brain of Monkey 5488 
one peduncle or both peduncles sectioned by use of cauterization.

The results varied, with whichever method was employed, depending upon the exact site of, and the completeness of, the lesion in either, or both, peduncles. In general, incomplete sections of the peduncle involving more lateral and dorsolateral parts of it, such as were more frequently made with the occipital approach, appeared to affect the lower more than upper extremity on the side of the lesion. In an incomplete section on the ventricular side, which tended to be more medial and perhaps slightly ventromedial, the homolateral upper extremity, rather than the homolateral lower extremity, was usually involved. The extremity affected by the lesion showed hypotonicity, ataxia, and tremor. In a complete section of one superior cerebellar peduncle as it leaves the cerebellum (and so before its decussation), a marked hypotonia and ataxia on the side of the section involving the head, trunk, and extremities appeared. A monkey with such a lesion may lie for months on the operated side, too hypotonic to stand erect. The marked action tremor on the side of the lesion may abate considerably after some months, He often responds with jerky movements when touched and for a short time can set up a fair resistance to efforts to examine him so that he is less obviously hypotonic under such conditions than when his attention is diverted or he is at rest. After the immediate postoperative period, he is an alert, intelligent animal that will respond to any ameliorating circumstances, a very important point when attempts are being made to rehabilitate him. When the peduncles are sectioned bilaterally, the animal is prone on his stomach, can not stand erect even after a long period of time, and is ataxic and markedly hypotonic, with a persisting action tremor. $\mathrm{He}$ is still an intelligent animal. Partial bilateral sections handicap the monkey by producing a hypotonicity, an ataxia, and a tremor corresponding to, and in proportion to, the site and the extent of the lesions. If the injury is not too great, the animal may walk with a very crouched, wide-based gait.

In Monkey 5196 the right superior cerebellar peduncle was exposed by a suboccipital approach and, where it was emerging from the cerebellum, a small cut was made on its dorsomedial surface. This monkey showed a slight incoordination in the movements of his upper extremities, particularly on the right. This persisted as a very slight lack of deftness of the right hand from the time of operation (July 14, 1960) through January 16, 1961. The animal died February 16, 1962, from injuries received while attempting to escape from his cage. Postmortem examination indicated almost no injury to the superior cerebellar peduncle on the right.

On July 7,1959 , following a left parieto-occipital craniectomy and a reflection of the dura in Monkey 4887, the occipital lobe was exposed and retracted, the tentorium was excised to expose the cerebellum, and, after the trochlear nerve had been identified, the lateral part of the cerebellar hemisphere was ablated to reveal the superior cerebellar peduncle. Beginning laterally, the peduncle was cut through for about half to two-thirds of its width. The animal survived the operation very well but dragged around the cage by his hands because of the hypotonicity and the buckling of the left leg. By July 23, he was walking on all four extremities, but favored the left leg. He improved slowly, during August and September, and, by October 6, was quite active and, except for a slight hypotonicity of the left leg, showed no effects of the lesion. He was then used for another series of experiments, on January 14,1960 , and he was sacrificed on June 6 , 1961. The postmortem examination showed that although there was injury to the left superior cerebellar peduncle, the cut was not nearly so deep as had been supposed at the time of the operation. Perhaps this explains why only the leg was involved.

On March 4, 1962, after a left occipital craniectomy on Monkey 5568, the occipital lobe was retracted; the tentorium cerebelli was cut. The left brachium conjunctivum was identified and partially severed. Immediately after he recovered from the anesthetic, the monkey lay on his side in the cage apparently unwilling to stand or even to move. When disturbed, he showed a marked action tremor. After about a week, the animal began to drag himself around the cage and to try to sit up by leaning against the cage wall, but he could not walk. By March 8 , he sat most of the time against the side of the cage and, if disturbed, attempted to climb. He moved across the cage by leaning his uninjured side against the wall but his left hind limb was greatly bent and highly hypotonic. On May 8, there was marked hypotonicity of both lower extremities, with the greater hypotonicity on the left. During an attempt to get pictures, the monkey developed an epileptic attack. An electroencephalogram was made immediately and an increased discharge appeared in the rostrotemporal region, particularly over area 22. Another attack developed two days later when the animal was removed from the cage. The animal was sacrificed on May 17, at which time the cerebellar signs were essentially those reported on May 8. 
By the suboccipital approach, on July 2, 1959 , the left superior cerebellar peduncle was sectioned in Monkey 4711 . It was realized at the time of operation that the surrounding white matter was injured. The animal recovered quickly from the shock of the operation and was ready to be fed the next day. However, she could not stand but reclined on her side. When examined on July 5, she showed marked hypotonicity of the left extremities with somewhat more marked involvement of the lower than of the upper extremity. There was some weakness of the right lower extremity but much less than that on the left. There was an action tremor on the left but it was not much af a problem, perhaps because the animal pulled herself around the cage by her right arm and by shoving with her right leg and so did not use the left extremities. The head was tilted with the chin toward the left. The eyes showed some tendency to drift upward, as if the tonus was greater in those muscles that raised the eyes than in those that lowered them, but this inequality disappeared in a few days. It suggests, of course, the irritation of the flocculo-oculomotor path.

There was an ataxia and a past pointing as well as the marked hypotonicity on the left. Through the succeeding months, the animal was repeatedly checked but showed very little change and was unable to stand erect. Before the operation, she had been the ruler of her cage, eating first and dominating the other monkeys. In her incapacitated state, she was still such a tyrant that her cagemates obeyed in all matters of cage routine. Some six months after the first operation (on January 7, 1960), the skull was trephined on the right, the dura turned back, and the cortex removed subtotally from the right cerebral hemisphere.

On July 21, 1960, the posterior fossa was opened, the dura cut, and the vermis split down the midline to the level of the uvula. The two portions of the vermis were retracted and first the right, and then the left, superior cerebellar peduncle was severed in Monkey 5175. In an earlier attempt to implant an electrode in the left parietal area, a small infection had apparently involved the hand area so that the monkey had a loss of positional sense on the right upper extremity which must be taken into account in assessing the results of the cerebellar operation.

Following the operative procedure on July 21 the monkey had bilateral hypotonia (more marked on the left than on the right), a nystagmus to the left, and the head tilted toward the left with the chin toward the right when not supported. An action tremor was present bilaterally. He tended to rotate toward the right. The animal could not walk, although he attempted to get up on four feet. On September 7, 1960, an operation on the left cerebral hemisphere was carried out.

In addition to the monkeys thus far described, various other monkeys had partial lesions of the superior cerebellar peduncle. Since the results were largely confirmatory of those already given, they will not be reported. However, some interesting features are evident in comparing the effects of the lesions on Monkeys 5540 and 5096 . Monkey 5540 had had a partial destruction of the dorsolateral part of the superior cerebellar peduncle, which had produced an ataxia and hypotonicity most marked on the homolateral lower extremity. For four days, he maintained an upright position only by leaning against the cage, but he recovered rapidly and, within ten days, showed no obvious defects except possibly a slight hypotonicity in the lower extremity on the side of the lesion.

On July 14,1960 , the posterior fossa was opened widely in Monkey 5096 and both vermis and cerebellar hemispheres were well exposed. The vermis was removed until the lingula could be seen. Part of the posterior lobe of the left hemisphere and all of that lobe in the right hemisphere were destroyed. The right superior cerebellar peduncle was then cut along the floor and forward and upward to the anterolateral margin of the fourth ventricle. The lesion was carried well lateralward.

The monkey recovered quite satisfactorily from the immediate effects of the operation. On July 15, he showed a marked action tremor and marked hypotonicity, bilaterally. $\mathrm{He}$ could not walk but leaned up against the cage. By the following day he was beginning to move around the cage by crawling or with a very crouched posture. He showed marked action tremor and marked hypotonicity, especially on the right. This condition persisted, with only slight improvement, during the remainder of July. On August 1, 1960, he still had the same crouched posture and still crawled about the cage. There were marked tremor and hypotonicity, rather more on the right. An ataxia was apparent especially on the right. This condition persisted for a long time, although the tremor lessened somewhat.

The monkey was sacrificed on August 6, 1963, over three years after the operation. At that time, he had a hypotonicity, particularly of the right lower extremity, an ataxia, and an unsteady gait. When excited, there was a questionable tremor.

\section{Lesions of vermis and cerebellar hemisphere $(s)$}

When the cortex of half of the vermis and of one cerebellar hemisphere was removed, the animal tended to fall toward the side of the lesion and had a hypotonicity and an ataxia on that side. These signs became bilateral if both hemispheres and all of the vermis were involved even though the deep nuclei were spared. Animals with very large involvements of the cortex of the vermis and the hemispheres often walked again, but had an unusually crouched, wide based gait and marked hypotonicity. These defects in gait persisted to some degree for two or more years after the operation. Since none of our operations of this type had completely de- 
stroyed all of the cortical areas with sparing of the cerebellar nuclei, it is not possible to say whether the monkeys could have walked again if complete cortical destruction only had occurred.

None of the monkeys bere reported had only the cerebellar nuclei partially or wholly destroyed. The nuclear involvement was in conjunction with lesions of other portions of the cerebellum or of the cerebellar peduncle(s). When the dentate nucleus was invaded, an action tremor appeared on the side of the involvement. In a slight lesion of the dentate nucleus, the tremor was not very marked and did not persist. Greater destruction of the dentate resulted in more marked and more lasting tremor, the amount and persistence of the tremor being related to the extent of the nuclear involvement. In bilateral destruction of the dentate, the tremor was more than twice as great as after unilateral ablation of the nucleus and still more persistent.

In Monkey 5484, most of the vermis and the left half of the posterior cerebellar lobe with part of the underlying nuclear gray were removed on August 1, 1961. Following the operation, the animal was very hypotonic. He lay on his left side and could not stand upright or walk. The hypotonicity was present in both lower extremities and, at this time, about equal on the two sides. The left arm was decidedly more hypotonic than either the right arm or the left leg. A very coarse action tremor was present on the left whenever the animal attempted to move.

By August 10 , the animal could move around the cage but with a very crouching stance. He had a wide base, with now a more definite hypotonicity in the left leg than in the left arm and a tendency to fall toward the left. There was an action tremor of the left hand, although not very marked. By November 30 of the same year, the tremor had disappeared. The left hindleg was still decidedly weak and the body dropped toward the rear. The animal walked with his legs far apart but climbed well. There were no sensory losses, no lack of placing, and no demonstrable hypotonia except in the left leg.

During the succeeding years, the monkey showed very little change. His body continued to slant toward the rear and he still had a decidedly crouched gait. During September, 1963, the animal disappeared and his fate is unknown.

There was a subtotal removal of both cerebellar hemispheres and of the vermis in Monkey 2015 on March 15, 1956. Following this procedure, the monkey had a crouched gait, incoordinate movements, and an ataxia but no tremor. There was no dentate nucleus involvement. He remained relatively unchanged until May 12, 1956 when he had a removal of the cerebral cortex on the left.

Following widespread destruction of the cortex of vermis and cerebellar hemispheres (on October 28 , 1954), Monkey 2002 had marked ataxia, in coordination of movements, tremor, and a hypotonicity sufficiently great so that he was unable to stand up to November 1. During the next ten days he managed to get up on his feet but he walked with a very wide base, had a marked incoordination of the movements of all extremities, and had a head tremor at intervals. He could do very little with his upper extremities.

The animal was returned to his owner in December but, when he was sacrificed, the brain was examined and the results reported. In addition to an artifactual lesion on the inferior surface of the left temporal lobe there were large destroyed areas in both cerebellar hemispheres, in the anterior and the posterior lobes. The Hlocculi were spared and some of the upper part of the vermis. The inferior vermis and some of the superior vermis were destroyed (fig. 20).

\section{Cerebellectomies}

Two monkeys survived, for some months, total or subtotal removal of the cerebellum. Both of these monkeys were never able to walk again after the operation. They both had marked hypotonicity and very great action tremor. They crawled along the animal room floor with much effort and could not maintain an erect posture. Nevertheless, they were intelligent animals, showing none of the mental incapacitation and indifference to their state which characterized the monkeys with bilateral removal of the cerebral cortex.

On September 10,1963 , the cerebellurn was removed in Monkey 5729 as completely as possible, except for the flocculi which were subtotally de-

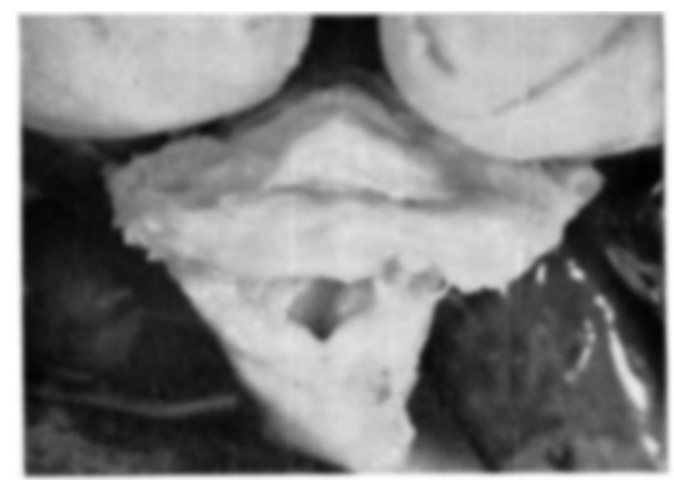

Fig. 20 Brain of monkey 2002 demonstrating the spared superior part of the vermis. 
stroyed in place and certainly cut off from all connection with other parts of the brain. For several days after the operation, the animal was very ill. He lay prone in the cage. Any attempt on his part to move set off an exceedingly marked action tremor of head and body. He could not feed himself and it was very difficult to feed him because of the tremor. Because of his poor condition, no examination was carried out for four days, postoperatively. By that time, although the tremor persisted and was so great that the animal injured his face by knocking his head against the cage wall when he tried to climb, he was trying to crawl around the cage floor and up the wall. He was unable to stand because of the great hypotonicity, but he crawled (fig. 21) around the floor of the cage of the animal room with his head slightly raised, since his forelimbs were slightly less hypotonic than his hindlimbs. He was apparently able to place but it was very difficult to test placing accurately because of the action tremor. He had no motor loss and responded to tactile and painful stimuli. Vision was unimpaired.

During the next month he changed very little except that he became able to feed himself somewhat less than well (his efforts were supplemented) and moved around a little more readily. However, the action tremor and the hypotonicity persisted and the head was particularly unstable. He had an excellent appetite but still appeared to be Iosing weight, apparently worn out by the tremor. This monkey was experimented upon again in the hope of lessening his tremor on October 17,1963 .

A suboccipital craniectomy and a cervical laminectomy at $\mathrm{C} 1$ and $\mathrm{C} 2$ were performed on Monkey 5646, on December 2, 1963. The cerebellum was removed as completely as possible except for the flocculi, which were destroyed in place. Later the same day, the animal was hypo-

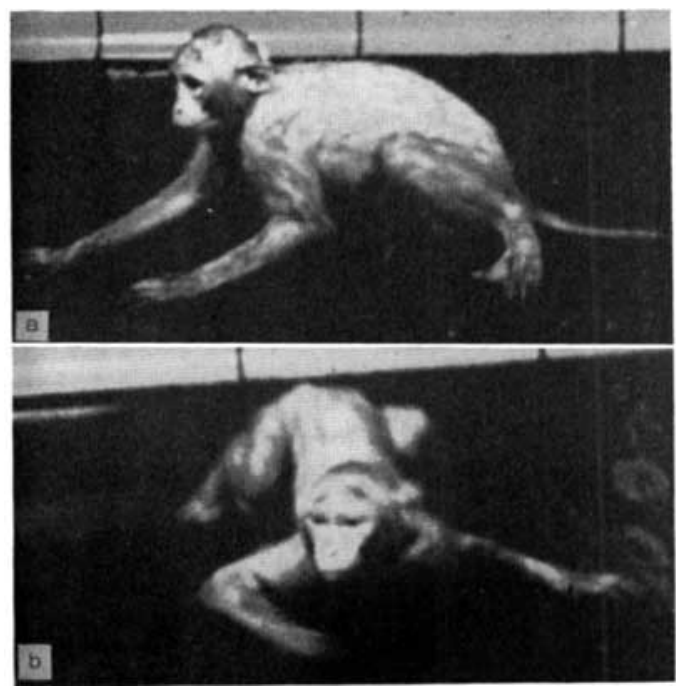

Fig. 21 Monkey 5729 on October 3, 1963. tonic, prone on the cage floor and exhibited a marked tremor of head and body if he were disturbed. He showed little change for the next ten days (fig. 22). When tested on December 14, he tried to drag himself for short distances on the cage floor and, if placed on the floor of the animal room, attempted to crawl along flat on his stomach with his head propped up on his arms. There were no motor deficits and he grasped well a bit of food placed between his fingers but he had such a tremulousness of both his body and his head that he could not place the food in his mouth although he tried desperately to do so. It was a problem to feed him because of the tremor, and he was partly fed by tube. He had a good appetite but still appeared to be losing weight and to be exhausted by the tremor. He changed very little during the ensuing month but he began to develop contractures from lying for such long periods in one position (fig. 23). Consequently, he was placed in a hammock with his legs suspended, a procedure to which he objected violently. Then one of the laboratory staff, John Demeter, made the barrel illustrated in figure 1 and the monkey learned to walk up and down the corridor in it. This contrivance helped to straighten the lower extremities and exercise them but did little for the upper extremities. Consequently, Mr. Demeter made the walker shown in figure 2 and the animal learned to use it.

\section{Combined involvements of cerebral} hemisphere(s) and cerebellum

Having produced a series of hypertonic monkeys from bilateral lesions of the cere-

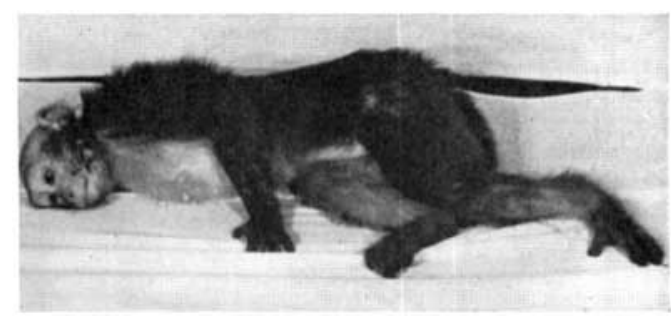

Fig. 22 Monkey 5646 one week postcerebellectomy.

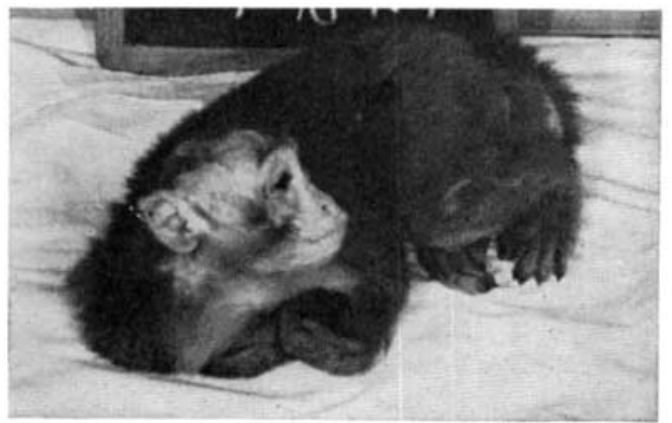

Fig. 23 Monkey 5646 six weeks postcerebellectomy. 
bral hemispheres and another series with large lesions in the posterior lobes of the cerebellum or with cerebellectomies which showed marked decrease in tonus, it was now proposed to attempt to create a hypotonicity or a hypertonicity experimentally and then to balance it by lesions in areas which had been shown to have an opposite effect on tonus. Thus lesions in the cerebral hemispheres producing handicapping hypertonicity were followed by lesions in cerebellar areas which previous experiments had shown gave handicapping hypotonicity. Then the reverse type of experiments was carried out with a gross lesion of the posterior lobe of the cerebellum or a hemicerebellectomy or almost total cerebellectomy followed by extensive cerebral cortex destruction.

The results of the experiments favored the predicted balancing between the cerebellar and the cerebral cortex discharges for the regulation of tonus. Monkeys, hypertonic from bilateral cerebral cortex involvements or some deep unilateral lesions involving cortex, portions of basal ganglia and deep white matter, approximated a normal tonus, or were even slightly hypotonic, after large, appropriately placed cerebellar lesions. On the contrary, a monkey which had been unable to stand erect for about five months (but lay on the involved side) as a result of section of one superior peduncle assumed an erect posture and even walked again (although very poorly) after a less than complete removal of the contralateral cerebral cortex.

The difficulties inherent in these attempts at balancing are more easily listed than solved. The first and foremost problem lies in the difficulty of equating the sizes of the lesions. Even when in an animal the primary lesion is placed with the greatest care, petechial hemorrhages, edema with softening, unsuspected vascular insufficiency, or the unrecognized sectioning of fiber paths in course may alter the expected picture. Like difficulties beset the placing of the secondary, supposedly balancing, lesion.

Another decided handicap is met in the limits to which cerebral cortex removal can be carried out and still leave a sufficiently intelligent animal to demonstrate the return of such functions as the ability to walk again. An alteration of tonus is more easily obtained than a return of useful function. It must be emphasized that, except as experimental evidence for the balancing of the discharges between cerebral and cerebellar areas in the regulation of tonus, destruction of large areas of cerebral cortex serves no useful purpose ( $p$. 15). The balancing of cerebral hemisphere lesions which have produced marked hypertonicity by appropriately placed cerebellar ablations may have some practical applications. It must be admitted that, in the series of experiments presented here, a perfect balancing of the discharges from the two regions has never been attained.

\section{In monkeys}

The case histories of some of the monkeys now to be considered as illustrating combined cerebral hemisphere and cerebellar lesions have been given in part in earlier sections of this report to show the effects of primary lesions in the cerebral or the cerebellar hemispheres. Where such is the case the material will be cross referenced and the present account will begin where the earlier one left off. In none of the monkeys in this series of experiments were lesions in both cerebral hemisphere and cerebellum made in the same operation except where portions of the occipital cortex were removed to destroy parts of the anterior lobe of the cerebellum or inadvertently in removals of the superior cerebellar peduncle.

At the beginning of October 1961, Monkey 5435 (p. 23) still walked with a wide base and with his hindlegs in a somewhat bent or crouched position, and still showed some peculiar head movements as if his equilibrium were not entirely stable, a residual from the destruction of the vcrmis on August 17. A parieto-occipital craniectomy was performed and areas $1,2,3,4,5,6$, and 7 were ablated on the right (October 10, 1961). During the next two weeks the animal was still unstable. He moved around the cage with a crouched stance and climbed poorly. This condition persisted into November. The hindlegs appeared somewhat more affected than the upper extremities, with not much difference between the right hindleg and the left hindleg with respect to tonus. Nevertheless he climbed and jumped somewhat better than earlier. His sensory loss, due to the removal of the somesthetic cortex, was a great problem. He had a minimal nystagmus in the horizontal plane on November 3. By November 30 , there was no nystagmus in any plane. There was an approximately normal tonus 
in the forelimbs. The left hindleg appeared weaker than the right and the animal maintained a wide base, particularly with his hindlegs, when he walked. Positional sense and placing were lost and there was some indication of a discriminatory tactile deficit on the side opposite the cortical lesion. There was also a loss of fine movements of the extremities on this same side. In the succeeding weeks he changed very little. On February 8,1962 , the animal showed slightly greater ease in walking but there was no fundamental difference in the general picture. The condition had stabilized and remained the same up to August 6, 1963, when he was used in another series of experiments. Postmortem examination on August 22 verified the sites and the extents of the lesions.

On July 21, 1960, Monkey 5175 (p. 27) showed an ataxia, rebound phenomena, and a hypo tonicity on the right. He had a peculiar gait and an unusual "tilting," crouching stance from earlier lesions of the superior cerebellar peduncles, together with some bilateral destruction of the vermis and of the right cerebellar hemisphere.

On September 7, 1960, a left frontoparietal craniectomy was carried out and areas 1, 2, 3, 4, 5,6 , and 7 were destroyed on the lateral hemisphere wall except that the face regions were spared. Postoperatively the animal was definitely more hypotonic on the left than on the right with the hypotonia more marked in the upper than in lower extremity. The somesthetic loss was particularly marked in the right upper extremity. He obviously did not know the position of his right hand and caught it in the bars of the cage and he lacked placing in the right upper extremity. These signs were somewhat less marked for the right lower extremity, which suggested some possible sparing in the paracentral gyrus. There was a nystagmus to the left. His head was tilted toward the left with the chin toward the right.

On October 27, 1960, an operation similar to that performed on the left cerebral cortex was carried out on the right side with destruction of areas $1,2,3,4,5,6$, and 7 . Postoperatively, the monkey showed hypotonicity and motor weakness bilaterally and, although he could move all extremities, he could not walk. He was sacrificed and the lesions verified on December 5, 1960 (fig. 24).

On June 18, 1959, Monkey 4755, who had survived a series of operations culminating in a bilateral cortectomy, the effects of which have been described earlier (p. 16), submitted to an operation on the cerebellum 43 days after the second cortectomy. The posterior fossa was opened, and as much as possible of the posterior lobe of the cerebellum removed. Later postmortem examination indicated that there had been sparing of the lateral and inferior part of the lobe bilaterally and that the flocculi had not been destroyed, although they had been cut off from the rest of the brain. After recovery from the anesthetic the animal did not cry so much as formerly, as if, with the reduced muscle tension, there was less pain. She ate well and seemed in fairly good health. The preoperative opisthotonos with contractures of both arms was still marked.

On June 20, 1959, the stiffness was less marked in the right lower than in the left lower extremity. The right arm was still bent at the elbow and the left arm was drawn up so that the left hand touched the face. There were contractures of both arms.

By June 26 there was a further relaxation. The left arm was withdrawn from the mouth; the right arm was at $90^{\circ}$ with the forearm but the left $\mathrm{arm}$ and forearm made a $120^{\circ}$ with each other. The knee could be bent quite well on the right and fairly well on the left.

In the period between June 26 and July 24 there was a gradual relaxation. The legs straightened out quite well and the arms were less stiff (fig. 25). However, the animal (lacking a cerebral cortex) was completely without desire to do anything and would make no attempt to feed herself or to crawl or walk around the cage, although when stimulated by tactile or other stim-
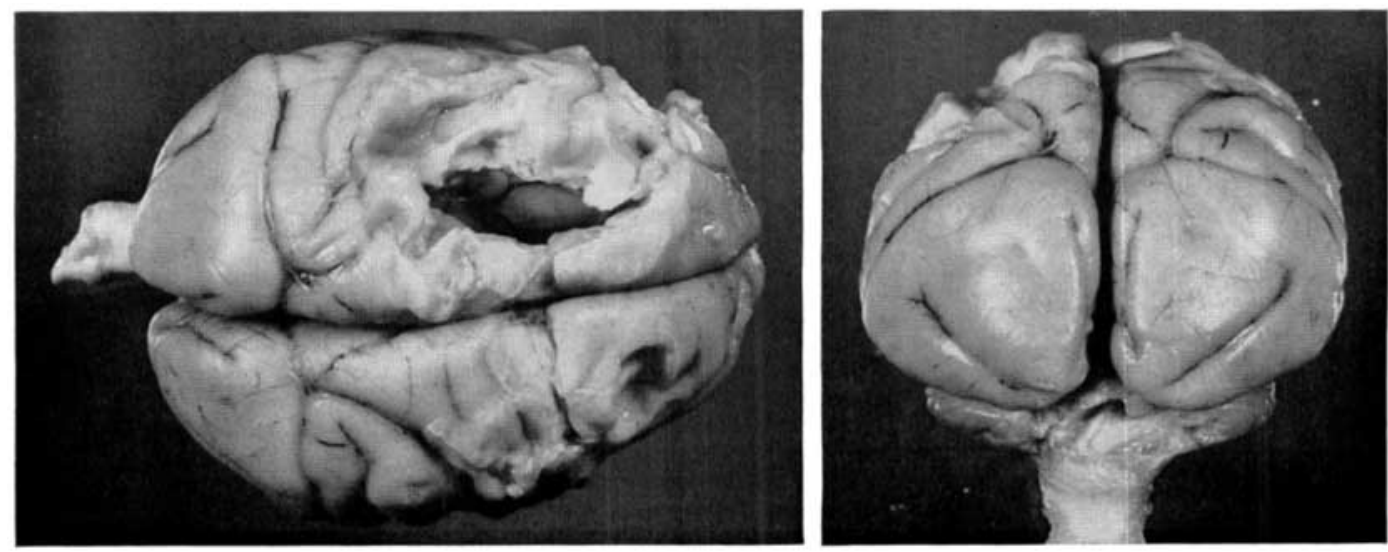

Fig. 24 Brain of monkey 5175. 


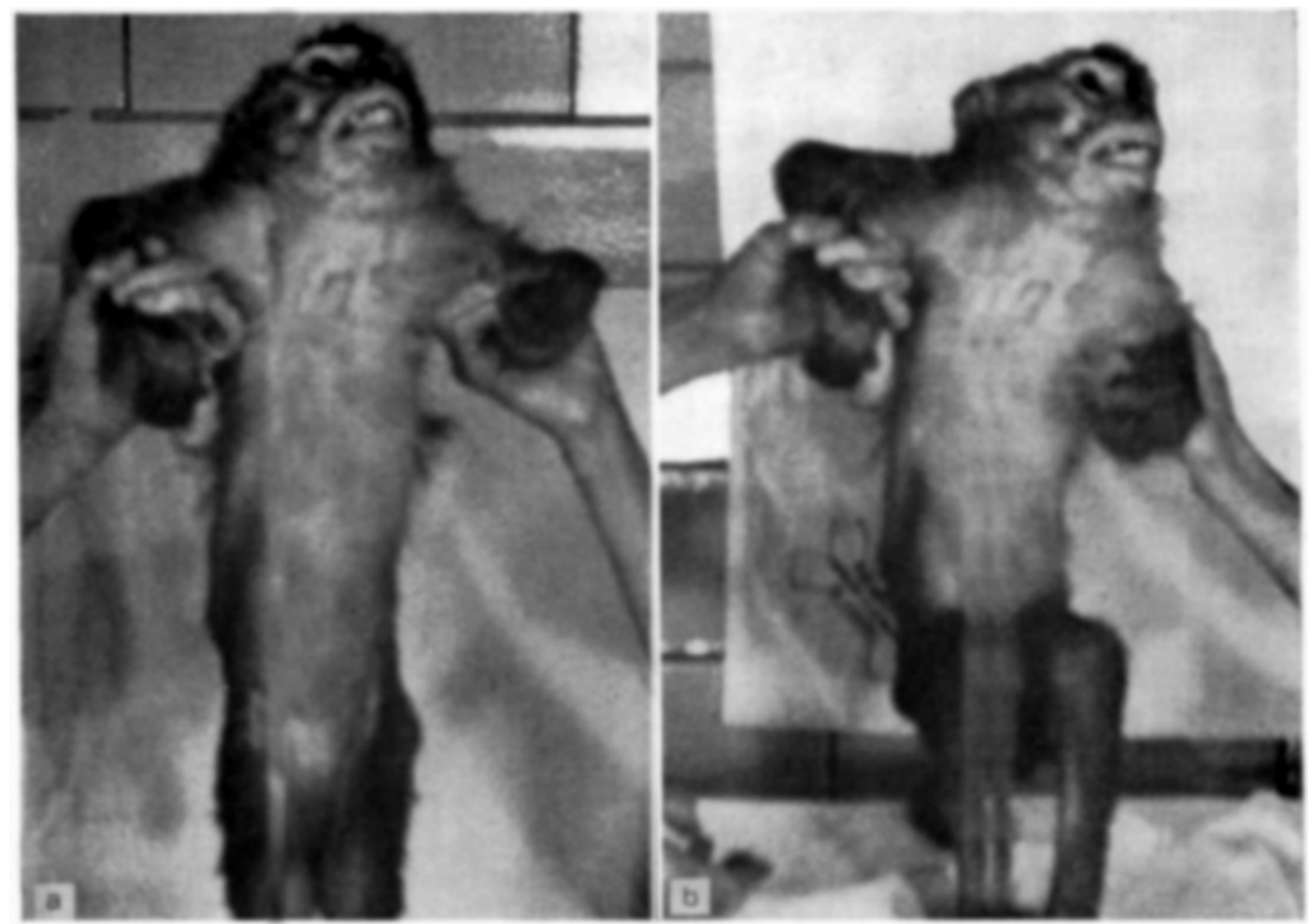

Fig. 25 Monkey 4755 on July 21, 1959.

uli, the legs showed movements suggesting she might have crawled if she had cared to try. The animal was sacrificed on July $24,1959,80$ days after the second cortectomy (fig. 26) and the brain processed ( $\mathrm{fig}, 27$ ).

On October 17,1963 , Monkey 5729, that had had ( $p .28$ ) a substantially complete cerebellectomy on September 10,1963, was again operated upon in the hope of lessening the incapacitating action tremor. Following a left frontoparietal craniectomy, the dura was retracted and the motor cortex located and stimulated to determine the motor pattern. The areas giving upper and lower extremity movements were ablated. The animal recovered very quickly from the operation.

When examined the next day after the operation, the tremor had disappeared bilaterally for body and extremities, but there was still a tremor of the head. The hypotonicity was slightly increased, especially on the right upper extremity, During the succeeding days, because of the loss of the tremor, the animal was better able to feed himself. He improved in health and apparently in spirits, but was still completely unable to assume any but a prone position. In about two weeks the tremor began to return on the side of the cortical lesion for the extremities and the body. It never became so excessive as it had been before the unilateral destruction of the cortex and it never returned on the side opposite the cerebral cortex ablation.

In the hope of still improving the monkey's condition, a right sided opening was made in the skull and a removal of the cerebral cortex on the right was attempted on December 1,1963 . There was considerable bleeding and the monkey was in a state of shock when he was returned to his cage. However, he was awake later that night and seemed to be doing fairly well. He moved when touched. There was no indication of tremor on either side of the body or on the extremities. The animal died the next day of a massive cerebral hemorrhage. The brain is shown in figure 28 .

At the time that Monkey 4865 was given to the group, she had had a subtotal removal of the left cerebral cortex with a sparing of the thalamus and the basal ganglia. These lesions were verified later on postmortem examination (fig. 29). On June 4, 1958, the vermis had been ablated. She showed a loss of fine individual finger movements and a loss of positional sense on the right, and had a right homonymous hemianopia as a result of the ccrebral cortex ablation. As a consequence of the cerebellar lesion, she walked with bent hindlegs and a wide base.

On May 19, 1959, a right craniectomy was performed and the cortex and the basal ganglia (caudate, putamen, and globus pallidus) de- 

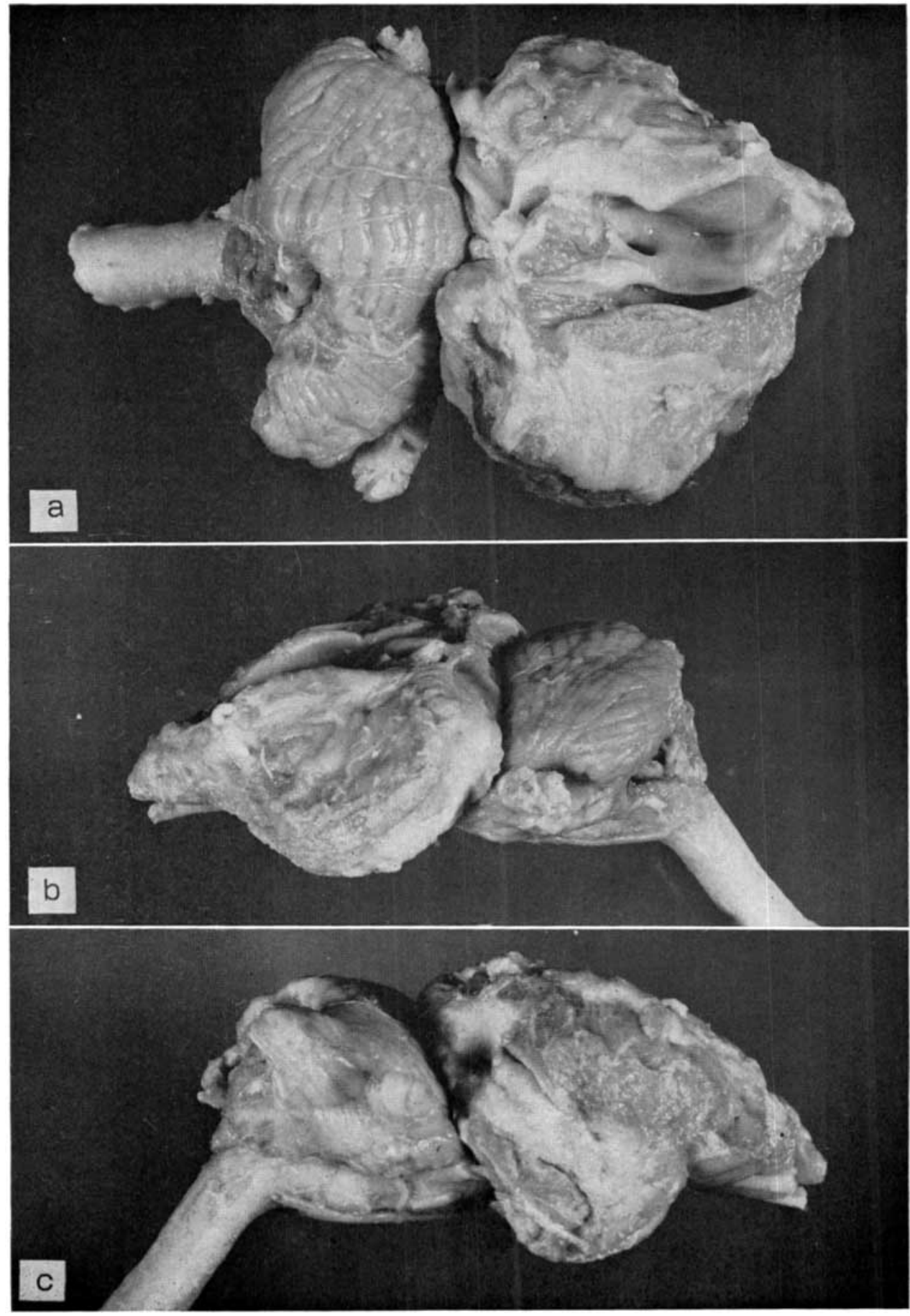

Fig. 26 Brain of Monkey 4755. 

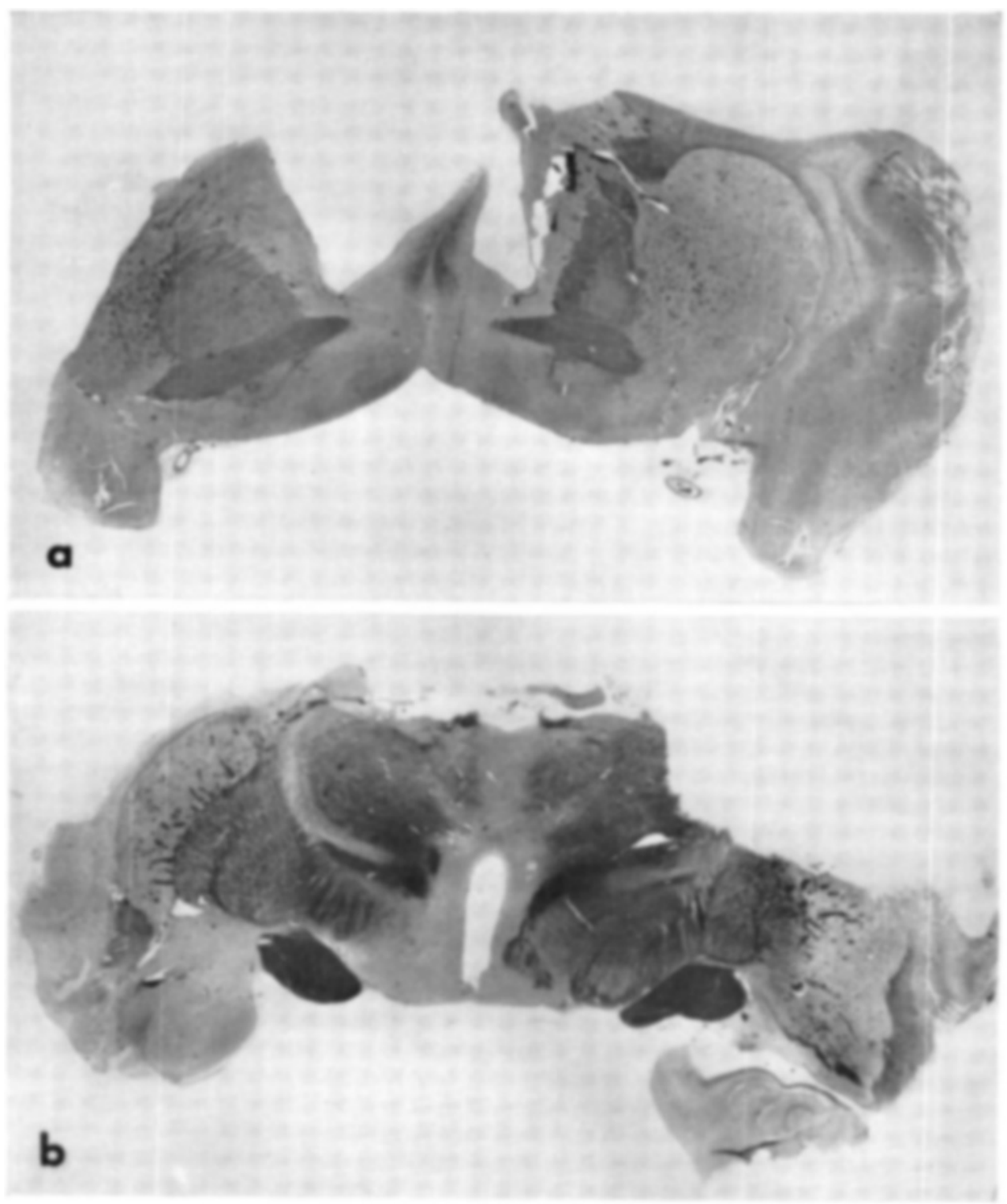

Fig. 27 a-b Photomicrographs of brain of Monkey 4755 to indicate the invasion of the basal ganglia by the lesion. (a) Section just rostral to the decussation of the anterior commissure. Caudate and putamen are invaded on the left and spared on the right, $3.2 \times$. (b) Section through rostral third of diencerhalon. Apparent extent of lesion into right internal capsule and basal ganglia is artifactual due to sectioning at the end of the Marchi block. On the left side the degeneration in the thalamic reticular nucleus is very evident, $3.3 \times$.

stroyed. Late that same evening, she was recovering consciousness. She was awake the next morning and moved when touched. There were some extension and weakness of the left leg.

On May 21, she crawled around the cage and showed jerky movements when touched. She was blind. She had no fine movements or placing of the extremities. She did not appear to be entirely deaf, since she jerked at sudden loud noises. She ate well.

By May 23, she had a fine trenıor when resting on the side opposite the last operation. She responded by jerky movements when touched on the side of the operation but had no tremor on 


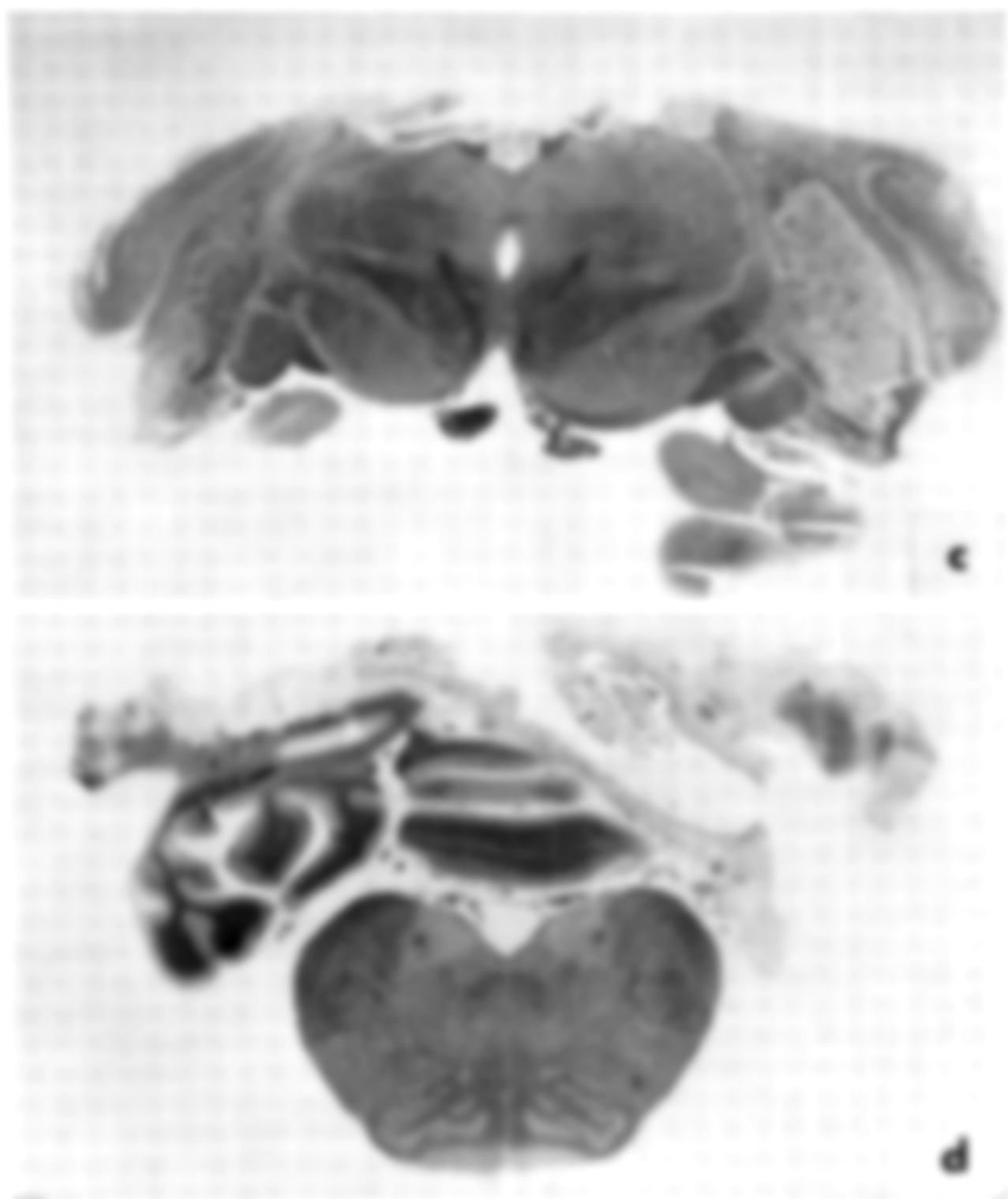

Fig. $27 \mathrm{c}-\mathrm{d}$ (c) Section through midbrain-thalamic transition showing the lateral geniculate nucleus and the oculomotor nerves bilaterally, $3 \times$. (d) Section through medulla at level of the Xth and XIIth nuclei rostral to the obex showing bilaterally degenerated pyramidal tracts and major degeneration of cerebellum, $4.4 \times$.

that side. The other findings were the same as those for May 21.

During the succeeding days, the animal gradually became stiffer. The legs were extended, the arms bent at the elbows and the wrists, and the hands clenched. The tail was extended although she could wag it slightly. Involuntary jerky movements of the head and of the shoulders appeared when she was touched. When she was rolled over, suggestions of an opisthotonos appeared, such as are seen in bilateral cortectomies. At rest, the lumbosacral spine was flexed. Although she showed, in general, hypertonia there were signs of an alternating hypotonia. It was possible to flap the right arm and the right hand. When partially supported in an erect position, she 


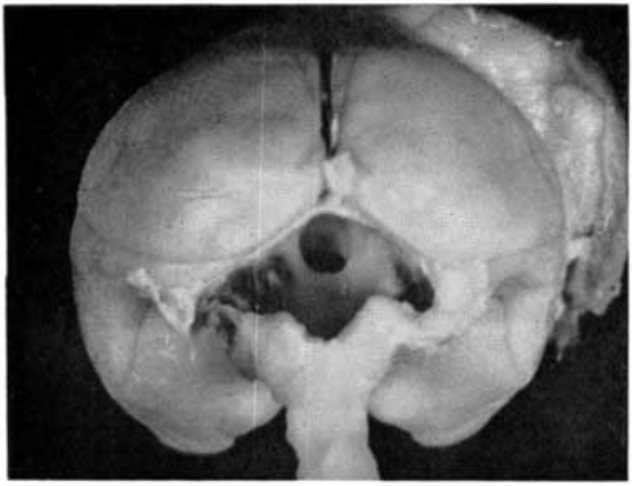

Fig. 28 Brain of Monkey 5729 showing the complete cerebellectomy.

showed some ability to support herself on her lower extremities, but less on her upper extremities.

Over the succeeding weeks, until the animal was sacrificed on July 24 , all sorts of attempts were made to get her to walk again. The hypertonicity, although always present, waxed and waned. She showed somewhat better extremity movements as time went on, especially on the right. The opisthotonos never developed.

In June she moved around a fair amount when placed in a sitting position. Actually the experi- menters felt that her absolute indifference to her condition (to be expected since she had almost no cortex left) was her insuperable handicap in learning to walk again. The blindness and the bilateral loss of positional sense and of fine movements persisted. She reacted to loud noises so that it was felt that she was not completely deaf but the noises appeared meaningless (Neff and Diamond, '58). She also retained gross tactile and pain sensibility,

Postmortem examination (fig. 29) showed complete destruction of the cerebral cortex, the claustrum, the caudate, and the lentiform nuclei together with the overlying white matter on the right. The thalamus was spared. On the left most of the cerebral cortex had been destroyed, but the uncus, the hippocampus, and all of the basal ganglia, as well as the thalamus, were spared. The vermis was destroyed, but the cerebellar hemispheres were not injured. The cerebellum was shifted slightly toward the left.

The motor and the sensory areas were exposed bilaterally in Monkey 732 on November 7, 1957, by performing a bilateral craniectomy. The regions (p. 6) related to various extremity and face movements were determined by stimulation and removed on the right and for all but the face area on the left. The monkey recovered very well from the operation and, in the ensuing days, walked and climbed around the cage. He showed a central facial weakness on the left, a loss of fine movements of the individual fingers and toes
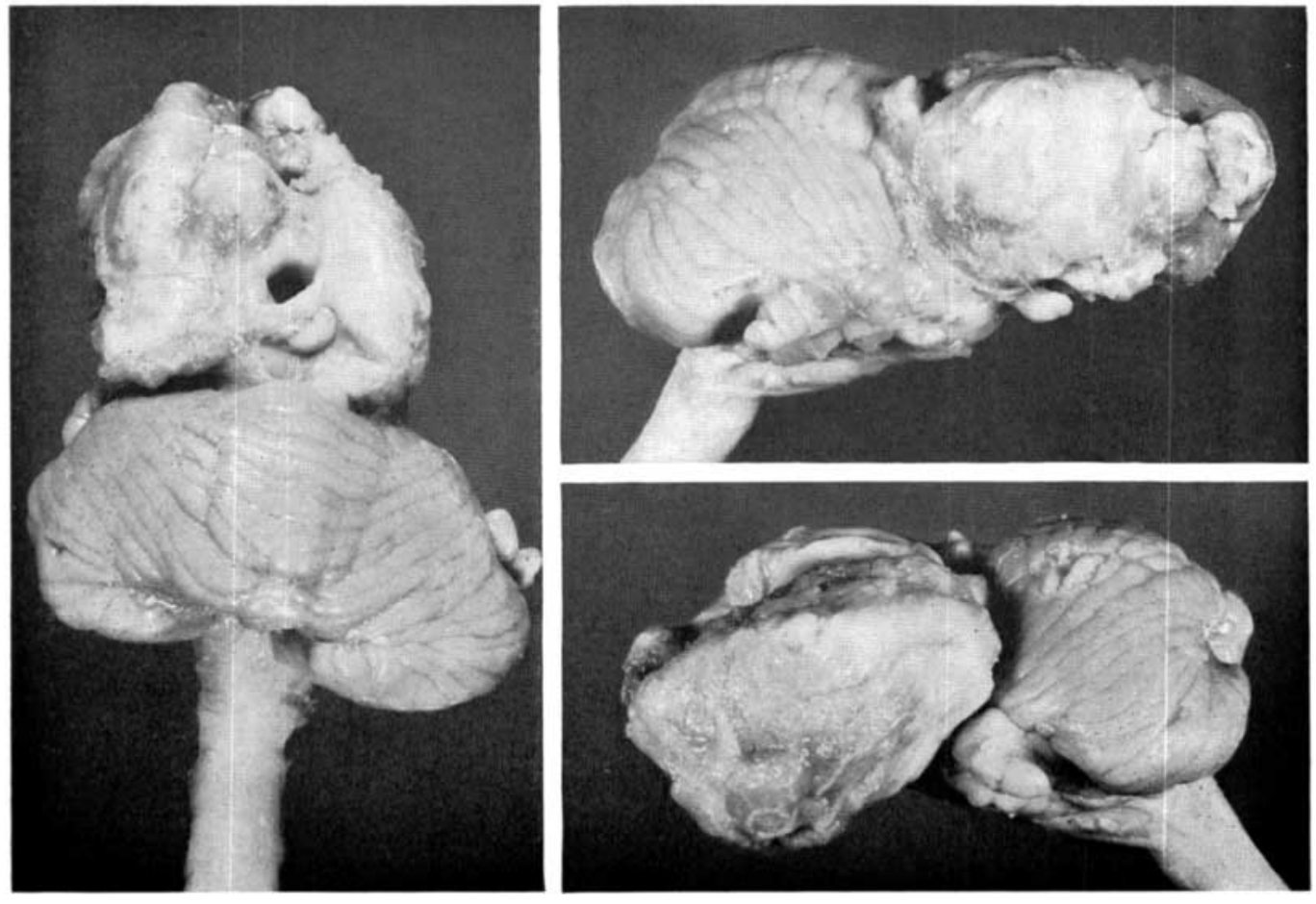

Fig. 29 Brain of Monkey 4865. 
on all four extremities, and a loss of positional sense on these extremities. He could not place. Tactile discrimination was not tested satisfactorily. On November 14, and again on November 21 , in addition to the preceding deficits, the animal showed a slight increase in tonus in the lower extremitics and his tail was extended.

On November 21 a left cortectomy was carried out. There was some involvement also of the caudate nucleus on the left. For the first postoperative day the animal lay on his side, but moved when he was touched. He recovered quite rapidly during the succeeding week, but had a distinct motor handicap more evident on the right than on the left.

On December 5, there was still deviation of head and eyes toward the left. However, he could turn his head to the midline and look straight ahead. He had a right homonymous hemianopia. His right arm was flexed and showed some contracture. The left arm had a paretic position when he was sitting quietly but it could be moved. His legs were flexed, but showed increased tonus. His toes were spanned, his tail erect, and he had a scissors gait. He ted himself with his left hand. There appeared to be a dim inution of tactile sensibility, greater on the right. Positional sense was gone bilaterally

On December 11, 1957, a suboccipital craniectomy was performed, the dura resected, and the left ccrebellar hemisphere exposed and subtotally removed on the left. In the succeeding ten days the animal recovered from the operation with a minimal upset. However, tests showed a diminution of tone on the left.

On December 31, 1957, the remainder of the left posterior lobe of the cerebellum was removed. Again the animal recovered very well from the operation and, by January 3 , appeared to be in quite good health. He could eat by himself but had difficulty or clumsiness in picking up his food. He moved around the cage quite well. Examination, on January 10 , indicated that following the last operation there had been a further decrease in tonus but the scissors gait remained. On January 19,1958 , there was quite a little less tonus on the left than on the right; the head was rotated and the right arm was held in flexion. The legs were flexed. The animal fed himself but was clumsy in grasping an apple placed in front of him. There was forced grasping with the right hand.

During the next month there was some decrease in the scissoring of the lower extremities, but the right lower extremity remained flexed and bent. When placed in water, the animal was able to support himself along the margins of the tub. During this period, baths, exercise, and careful nursing were employed with the hope of enabling the animal to walk again. In retrospect, one wonders why the right hemisphere part of the posterior lobe was not removed. On February 22 , the animal's legs were still in Hexion, but much looser from the calf down, and his right hand, now held in extension, could be used to support him. Throughout March and April, the animal remained definitely crippled and required much nursing care.
On April 10, 1958, Monkey 732 was able to flex his right hindleg and push himself along on his feet and toes. There was fairly good movement of the right upper extremity and he could reach out and take an apple. His head was turned toward the left and his back had a lumbosacral curvature, with the right side concave. There was a flexion contraction of the lower extremities, which was very incapacitating, but the animal was able to drag himself around the cage or along the wall by using his upper extremities.

On April 29, 1958, a right obturator neurectomy was carried out and two days later a similar procedure was done on the left. When examined two days after the second of these two nerve sections, the left lower extremity was somewhat abducted and the position of the right lower extremity had improved to some extent, so that the animal walked rather better. An attempt on May 4, 1958, to correct the curvature of the spine was fatal to the animal.

On September 1, 1960, areas 6, 4, 3, 1, 2, 5, and 7 were stimulated on the left in Monkey 771 , to identify upper and lower extremity regions and the arm portion and some of the leg portion were ablated to a depth of 2 to $2.5 \mathrm{~mm}$. The head areas were spared. This monkey had previously had a cerebellar operation (p. 24).

By September 2, the animal appeared to be in good condition and was already moving around the cage. A hypotonia was evident in all extremities. There was a lass of fine movements, particularly of the distal portions of the right upper extremity. There was also a gross inability to judge the position of the hand. The head was almost straight but still showed a gross tremor. During the next month, the condition of the animal improved slightly. When examined on October 6 (fig. 30) he had a hypotonia on the right and an inability to place with his right hand or to judge its position as he climbed the cage. There appeared to be little or no effect on the right foot. There was almost no head rotation but there was a noticeable head tremor.

An attempt. was made, on January 1, 1961, to remove the arm region of areas $6,4,3,1$, and 2 on the right, after these had been identified by stimulation but the animal was in poor condition after the operation and it was not possible to get any satisfactory tests. He succumbed on January 13, 1961.

A postmortem examination (fig. 31) indicated that, on the left, those portions of areas $6,4,3$, $1,2,5$, and 7 from which right upper extremity movements were elicited on stimulation had been ablated. On the right, those parts of areas 6,4 3,2 , and 1 giving movements of the left arm had been destroyed. In the posterior fossa, the flocculi had been spared. The inferior, the posterior, and the lateral portions of the right cerebellar hemisphere had been removed. The right side of the vermis had been destroyed and the lesion had been carried across the midline into the posterior vermis. Consequently, nearly all of the nodule, the uvula, and the pyramis had been removed. There was an involvement of the cerebellar nuclei bilaterally, with the dentate nucleus being slightly more involved on the right than on the left. 


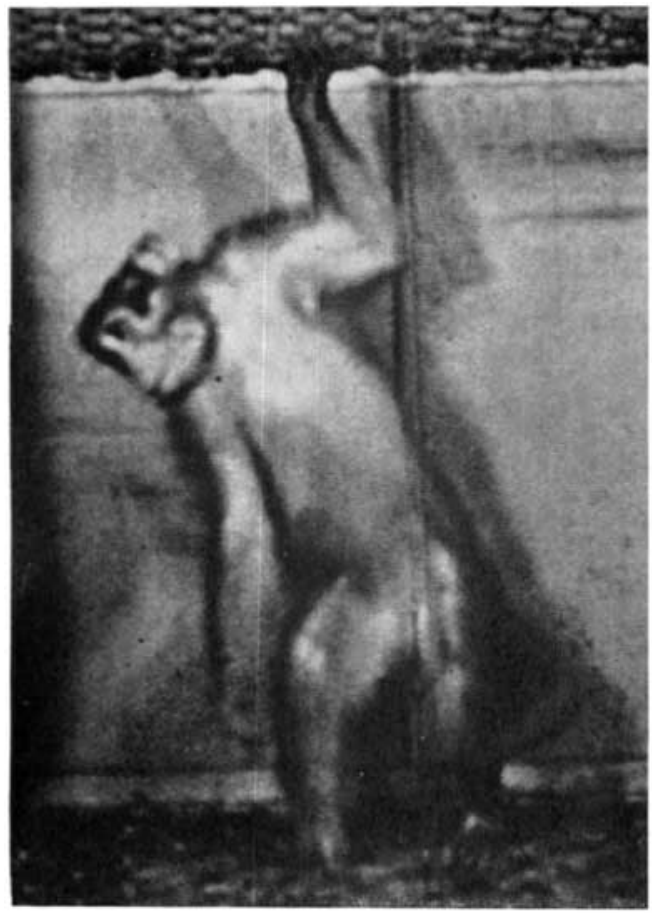

Fig. 30 Monkey 771 on October 6, 1960.

On January 7,1960 a right cortectomy was performed on Monkey 4711 which had previously had a left superior cerebellar pedunculotomy (p. 27). The monkey was in a somewhat critical condition immediately following the operation but gradually improved during the succeeding days. By January 10, 1960 she showed definitely less hypotonicity on the left than preoperatively. When taken out of the cage and placed on the floor, on January 11, she tried to right herself but was not very successful (fig. 32). By January 15 , she crouched on all four feet on the bottom of the cage. By January 17, the animal was trying to walk. She did not do badly, especially if she could lean against something. She had a crouched position and was still weak on the side opposite the cortical lesion.

During the next seven weeks the locomotion gradually improved to some small extent (fig. 33), although she was still greatly handicapped.

On April 28, the areas related to extremity movements were removed in the motor, premotor, and sensory areas of the left cercbral cortex. By May 2, the animal had recovered somewhat from her operation. She struggled but could not stand or walk. She was hypertonic and the hypertonicity increased during the next few days, so that by May 6 she was beginning to show an opisthotonos. The marked hypertonicity remained and she became a serious nursing problem. She was sacrificed on June 28, 1960.

The postmortem (fig. 34) showed that the cortectomy on the left was partial. It spared the hippocampus with fimbria and fornix, the amygdala, a considerable part of the temporal lobe, and some portions of the rostral end and medial inferior surface of the temporal lobe. Microscopic material (fig. 35 , a and b; fig. $36 a, b$ ) confirmed the degeneration in the superior ccrebellar peduncle, in the cerebellorubrothalamic system, and in the decussation of the superior cerebellar peduncles.

On January 23, 1964, in an effort to lessen the tremor which had diminished very little (p. 29), from the time of the cerebellectomy, the portions of the right motor cortex in Monkey 5646 related to upper and lower extremity movements were removed with a sucker. The animal recovered quickly from the operation. On Jamuary 24 , he was in good shape and the tremor had disappeared bilaterally. $\mathrm{He}$ was very hypotonic. By January 26, the head tremor was returning and, a day later, it was present on the side of the motor cortex destruction. It was less marked than preoperatively and never returned on the left extremities. He had a paresis of the right
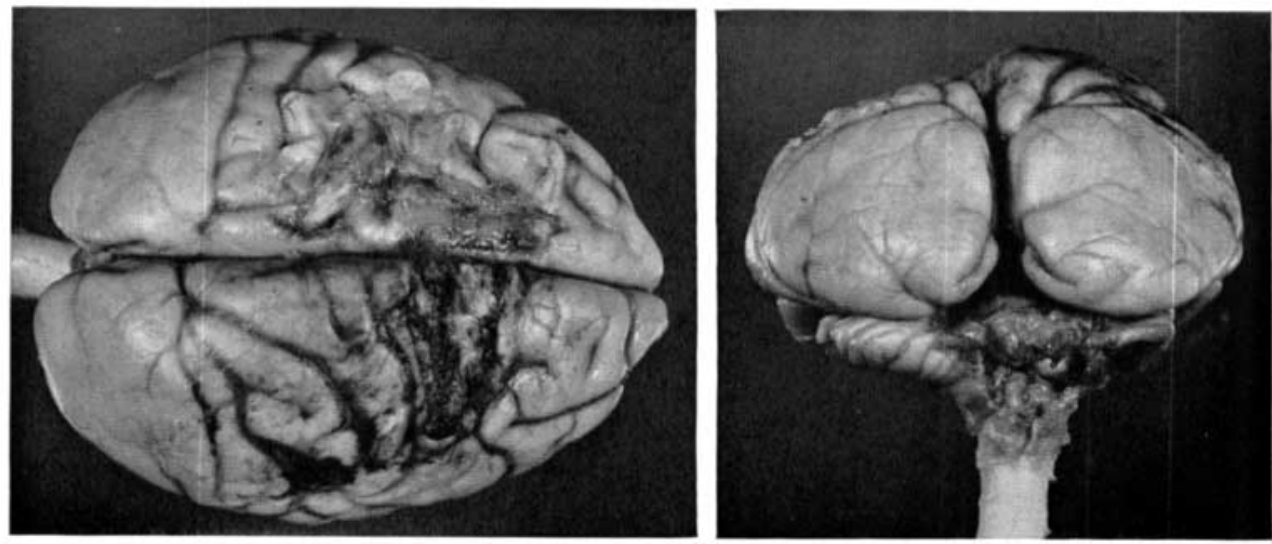

Fig. 31 Brain of Monkey 771. 

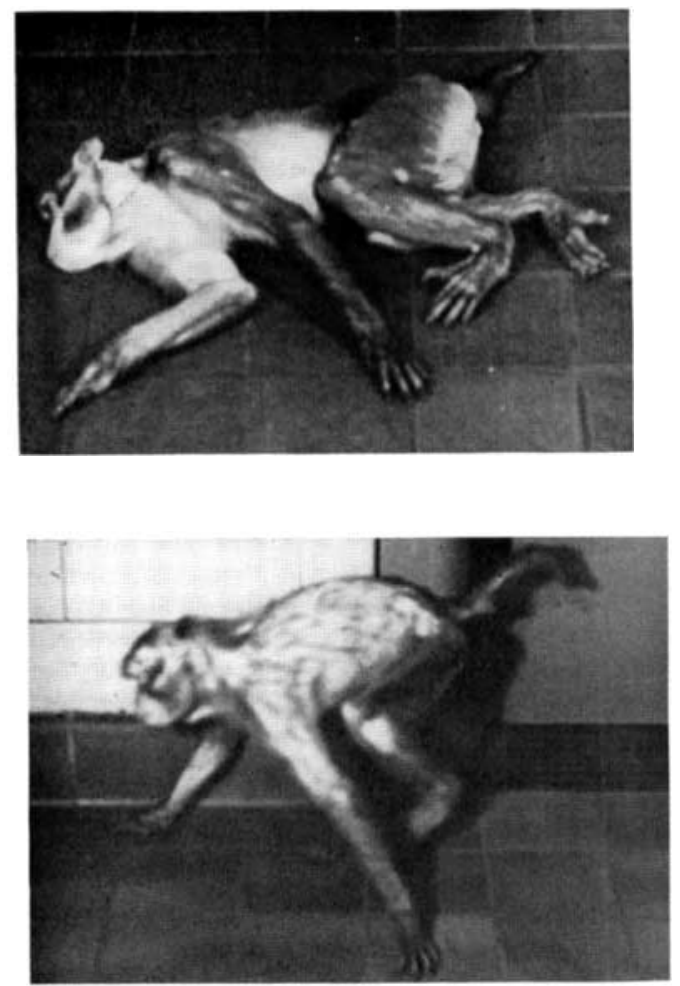

Fig. 33 Monkey 4711 on February 28, 1960.

upper and lower extremitics but could use his hand for eating. The animal learned to walk again with the walkers (fig, 37 ).

On March 16, 1964, the left motor cortex was sucked off in the region related to movements of the contralateral extremities. Again the monkey recovered very rapidly from the operation. Im- mediatcly fostoperatively, the tremor had entirely disappeared. It began to return for the head about 36 hours after the oferation and increased somewhat over the succeeding days but never regained its original violence. Otherwise, he gave no evidence of a tremor unless he became violently excited in which case there was an occasional trace of a tremor on the body or the extremities. He was unable to support himself on his extremities and also he needed support to sit in an erect rosition. His hypotonia was apparently greater than before the removal of the motor cortices. There were occasional short bursts of hyfertonia on testing. With the support of either walker, he moved up or down the corridor in search of candy or seeking to return to his cage.

On June 4, 1964, the previous opening in the skull was enlarged, the central fissure and the somesthetic cortex identified, bilaterally, and a lesion placed in both right and left hemispheres, in the portions of areas 5 and 7 from which lower and upper extremity movements can be elicited. The animal was awake when removed from the operating table. He was still very hypotonic and had an action tremor of the head. Nevertheless, the lower extremities showed a hyperextension ( $f$ ig. 38). He was allowed two days to recover from the effects of the operation and then placed again in the walker. At first he was not able to move it. Within a few days he was moving up and down the hall in the walkers, but not quite so readily as before the last operation.

From the middle of June until the middle of August, he showed little change. He had no useful increase in tonus. The action tremor of the head was slightly diminished but was still present and increased as the monkey became ex cited. He was sacrificed on October 6, 1964 (fig. 39).

\section{In children}

After many experiments on monkeys in an effort to produce hypertonicity and then
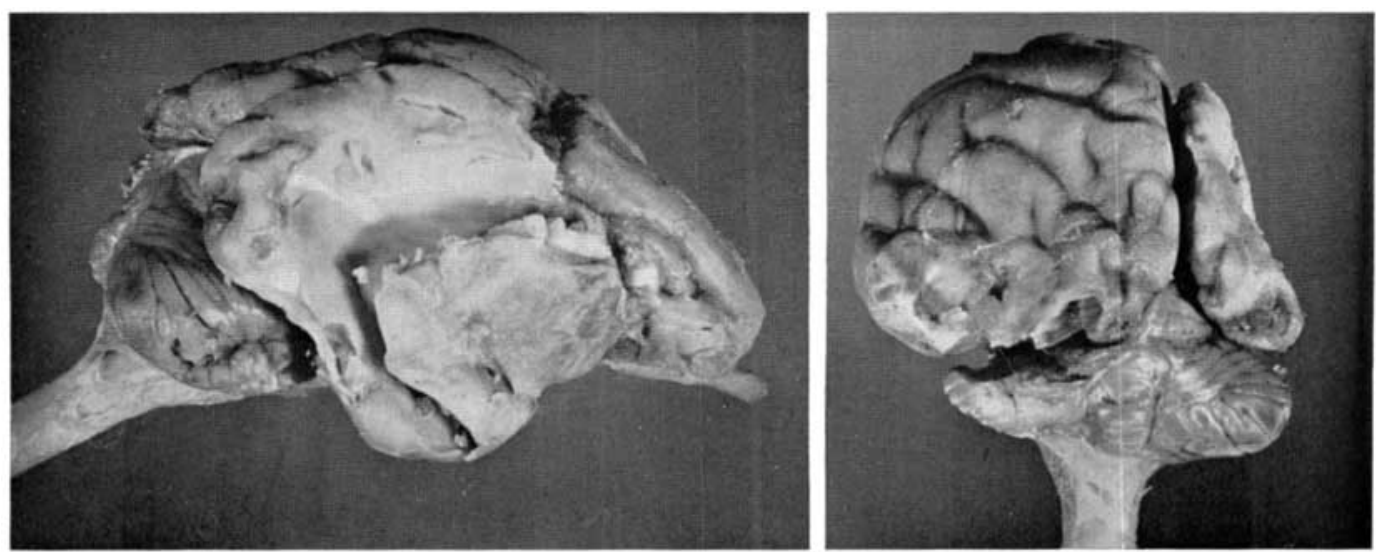

Fig. 34 Brain of Monkey 4711. (Reprinted by permission from the Editors of the Journal of Neurosurgery, from Schneider and Crosby, '63.) 

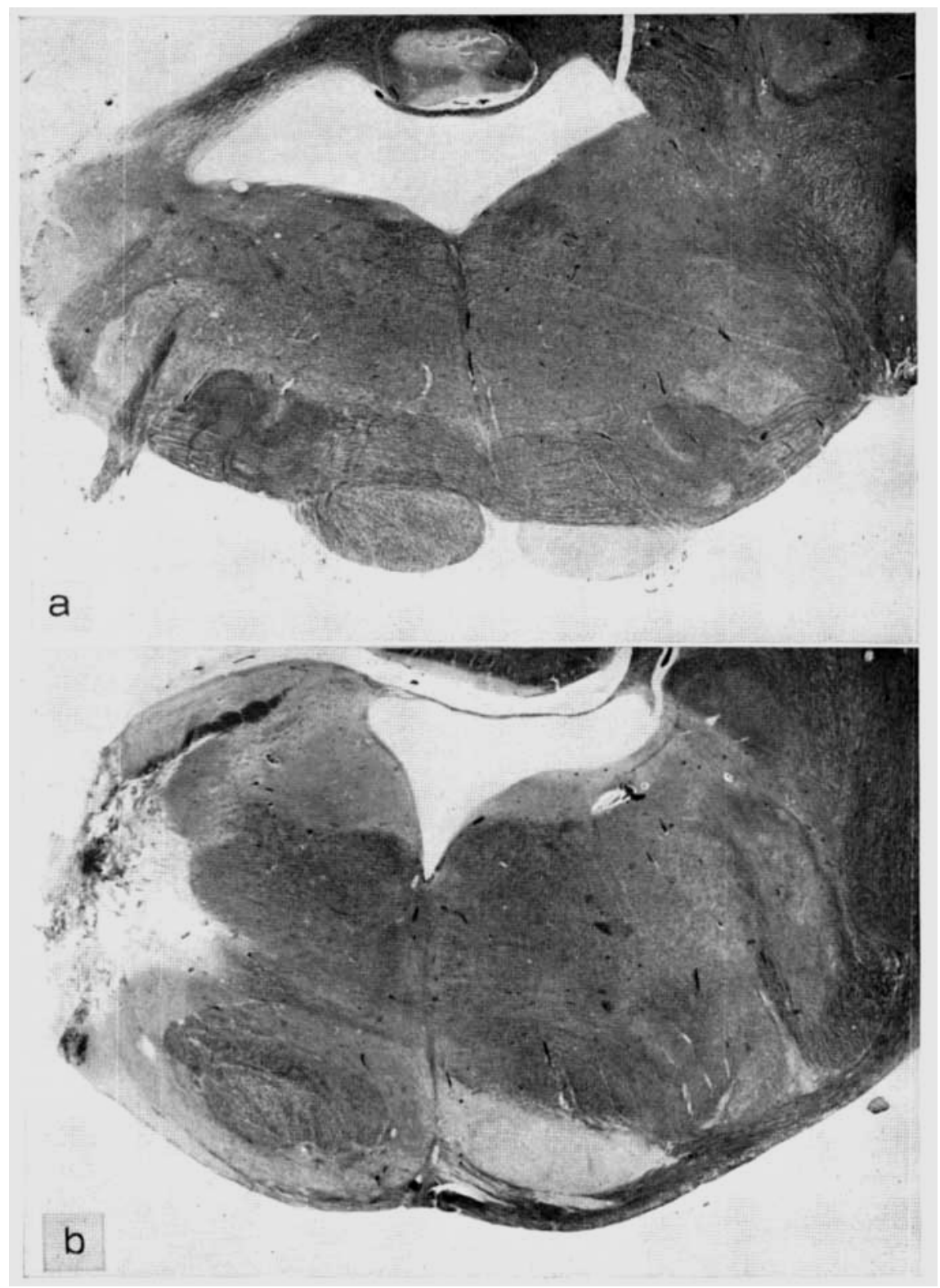

Fig. 35 Photomicrographs of brain of Monkey 4711. (a) Level of transition between pons and medulla with emerging left VIIth root. The lesion at this level invades the inferior and middle cerebellar peduncles and the dorsolateral border of the spinal tract of V. Complete degeneration of the right pyramidal tract is apparent, $6.5 \times$. (b) Section through the pons at the level of trigeminal root. The left motor and sensory trigeminal nuclei and associated spinal tract of $V$ are destroyed and the lesion at its maximum extends far enough medialward to get into the medial lemniscus. Again note the complete degeneration of pyramidal corticopontine systems on the right, $9 \times$. 


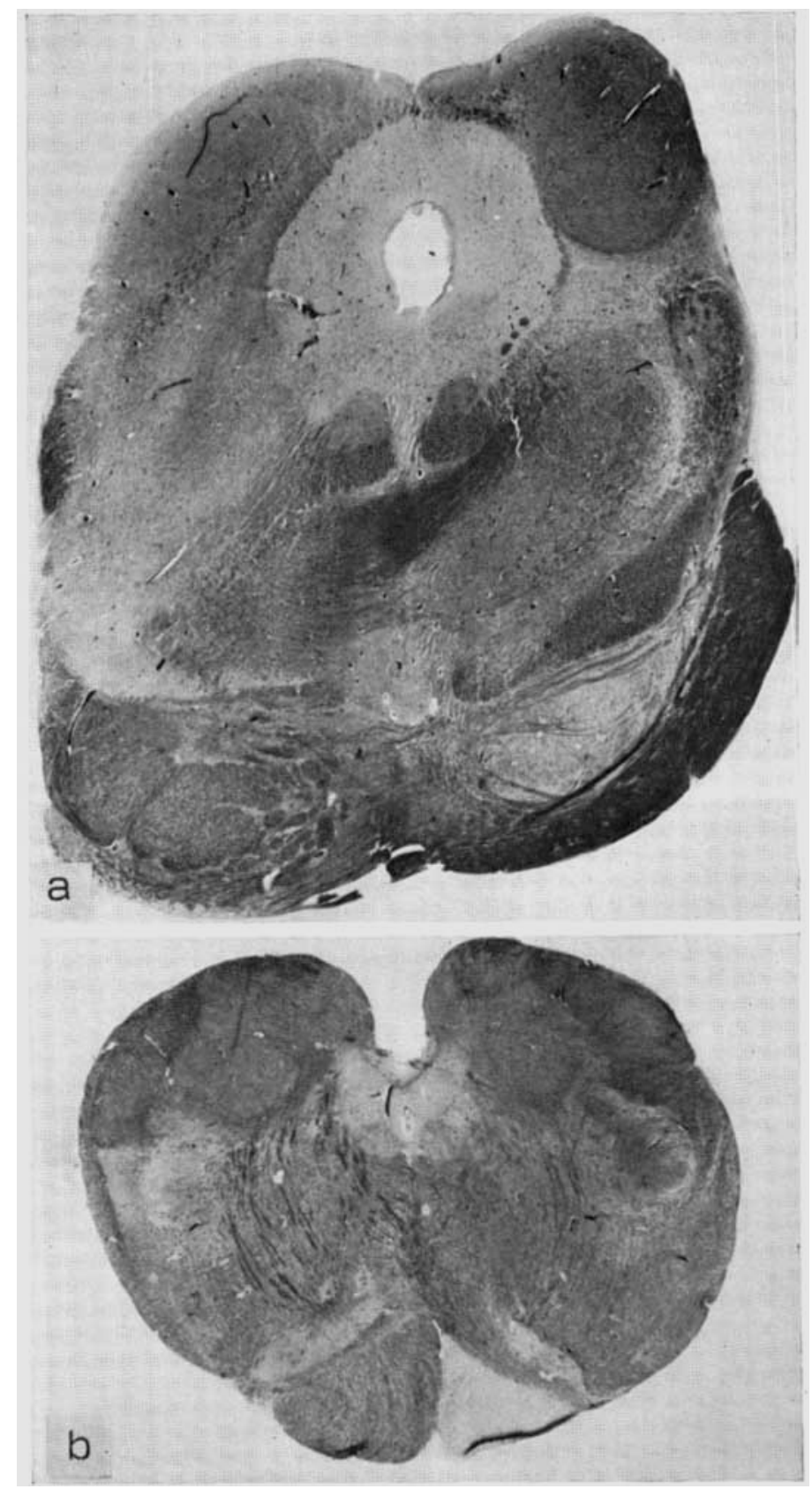

Fig. 36 Photomicrographs of brain of Monkey 4711. (a) Section through the midbrain at the superior cerebellar decussation. Degeneration in the tegmentum of the midbrain and in the left superior cerebellar peduncle is evident. Note degeneration in the corticospinal and corticopontine tracts on the right, $7 \times$. (b) Section through the medulla just in front of the obex showing degeneration of the spinal tract of $V$ on the left and of the pyramidal tract on the right, $7.5 \times$. 

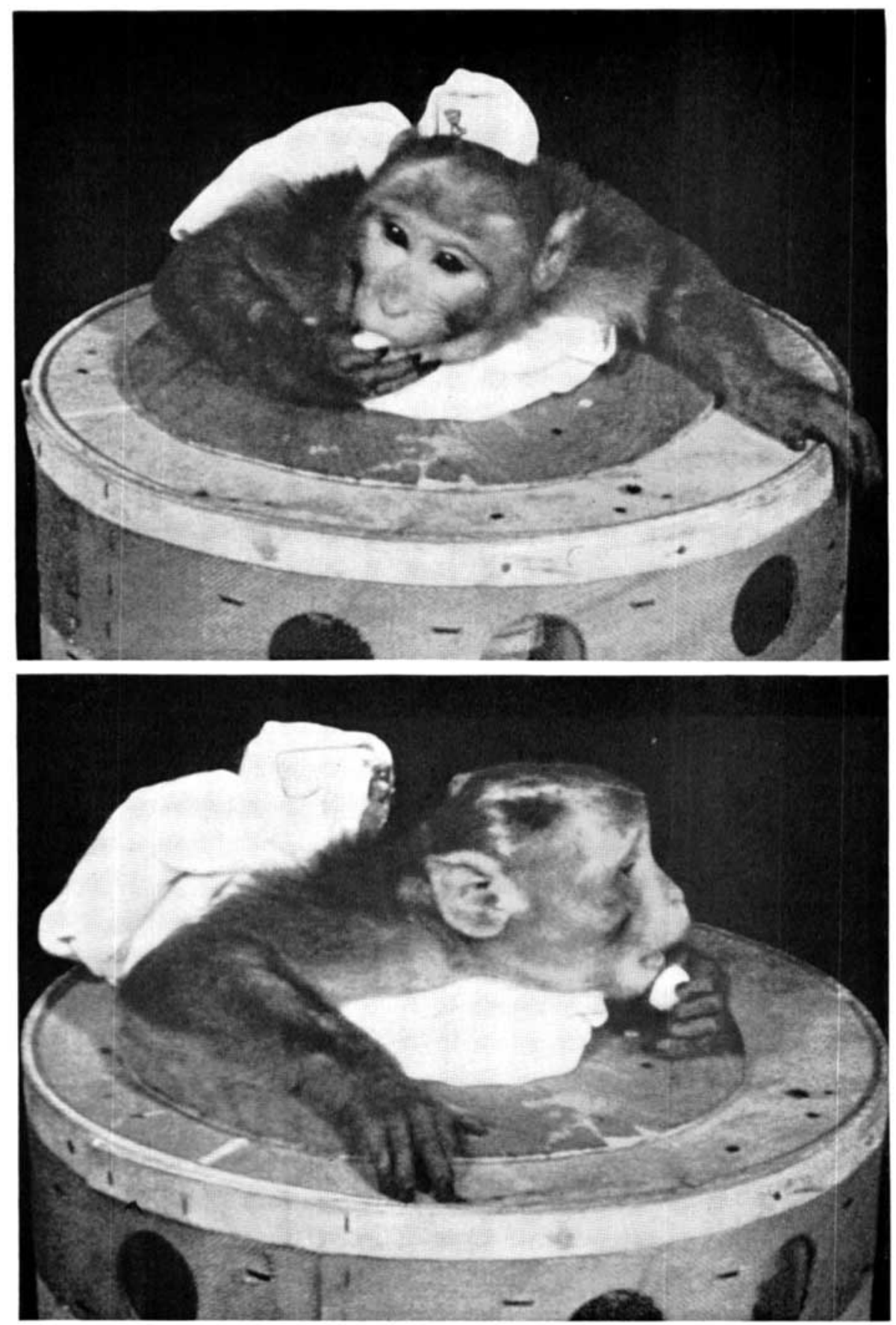

Fig. 37 Monkey 5646, February 20, 1964, showing recovered ability to use both hands in eating. 


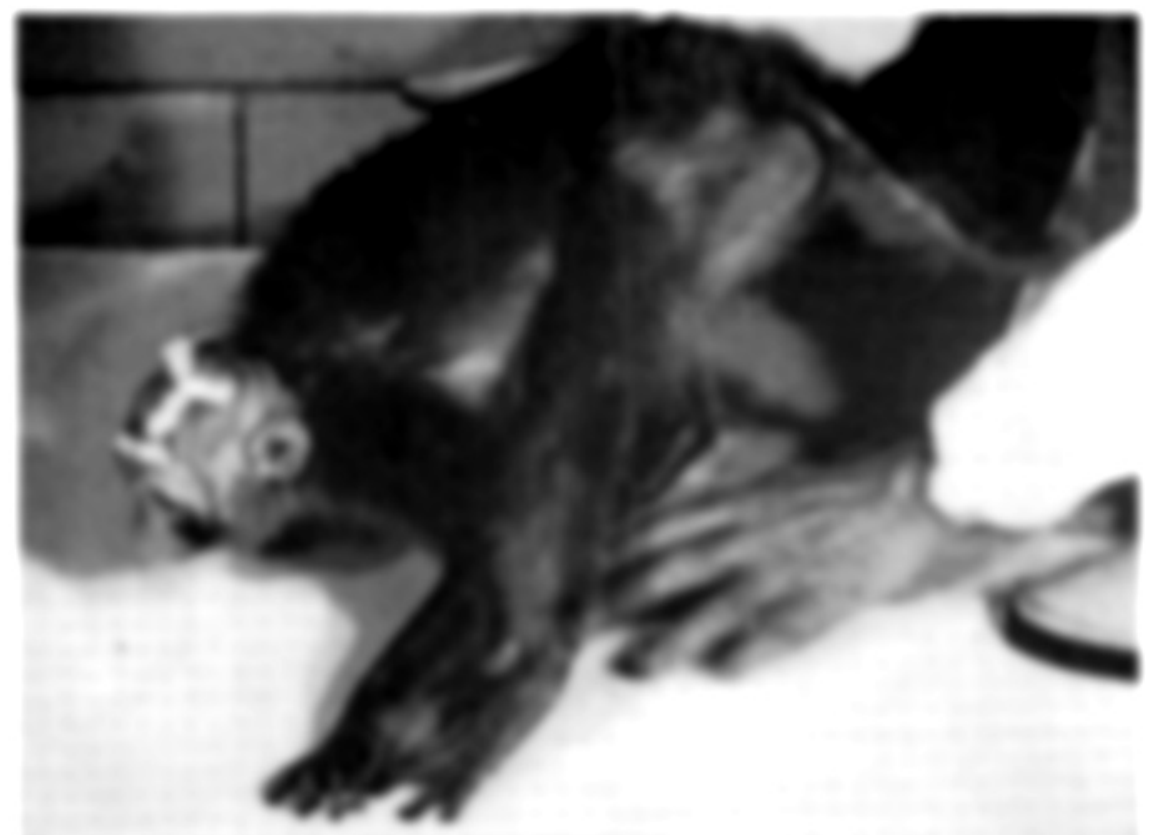

Fig. 38 Monkey 5646, Jume 9, 1964, showing hyperextension of the lower extremities.
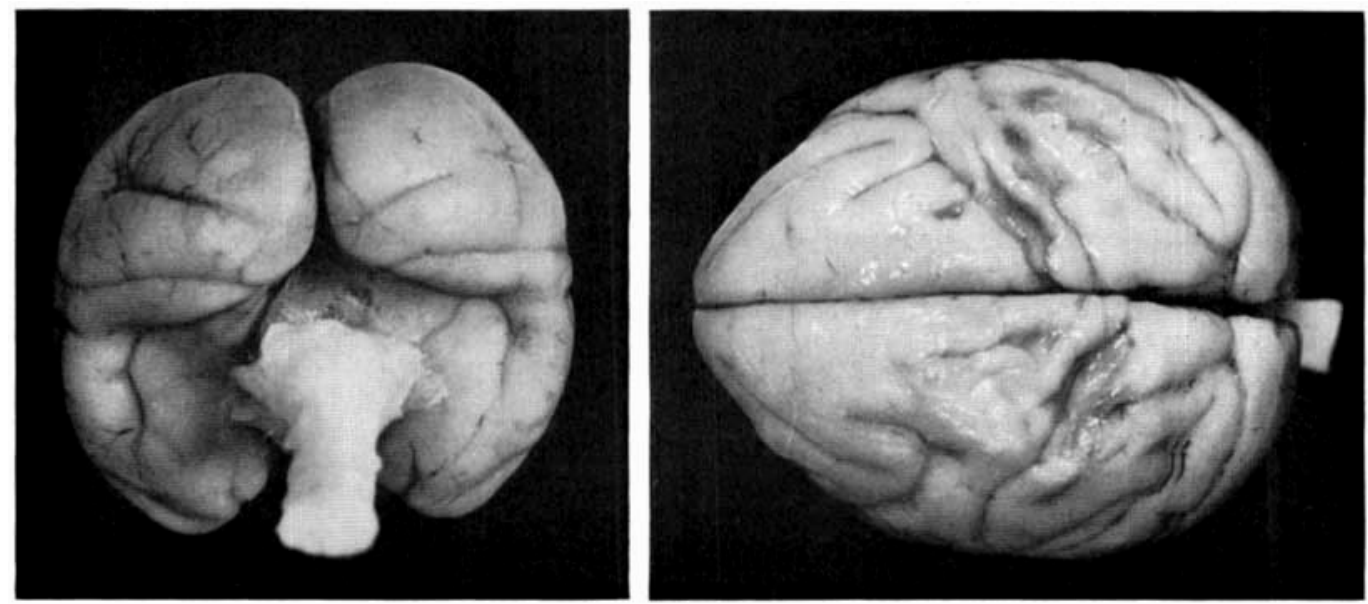

Fig. 39 Brain of Monkey 5646 postmortem.

alter it by cerebellar lesions of various sizes and locations, it was determined to test the results on a very limited group of especially selected children. The operations on the children were performed by Dr. Schneider.
All of the children operated upon, except two with dystonia musculorum deformans, had cerebral palsy and were subnormal mentally. One tested at I.Q. 24, another at I.Q. 54. From their postoperative performance it seems likely that they 
are actually somewhat more intelligent than these records imply but they are distinctly well below normal, probably an indication of the wide involvement of association areas (and supplementary motor areas) by the original lesions.

All of the children had intense hypertonicity. The muscular rigidity of the back in some cases was sufficient to produce severe lumbar lordosis. In the cerebral palsy children, there was an approach to an opisthotonic position, often with marked extension of the lower extremities and flexion of the upper extremities. The rigidity in children with dystonia musculorum deformans varied at different stages in the progression of the disease. An early rigidity in extension might be replaced later by a rigidity in flexion. In one of the children with cerebral palsy and in one with dystonia musculorum deformans, intense pain was the motivating factor for undertaking the operation.

In all cases the children had been examined by various physicians and their conditions regarded as hopeless. Drugs had failed to relieve the intense hypertonicity and the sedatives given to those children who suffered intense pain were gradually becoming less and less effective.

The cerebellar operation was tried with the hope that by obtaining a better balance between the discharges from the cerebral cortex and from the cerebellum an improved over-all condition might be obtained even though the brain lesions were increased. The cerebellar lesion consisted of a removal of the posterior vermis down to the ventricle and an extension of the lesion on out into the hemisphere bilaterally to destroy the cortex of the tonsil and of the immediately adjacent parts of the ansiform and gracile lobules, where they border the vermis laterally and the tonsil dorsally and laterally (fig. 52a, and $b$ ). Great care was taken to avoid injury to the dentate nucleus and to the small globose and emboliform nuclei, so that an action tremor should not appear.

GEORGE had a form of choreoathetosis as a result of erythroblastosis. After being studied extensively, he was admitted to University Hospital on September 8, 1958. At that time he was eight years old.

He was unable to speak, primarily because of spasms of the laryngeal muscles. He had also a considerable degree of mental retardation (his intelligence quotient was 51 ). He had been refused admission to the private schools for handicapped children to which his parents had applied. A physical examination showed a marked amount of spasticity, with more involvement of the right arm than of the left. He was unable to extend his fingers properly on the left. On the right, his hand was held in a flexed position at the wrist so that he could not use it effectively. At the slightest stimulus, both feet were thrown forward in marked extension. When he was stimulated, the left arm was frequently thrown upward above his head and the right arm extended, with the wrist flexed. His tongue tended to push up against his palate and his face became contorted in a facial grimace. The spasms were pronounced (fig. 40).

The skull x-rays, the results of the lumbar puncture, and pneumoencephalography were all normal. An electroencephalogram showed a diffusely abnormal pattern suggesting extensive bilateral brain damage.

Since it was impossible to control the child's symptoms medically, surgical therapy was discussed with the family. The parents were told some animal experimental work had given results which might be helpful. They were informed that the procedure involved a partial cerebellectomy and warned of various dangers associated with an attempt of this kind. Since they were unwilling to place George in an institution, and they could not care for him in his present state, they agreed to the operation.

Under local anesthesia, a complete suboccipital craniectomy was performed on September 18, 1958 and a region approximately $3 \times 3 \times 4 \mathrm{~cm}$ in volume was excised in the medial half of the

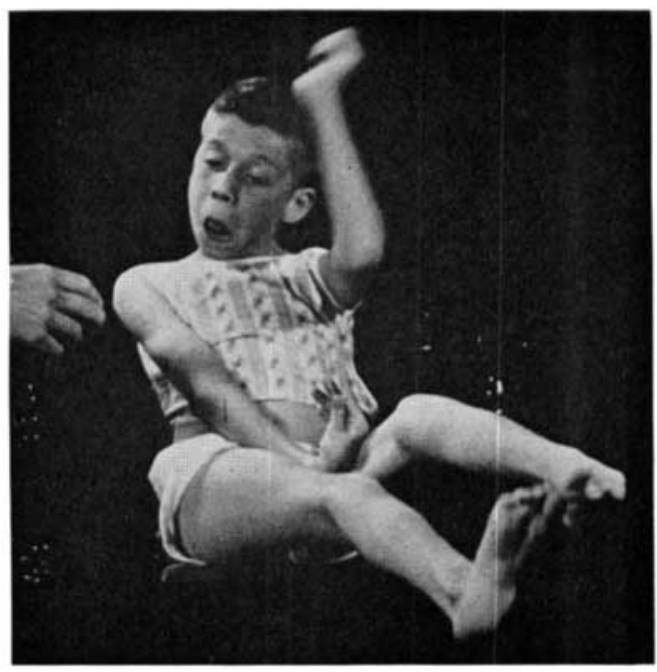

Fig. 40 George in terrific spasms resulting from the slightest stimulation. (Reprinted from the proceedings of the Second International Meeting of Neurobiologists, Amsterdam, 1959, by permission of the publishers, Elsevier Publishing Company - Schneider and Crosby, '60). 
right hemisphere of the posterior cerebellar lobe, being careful not to injure the dentate, globose, or emboliform nuclei. The tonsil was removed bilaterally and, also, the posterior part of the vermis (uvula, nodule, and pyramis) down to the ventricle but with attempted sparing of the rostral poles of the fastigial nuclei (fig. 41). During the operative procedure the patient suddenly became unresponsive without any alteration in the vital signs or in the anesthetic. No definite cause for this change in state of consciousness could be noted, so the wound was closed and the operation completed.

For a time, postoreratively, this child appeared to be considerably worse than before the procedure. He was lethargic. $\mathrm{He}$ had intermittent vomiting, although special care had been taken to avoid pressure on the respiratory and the vomiting centers in the floor of the fourth ventricle. $\mathrm{He}$ was able to move his head but constantly turned away from his examiner. He had smacking movements of the lips and associated movements of the tongue. At times he made grunting sounds accompanied by a cry. His pupils were equal and reacted to light. He tended to hold his head in extension but offered no resistance to flexion of the cervical spine. He had marked spasticity in all extremities, much worse than preoperatively. The only exception to this was seen in the left arm, which was somewhat looser than the right. He was unable to grip with the right hand and could not use the left hand and left fingers. There was a bilateral, coarse tremor, which was more marked on the right. The legs were drawn up close to the trunk with the thigh and the leg in flexion and there was evidence of scissoring. The athetoid movements had disappeared. He was discharged from the hospital on October 7, 1958.

On May 25, 1959, George returned as an outpatient for a check up. He showed marked im provement over his preoperative (and immediately postoperative) condition. He showed some athetoid movements but his spasticity was less. Some nine months postoperatively he had less involuntary movements of the head, a loss of the spasm of the lower extremities, and better control of his left arm. He could smile, so that his face lost the expressionless and sometimes distorted appearance which it had had before. He could swallow better than preoperatively and could take a glass of milk in his hand and drink from it - a feat he had never accomplished previously (fig. 42). His finger to nose test was reasonably well done. His lower extremities were becoming hypotonic and he had some voluntary flexion of them at the knees.

He continued to improve over the next three years. He attended a school with provision for teaching handicapped children and lived at home. He learned to write his name and some other short words and sentences. He did quite well in arithmetic. He played with trucks and other toys and developed a considerable interest in television. He talked in short sentences. He had a smiling, rosy face and seemed to get quite a bit

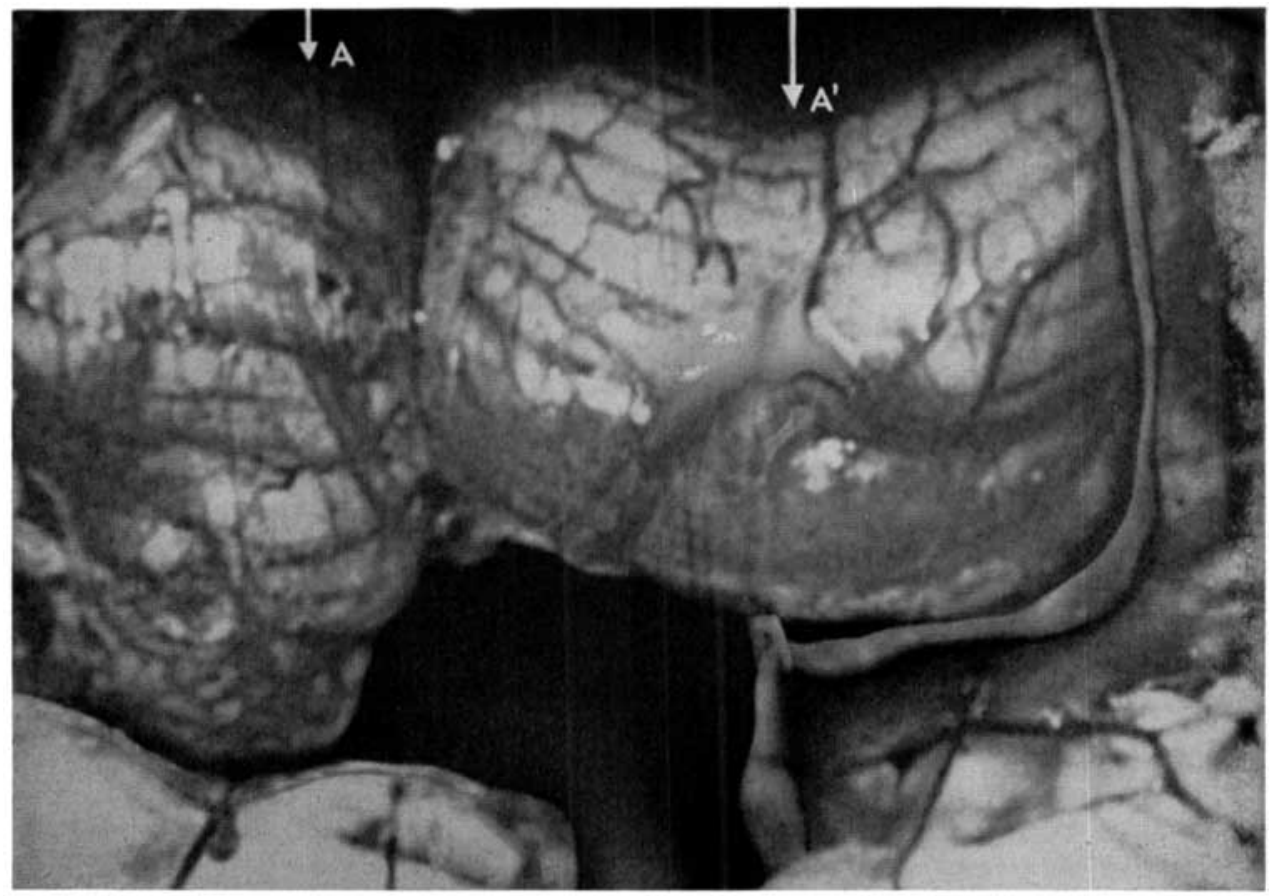

Fig. 41 Operative site after partial cerebellar excision. Comparison of the length of arrows A and $A^{\prime}$ suggests the extent of tissue removed. 

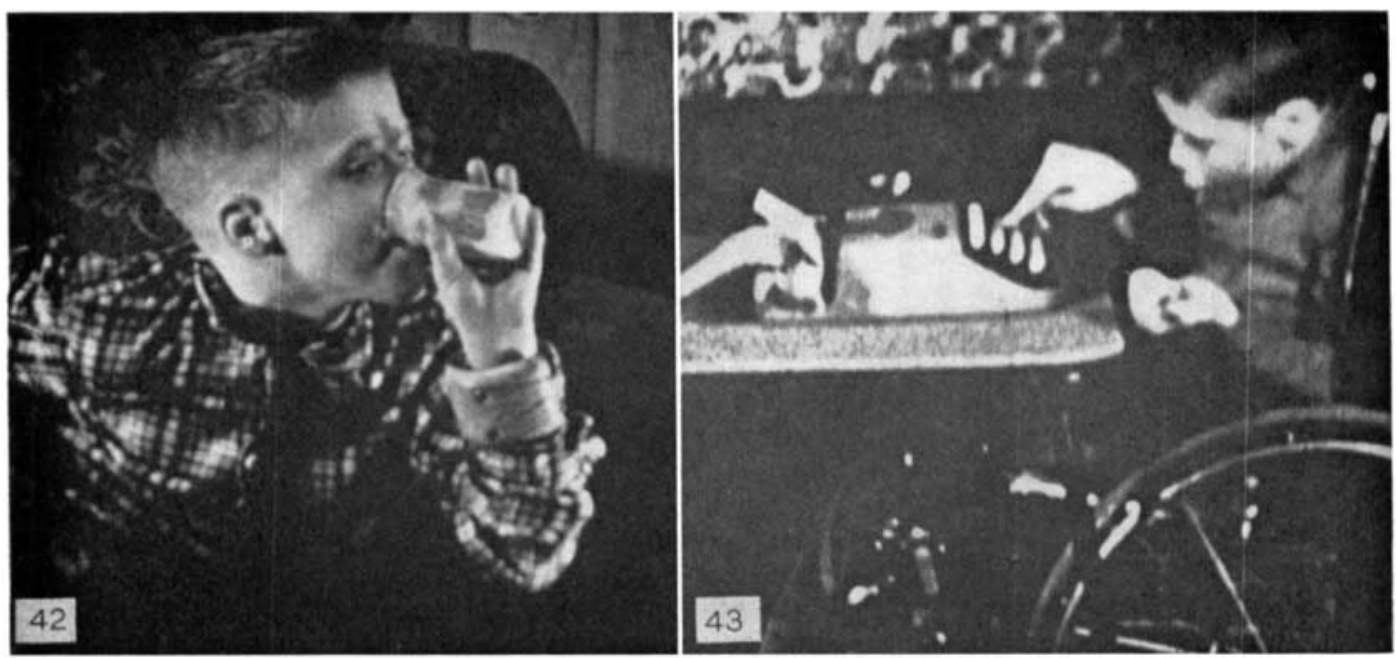

Fig. 42 George definitely improved postoperatively, on May 25, 1959. (Reprinted from the Proceedings of the Second International Meeting of Neurobiologists, Amsterdam, 1959, by permission of the publishers, Elsevier Publishing Company - Schneider and Crosby, '60.)

Fig. 43 George in the summex of 1962. (Reprinted by permission from the Editors of the Journal of the Michigan State Medical Society, 62: 175-204 - Schneider and Crosby, '63).

of fun out of living. By the end of the period, he was able to type simple words on an electric typewriter, using one finger and the "hunt and peck" method (fig. 43).

Five years postoperatively there was an increased hypotonia in the trunk muscles, suffciently great so that George tended to slump forward. His left hand moved more freely but he still had marked loss of control. His right hand had an athetoid posture. Nevertheless, the athetoid movements were gone. The spasm of the throat muscles had also disappeared and he could speak more readily than before. He was attending school and was able to color, to write simple sentences, and to read simple text. He was still better in arithmetic than in any other subject. Certainly his lack of mental capacity is in part responsible for his failure to acquire further skills.

At the present time he has shown some further improvement. He is attending school and is adding a little to his capabilities (fig. 44). His mental status makes it impossible to hope that he will ever be able to care for himself.

CHARLES, a ten year old, right-handed boy, was afflicted with dystonia musculorum deformans. At six years of age his right foot showed spasticity and a tendency to invert and plantar flex. Within a few months the right hand lost its dexterity and he switched to left handedness. Within a year it became impossible for the boy to sit up because of torsion spasms of the trunk and pelvis and he could barely walk without the aid of crutches. On attempts to move any limb, dystonic spasms abruptly intervened rendering the attempt fruitless. The head was tilted with the chin being forcibly pointed downward and toward the left side. The spine was frequently thrown into forcible curvature with a convexity of the kyphoscoliosis to the left. The involuntary movements were aggravated by any attempt to sit or stand and would decrease upon lying down. No intellectual deterioration occurred. The frequency and the severity of dystonic spasms, however, rapidly progressed to where the boy became bedridden with his limbs contorted and immobilized in odd postures with increasing rigidity and spasm. Voluntary movement was virtually impossible. Passive movement would initiate involuntary spasms throughout his entire body. Prolonged forced posturing of the extremity joints by unequal action of the antagonists led to stiffness of the joints, immobilization of them in an uncomfortable attitude, and increasing discomfort and pain. He could do nothing for himself except cry out in pain and required both paraldehyde and Demerol every four hours around the clock for any bit of short-lasting relief. Only accasionally in sleep would he be comfortable when the involuntary dystonic spasms ceased. It was so difficult for him to speak that he replied in staccato bursts of isolated words rather than full sentences.

Skull x-rays showed no apparent abnormality. His spinal fluid analysis was normal and pneumoencephalography revealed no abmormality. Electroencephalography both early and late in the course of the illness showed a diffusely abnormal tracing suggesting a degenerative process. With the hope of correcting the extreme muscular spasms and hypertonicity, a right cryothalamotomy was performed on September 30,1963 and a left cryothalamotomy was subsequently carried out on October 29, 1963. The forcefulness of dystonic spasms required that the operations be 


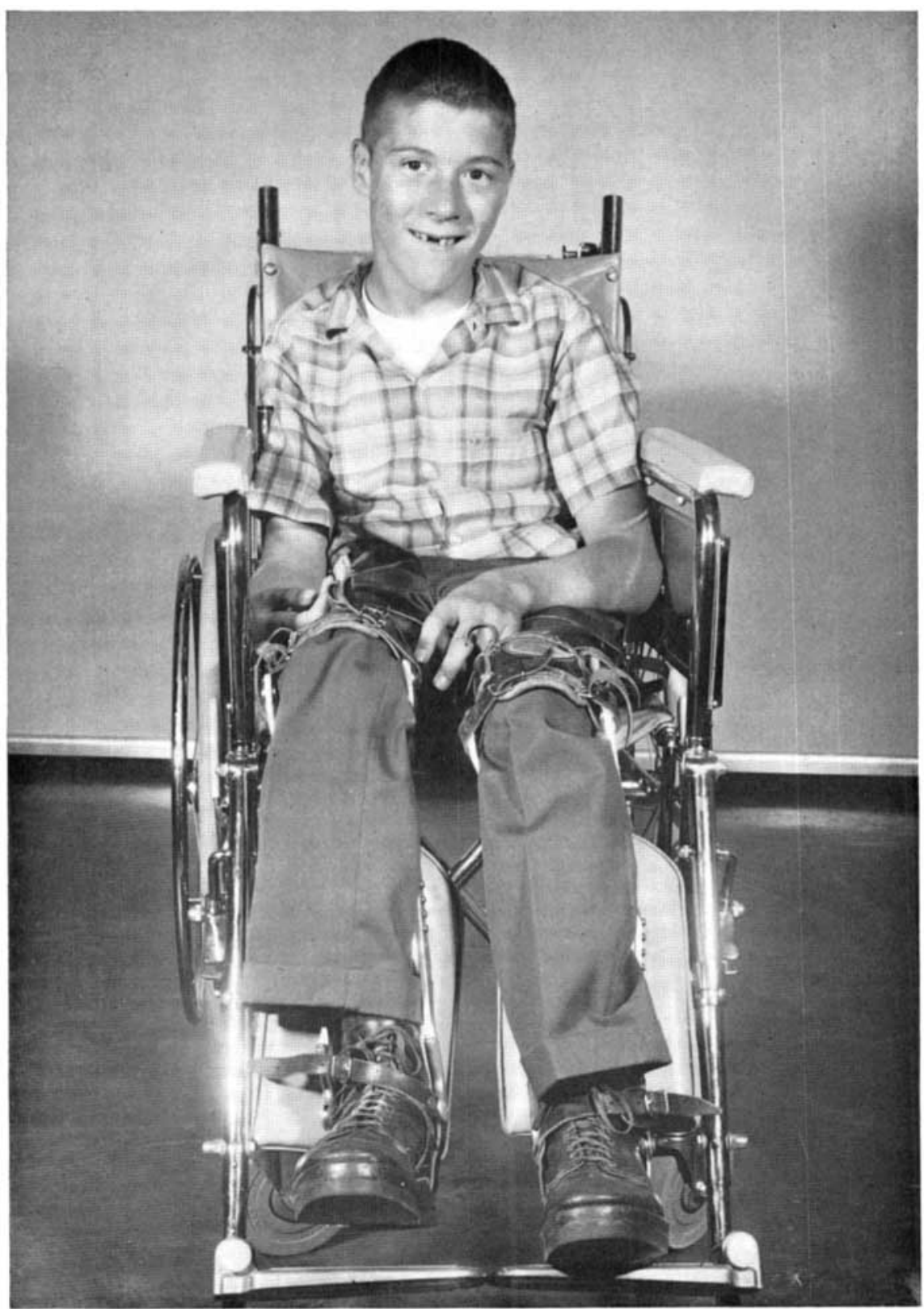

Fig. 44 George on September 20, 1964.

done under general anesthesia. In the right thalamus, three lesions of an esimated $8 \mathrm{~mm}$ in diameter were carefully made in the supposed location of the nucleus ventralis lateralis. No motor weakness resulted and no speech disturbance occurred. The dystonic spasms, rigidity, and posturing were only transiently slightly alleviated.
The boy's affliction was so terrible that some of the attending physicians believed, after failure of the cryothalamotomies, that to provide symptomatic relief even a tetraplegia with a total transection of the cervical cord would be preferable to the agony which this patient was suffering (fig. 45). Finally in the hope of re-establishing 


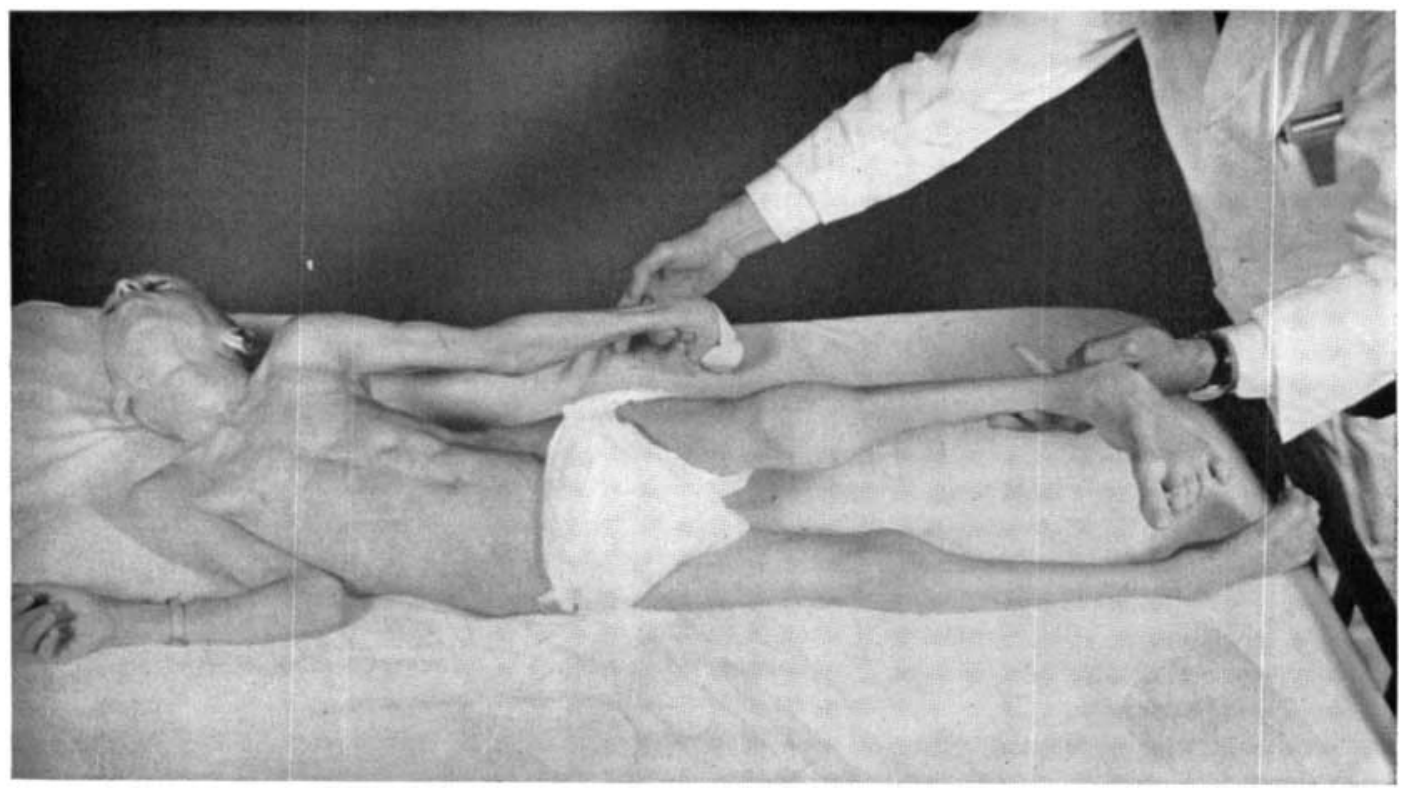

Fig. 45 Charles, November 2, 1963, suffering with agonizing spasms and intractable pain.

a new balance of tonicity and a relief of pain, a partial cerebellectomy was elected to be performed.

On November 13, 1963, a suboccipital craniectomy was carried out in a sitting position under general anesthesia. The posterior fossa was opened from one sigmoid sinus to the other. The dura was incised bilaterally exposing both cerebellar hemispheres and the vermis (fig. 46a). Beginning at the great horizontal fissure of the cerebellum superiorly, and working inferiorly, cerebellar cortex was removed from the vermis and from both the cerebellar hemispheres for a distance of $6 \mathrm{~cm}$ lateral to the midline and to a depth of approximately 1.5 to $2 \mathrm{~cm}$. In the region of the vermis, the lesion was deep enough to expose the ventricle and involve at least the more caudal part of the nucleus fastigii bilaterally (fig. $46 \mathrm{~b}$ ). The anesthetic was lightened in the hope of determining changes in tonicity on passive movement of the limbs. No noticeable decrease was observed in the rigidity of the left limbs but little if any rigidity appeared to be present in the right upper extremity. Inasmuch as the spasms and posturing were worse preoperatively on the left side than on the right, a little more of the posterior lobe of the left cerebellar hemisphere was removed to a depth of about 3 to $4 \mathrm{~cm}$, being careful to spare the dentate nucleus.

For nearly ten days following the operation the only noticeable change from the preoperative state was a worsening of the spasm and the rigidity. Then gradual improvement occurred. The spasms lessened in frequency and severity. Sedatives and narcotics were no longer needed for pain had disappeared. Within six weeks of the partial cerebellectomy the boy was able to feed himself with his right hand. The neck was no longer thrown back so far as preoperatively, and he tended to move his head from side to side without discomfort. He no longer showed the explosive, racing type of speech which he had had preoperatively, being now able to enunciate clearly.

The left arm which, preoperatively, had been held in rigid extension with the hand internally rotated and the fingers clenched so that the hand could not be drawn forward along the body, now showed considerable relaxation. It was possible to abduct the arm to nearly $130^{\circ}$ at the shoulder, to move the forearm under the arm, and to ma nipulate the one extremity without throwing all other extremities into spasm. The fingers did remain clenched and failed to open voluntarily. The least gain was noted in the left lower extremity. Both lower extremities could be flexed but with some difficulty and for only about $5^{\circ}$ at the knee; the toes on bath sides could be moved voluntarily though only slightly, Movement of the ankles was markedly limited by preoperative contractures but movement was possible at both hips, although more on the left than the right. The left lower extremity, which had been held in extension but jack-knifed at the waist level now could be drawn down toward the bed to approximately $30^{\circ}$ from complete extension.

By March 2, 1964 a further lessening of the disability was noted. The boy continued to be able to feed himself with his right hand. His left hand could be brought haltingly into flexion and curved forward onto the chest and there was some return of movement in the previously clenched left fingers. It was now possible for him to be seated upright (fig. 47) in a chair or bed whereas 


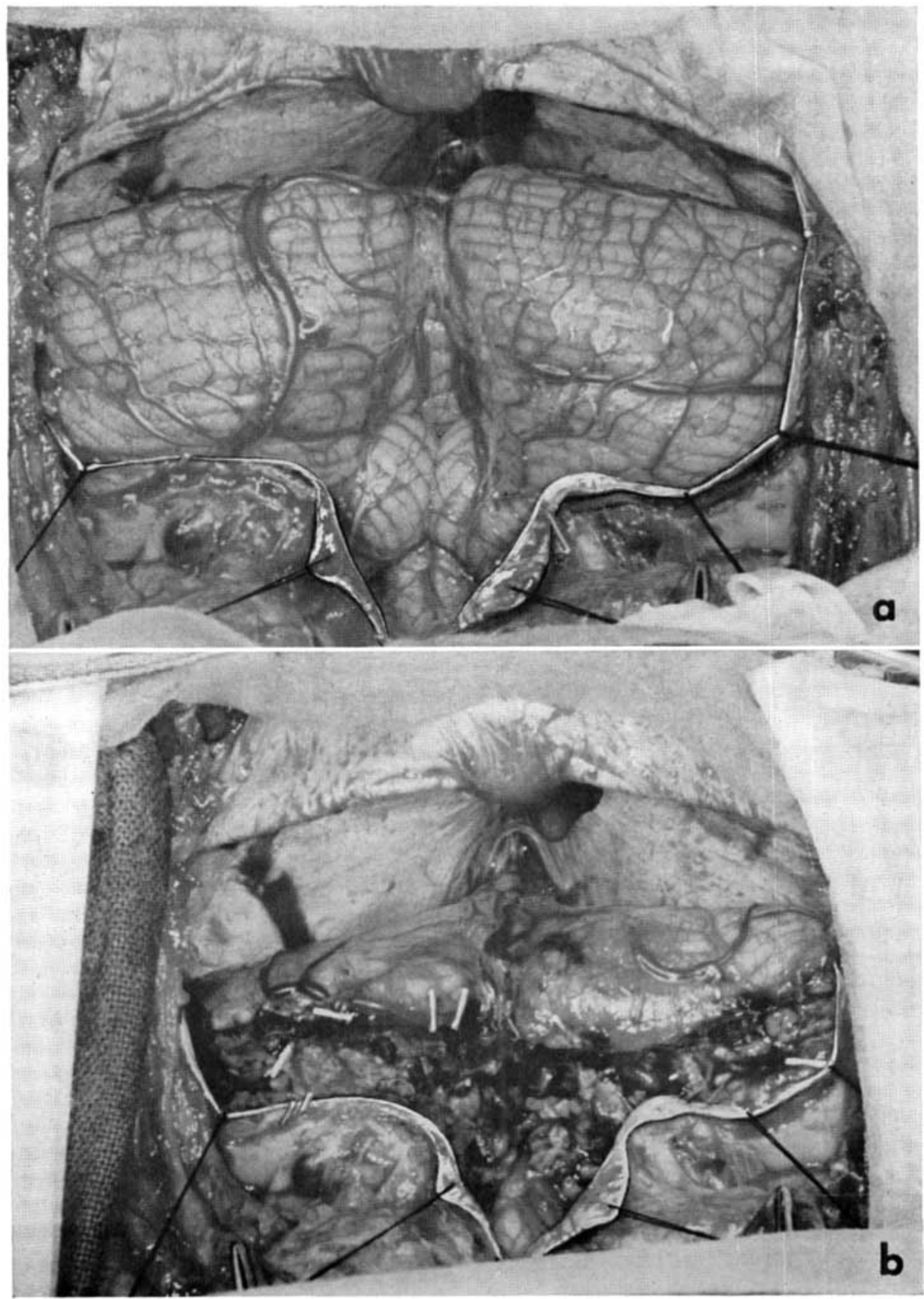

Fig. 46 (a) Operative field before surgical excision. (b) After surgical excision.

previously he was so rigid he could not be bent. In the lower extremities, he was able to move his toes slightly and, with little effort, he could flex his right leg at the knee. There was still less difficulty in speaking. Contractures at the wrist and the ankle joints still limited greatly the movement of the less hypertonic muscles of the extremities. An X-ray demonstrated a dislocation of the left hip which, in the interim, had formed a false joint. 


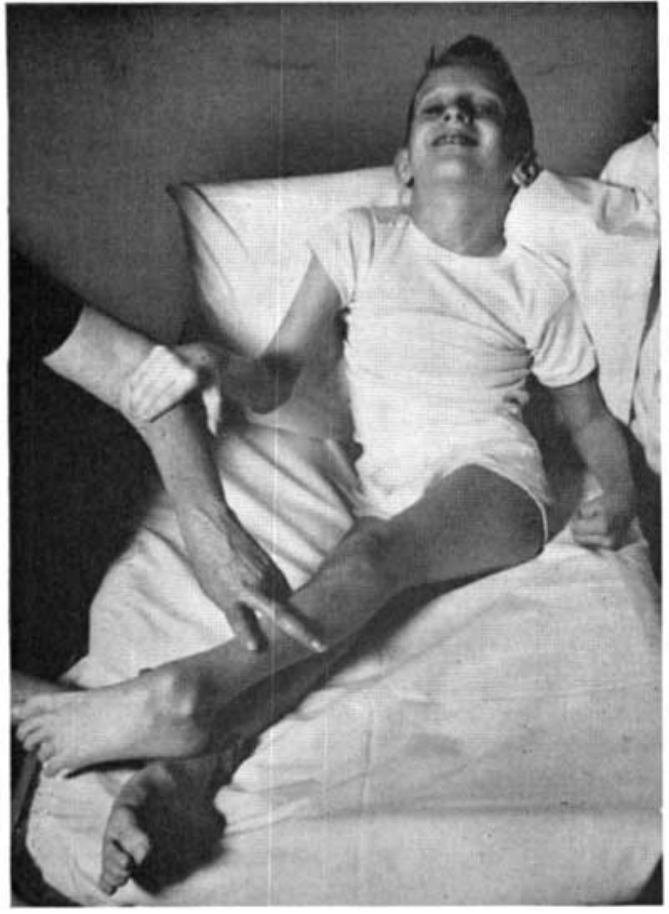

Fig. 47 Charles being examined postoperatively in February of 1964 . Note the sitting posture and freedom from pain.

By April 7, 1964, the right hand had sufficiently loosened up to permit Charles to color pictures in a book. His left arm continued to show a diminution of stiffness, but it was still noticeably disabled, and it took considerable effort for him to bring his arm up over his trunk. Some involuntary movements of the arm were still present. The boy could actually assume a sitting position although the hips remained somewhat rigid. The right leg could be actively moved. He could flex and extend it on the thigh quite readily (fig, 48) Marked flexion deformities of both ankles persisted. The entire left lower extremity remained somewhat rigid and extended upward. Now he could smile for he had no pain. He spoke in well modulated sentences.

Examination on September 9, 1964 unfortunately showed some progression of his illness. The tone had increased in the right lower extremity so that the limb was more or less fixed in flexion at the hip but he could flex the leg considerably. The left lower extremity showed fixation in extension and very little flexion at the knee was possible. He still could be seated and maintained in a sitting position. Fortunately good function of the right hand continued and there was no further stiffening of the neck and no pain. The left hand and arm could still be swung over the chest but hesitantly, and there was no functional movement of the left hand and fingers.

It was decided not to proceed with further cerebellar excision. In the hope of providing some relief from the rigid lower extremities, a release of the flexor and the adductor muscles of the right hip capsule was carried out on August 28, 1964 , and a release of the adductors and the flexor muscles of the left hip on September 10, 1964.

BRENDA is now a 12 year old, right-handed girl. At six years of age she developed progressive difficulty in walking and athetoid movements, particularly on the left side of her body. She was first seen by the neurosurgeons on April 4, 1961, at which time the most striking signs were a markedly increased tone in the back, the abdomen, the thighs, and the legs. The muscular

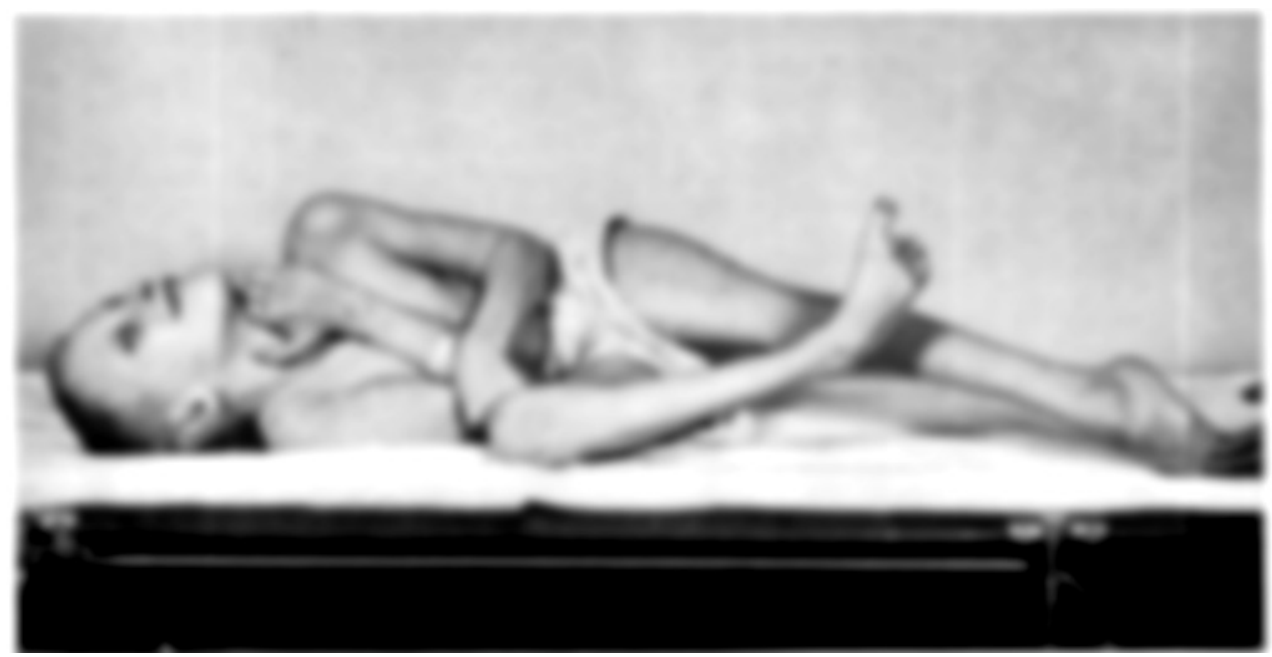

Fig. 48 Charles, June 9, 1964, showing ability to flex the left upper and right lower extremities. 
rigidity was so great that she had a severe lumbar lordosis, which bent her trunk toward the right side. The abdominal musculature was remarkedly tensed. Both feet were kept in an equinus position with the toes flexed. Her gait was very unsteady with the left leg kicked far out in front of her as the foot was picked up. She walked on inverted tiptoes. Because she was still ambulatory, surgical therapy was deferred. By September 6, 1961, the child had a marked progression of her symptoms, for she now had marked flexion deformities of the left foot and the left knee (fig. 49). Her psychological testing showed that she was functioning at the upper end of a dull-average range of intelligence.

On June 25, 1962, the child had a right stereotaxic thalamotomy in which two lesions were made by the Bertrand stereotaxic snare technique. At the time of her discharge from the University Medical Center, on July 19, 1962, she had good strength in, and control of, the right leg but moderate difficulty in bringing the left leg forward because of the equinovarus deformity. She had marked relief of her symptoms and was able to walk about and feed herself.

In November, 1962, she began having abnormal movements. On January 8, 1963, she had a left cryogenic thalamotomy, with the insertion of two lesions. Postoperatively, she was markedly obtunded for a time. She had a minimal right hemiparesis and a moderate aphasia, which grad ually improved. Her abnormal movements were greatly diminished on the right and slowed on the left side (fig. 50).

On May 24, 1964, Brenda was readmitted to University Hospital. At that time she had some opisthotonic posturing. Both knees were flexed with a mild degree of contracture. The left knee was worse than the right and the left heel actually rested against the buttocks. Both ankles were held in flexion and in inversion. The trunk had a lordotic posture with the neck extended (fig. 51).

On June 4, 1964, the patient had a partial cerebellectorny in which the posterior vermis down to the ventricle and the cortex of the tonsils and of the immediately adjoining parts of the gracile and ansiform lobules were excised (fig. $52 \mathrm{a}, \mathrm{b})$. For the first postoperative week, as is usual in this type of operation (p. 48), the patient was much worse than before the removal of the cerebellar area. She was more opisthotonic, with her head extending backward within one foot of the buttocks. The trunk and the lower extremities then exhibited an increased tonus. The legs, moreover, showed some extension but the arms were somewhat more relaxed and could be used a little better than before the procedure.

Within a week, the child began to improve markedly. There was an amazingly rapid diminution of the hypertonicity so that, by the sixth day after operation, she could sit upright in a chair. At the time of her discharge from the hospital, on July 20,1964, she was able to walk with support and could move about fairly well. Her greatest problem at present, aside from the contraction and inversion of the left extremity, is a loss of stability of the trunk, which keeps her from standing and walking without help (fig. 53).
Her mentality, although somewhat blunted, is still fairly good. It is expected that she will continue to improve.

\section{DISCUSSION}

Among the various results of this long series of experiments which might be discussed, two topics are primarily considered.

They are: (a) the functional differences in the roles of the motor and the supplementary motor (or extrapyramidal) cortical areas for the elaboration of voluntary and automatic associated responses; and (b) the regulation of tonus at higher brain levels.

Various pertinent references to the literature are included in the small type, for the benefit of those who wish to document certain points. In reading the discussion this small type may be omitted without loss of continuity.

\section{Functions of motor and supplementary motor cortices}

\section{A. Motor cortex}

\section{Pattern on motor cortex}

The highly specific motor pattern demonstrable along the primate central fissure has been described for subhuman primates by various experimenters and for man by the neurosurgeons. It has been documented repeatedly in the monkeys employed in the present experiments, having been used to determine the size and the extent of the contemplated lesions.

Eye deviations in different planes have been obtained from stimulation of the frontal cortex rostral to area 4. Not all observers have agreed as to a possible pattern arrangement based on the direction of eye deviations. Some of the differences in results depend on the kind and the depth of anesthesia, if an anesthetic were employed. The evidence for a duplicate or mirror image pattern in the frontal eye fields, with recognition of a primary and a second motor area for eye deviations, is based on the work of Crosby, Yoss and Henderson ('52) and Brucher ('64) for monkeys and of Lemmen, Davis, and Radnor ("59) for man. The two papers quoted for monkeys agree quite well except that Brucher ('64) obtained no divergence of the eyes from stimulation of the frontal eye fields and he did get associated move- 

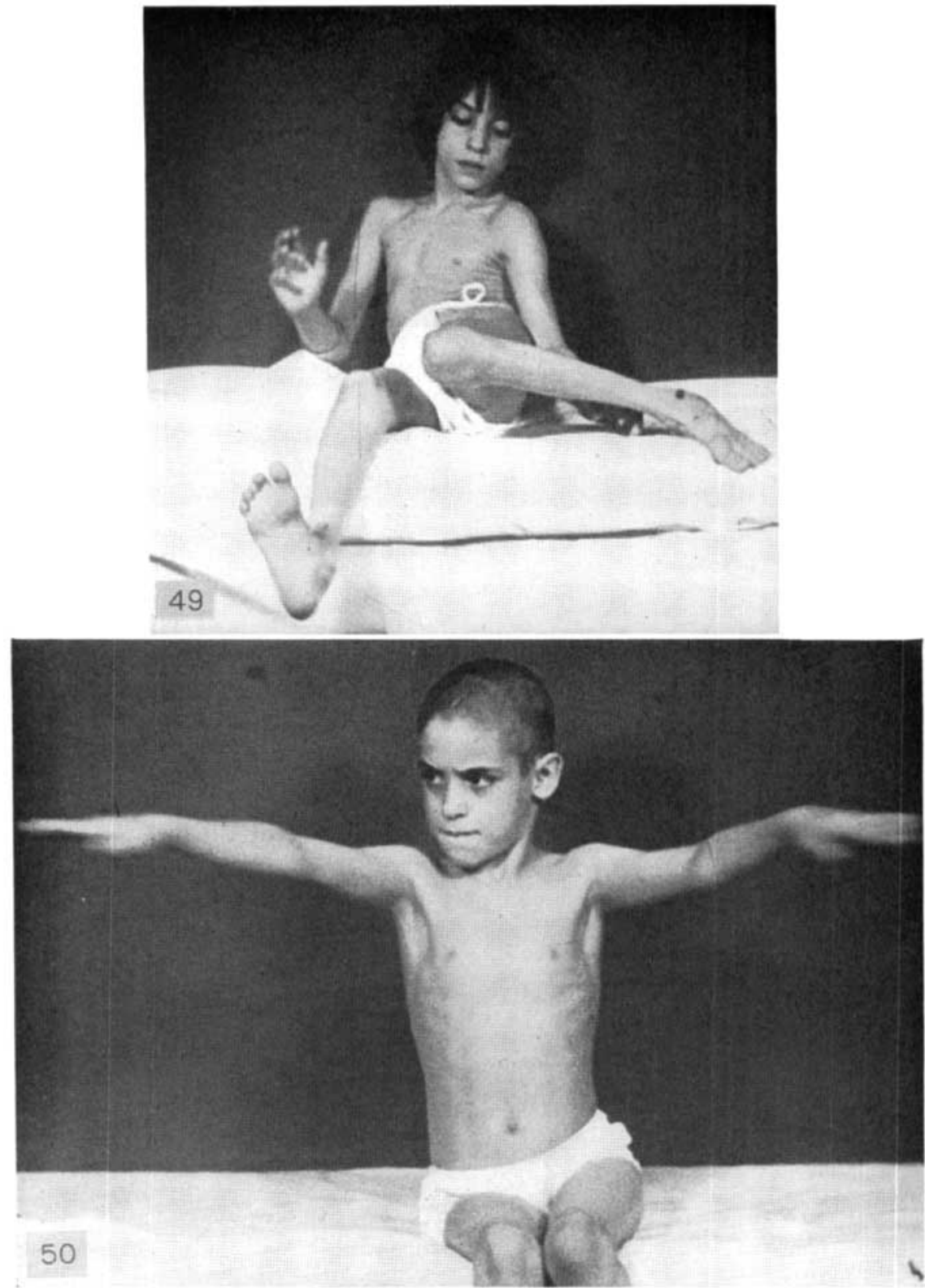

Fig. 49 Brenda, September 6, 1961, exhibiting dystonic posture.

Fig. 50 Brenda three weeks after the thalamotomy.

For acknowledgment for the use of these figures, see figure 52, page 54.

ments such as turning the head that were not reported in the earlier paper which dealt only with extraocular movements. Convergence and divergence of the eyes have been reported from excitation of the frontal eye fields by Jampel ('60). The present study illustrates forced deviations of the eyes from frontal lobe involvements.

The patterns of movements elicitable on stimulation of areas along the central fissure have been described by Foerster ('36), Woolsey ('38, '52, '58), Scarff ('40), Rasmussen and Penfield ('47), Penfield and Rasmussen ('50), Woolsey et al. 


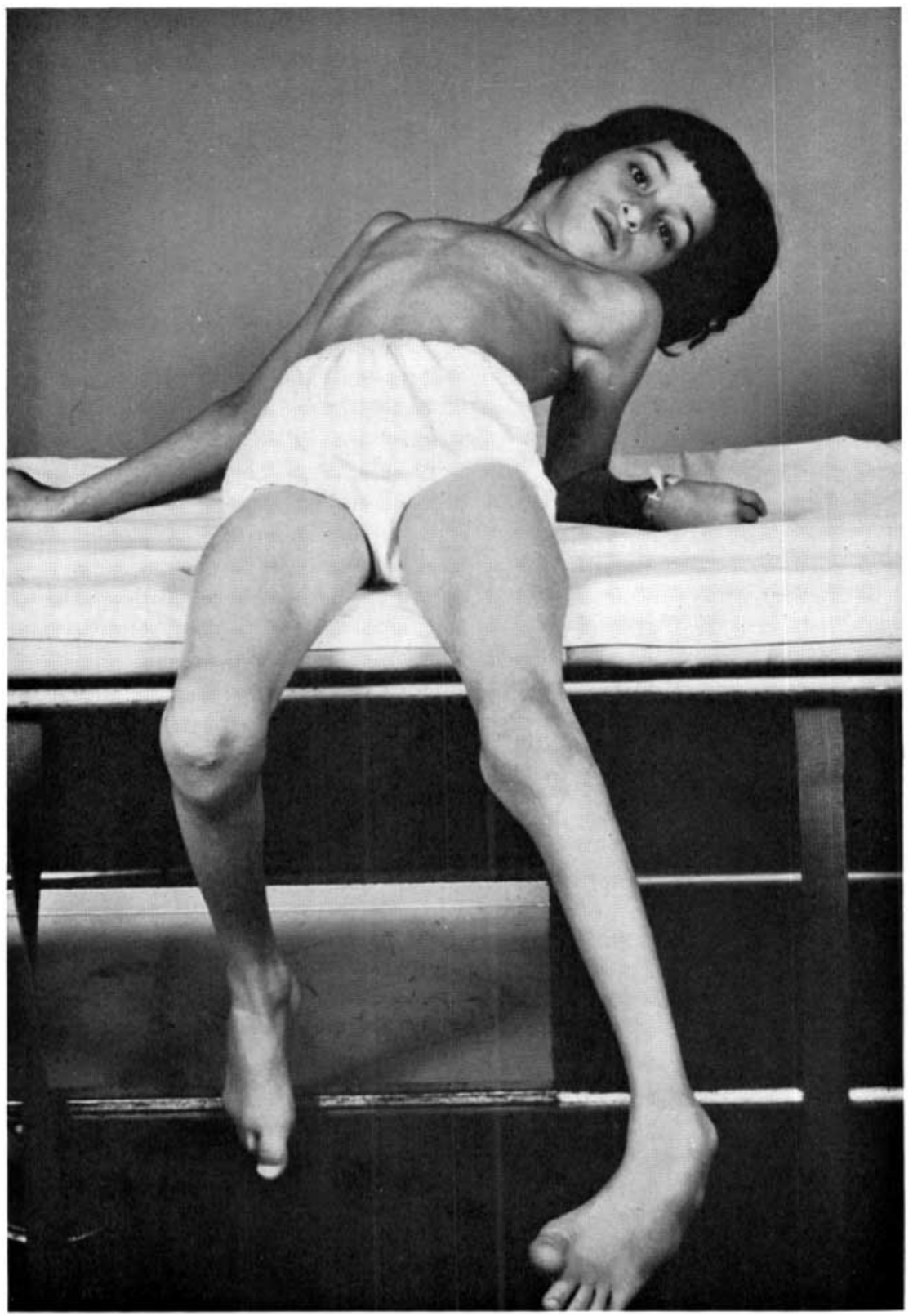

Fig. 51 Brenda in recurrent dystonic posture preceding cerebellectomy on May 29, 1964. For acknowledgment for the use of this figure, see figure 52, page 54 .

('49, '50, '52, '60), and many others. Eye deviations can be obtained from stimulation of certain portions of area 4 (chiefly the hand and the face regions), as various observers (Sherrington, 1893; Penfield and Rasmussen, '50; Crosby, Yoss and Henderson, '52; Crosby, '53a) have found. The patterns on the primary motor eye fields have been reported upon by Mott and Schaefer (1890), Grünbaum and Sherrington ('01), Vogt and Vogt ('07), Foerster ('31), Smith ('36, '49), Penfield and Rasmussen ('50), Crosby, Yoss, and Henderson ('52), Crosby ('53a), Lemmen, Davis and 

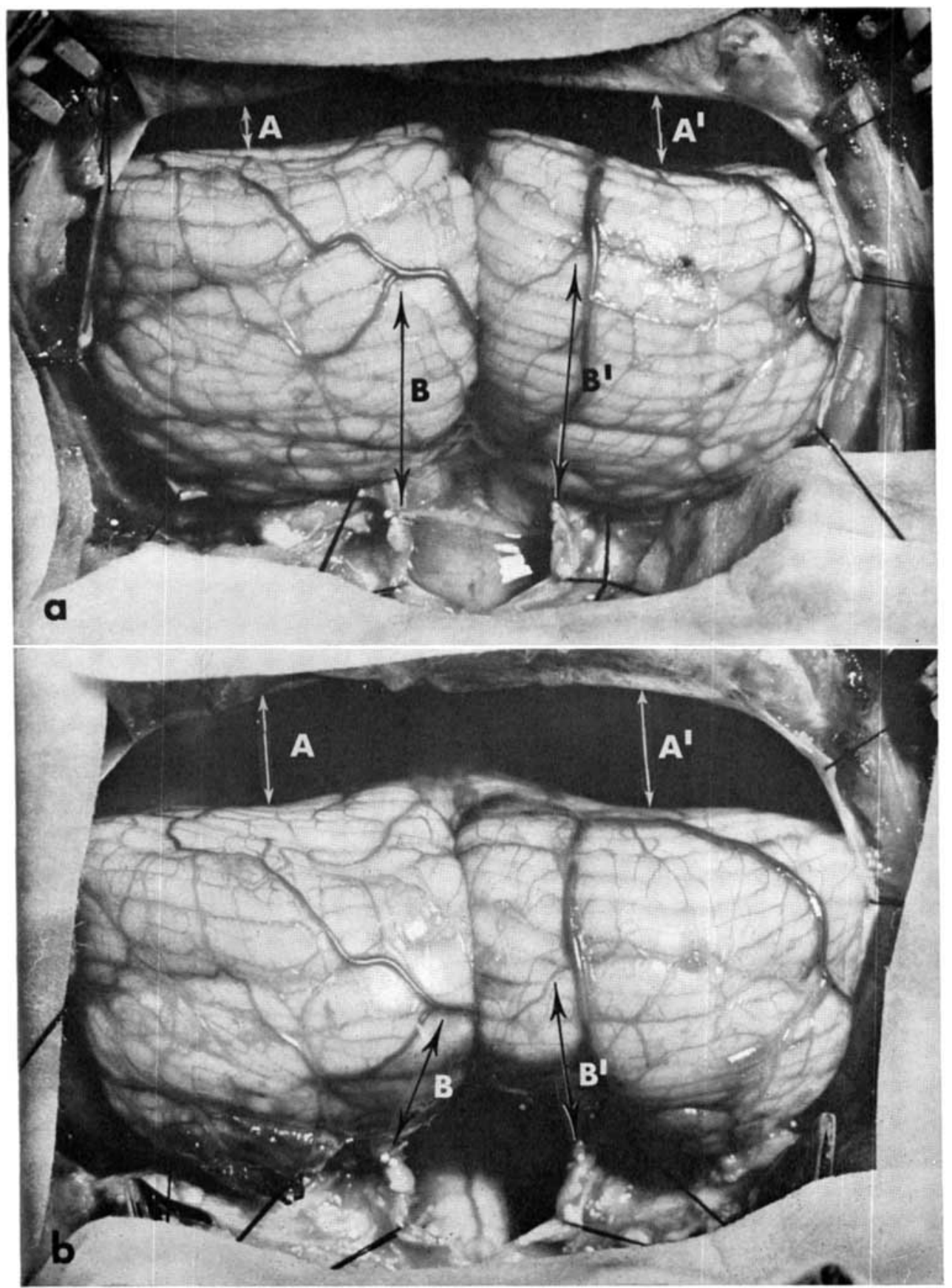

Fig. 52 (a) Before surgical excision of cerebellum. (b) Following surgical excision. The amount of tissue removed may be appreciated by comparing arrows $A$ and $A^{\prime}$ and $\mathbf{B}$ and $\mathbf{B}^{\prime}$ between views. (Figures 49 to 52 are reprinted from the Journal of the Michigan State Medical Society, 62: 175204 - Schneider and Crosby, 1963). 


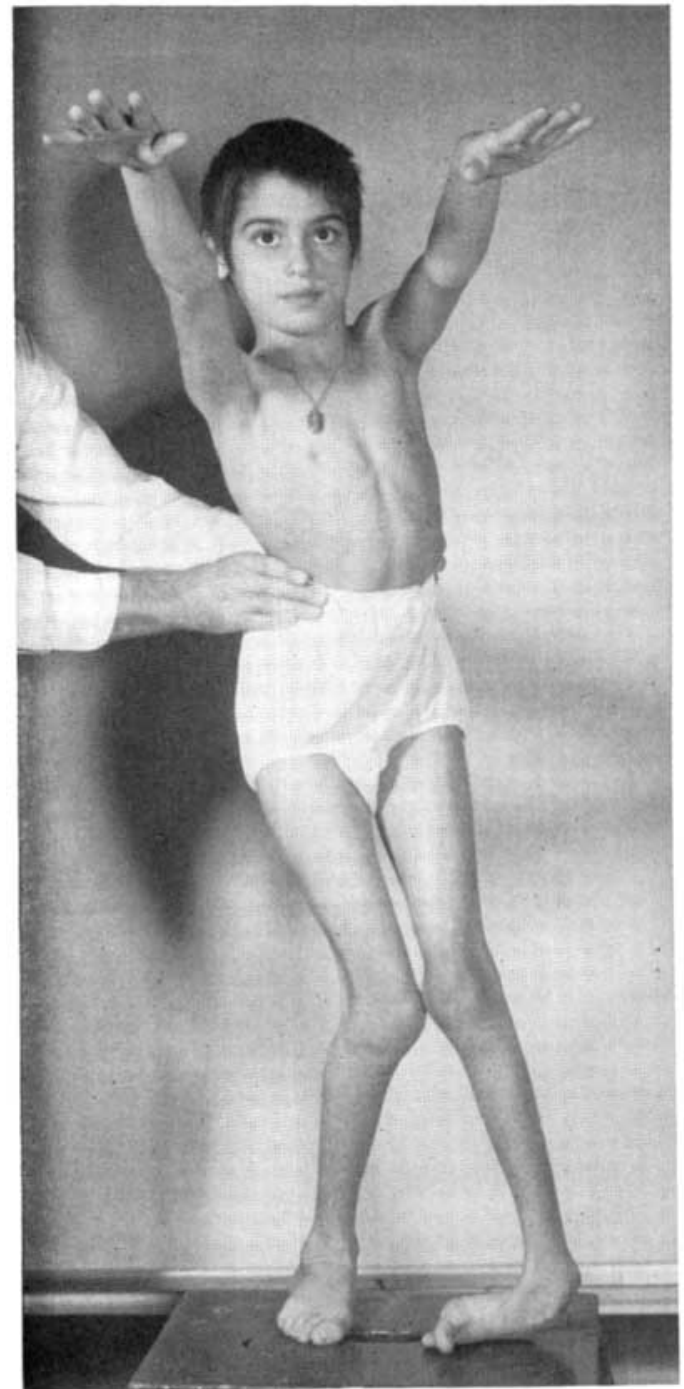

Fig. 53 Brenda in September of 1964.

Radnor ('59), Crosby, Humphrey and Showers ('59), Brucher ('64) and others working in this field.

\section{Discharge paths from motor cortex}

In addition to contributions to commissural and association bundles, the areas along the central fissure give rise to corticostriatal and corticotegmental fibers (p. $59)$ and to pyramidal tract fibers. The pyramidal tract contains not only the well known corticospinal and corticobulbar components but it has also fascicles of various origins and terminations. After rather wide-spread cortical injuries, including destruction of area 4, many pyramidal tract fibers survived (Lassek, '54). Along with fibers from paracentral and precentral gyri of the same side and, to a small extent, from the other side (figs. 54,55 ), there are fasicles in the pyramidal system from the postcentral gyrus, the parietal areas 5 and 7 , and perhaps (Walberg and Brodal, '53) from the temporal and the occipital cortices. The main bundle is joined in the internal capsule by corticobulbar fibers from the frontal eyefields. Fibers are said to ascend from cord levels in the corticospinal tract (Brodal and Walberg, '52, for the cat). The impulses they carry are thought, possibly, to have an inhibitory effect over corticospinal discharges (Walberg and Brodal, '53). The course of the pyramidal tract (figs. 54, 55 ) through the internal capsule and the brain stem and its terminations therein, its partial decussation at lower medulla and upper cord levels, and the termination of some of its fibers (Hoff and Hoff, '34; Kuypers, '60a, b) directly, and through intercalated neurons, on alpha neurons, and possibly on gamma neurons, are too well known to require further documentation. (For cat, Nyberg-Hansen and Brodal, 63.)

Physiological and anatomical findings indicate that some of the pyramidal fibers (presumably those from somesthetic and parietal cortices) distribute to various afferent nuclei - such as the nuclei of the posterior columns, the sensory trigeminal complex, and the intermediate gray, the gray at the base of the posterior horn, and the dorsal nucleus of Clarke in the spinal cord (Hoff and Hoff, '34; Chambers and Liu, '57, '58). Presumably such fibers are regulatory, and perhaps at times inhibitory, over the discharges from these afferent centers.

Peele ("44) suggested a sensory regulatory action of the pyramidal system furthered by fascicles in that system from postcentral and parietal regions. Scherrer and Hernandés-Peón ('55) stated that stimulation of the sensory cortex inhibits the passage of impulses over synapses in nucleus gracilis. They believed, however, as Hagbarth and Kerr ('54), that such inhibitory impulses were relayed to the 

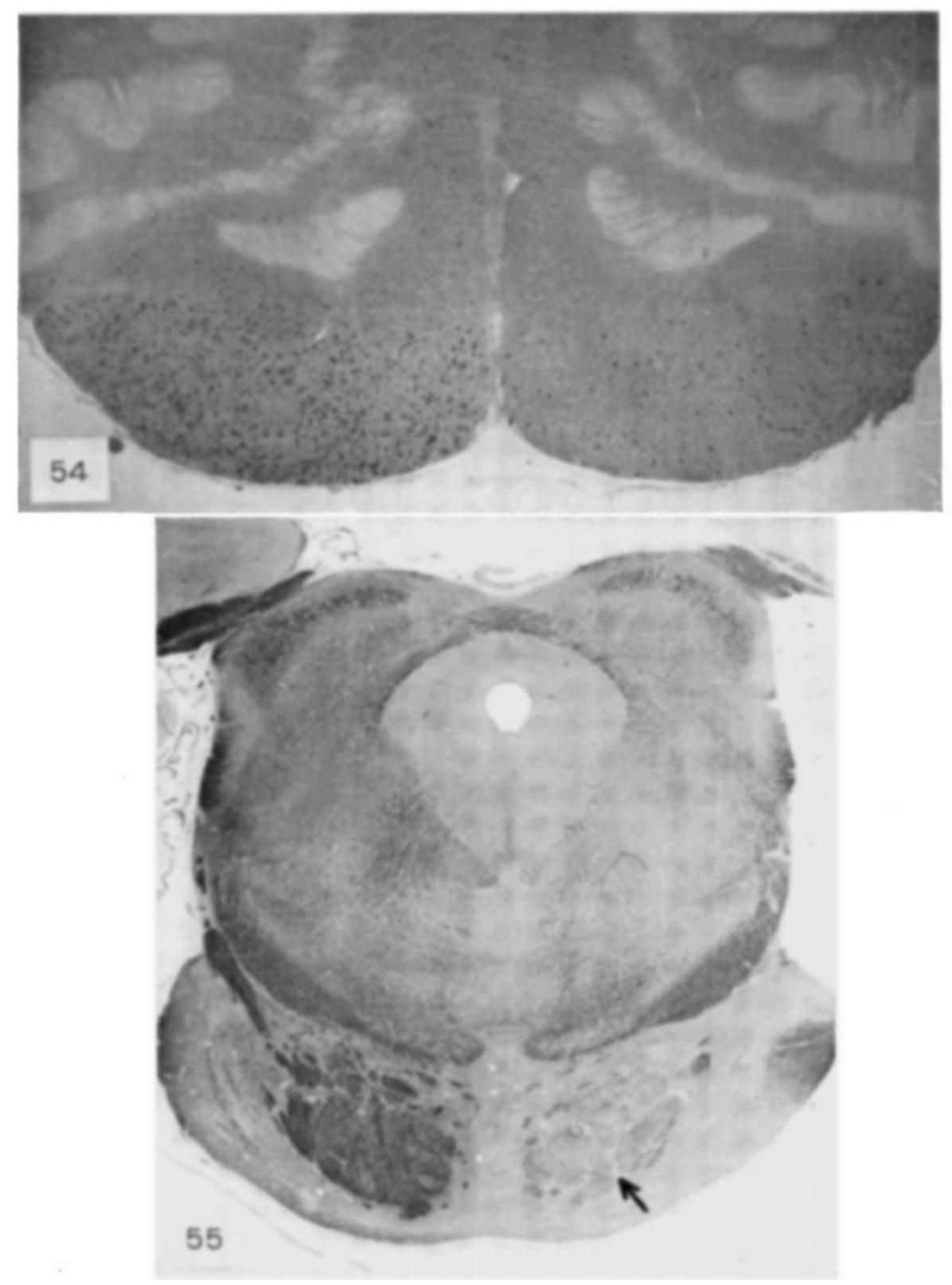

Fig. 54 Marchi degeneration in the homolateral pyramid and lesser degeneration in the contralateral pyramid following destruction of area 4 . It indicates the presence of homolateral and contralateral fibers in the system above the motor decussation, $21 x$.

Fig. 55 Weil preparation at midbrain-pontine transition showing a major degeneration in the pyramidal and corticopontine systems homolateral to an extensive cortical excision (arrow) and a very minor degeneration in the bundle contralateral to the cortical lesion, $6.7 \times$. 
sensory nucleus through the reticular formation.

The tonic activity (Adrian and Moruzzi, '39) or continuous discharge of the pyramidal system - reinforcing, facilitating, and, presumably at times, inhibiting the movements set up at various brain and spinal cord levels - was regarded by Tower ('49, see also Tower and Hines, '35; Tower, '40) as showing a functional organization of the pyramidal tract "in time." During sleep this tonic activity is greatly diminished. Tower's concept of a functional organization of the pyramidal tract "in space" refers to the pattern representation on the motor cortex, the pyramidal tract, and the lower motor nuclei of the brain stem. The present writers regard these patterns as determined by the peripheral distribution of the axons of those motor neurons constituting the motor pools on which, directly or indirectly, the pyramidal tract fibers end.

Along with fibers from the paracentral and precentral gyri (Holmes and May, '09; Hines, '37; Lassek, '42; Tower, '49; Glees et al., '51; and many others), there are fascicles from the postcentral (Peele, '42; Woolsey and Chang, '48), the parietal areas 5 and 7 (Peele, '42; Woolsey and Chang, '48), and $4 \mathrm{~s}$ (Hines, '42, '43), and, at least in the cat, from temporal and occipital cortices (Walberg and Brodal, '53) in the pyramidal system. The main bundle is joined in the internal capsule by corticobulbar fibers from the frontal eye field (Beevor and Horsley, 1890; Levin, '36 Crosby, Yoss and Henderson, '52; Crosby, '53a). There are also fibers from the contralateral precentral and postcentral areas in the cat (Liu and Chambers, '64) and in monkey.

\section{Functions of motor cortex}

As Hughlings Jackson pointed out, and others have emphasized (Penfield and Rasmussen, '50), the movements elicitable from stimulation of parts of the motor cortex are those of body parts and not of body muscles. Moreover, the pattern elicitable from a small area on the motor cortex is a demonstration of the functioning of the complete neuron arcs from the specific cortical area to the terminations on the muscle fibers and not an indication of properties inherent in the cortex itself.

The motor pattern on area 4 has been altered experimentally by changing the peripheral distribution of certain nerves (Schemm and Kahn, '60; Schemm, '61; DeJonge, to be published). The area of the macaque motor cortex which gave shrugging of the shoulders was located. Then the contralateral facial nerve was sectioned and its distal end sutured into the proximal end of the spinal accessory nerve. Some months were allowed for outgrowth of fibers of the spinal accessory nerve along the branches forming originally the distal end of the facial nerve and for the establishment of nerve terminations on facial muscles. Restimulation of those portions of the motor cortex which, preoperatively, had given shrugging of the shoulders, after the nerve regeneration gave face movements. A new cortical pattern had been established.

\section{B. Supplementary motor areas}

\section{Patterns on supplementary motor areas}

Stimulation of various cortical areas, in addition to the areas along the central fissure, results in face and body movements. These regions have been described under various names such as second, additional, supplementary or extrapyramidal areas, although the terms are not quite synonymous. The wide distribution of the supplementary motor areas is indicated in part in figure 56.

The pattern of movements obtained on stimulation of the supplementary motor or extrapyramidal areas shows the same sequence as that described for the motor cortex although the dorsoventral arrangement may be reversed or otherwise altered. The movements demonstrable are usually less precise than those elicitable from the motor cortex, for they often involve all of the fingers, or the whole hand, or even most of an extremity. It is probable that the pattern on a supplementary motor area, like that on the motor cortex, indicates the functioning of the whole discharge system from the cortical level to the termination on the muscles and is not an inherent characteristic of the cortex itself.

The supplementary motor pattern on area 6 has been described by Penfield and Rasmussen ('50) for man, and by Penfield and Welch ('51) and Travis ('55) for the monkey. The patterns on the parietal areas 5 and 7 have been described for the monkey by Peele (44) and by Fleming and 


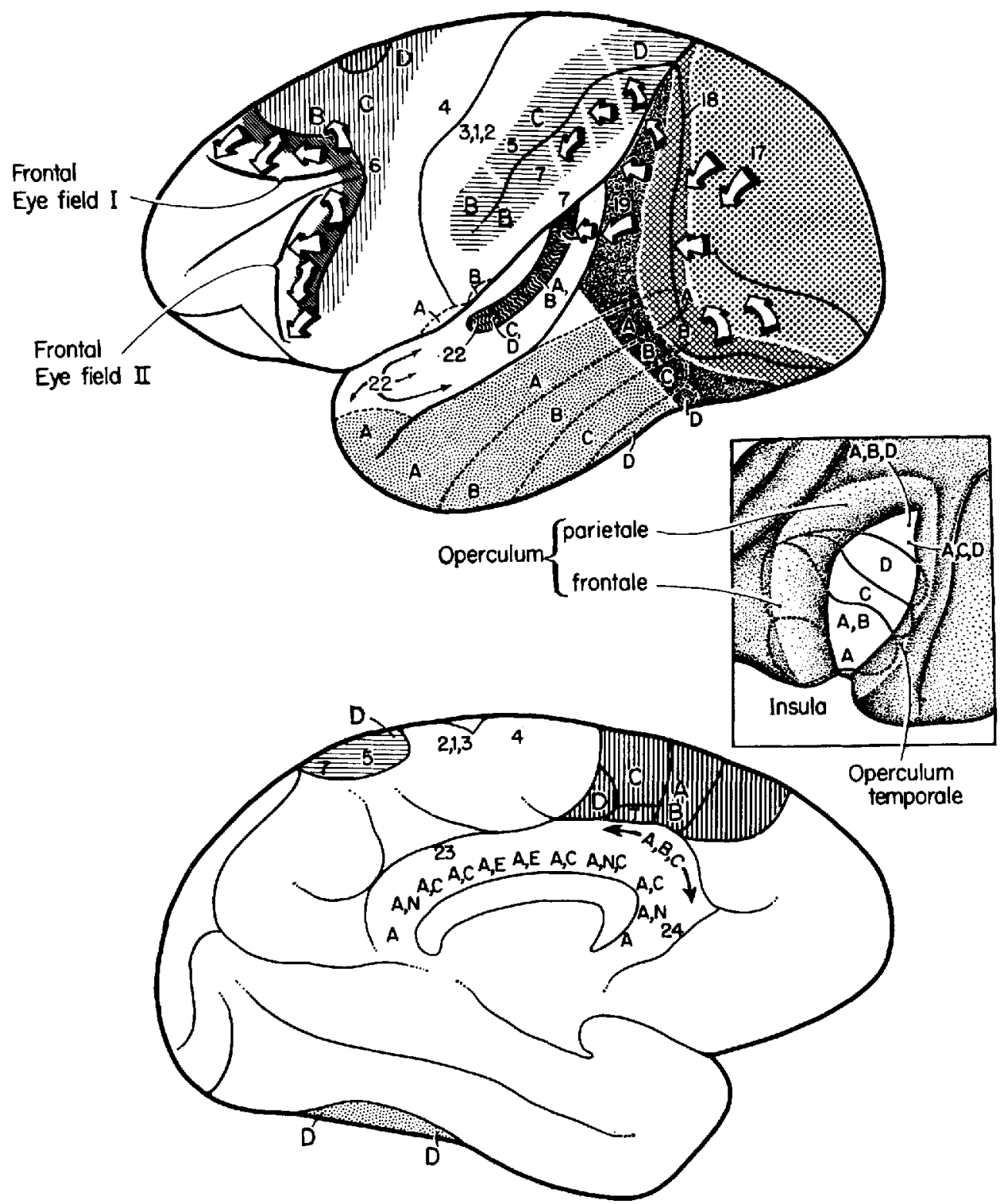

Fig. 56 Diagrams to show some of the extrapyramidal areas (indicated by number) demonstrated by stimulation of the macaque cortex. Arrows are in the fields giving eye deviation and indicate the direction of those deviations. A - movements of face ipsilateral to stimulation. B - movements of face contralateral to stimulation. C - movements of upper extremity (contralateral and sometimes bilateral). D - movements of lower extremity (contralateral and sometimes bilateral). E - movements of all extremities (contralateral and sometimes bilateral). $\mathrm{N}$ - turning of head. This fig. ure is a reproduction of figure 330 from the Correlative Anatomy of the Nervous System by Crosby, Humphrey and Lauer ('62). (Courtesy of The Macmillan Company, New York.) 
Crosby ('55). A supplementary motor area has been identified in the macaque island and the movements obtainable from it described by Frontera ('55, '56) and Showers and Lauer ('61). Respiratory changes and laryngeal movements were obtained from the rostral part of the island by Sugar, Chusid and French ('48) and Furstenberg ('58).

In both the monkey and man, adverse turning of the body and head have been obtained on stimulation of area 22 (Foerster, '31; Lemmen, '51). One of the most outstanding patterns of movements of face and body is that obtainable from the middle and the inferior temporal gyri, together with the rostral tip of the temporal lobe, in monkeys (Schneider and Crosby, '54; DeJonge, to be published). Head and neck movements appropriate to the eye deviations elicitable from near lying or overlapping areas have been demonstrated in the macaque occipital cortex by Rieck ('59). Homolateral face movements have been obtained in primates, including man (Baldwin, Frost and Wood, '54, '56), from stimulation of the amygdala and a motor pattern can be demonstrated also on stimulation of the macaque hippocampus (Votaw, '57, '59). A double pattern of motor responses is obtainable from the cingulate gyrus of the macaque (Showers and Crosby, '58; Showers, '59). Opinions have differed as to whether the responses obtainable from the posterior part of the paracentral and from the postcentral gyri should be regarded as an extension of the primary motor field in man (Penfield and Rasmussen, "50) or as constituting a supplementary motor area (Foerster, '31). Penfield and Rasmussen ('50) have described a second motor area in man at the base of the central fissure. Second motor areas in various mammals, including primates, have been described by Woolsey ('52, '58) alone and with his associates ('52).

\section{Multisynaptic descending tracts}

Although there are many reports in the literature concerning the multisynaptic pathways from various cortical areas to motor nuclei of the brain stem and the spinal cord, the knowledge of these fiber paths is far from complete. In many of the animals of the present series only cortical areas were involved in the lesions. Consequently only paths of cortical origin were degenerated. In those monkeys in which the basal ganglia were included in the lesion either these areas were only slightly invaded or, along with their major destruction, there was ablation of large cortical areas. Neither type of involvement permitted preparation of microscopic material favorable for following projection paths from globus pallidus and putamen to their terminations. None of the operations in the present series was concerned with placing midbrain lesions, so the tegmentospinal portions of the extrapyramidal systems were not degenerated. Some material has been accumulated on corticostriate, corticonigral, corticorubral, corticotegmental, and, of course, corticospinal paths. Various other components of the multisynaptic chains making up the extrapyramidal systems have been reported in other publications by the present observers (Woodburne, Crosby, and McCotter, '46; Crosby, Yoss, and Henderson, '52; Crosby, '56; Crosby, Humphrey, and Showers, '59; DeJonge, to be published).

\section{Corticostriate and corticotegmental tracts}

Most of the supplementary motor areas rostral to area 19 send corticostriate fibers to the lenticular nucleus, as well as corticotegmental fibers to the midbrain. Frequently at least, such corticostriate fibers have a larger uncrossed and a smaller crossed component, with the decussation occurring through the corpus callosum (figs. 57, 58).

Many of the corticostriate fibers from cortical areas dorsal to the plane of the lateral fissure either enter the dorsal tip of the lenticular nucleus or reach the nucleus by way of the internal or the external capsule. Such corticostriate fibers (fig. 59) have been demonstrated by degeneration techniques (Glees, '44; Berke, '60; and others) and by neuronographic methods (Dusser de Barenne, Garol and McCulloch, '42). Corticotegmental fibers from these more dorsal and more rostral portions of the cerebral cortex accompany the corticostriatal and corticospinal systems. Among those which enter the internal capsule are 


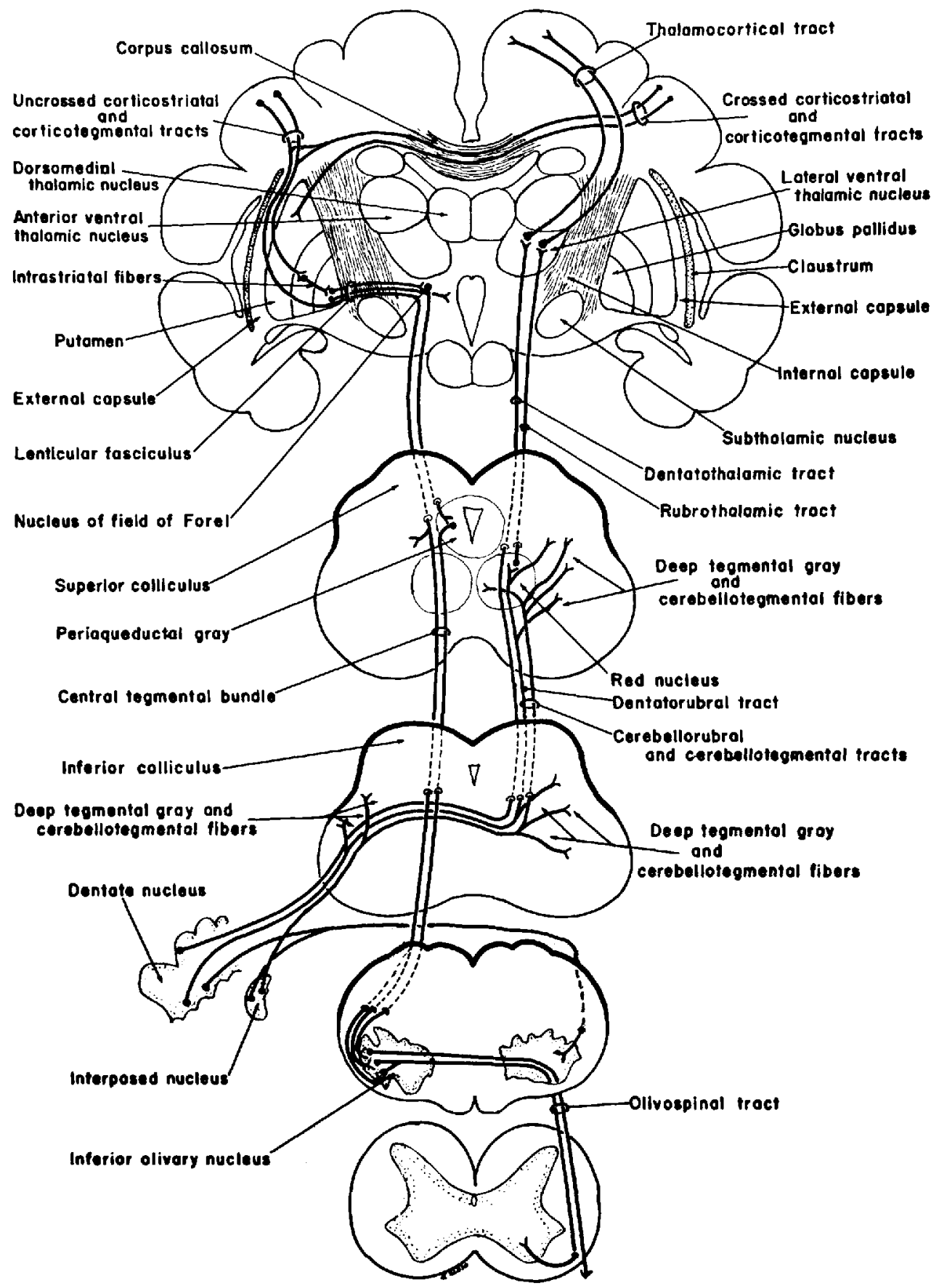

Fig. 57 On the left, the multisynaptic pathways, including corticostriate and corticotegmental fascicles, to the tegmentum of the midbrain and the inferior olivary nucleus. Diagram illustrates particularly the role played by the central tegmental bundle in this discharge system. On the right is illustrated the cerebellorubrothalamic pathway to the lateral ventral nucleus of the thalamus and the relay forward to the cerebral cortex. 
the well-known corticorubral (fig. 60) and the corticonigral (fig. 60) paths. They are illustrated in figure 60 from Marchi preparations of a brain which had a lesion involving areas 4 and 6 . A dis-

tribution of corticotegmental fibers to the deep tegmental gray is also evident in this photomicrograph.

Along with the corticostriate fascicles from the dorsal and more rostral parts of

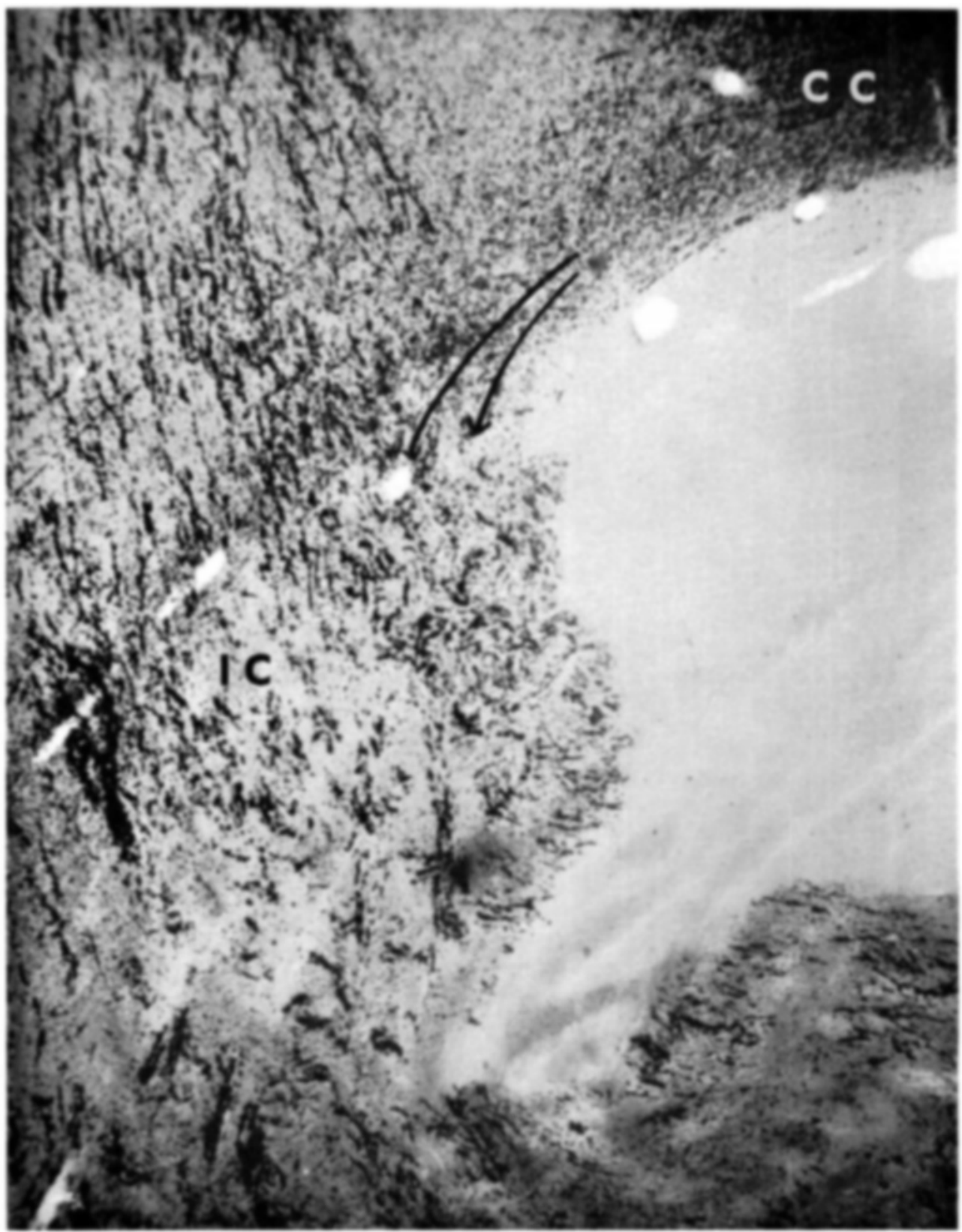

Fig. io rnotomicrograph showing uncrossed and crossed corticostriate and corticotegmental fibers. The crossed fascicles are illustrated (arrows) in their course through the corpus callosum (CC) into the internal capsule (IC), $35 \times$. 


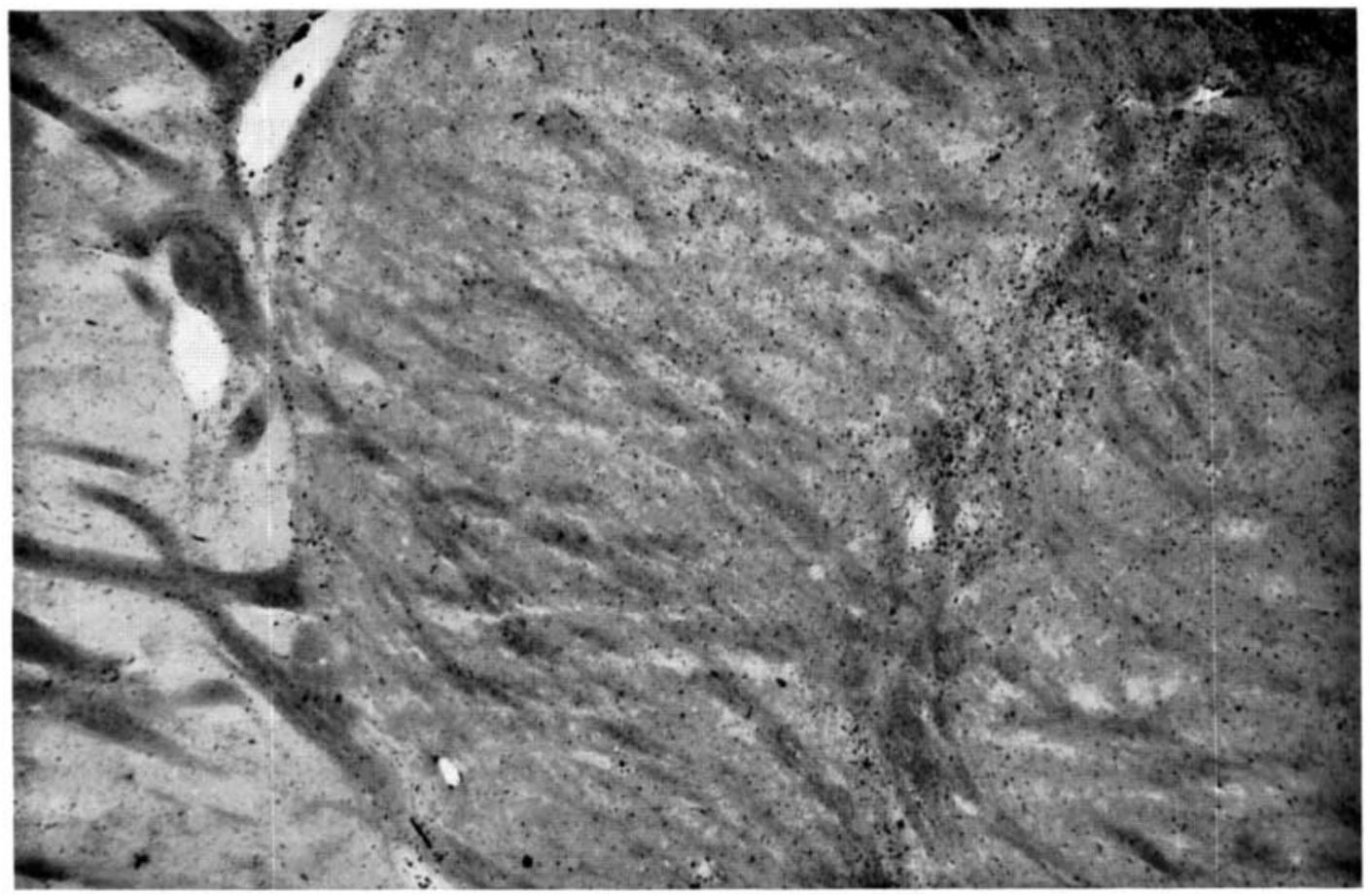

Fig. 59 Corticostriate fibers in their course from putamen into the two parts of the globus pallidus. Marchi preparation following lesion in areas 6,5 and $7,46 \times$.

the cortex by way of the external capsule are bilaterally arising corticotegmental fibers which cross through or turn under the lenticular nucleus (figs. 57,62) to join efferent systems of the nucleus (Crosby, Humphrey and Showers, '59; Berke, '60). Some of the corticotegmental fibers reach the zone incerta. Then (perhaps partly after synapse and certainly partly without such synapse) they continue back into the midbrain tegmentum. Some are found in Walberg's ('55) cortical component of the central tegmental bundle. The extent to which the corticotegmental fibers synapse in the nucleus of the field of Forel is not known. Some of them descend directly to the midbrain with the lenticular fasciculus and with fascicles from the nucleus of the field of Forel which form functionally a caudal continuation of the ansa lenticularis.

From the base of the frontal cortex, from the temporal fields rostral to area 19 , and from the lower part of the insula, corticostriate fibers enter the lenticular nucleus from its ventral side. The cortico- tegmental fascicles likewise join the ansa fibers ventral to the lenticular nucleus or cut through the lower part of this nucleus to join the lenticular fasciculus. They are known to distribute to the deep tegmental gray of the midbrain (Berke, '60).

From occipital and preoccipital cortices and, apparently, from the immediately adjoining parietal and temporal cortices, corticotegmental and internal corticotectal fibers also enter the midbrain. The corticotegmental fascicles have a postlenticular and then a sublenticular course in the monkey (Crosby, Humphrey and Showers, '59; Berke, '60). They cross through the field dorsal to the lateral geniculate nucleus to spread out in the midbrain tegmentum (fig. 61, $a$ and $b$ ). The internal corticotectal fibers pass across the pulvinar to reach the superior colliculus. The details of their distribution have been considered elsewhere and need not be repeated here (Crosby and Henderson, '48).

It has been pointed out that corticotegmental bundles reach the midbrain from 
various cortical areas. Some of these bundles, at least, spread out in the lateral part of the midbrain in accordance with a localization pattern. Fibers originating in the face areas of various supplementary cor- tical regions tend to collect in the dorsal part of the more lateral tegmental field, ventral to the superior colliculus. Those related to homolateral face movements are dorsal to those concerned with contralat-

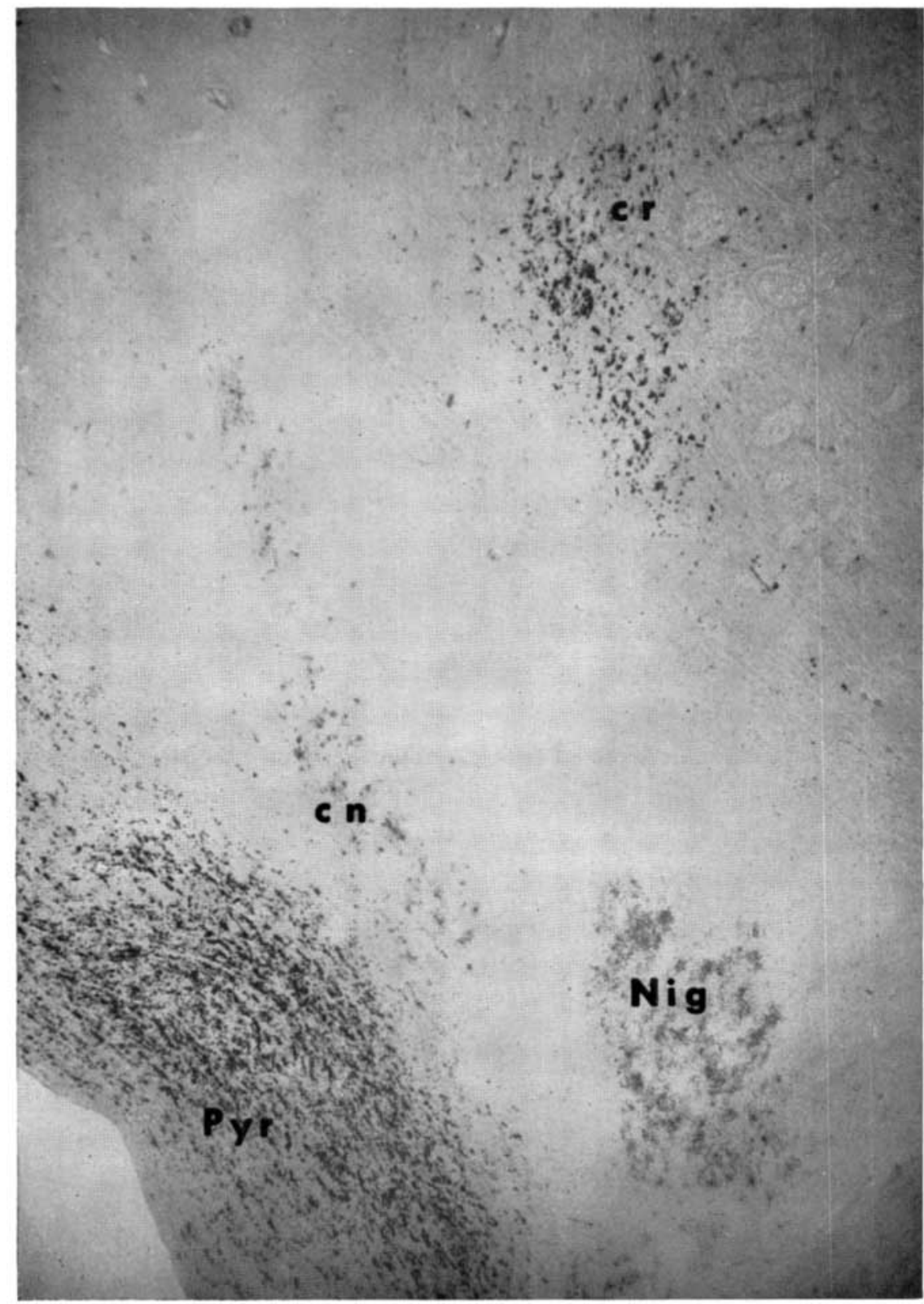

Fig. 60 Photomicrograph of the midbrain at the level of the red nucleus and cerebral peduncle showing Marchi degeneration in pyramidal (Pyr), corticorubral (cr), and corticonigral (cn) paths consequent on a destruction of the extremity regions of areas 4 and 6 on the left, $33 x$, substantia nigra (Nig). 

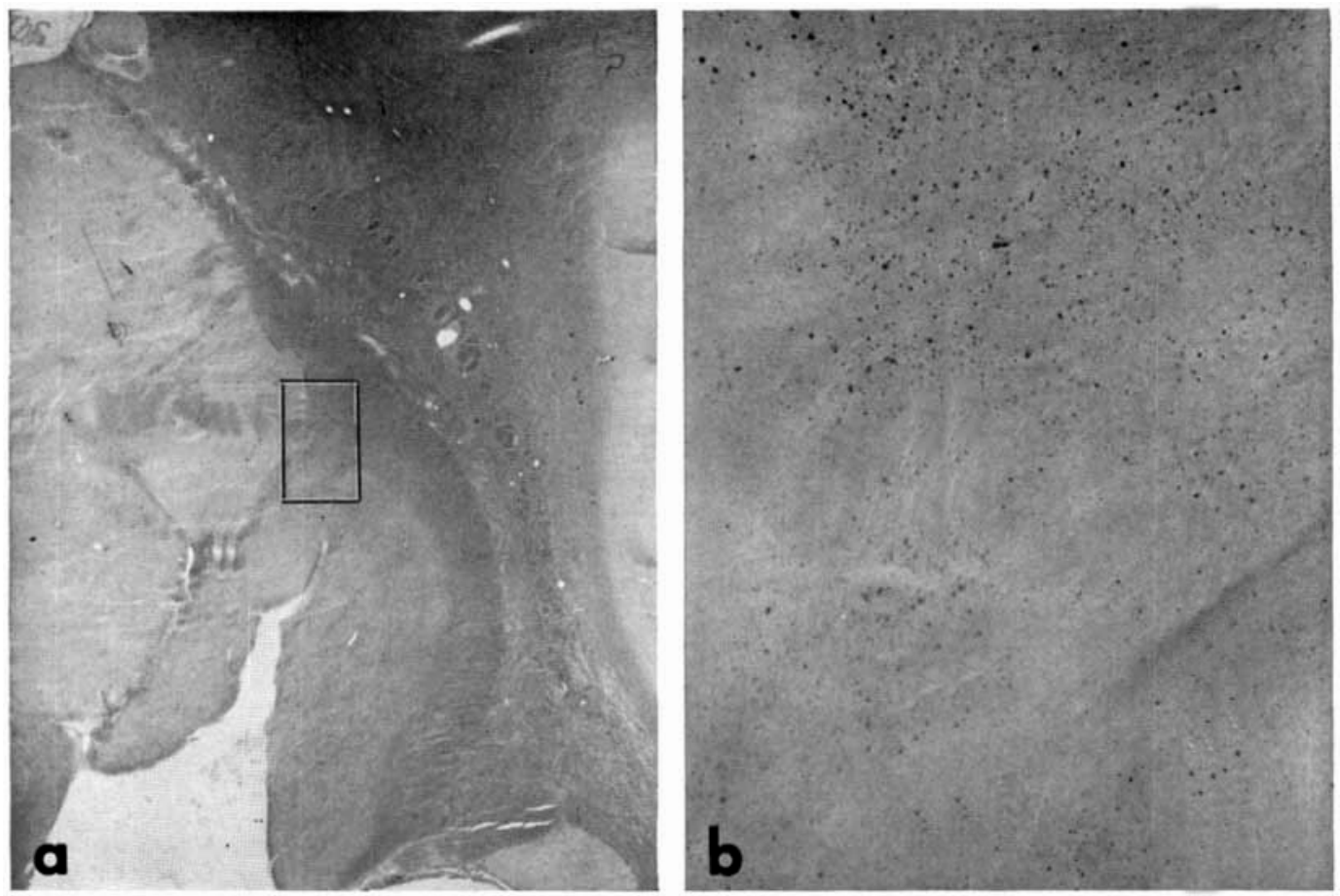

Fig. 61 (a) Section through the lateral geniculate nucleus to show the location of the enlarged area at the right, $8.8 \times$. (b) Granules of Marchi degeneration in the course of postlenticular paths traced from the posterior parietal area to the midbrain tegmentum, $76 \times$.

eral face responses. Ventral to the tegmental area from which contralateral face movements can be elicited on stimulation, and lateral to the red nucleus, fascicles collect from portions of several supplementary motor areas which give upper extremity movements on stimulation. Most ventral and slightly medialward lic the bundles which carry impulses set up in those portions of supplementary motor areas from which lower extremity movements can be elicited.

By use of the stereotaxic apparatus the parts of the corticotegmental systems can be stimulated as they course through the midbrain and a dorsoventral pattern of homolateral face, contralateral face, upper extremity, and lower extremity movements, similar to those seen on comparable testing of the supplementary motor areas, can be demonstrated (Carey and Crosby, to be published). This midbrain pattern is very similar to that demonstrated by Hess and his colleagues (Hess, Bürgi, and Bucher, "46) in the cat.

Descriptions of corticostriate fibers are available in the literature from many supplementary motor areas such as area 6 (Dusser de Barenne, Garol and McCulloch, '42; Berke, '60), island cortex (Rae, '54), various temporal areas (Segundo, Naquet and Arana, '55; Crosby, '56; Berke, '60; Schneider and Crosby, '60; DeJonge, to be published), parietal cortex (Le Gros Clark, and Northfield, '37), cingulate cortex (Showers, '59), orbital cortex (Berke, '60), and somesthetic sensory cortex (Dusser de Barenne, Garol and McCulloch, '42; Peele, "44).

Corticorubral fibers (fig. 60) have been described from areas 6 and $4 \mathrm{~s}$ by Levin ('49). A corticonigral tract (fig. 65) has been identified by v. Monakow (1895), Mellus (1899), Minkowski ('23, '24), Hirasawa and Kariya ('36), Levin ('36, '49), Verhaart and Kennard ('40), and many others. Corticotegmental (reticular) fibers have received much documentation from many sources and by various techniques (Hirasawa, Okano, and Kamio, '38; Crosby and Henderson, '48; Levin, '49; Lemmen, '51; French, Verzeano, and Magoun, '53; Showers, '59; Crosby, Humphrey, and Showers, '59; Berke, '60; and others). 


\section{Basal ganglion components}

The efferent pathways from the basal ganglia have been reported by many observers and are undoubtedly very familiar to most readers (fig. 62). Since they represent one of the most important portions of the multisynaptic discharge paths from various supplementary motor areas to primary motor centers by way of the basal ganglia, they are briefly reviewed and documented in the following small type. These pertinent efferent connections include the lenticular fasciculus, the subthalamic fasciculus, and the ansa lenticularis.

Lenticular fasciculus, The lenticular fasciculus of Forel (1877) arises primarily from the inner segment of the globus pallidus but has some accompanying fibers of a cortical origin (Berke, '60; Crosby, Humphrey and Showers, '59; Showers, '59). Such fibers (fig. 62) arise bilaterally from various supplementary motor areas, having undergone a partial crossing in the corpus callosum This cortical component passes Iargely by way of the external capsule. It then crosses the lenticular nucleus to join the other fibers of the lenticular fasciculus. From the globus pallidus, the fascicles of the lenticular fasciculus interdigitate with the bundles of the posterior limb of the internal capsule and pass medialward in relationship to the zona incerta and above the subthalamic nucleus to reach the nucleus of the field of Forel. There is some synapse in the region of the zona incerta and some of the cortical fibers pass caudalward (fig. 57) to join the central tegmental bundle (Walberg, '55). In the region of the nucleus of the field of Forel, there is very considerable relay. Some observers (for example Ranson and Ranson, '39; Papez, '42) considered that all of the fibers terminate in this nucleus. Others (Laursen, '55; Woodburne, Crosby and McCotter, '46) thought that fascicles, perhaps especially those of cortical origin (Berke, '60), joined by many fibers from the nucleus of the field of Forel, turn caudalward to end in the nucleus of Darkschewitsch (Woodburne, Crosby and Mccotter, '46; Laursen, '55), in the interstitial nucleus of the medial longitudinal fasciculus (Foix and Nicolesco, '25; Jakob, '23; Morgan, '27; Woodburne, Crosby and McCotter, '46), in the red nucleus (Riley, "43; Woodburne, Crosby and McCotter, '46; Laursen, '55; Neumann, '59), and in the deep tegmental gray dorsolateral to it (Woodburne, Crosby and McCotter, '46).

Subthalamic fasciculus. Whittier and Mettler ('49a) listed some twenty-two observers who had described connections between the basal ganglia and the subthalamic nucleus and this number could be considerably increased. Evidence that the fascicles are both afferent and efferent to the subthalamic nucleus (fig. 65) and an interesting account of experimental lesions in this nucleus in monkeys are also given in the publications by Whittier and Mettler ('49a,b). From the subthalamic nucleus relay is made to the tegmentum of the midbrain by the subthalamo- tegmental tract (Papez, '46; Woodburne, Crosby and McCotter, '46).

Ansa lenticularis. The ansa lenticularis (fig. 62) arises chiefly from the putamen and the globus pallidus. Accompanying it are cortical components from various supplementary motor areas. These are partly of ipsilateral and partly of contralateral origin. The ansa lenticularis enters the diencephalon ventral to the internal capsule and swings dorsomedialward, along the medial side of the subthalamic nucleus, to the region of the nucleus of the field of Forel. There is a great difference of opinion among the many observers (Wilson, '14; Morgan, '27; Papez, '42; Ranson and Ranson, '39, '42; Ranson, Ranson and Ranson, '41; Laursen, '55; Woodburne, Crosby and McCotter, "46; Neumann, '59; and many others) who have described this bundle as to whether its fascicles have total or subtotal synapse in the nucleus of the field of Forel. Some investigators (for example, Neumann, ' 59 ) have believed that all the fascicles of the ansa end in this nucleus. Others have felt that some fibers (Woodburne, Crosby and McCotter, '46), quite possibly the cortical component (Berke, ' 60 ), pass through the nucleus without synapse. The present study has nothing to contribute along this line. In any case, fascicles extend from the region of the nucleus of the field of Forel into the tegmentum of the midbrain to end in the reticular gray ventral to, and also caudal to, the red nucleus.

\section{Tegmentospinal and reticulospinal components}

Various tegmentospinal and reticulospinal paths (figs. 62, 65) are common discharge systems to motor centers, for impulses relayed to midbrain and bulbar reticular gray from cerebral hemispheres and cerebellum. These paths are discussed very briefly under the consideration of certain cerebellar efferent systems (p. 75).

\section{Functions of supplementary motor areas}

In the normal monkey, or in man, the supplementary motor areas are concerned in the production of cortical automatic associated movements. There is a great array of such movements, which accompany and give character and color to the more precise and the more highly specialized (but often less distinctive) so-called voluntary responses. The superb fingering of the skilled violinist is an example of functioning of the primary motor cortex but soft tapping of his foot or the swing of his body in time to the music, and, perhaps, the expression on his face represent cortical automatic associated movements. Many of the supplementary motor areas 


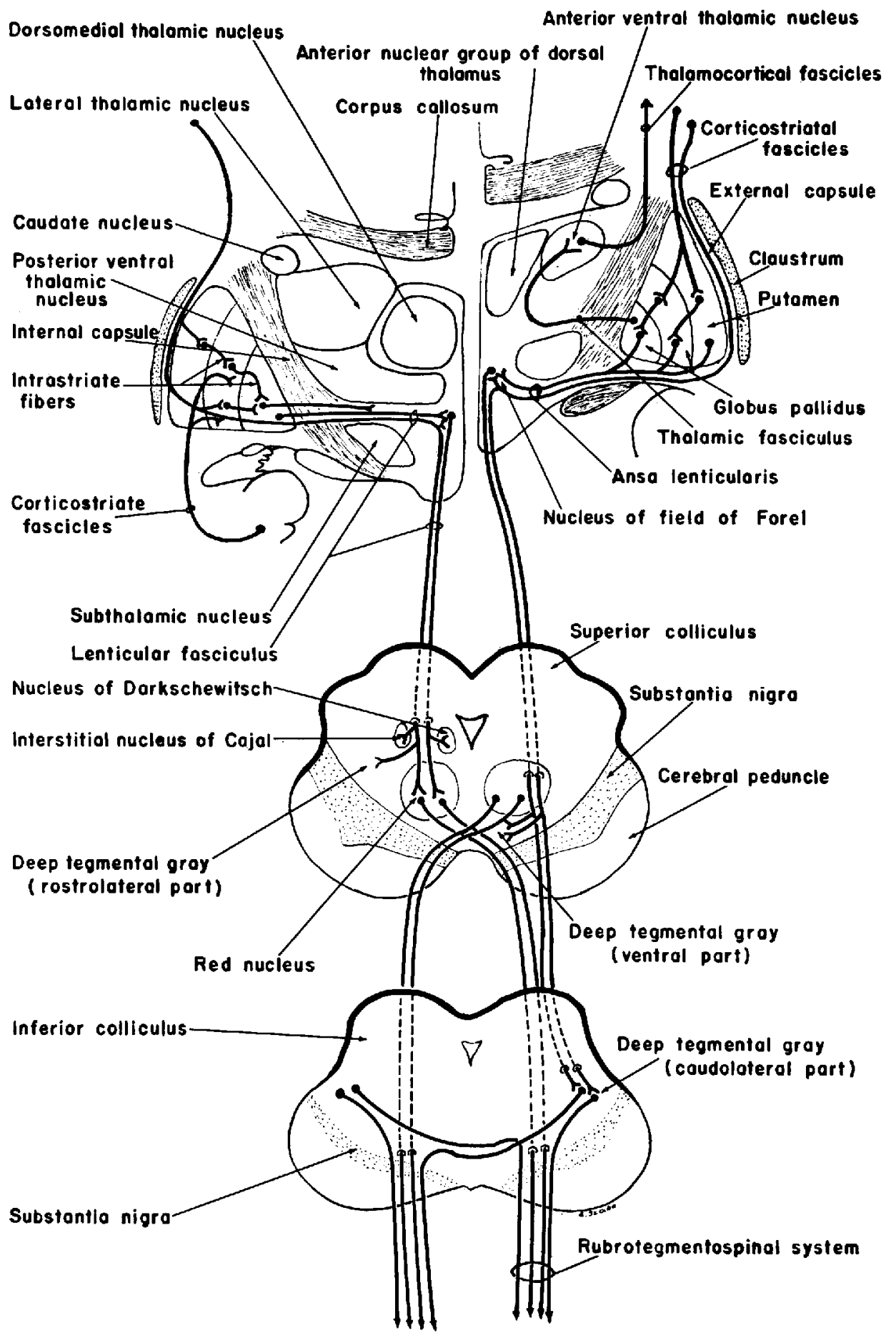

Fig. 62 Diagram illustrating major components of the lenticular fasciculus and of the ansa lenticularis. The fibers related to the ansa by way of the external capsule are illustrated. Corticotegmental fibers are included in the bundle labeled corticostriatal fascicles. 
are located in association cortices and the cortically initiated movements are indicative of the efferent arcs from these association areas.

The interrelations built up in the association areas of the monkey or of man differ not only from one individual to another but may vary during the lifetime of the same individual. Consequently, the emotional reactions and the personality are revealed by the individualistic motor and autonomic responses set off by stimulation of arcs relaying impulses from the supplementary motor areas through the tegmentum of the midbrain or through the hypothalamus to lower motor and autonomic centers for the regulation of their discharges.

To avoid misunderstanding, perhaps the well known fact should be mentioned (DeJong, '58; Grinker, Bucy and Sahs, '60) that automatic associated movements of more primitive phylogenetic significance, such as swinging the arms in walking, are thought to be due to basal ganglion functioning and not to require cortical participation.

Lesions in the globus pallidus (such as may be present in Parkinsonism), however, produce an individual with a masked or expressionless face, since this region is an area of convergence of many efferent paths from supplementary motor areas.

\section{Resumé of motor deficits}

Unilateral lesions in the extremity regions of area 4 of the monkey produced a permanent contralateral loss and bilateral lesions a permanent bilateral loss of fine movements of the extremities, demonstrable at their distal ends and particularly in the fingers and the toes. Bilateral lesions of the so-called face region (which actually includes those portions of the motor cortex activating the primary motor nuclei of cranial nerves) resulted in a bilateral loss of voluntary movements of the face, the tongue, and the pharyngeal muscles (and presumably of the striated muscles of the larynx, the uvula, and the esophagus, which were not tested), so that there was difficulty in eating and swallowing. In man, there is a contralateral paresis of the lower part of the face, the uvula, and the tongue following destruction of one motor "face" area. Destruction of area 4 does not produce abnormal eye deviations or interfere with voluntary eye movements. Some observers have regarded the eye movements elicitable from area 4 as of the nature of cortical automatic associated movements (Sherrington, 1893; Crosby, Yoss, and Henderson, '52; Crosby, '53a).

Injuries to frontal eye fields resulted in a more or less transient inability to turn the eyes away from the side of the lesion or perhaps almost equally transient deviation of the eyes toward the side of the lesion due to the imbalance between the discharges of the uninjured and the injured eye fields on the two sides of the brain. A patient with a small unilateral lesion which involves only the portions of the frontal cortex related to obliquely upward deviations of the eyes toward the opposite side, sometimes shows forced deviations obliquely upward toward the side of the lesion. Bilateral destruction of the frontal eye fields is indicated in the monkey, or in man, by an inability to turn the eyes voluntarily in any direction, although following eye movements and eye reflexes are intact.

When lesions involve specific supplementary motor or extrapyramidal areas, motor deficits (or signs suggesting such deficits) have been obtained only from certain areas. Thus unilateral destruction of both preoccipital and occipital eye fields (but not of one field alone) in monkeys resulted in a loss of following eye movements away from the side of the lesion (see also, Crosby, Yoss, and Henderson, '52). Bilateral destruction of these areas (or bilateral destruction of the visual cortex since some degree of vision is necessary) produced a loss of optokinetic responses in all directions. Following eye movements were not present in the animals which had had bicortectomies. The inhibitory effects of the discharges of the frontal eye fields over the following eye movements (elicitable on stimulation of the preoccipital and occipital eye fields by impulses from the visual cortex) were seen in the to-and-fro movements of a monkey's eyes after extensive bilateral frontal lobe ablations and the ease with which optokinetic responses were obtained by use of an optokinetic drum (Henderson and Crosby, '52). Sometimes 
blinking could be demonstrated in the monkeys with cortical blindness. There is some question in the observers' minds as to whether such blinking was due to visual stimuli or was the result of tactile stimulation produced by the air currents set in motion by the sudden movements of the examiner's hand or to temperature stimuli from the heat of the flashlight used in testing. Such blinking may be interpreted as indicating that this reflex is mediated by pathways that synapse in the lateral geniculate nucleus and the superior colliculus in their course to the oculomotor nuclei. It would not require cortical participation and so not be affected by bilateral destruction of the visual cortex. Light reflexes, which require only intact circuits through midbrain centers, were present in monkeys with total bicortectomies. Unilateral lesions involving the visual cortex produced a contralateral homonymous hemianopia and bilateral destruction of this type a cortical blindness.

Unilateral destruction of part or all of area 6 in the monkey often produced no motor defects which the observers recognized. Often no grasp reflex was elicited after such an ablation (see Travis, '55). Destruction of area 6 is believed to produce a grasp reflex in the contralateral hand and foot in man. Bilateral destruction of area 6 does produce a transient grasp reflex in monkeys but, if both cortical areas are not removed in the same procedure, the grasp reflex may only appear on the side contralateral to the second operation. In a few monkeys in which not only the hand part of area 6 but some of the cortex adjoining it rostroventrally was also involved, the movements of the contralateral hand were clumsy (although there was no actual paresis) suggesting a possible frontal lobe apraxia.

A lack of positional sense, particularly noticeable on the extremities, and most especially on the hand and the foot on the side opposite the lesion, characterized injuries to somesthetic cortex. Except in the occasional monkey, in which curiosity motivated the animal to examine an object closely - and to shift it to the unaffected hand for such an examination - evidence for a loss of discriminatory tactile sensibility from removal of the contralateral somesthetic cortex was not obtained. The evidence for loss of the more discriminatory tactile sensibility is then entirely equivocal in the monkeys reported in this paper. There is no doubt that the child with a subtotal hemispherectomy had a loss of two-point tactile and other highly discriminatory types of tactile sensibility and of positional sense on the hand and the foot on the side contralateral to the operation.

Parietal lobe apraxias were noted in some of the animals. In two monkeys, following destruction of areas 5 and 7 , there was a suggestion of a loss of body scheme. This was not related to handedness, since most of the monkeys were ambidextrous before operation, although they all favored the hand on the side of the lesion after a unilateral ablation of the hand area of the motor cortex.

Changes in personality were noticeable in monkeys with large cortical lesions, particularly perhaps in those with cingulate or frontal lobe destruction. The cingulate monkeys tended to be hyperkinetic and overly curious. The hair (Ward, '48) was erected over their backs and, particularly, over their shoulders. The monkeys with large frontal lobe lesions showed typical repetitive, stereotyped movements. After a one-sided ablation, they circled toward the side of the involvement. Apparently these stereotyped movements remain a permanent part of the animal's behavior since they were present long after the operative procedure which produced them. They were very evident in one monkey three years after a hemicortectomy, appearing whenever he was excited.

A monkey with a hemicortectomy or a hemispherectomy is a quite capable, fairly intelligent animal. The child with a subtotal hemispherectomy was able to carry out her studies and graduate from high school. As the lesions are further increased, by bilateral involvement, the general status of the animal or the child is lowered. Cerebral palsy children with widespread, bilateral cortical lesions have, almost invariably, subnormal intelligence. Following subtotal or total bicortectomies, the monkey, although apparently not in coma, was indifferent to his surroundings, unless he was hurt, and was completely 
unambitious and unintelligent. He did respond to painful stimuli with a cry and a series of jerks and might jerk on being touched. Even when his opisthotonos was lessened by cerebellar lesions, he made no attempt to help himself in any way. When he was touched, his limbs moved in a coordinated fashion (because of intact arcs from the thalamus through the basal ganglia and back to lower motor centers), but he made no effort to turn, or to move about, or to try to feed himself. There is every contraindication to performing a hemispherectomy on a child in which there is evidence of destruction in the cerebral cortex on the side opposite the intended removal.

\section{Substitution in cortical motor activity}

It is clearly evident that permanent loss of fine movements follows unilateral or bilateral involvement of the motor cortex in correspondence with the amount and the location of the destruction and that voluntary eye deviations depend on frontal eye fields. However, it appears that reasonably effective, intelligently controlled movements are possible in monkeys and, to lesser extent, in man after bilateral removal of area 4 or after a hemicortectomy. It is suggested that such movements become possible through the partial substitution of the discharges of supplementary motor areas (over their multisynaptic efferent paths) for those of the primary motor cortices (mediated by the more direct pyramidal systems). The monkeys learned to walk again, to feed themselves and to carry on various activities after bilateral destruction of the regions along the central fissure. They were utilizing the grosser movements still elicitable from the supplementary motor areas. The cerebral palsy child with bilateral spastic paresis can perform some useful movements when his tonus is lessened.

However, the substitution is not always so direct as replacing finer by relatively coarse movements. It often takes the form of replacing one kind of movements by another. An example is the substitution of following eye movements for voluntary eye movements. As has often been noted removal of the frontal eye fields bilaterally in either monkey or man leads to a perma- nent inability to turn the eyes voluntarily in any direction of gaze. This disability is particularly annoying in reading. Patients who can not turn the eyes voluntarily in the horizontal plane are taught to place a finger at the beginning of a line and move it steadily across the page under the line. The eyes will follow the moving finger along the line, back to the beginning of the next line, and so down the page. Another partial substitution is that of automatic and/or emotional smiling for voluntary smiling. Voluntary smiling is lost on the side opposite a destruction of the face area of the primary motor field. Emotional smiling and automatic smiling are not lost for they are functions of certain limbic and other supplementary motor areas and their discharge paths. The patient, then, who cannot smile on command does not have an expressionless face for he can still smile at an amusing story (Monrad-Krohn, '24).

\section{Rôle of association cortices in motor activity}

The motivating impulses for carrying on highly voluntary movements of the body and the head are not built up initially in area 4 or in the primary frontal eye fields but in the association cortices. The motivation for motor performances of all types remains after destruction of the primary motor fields of the cerebral cortex. Such association areas not only activate the motor cortices but they also fire the supplementary motor fields to provide for the automatic associated movements which accompany the more specific motor acts. These connections with the supplementary motor fields will remain after the primary motor areas or their discharge paths, the corticobulbar and corticospinal components of the pyramidal systems, are destroyed.

Destruction of a single supplementary motor area, unilaterally or bilaterally, may or may not give rise to any untoward motor signs. For example, unilateral destruction of area 6 produces a grasp reflex in the contralateral extremities in man and at least sometimes in monkeys. In bilateral ablations of area 6 grasp reflexes are present in both monkeys and man. Unilateral destruction of the eye fields in areas 18 
and 19 [but not in only one of these areas (Crosby, Yoss, and Henderson, '52)] results in a loss of the following eye movements away from the side of the lesion.

Many supplementary motor fields constitute the efferent portions of association fields. When an association field concerned with building up visual, or auditory or somesthetic association patterns is involved in a lesion, the afferent effects are so marked that no attention is paid to the loss of related automatic associated movements. Afferent impulses relayed to the cortex are often rendered meaningless by lesions involving the association cortex bordering the receptive cortical areas. An illustration is afforded by the effects of the rare bilateral lesions, or unilateral destruction in the dominant hemisphere, of the portions of area 22 bordering areas 41 and 42 . Area 22 , in the region mentioned, serves as an auditory association cortex but also functions as a supplementary motor area (Foerster, '31; Lemmen, '51). When this auditory association area (supplementary motor field) is destroyed, the evident defect is an auditory aphasia.

When all the association areas (with their efferent discharges) are destroyed in one hemisphere, as in the hemicortectomies and the hemispherectomies reported for monkey and man, or even when there is considerable bilateral cortical destruction in these primates, they still make some valuable adjustments to their surroundings. However, the effects of major bilateral or total bilateral cortectomies in the monkey are calamitous. That irrevocable deficits seen under such circumstances are due to the loss of the association areas is documented in man by the behavior and the capabilities - or lack of them - seen in Pick's disease. In this disease, those cortical areas concerned with the reception of impulses set up by auditory, visual, and like stimuli and area 4 are usually spared and the association areas are degenerated. Such patients may be able to move individual fingers and toes and have, in general, fine movements but they show no type of intelligent behavior.

It may be concluded that a monkey or a man with a bilateral removal of the primary motor areas of the cerebral hemi- spheres may still have a useful and a productive life. Any primate with complete destruction of supplementary or extrapyramidal areas (since many of them fall in association areas) has reached a point from which there is no return to any type of useful existence.

\section{Regulation of tonus at reflex levels}

There are many ways in which one may discuss tonus and many levels of the nervous system which are involved in provid ing adequate tonus for the great range of primate motor performances. Basic for all tonus are intact motor neurons. There are two types of efferent fibers (alpha and gamma fibers) in the motor roots which terminate on muscle fibers of striated voluntary type. The alpha fibers arising from the large ventral horn (alpha) neurons of the cord (and the corresponding neurons of cranial nerves, undoubtedly) supply the striated muscle fibers. Stimulation of the alpha fibers produces contraction of the muscle. The smaller gamma fibers arise from smaller neurons. They pass out in the motor roots to distribute to striated muscle but they terminate upon the small intrafusal muscle fibers of the muscle spindles. Contraction of the intrafusal fibers leads to a stretching of the "noncontractile" nuclear bag region where the muscle fibers are non-striated (and noncontractile, Ruch et al., '61) and a stimulation of the annulospiral endings situated at this midportion results. These annulospiral endings are the nerve terminations on the large medullated processes (A fibers) of the large proprioceptive neurons, the cell bodies of which are located in the appropriate spinal ganglia and the axons of which end directly on the alpha neurons. Evidence that this is a monosynaptic arc has been provided by Lloyd (' 43 ). Stimulation of these proprioceptive neurons results in facilitation of the homonymous alpha neurons with a contraction of the extrafusal muscle fibers they supply and an inhibition of the impulses over the alpha neurons supplying the muscle opposing such action (i.e. the so-called antagonist). The contraction of the extrafusal muscle fibers lessens the tension on the muscle spindles so that the intrafusal 
muscle fibers relax and the spindle receptors cease firing (Glees, '61; Ruch et al., '61). The intrafusal and the extrafusal muscle fibers may not contract at the same time. However, concomitant activation of the intrafusal muscle may occur during contraction of the extrafusal muscle and then a firing of the spindle may take place during extrafusal muscle contraction (Ruch et al., '61).

Various other stimuli - particularly cutaneous stimuli - alter the fusimotor discharge. Evidence for the relay from cutaneous fibers to gamma as well as to alpha neurons has been provided by various observers (Granit, Job and Kaada, '52; Granit, '55; Hunt and Paintal, '58). For some regions of the body for which the effects of such stimuli have been tested (as for the foot, Ruch et al., '61), the extensor muscles on the side contralateral to the stimulus have an increased fusimotor discharge and the flexors a decreased discharge but on the side of the stimulation the relations are reversed.

Hunt and Paintal obtained facilitation of the gamma neurons supplying the anterior tibial muscle, and inhibition of those supplying the ipsilateral extensor longus muscle, when they applied tactile stimuli to the cat's paw. This provided for coordinated contraction and relaxation of these msucles. However, such interrelations are not invariable for all body levels. Stimulation of the gamma fibers, then, as Leksell ('45), Hunt and Kuffler ('51a, b), and others have pointed out, produces an increased rate of discharge of the proprioceptive neurons having annulospiral endings in the muscle spindles. According to Glees ('61), there is less passive regulation of the rate of muscle spindle discharge from stretching the muscle fibers than active regulation of this rate by discharge of the gamma fibers.

The neurotendinous endings show an increased firing during extrafusal muscle contraction. They are believed to exert an inhibitory effect over the alpha neurons. According to Eccles, Fatt and Landgren ('56a, b) this is by way of a disynaptic arc with an intercalate (or interposed) neuron in the arc. Lloyd ('46) had found no evidence for such an interposed neuron. Moreover, a special group of intermediary or interposed cells, known as Renshaw cells (Renshaw, '46, and elsewhere), is believed to be activated by collaterals of motor neurons. In this activation, it is postulated that impulses from various motor neurons are relayed to a single Renshaw cell (Eccles Fatt and Landgren, '56a); in turn the Renshaw cells make "inhibitory synaptic contacts with motoneurons at the same segmental level" (Eccles, Fatt and Landgren, '56b, p. 79).

Perhaps it should be emphasized that the stretch reflex may be elicited without the gamma fibers coming into play. They represent particularly the final common neurons by which the muscle spindle and so the stretch reflex are placed under the control of higher centers, and consequently the tonus of the motor responses are regulated.

A transection of the spinal cord, which of course removes the reflex arcs caudal to the injury from the regulatory effects of higher centers, results in a bilateral spastic paralysis (a paraplegia or a quadriplegia, depending upon the level of the injury). For example, a transection of the cord above the lumbar enlargement will produce a spastic paraplegia, usually, but not invariably, a paraplegia in flexion.

\section{Postural tonus at cerebellar levels}

\section{A. Certain cerebellar relations and connections}

\section{Afferent connections}

From spinal cord and brain stem areas which receive impulses set up in muscles, tendons, and joints, and at cutaneous surfaces, from visual and auditory centers within the brain stem, and from the peripheral vestibular apparatus and the vestibular centers of the medulla and the pons, there are discharges to the cerebellum. Space does not permit a detailed account of these projections (Jansen and Brodal, '54, '58; Dow and Moruzzi, '58; Crosby, Humphrey and Lauer, '62; Brodal and Drabløs, '63), which have been recently reviewed in some detail by one of the present observers. Whatever other functions may also be assigned to them, it seems quite certain that, by discharge and redischarge through the Purkinje cells of the cerebellar cortex (Ramón y Cajal, '09-'11; 
Fox and Barnard, '57), particularly that of the posterior lobe, impulses are built up for the maintenance of normal posture and the tonus necessary for maintaining that posture. The spontaneous activity of the Purkinje cells, which has been demonstrated by use of microelectrodes for intracellular recording, probably is interrelated, as Granit and Phillips ('56) suggested, to the regulation of muscle tonus.

\section{Corticonuclear fibers}

The cerebellar cortex discharges to thalamic, brain stem, and spinal cord levels, largely after relay in the cerebellar nuclei. The corticonulear fibers represent axons of Purkinje cells.

The possible relation (Jansen and Brodal, '40, '42) of a specific region of the cerebellar cortex to specific cerebellar nuclei (or even to particular parts of such nuclei) is of great interest (fig. 63). It has frequently been stated that, in general, this relation was of a radial type, with the cerebellar cortical areas distributing to the portions of the nuclear gray lying most nearly deep to them. According to this idea, the cortex on one half of the vermis should project to the fastigial nucleus, that of the lateral part of the posterior cerebellar lobe to the lateral part of the dentate nucleus, and the intervening regions of the cortex and the nuclear gray should have comparable relations (Jansen and Brodal, '40, '42; Walberg and Jansen, '64). Experimental studies on various mammals suggest that,

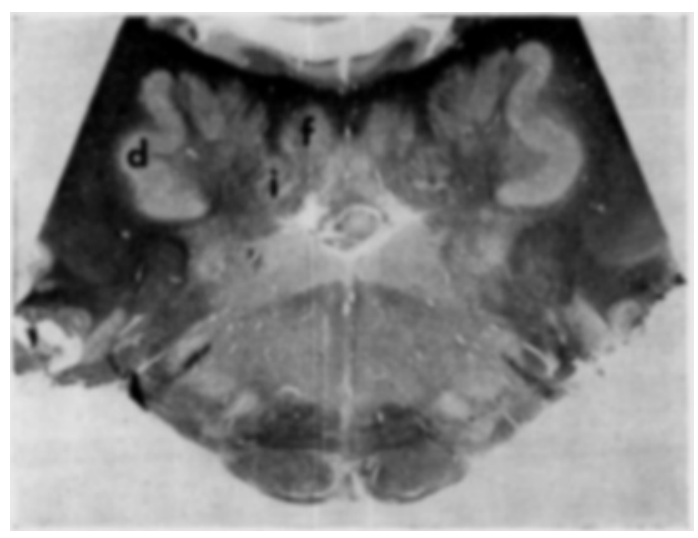

Fig. 63 Weil preparation showing the very large cerebellar nuclei in the macaque, termed here nucleus fastigii ( $f$ ), nucleus interpositus (i), and nucleus dentatus (d). although there are certainly some surfaceto-nuclear gray relations of the sort just suggested, a completely simple radial arrangement of all corticonuclear fibers from the cerebellar cortex to the cerebellar nuclei can not be documented. Thus, in the rat, Goodman, Hallett and Welch ('63) and, in the cat, Eager ('63) found the corticonuclear fibers which arose from one cortical zone distributing in a pattern to two or three cerebellar nuclei, with each group of fibers showing a particular pattern of distribution. Studies on the monkey (Vachananda, '59) indicated that, although the discharge from the vermal cortex was chiefly homolateral to each fastigial nucleus, there was evidence, from Marchi preparations, for the existence of crossed corticofastigial fibers.

\section{Efferent connections}

The efferent discharges from the cerebellar nuclei (fig. 63) are primarily over paths which constitute components of the inferior and the superior cerebellar peduncles. Obviously it is impossible to discuss all of these efferent paths, nor would it serve any real purpose. Those of particular significance are documented briefly.

Inferior cerebellar peduncle. The inferior cerebellar peduncle carries various efferent paths which arise from the fastigial nuclei. (There may also be some fascicles originating in the cerebellar cortex.) Among such components are decussating fibers to the cervical cord which form the cerebellospinal portions of the hook bundle. The inferior cerebellar peduncle also carries cerebello-reticular, cerebello-olivary, and cerebello-vestibular fibers (Walberg and Pompeiano, '60; Walberg et al., '61, '62a, b). The cerebello-reticular and cerebello-vestibular fibers are of particular interest because they are parts of arcs related to the maintenance of equilibrium, which is disturbed in posterior vermis lesions. The unequal involvement of the vermal cortex, or the fastigial nuclei, or the cerebello-vestibular fibers on the two sides of the brain underlies the nystagmus which appears in partial involvements of the posterior vermis.

Cerebello-vestibular interrelations are very important in the maintenance of equilibrium and in certain righting re- 
flexes. The monkeys in the present series that had large lesions in the posterior vermis and to some extent the patients on which some of the operations were carried out showed interference with these connections and some of the disturbances in maintenance of equilibrium and difficulties with head righting to be expected with involvement of such vestibular-cerebellar interrelations. The following contributions quoted from the great wealth of material on this subject offer an approach to the literature. These contributions are papers by Bruce (1895), Löwy ('16), Rasmussen (32), Dow ('36, '38), Larsell (37), Spiegel and Sommer ('44), Schneider, Kahn and Crosby ('51), Crosby ('53a, b), Jansen and Brodal ('54,'58), Cohen, Chambers and Sprague ('58), Dow and Moruzzi ('58), Walberg and Jansen ('61), Brodal and Drabløs ('63), Brodal ('64), and Brodal and Høivik ('64).

The literature on cerebello-reticular relations is also large. Among some of the many papers which may be cited are those by Blakeslee, Freiman and Barrera ('38), Rand ('54), Jansen and Jansen ('55), Brodal ('56), Thomas et al. ('56), Walberg ('58), and Walberg and his associates ('60, '62a, b).

Superior cerebellar peduncle. The superior peduncle (figs. 57,65) is directly involved in some of the lesions reported in this series. Its efferent components, which arise, to a small extent, from the fastigial nucleus (Carrea and Mettler, '54) but chiefly from the other cerebellar nuclei, are major discharge paths from the cerebellum. They include: (1) paths to the contralateral cerebral cortex and the basal ganglia by way of a synapse in the contralateral nucleus ventralis lateralis of the dorsal thalamus; (2) bilateraly distributing fascicles to the brain stem reticular gray, particularly to the tegmental gray of the midbrain; (3) a cerebellorubral tract, which terminates, at least to a major extent, in the magnocellular and parvocellular portions of the red nucleus on the side opposite to the origin of the tract; and (4) the flocculo-occulomotor path, which ends bilaterally in the occulomotor nucleus. A destructive lesion in the flocculooculomotor tract of Wallenberg-Klimoff (Klimoff, 1899) produces a hypotonicity of upward gaze, often mistaken for a paresis. Irritative lesions may be indicated by a vertical nystagmus. These defects in upward gaze are a sign of cerebellar hemisphere or superior cerebellar peduncle involvement.

The efferent components of the superior cerebellar peduncle arise chiefly from nucleus interpositus of the monkey (nuclei globosus and emboliformis of man) and the lateral or dentate nucleus (fig. 63). In small part, they have origin also from the fastigial nucleus, for a small uncrossed fastigio-periventricular tract has been described by Carrea and Mettler (' 55 ) as emerging from the superior peduncle and distributing to reticular gray near to aqueduct.

The crossed descending cercbello-olivary tract arises (fig. 57) from the lateral or dentate nucleus and part of the interpositus nucleus. The bundle decussates in the brain stem, shortly after it leaves the superior cerebellar peduncle, and then divides into ascending and descending branches. Some of the fibers end in the tegmental or reticular gray of the brain stem (Carpenter and Nova, "60, for cat). Some have been followed through the medial longitudinal fasciculus to the eye muscle nuclei (Carrea and Mettler, '47) and perhaps some reach nucleus ambiguus (Bebin, '56). The major distribution of the path appears to be to the inferior olivary nucleus (Rand, '54). Certain fibers are said to extend beyond the nucleus into the cervical (Ramón y Cajal, '03) or even to the sacral cord (Orioli and Mettler, '56). In its course through the brain stem on its way to the inferior olivary nucleus, the tract adjoins the fascicles of the central tegmental bundle (Bebin, '56). Palatal nystagmus (Alajouanine, Thurel and Hornet, '35; Foix, Chavany and Hillemand, '26; Garcin, Bertrand and Frumusan, "33; Guillain, Thurel and Bertrand, ' 33 ; and others) is said to be produced by lesions of the combined bundle (Bebin, '56).

Decussating and non-decussating cerebellotegmental and crossed cerebellorubral and cerebellothalamic (or dentatothalamic) fibers (figs. 57, 64) make up the so-called crossed ascending limb of the brachium conjunctivum or superior cerebellar peduncle. The fibers to the red nucleus and the thalamus have been described by practically all those who have studied the peduncle extensively (Jakob, '28; Crouch and Thompson, '38; Jansen and Brodal, '54, '58; Carrea and Mettler, '54; Rand, '54; and a great many other observers). Fibers arising from the dentate distribute to the contralateral nucleus ventralis lateralis. Fascicles from the magnocellular (dorsolateral or dorsomedial) portion of the dentate and from the nucleus interpositus cross to the large-celled part of the red nucleus. The small-celled part of this nucleus receives fibers from the contralateral dentate nucleus. Impulses thus reaching the small-celled part of the red nucleus are relayed over rubrothalamic fibers to the nucleus ventralis lateralis (Rand, '54; Jansen and Brodal, '54, '58; and various others).

Some observers have described an uncrossed cerebellothalamic path ascending through the 


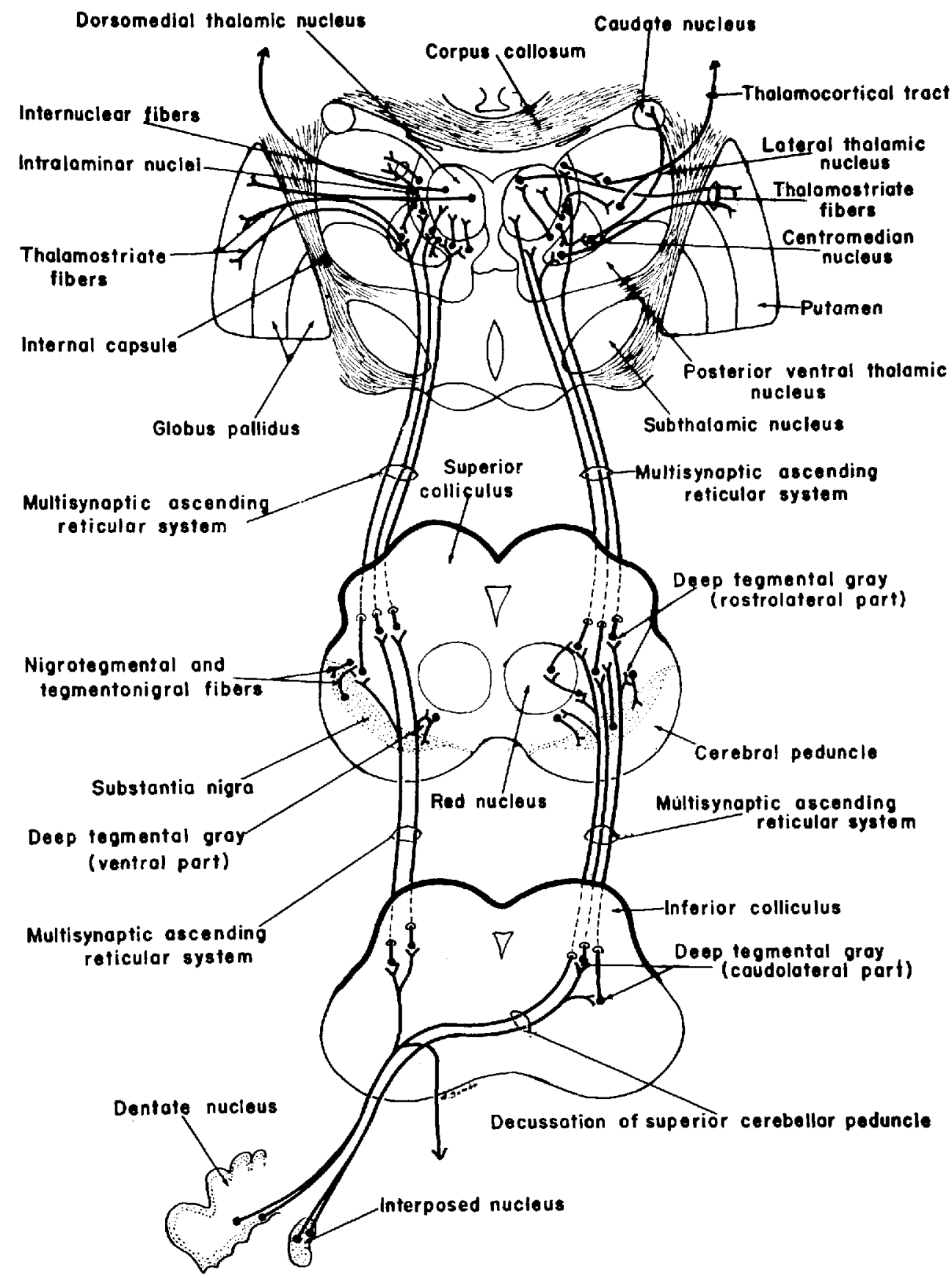

Fig. 64 Diagram to show multisynaptic ascending pathways from cerebellum and reticular gray by way of the intralaminar nuclei to other dorsal thalamic centers for relay to the extrapyramidal areas. 
brain stem, Iateral to the medial Iongitudinal fasciculus, to reach the nucleus ventralis lateralis (Carrea and Mettler, '54). This tract has not been documented by others who have searched for it (Hassler, '59). In the cat, according to Brodal and Gogstad ('54), fibers from the red nucleus to the cerebellum, which undergo a partial decussation in the superior cerebellar peduncle and enter the cerebellum by way of this peduncle, terminate primarily in the dentate nucleus but end, in part, in nucleus interpositus.

Impulses reaching the midbrain tegmental gray over cerebellotegmental fibers which end partly homolaterally and partly contralaterally, are relayed over tegmentothalamic or reticulothalamic fibers to the intralaminar nuclei. Ultimately such impulses reach the extrapyramidal or supplementary areas of the cortex. Such relay is probably not directly over the thalamocortical fibers (LeGros Clark and Russell, '39; see discussion and literature, Crosby, Humphrey and Lauer, '62, pp. 498-500) but indirectly by way of synapse in other nuclei of the dorsal thalamus.

\section{Cerebellar discharges to reticular gray}

The pathways reaching the reticular gray from the cerebellum directly (together with a certain amount of vestibuloreticular fascicles from the vestibular areas, with and without cerebellar inclusion in the arcs) terminate bilaterally on the cells of origin of reticulospinal or tegmentospinal tracts. Those to the red nucleus end, in part, on the cells of origin of a primarily crossed rubrobulbospinal tract which scarcely passes through the cervical cord in man. Those fascicles reaching chiefly the contralateral olivary complex relay with olivospinal fibers, the caudal extent of which may vary in the different mammals (Jansen and Brodal, '54). The cerebellar discharge to these various centers is probably in part facilitatory and in part inhibitory with the difference in effect depending upon the region of the cerebellum from which the impulses arise and perhaps upon the part (axons, dendrites, cell bodies) of the bulbar neurons on which the cerebellar pathways end.

There is abundant evidence that some reticular centers of the brain stem, including those of the tegmental region of the midbrain, have facilitatory and other reticular centers inhibitory effects on spinal cord neurons (Magoun, '44, '50; Magoun and Rhines, '46, '47; Niemer and Magoun, '47; Sprague et al., '48; Granit and Kaada, '53; Granit, '55; Brodal, '56; Glees, '61; and many others). Thus Granit and Kaada ('53) found that stimulation of the reticular gray of the brain stem, including that of the midbrain, accelerated the spindle discharge in the cat and maintained it, for half a minute or less, after the stimulation was no longer carried on. In decerebrate or anesthetized animals, a stimulation of the medial reticular gray inhibited or suppressed reflexes and had a similar effect, in anesthetized animals, on responses aroused by activation of the motor cortex (Magoun, '44; Magoun and Rhines, '46). A generalized suppression or inhibition of cortical motor responses, of multineuron flexor responses, and of tendon or two-neuron stretch responses were said to occur. Stimuli of ordinary intensity gave bilateral effects; very weak stimuli produced ipsilateral effects. Stimulation farther lateralward in the bulb and along an area extending forward into the midbrain (a much more extensive area) to involve the tegmentum, the central gray and the subthalamic area (Rhines and Magoun, '46) increased repsonses to stimulation of the motor cortex and facilitated the knee jerk. The suppressor functions are believed to be mediated over reticulospinal systems coursing through the ventrolateral funiculus of the cord and terminating primarily on the side of their origin. However, it is rare to have only inhibitory or only excitatory effects upon Iower motor neuron centers from a stimulation of reticular centers, for the discharges from these centers, like the spinal refiexes, are in accordance with Sherrington's law of reciprocal innervation (Ruch et al., '61). Contraction of the extensors of a limb occurs along with a relaxation of the appropriate flexors and the flexion of a limb is also accompanied by the relaxation of the opposing extensors (Sprague and Chambers, '54).

\section{B. Resumé of results of experimental cerebellar lesions}

Total or near total cerebellectomies resulted in a completely handicapping hypotonicity, so great that the monkeys were unable to stand. This disability appeared to be premanent; at least it persisted until the animals were sacrificed, sometimes as much as six months after the operation unless further procedures were carried out which changed the postoperative picture. One monkey with a complete section of one superior cerebellar peduncle lay on his side for slightly over six months after the initial operation. He pulled himself around the cage with his unaffected arm but was unable to stand on four legs until a subtotal removal of the cerebral cortex, contralateral to the section of the superior cerebellar peduncle, gave him a better balance.

Removal of the cortex of the posterior Iobe of the macaque cerebellum gave an incapacitating hypotonicity, although it was not so great as that seen after a cerebellectomy. Following removal of the posterior vermis, a monkey had a wide-based gait and there was a backward tilting of his head. A nystagmus might, or might not, be demonstrable, depending upon the exact extent of the lesion. There was usually a mild hypotonicity. If the lesions were unequal on the two sides (and particularly if one fastigial nucleus or the cerebellofastigial fibers on one side were more involved than those on the opposite side) 
a cerebellar nystagmus with a quick component toward the side of the lesion developed. There appeared to be a further decreased tonus, at least contralaterally, when the caudal part of the fastigial nucleus was destroyed. However, the experiments discussed in this paper were not set up to study the effects of lesions in the cerebellar nuclei without cerebellar cortex destruction. Consequently no informed opinion upon the effects of the destruction of parts of the fastigial nucleus can be offered. Small lesions of the vermis or the hemisphere of the posterior lobe produced no observed effects on tonus in the monkeys here reported. It would appear, then, that in the normal monkey, the cerebellar discharge augments tonus by discharging to (probably lateral) reticular centers which give rise to tegmentospinal paths with facilitatory effects over the gamma neurons.

Section through the brain stem at upper midbrain levels produces an animal or a child with a decerebrate rigidity. Following such a lesion, the cerebellar discharges are uninhibited by impulses from higher centers, and the results indicate that the activation of the gamma neurons by way of cerebello-reticular and tegmental paths and then reticulospinal bundles provides particularly for extension of the extremities. It seems likely that some of the cerebello-reticular fibers inhibit the gamma discharges to the antagonistic muscles (such as the flexors) either by inhibiting the impulses at reticular or gamma levels or more probably at both levels. Inhibition of the gamma neurons may be, and apparently is, a function of part of the anterior lobe in some animals, such as the cat. Granit and Kaada ('53) reported that stimulation of the vermis part of the anterior lobe produced inhibition of the gamma neurons. Sherrington, quoted from Glees ('61), is said to have abolished decerebrate rigidity by stimulation of the anterior lobe. Chambers and Sprague ('51) and Sprague and Chambers ("53) demonstrated a homolateral extensor rigidity and a contralateral flexor rigidity following removal of the cortex of the anterior lobe in the cat. The monkeys here reported with lesions in the cortex of the anterior lobe - and some of these lesions were of considerable size - showed either no effect or, at most, an exceedingly transitory, slight increase in tonus. However, none of the monkeys in the present series had a complete removal of the cortex of the anterior lobe with no other cerebellar involvement. The difference may be due to a species variation such as a shifting forward of functions (cephalization) in the higher animals.

It is of interest in this connection that although the total or near total cerebellectomy gives a major hypotonicity, nevertheless the outstanding hypotonicity is occasionally broken by what appears to be a hypertonic response (see also Manni and Dow, '63, for the rat). This response usually occurred when the monkey was annoyed, or emotionally upset, or perhaps just feeling uncooperative. Such a hypertonic response was of very short duration. Although the animals showed generalized hypotonicity from large posterior cerebellar lobe or superior peduncle lesions (Poirier, '60), loss of tonus was more marked in the lower than in the upper extremities. A monkey with an essentially complete cerebellectomy was able to prop up the front end of his body slightly with his arms. Otherwise he was prone.

\section{Postural tonus at basal ganglion levels}

In addition to its interrelations caudalward with the various reflex levels of the spinal cord and probably of the brain stem, the cerebellum also projects impulses rostralward to the dorsal thalamus. There are two main avenues of approach to the dorsal thalamus. One of these is over the dentatothalamic and dentatorubrothalamic paths (fig. 57) so frequently described (p. 73), which terminate in the thalamic nucleus ventralis lateralis on the side contralateral to their cerebellar origin. This thalamic nucleus discharges to motor and premotor areas (Walker, '36, '38). The other approach to the dorsal thalamus is by way of cerebellotegmental and tegmentothalamic fascicles, which constitute (fig. 64) a multisynaptic ascending path to the intralaminar nuclei of the dorsal thalamus (Crosby, Humphrey and Showers, '59). The intralaminar nuclei relay to adjacent nuclei of the dorsal thalamus (such as the dorsomedial and the lateral nuclei) which, 
in turn, project impulses to various cortical association centers (and so to supplementary motor or extrapyramidal regions). Some of these thalamic nuclei [the magnocellular part of the dorsomedial nucleus (Sheps, 45 ) and the centromedian nucleus (Droogleever Fortuyn and Stefens, '51)] discharge to the basal ganglia. Consequently cerebellar ablations or stimulations ultimately affect the efferent discharges not only from the supplementary motor and the motor areas of the cerebral cortex but also from the lenticular nuclei.

Another multisynaptic path over which impulses from the cerebellum reach the basal ganglia is by way of the substantia nigra. Cerebellotegmental fibers relay through tegmentonigral neurons to the cells of origin of the nigrostriate bundle.

The nigrostriate bundle (fig. 65), known to earlier observers as the comb bundle, has been described repeatedly (Ferraro, '28; Ranson and Ranson, '41; Kimmel, '42; Fox and Schmitz, '44; Rosegay, '44; Johnson and Clemente, '59). This fiber system, which is very important in the consideration of tonus, synapses in the putamen and probably in the globus pallidus after interdigitating with fascicles of the posterior limb of the internal capsule.

Destructive lesions in the lenticular nuclei tend to produce rigidity and characteristic changes in posture, with the particular type of postural change and the degree of rigidity depending upon the precise extent of the lesion( $s$ ) and the fiber systems and neurons involved (Bucy, '42a; Grinker, Bucy and Sahs, '60; DeJong, '58; DennyBrown, '62). In the uninjured brain, then, the lenticular discharge should tend to lessen or inhibit extensor tonus.

There is a tremendous amount of important experimental and clinical data dealing with the effects of lesions of the basal ganglia on the production of abnormal postures, rigidity, and abnormal movements. However, the monkeys in the present series had no involvements of the basal ganglia except in conjunction with large cortical lesions. Their relations as parts of discharge paths from supplementary motor areas have been discussed ( $p$. 65 ). It remains to tie them in as part of the balancing mechanism between the cerebral cortex and the cerebellum. In the lenticular nucleus, the discharges from the substantia nigra, the dorsal thalamus, and probably the subthalamic nucleus are played against impulses relayed to this nuclear area over corticostriate fibers from the supplementary motor and the motor areas of the cerebral cortex. These corticostriate fibers are of bilateral origin, some of them having crossed in the corpus callosum. In the uninjured monkey, they interbalance to give the normal shifts in tonus and changes in posture which occur at rest and during movement. Injury to one or more of the centers from which these tracts arise, to the tracts in course, or to greater or lesser portions of the lenticular nuclei upsets the normal balance. The characteristic signs of such involvement or imbalance are often very evident and frequently very bizarre. Denny-Brown ('62, p. 50) pointed out that, although each major disease process that affects the basal ganglia "may be recognized by certain peculiarities of motor disturbance, each presents a spectrum of change of movement and posture." He also emphasized the importance of evaluating the effects of specific fiber path destruction, as well as of cellular degeneration, in assessing the interrelations between causes and effects in explaining differences in tonus and posture in various basal ganglion syndromes.

\section{Postural tonus at cerebral cortex levels}

As is evident from the experimental results reported, and can be documented from other experimental and clinical studies, both the primary motor cortices and the supplementary motor areas are related to the regulation of tonus. The anatomical evidence for the discharge paths from these areas has been considered earlier in this discussion and need not be repeated here.

The destruction of area 4 or of its discharge path (the pyramidal tract) near its origin or in its course through the brain stem or in the spinal cord produced not only a loss of fine movements but, usually, a mild hypotonicity. Sometimes a monkey having a flaccid hemiparesis, as the result of destruction of this cortical area or its associated tract, showed increased deep reflexes.

A unilateral lesion in a single supplementary motor or extrapyramidal field 
usually did not alter tonus materially although occasionally it increased or decreased it slightly. A hemicortectomy produced no significant change in tonus in the monkeys studied. Small, bilateral cortical ablations may or may not effect slight transient increases in tonus. When such bilateral involvements become large, a

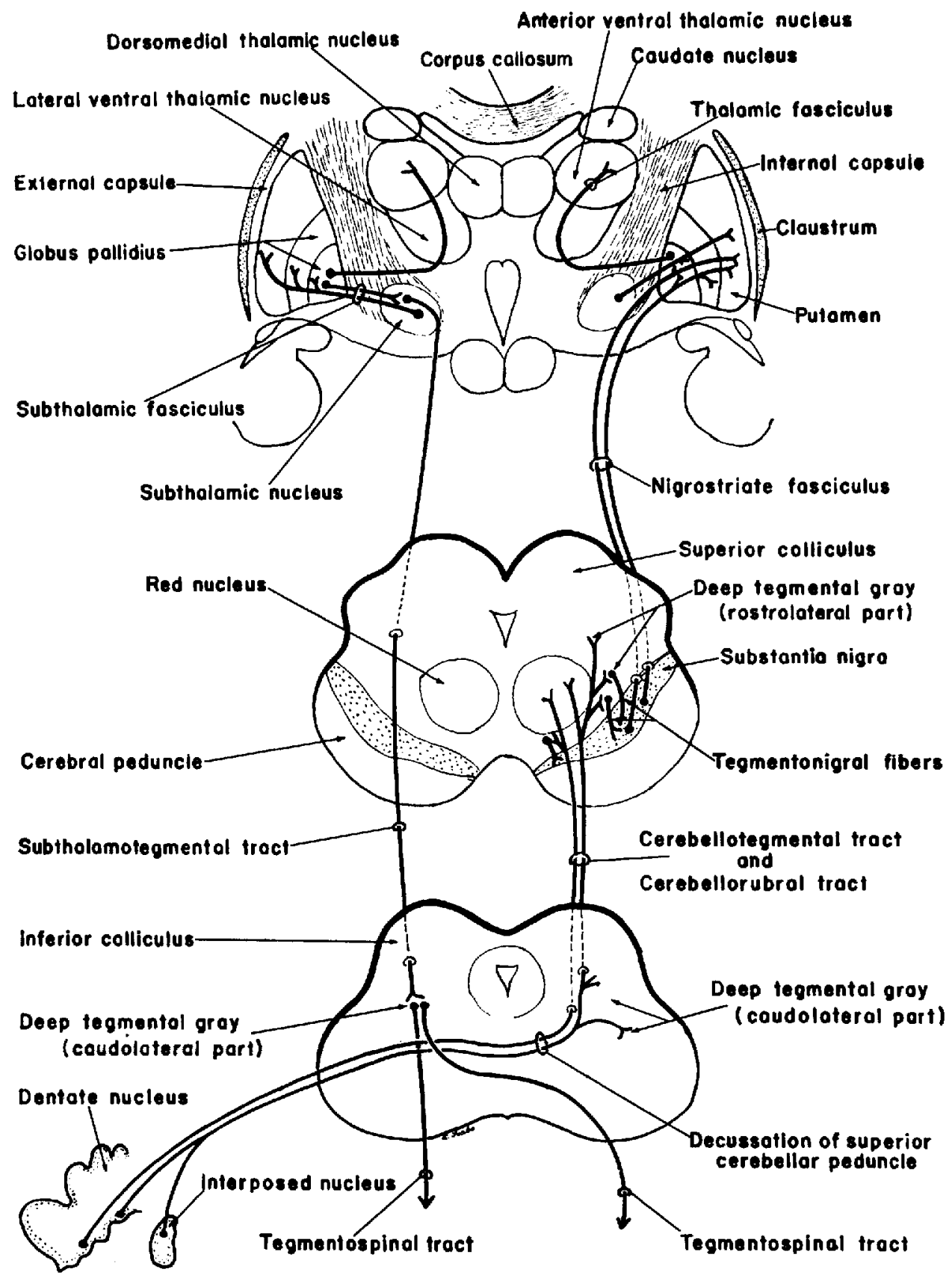

Fig. 65 Diagram showing particularly the cerebellonigrostriate system. 
hypertonicity does develop. If the cortex is totally or subtotally removed on the two hemispheres, an opisthotonos results. The animal has hyperextension of the back, the neck, and the lower extremities, but the arms are flexed at the elbows and the wrists and the fingers are often clenched. The tonus may be so rigid that the hands are drawn up near the face.

If considerable regions of the cortex are destroyed on one side, and the lenticular nucleus is also included, partly or wholly, in the lesion so that the corticostriate fibers of bilateral origin (since some have crossed in the corpus callosum from the other side) are included in the destroyed area then a hypertonicity develops. DennyBrown ('62) has pointed out that lesions of the putamen which include corticostriate fibers produce extension of the lower extremities and flexion of the upper extremities in man, a posture very comparable to that which the monkeys with bicortectomies showed (figs. 57, 62).

Involvement of the pyramidal tract together with the adjacent white matter, in the region overlying the internal capsule, will produce a contralateral spastic hemiplegia. Such deep white matter contains homolateral corticostriate fibers and also contralateral corticostriate fascicles which have crossed through the corpus callosum. Likewise, lesions of the pyramidal tract in its course in the internal capsule, where it is crossed by the discharge paths (lenticular fasciculus and subthalamic fasciculus ) of the basal ganglia, may be evidenced by a spastic hemiparesis. However, spasticity does not result from midbrain pedunculotomy (Walker, '52; and others), or bilateral section of the medullary pyramids (Tower, '49; and others) or the lateral corticospinal paths (Odeku, '65).

\section{Interplay between cerebellum and cerebral hemispheres \\ Experimental documentation}

The supplementary motor areas not only discharge through the basal ganglia, but many of them have direct corticotegmental paths (p. 59). Thus directly or indirectly these supplementary motor areas relay to the cells of origin of the brain stem tegmentospinal or reticulospinal systems, of the rubrospinal and the olivospinal tracts, and possibly of the vestibulospinal tracts. They reach, then, many of the same areas which are under the influence of the cerebellar discharge paths. From these brain stem centers there is a discharge to the alpha neurons for the production of body movements and to the gamma neurons for regulation of the tonus of these movements. Some of these discharges are inhibitory to, and some are excitatory to, the reticular centers and to the various other nuclei mentioned.

In the uninjured brain, it is difficult to see how there can be any cerebellar discharges which are not regulated from higher centers at thalamic, cerebellar and/ or brain stem levels, or any cortical or basal ganglion activities that are not affected at cerebral cortex, thalamus (Carpenter and Hanna, '62) and/or brain stem regions by impulses from the cerebellum. There is a constant interplay (occuring at many brain levels) for the regulation of tonus and the maintenance of normal posture between the cerebral hemispheres on the one hand and the cerebellum on the other.

Motor responses that are meaningful are initiated in the association areas of the cerebral cortex. There is discharge from these association areas (and perhaps in the highest types of activity particularly from the frontal association areas) to the motor cortex for activation of the pyramidal system which subserves the specific voluntary movements. There is also discharge to various supplementary motor areas for setting up impulses which give rise to the cortical automatic associated movements (often highly characteristic of the individual) that accompany such voluntary responses. The impulses are relayed also over corticopontocerebellar systems (figs. 66, 67, 68) to the cerebellum to regulate the cerebellar-cerebral hemisphere interplay at various brain levels, so that the degree of tonus and the coordination of responses advantageous to the contemplated motor performance may be obtained. Destruction of corticopontocerebellar tracts (or the frontal or the parietal areas from which many of them arise) produces an ataxia similar to that seen in cerebellar lesions.

Frontopontine fibers have been described by Sunderland ('40), Nyby and Jansen ('51), Jansen 

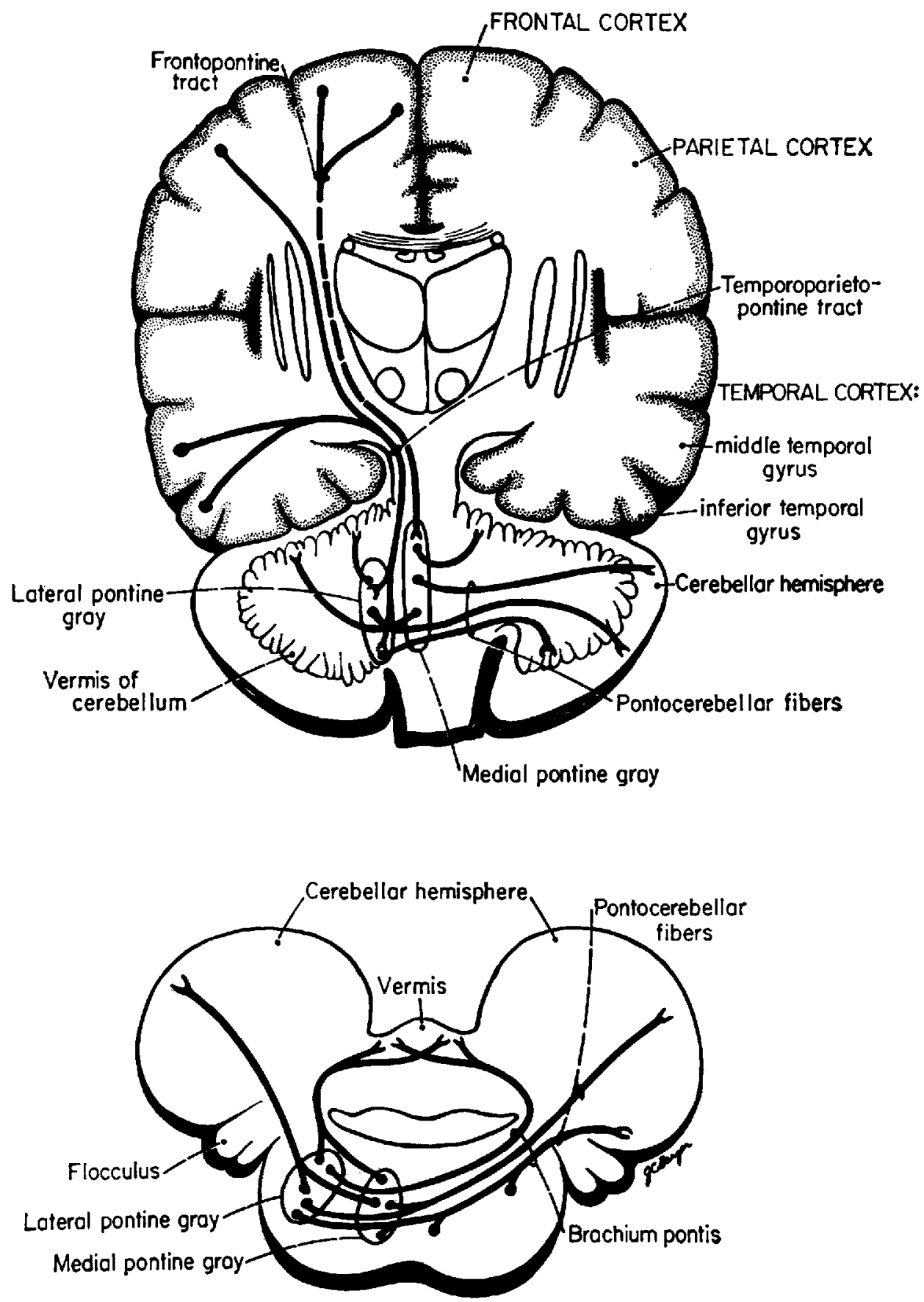

Fig. 66 Diagrams to show the corticopontine and pontocerebellar circuits. The bilateral projection to the cerebellum with emphasis on the crossed paths is to be noted. This figure and figure 67 are reproduced from portions of figure 162 from the Correlative Anatomy of the Nervous System ('62) by Crosby, Humphrey and Lauer. (Courtesy of The Macmillan Company, New York.) 


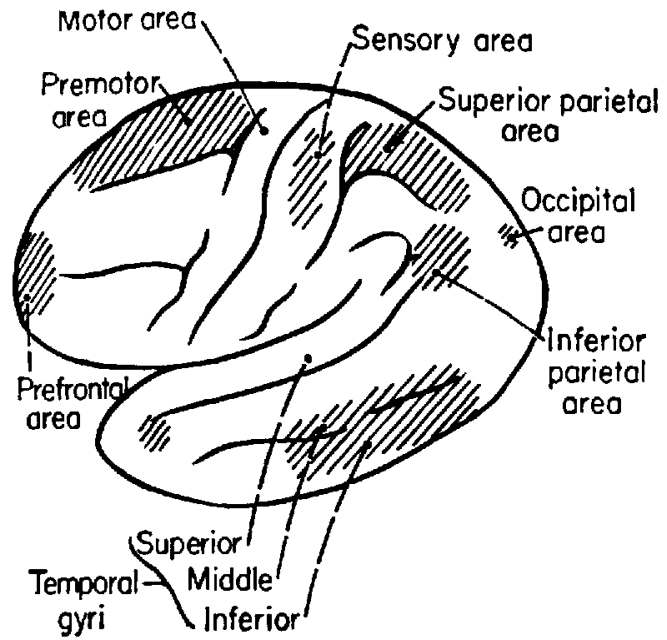

Fig. 67 Diagram to illustrate the cortical areas giving rise to the frontopontine, parieto(occipito)pontine, and temporopontine paths. (For acknowledgments see description under figure 66.)

and Brodal ('54, "58), and others. Parietopontine fibers (fig. 68), which are large in the macaque, have not always been recognized in man. Temporopontine fibers have been widely identified (the Dejerines, 1895, 01; Winkelman and Eckel, '29; Poirier, '52). A small occipitopontine tract is said to be present (Jansen and Brodal, '54, '58).

Impulses from the supplementary motor areas to tegmental or reticular areas of the brain stem by corticotegmental paths and by way of discharge through the basal ganglia interplay with cerebellar discharges to the same regions to produce adequate balance between flexion and extension for maintaining normal posture. In many individuals with widespread, bilateral cortical involvements, or massive cerebellar lesions, the tonus, whether increased or decreased, is so limited in range as to make normal posture or adequate motor performance impossible.

Complete section through the brain at the rostral end of the midbrain in man and in monkey (but somewhat farther caudalward in various other mammals) produces a decerebrate individual. Animals, or man, with such a lesion have a decerebrate rigidity in which there is a maximum extensor tonus. Here one is dealing with a cerebellar discharge uninhibited by cerebral hemisphere balancing. Animals (and probably man) with total cerebellectomies have a very great hypotonicity. Certainly under such conditions, the cerebral hemisphere discharges are not balanced by those from the cerebellum. Monkeys with a total removal of the cerebral cortex, but little or no involvements of the basal ganglia, show a hyperextension

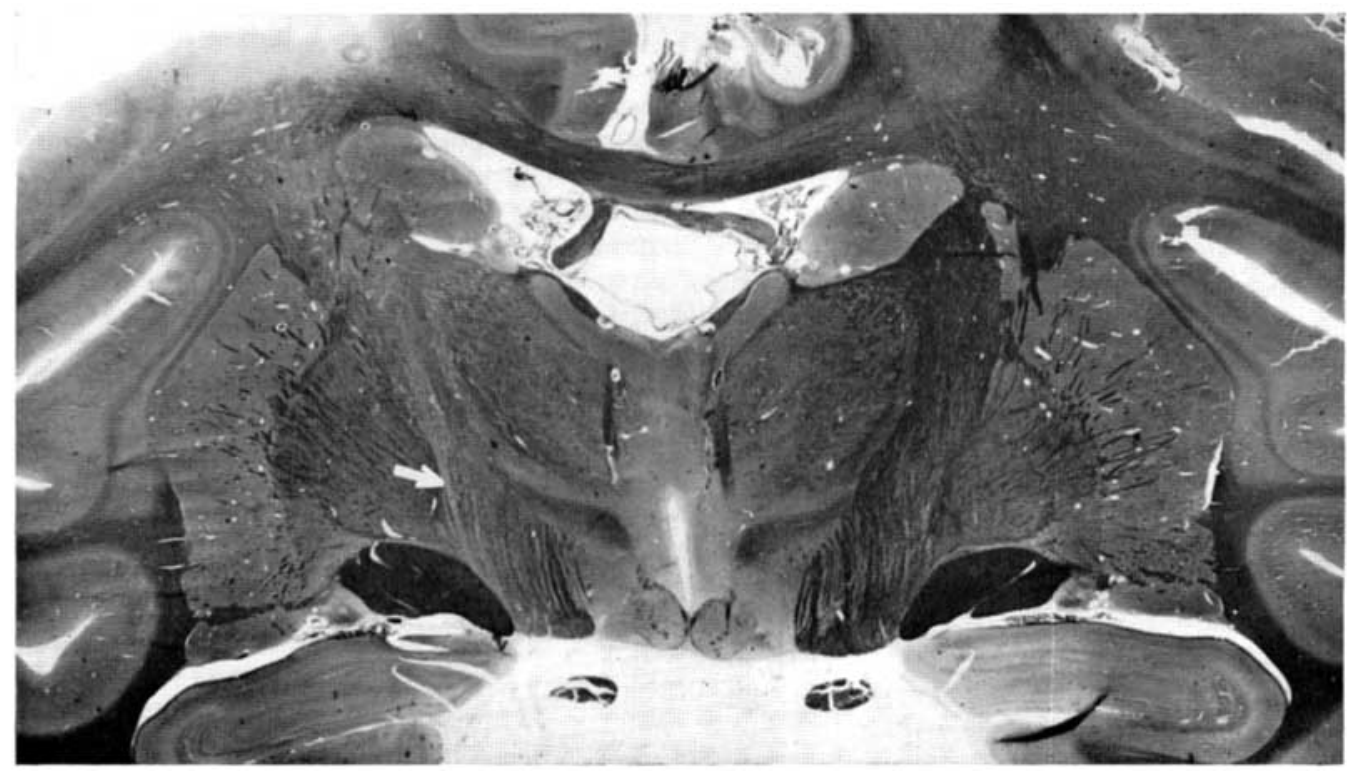

Fig. 68 Degeneration in the parietopontine path (arrow) following an area 5 lesion in the monkey. Weil preparation. 
of the lower extremities, the back, and the neck, and a rigid flexion of the upper extremities. It is interesting to compare the effects produced by decerebration with those produced by bicortectomies, where there is sparing of arcs from the thalamus through the basal ganglia to lower centers.

\section{Clinical documentation}

The operations on the cerebellum in children for the relief of intense hypertonicity were all undertaken after other methods of alleviating the condition had failed. With two exceptions they were carried out on subnormal children, whose mental tests suggested a wide involvement of association areas of the cortex (and so supplementary motor areas) as well as of motor areas. The other two children had dystonia musculorum deformans. The operation had three objectives in mind; (a) to make the children easier to care for at home, (b) to give them a little chance for some simple enjoyment in life; and (c) to relieve the intense pain which at least two of them found almost unendurable because of muscle spasms.

All the children who survived the operation showed a decreased tonus postoperatively. One child had a dislocated hip and his leg has remained extended. One child had more effect on one side than the other - the lesion, an early one, was unequal on the two sides. In the cerebral palsy children the results were apparently permanent, in fact there has been a slow improvement over a long period of time. This is undoubtedly due to the fact that the original defect in the cerebral cortex did not change and so the new balance established between the cerebral hemispheres and the cerebellum after the operation remained relatively adequate. Dystonia musculorum deformans is a progressive disease, presumably of the basal ganglia, and even though a satisfactory tonus may be achieved at some stage of the disease by an operation on the cerebellum (or the dorsal thalamus), the marked hypertonicity may appear again, as the lesions in the cerebral hemispheres increase. In such an event, the regulatory effects of the discharges of the cerebral hemispheres over those of the cerebellum are lessened. The adequate interplay between the two areas is upset and the overbalance of the cerebellar discharges produces the hypertonicity.

Children with intense hypertonicity may have muscle spasms which cause almost unbearable pain. In two children, pain of this origin has been relieved by reducing the hypertonicity through a partial cerebellectomy.

Neurosurgeons have undertaken various operations on human patients with a view to mitigating or banishing the hypertonicity and the involuntary movements due to major destruction of the cerebral hemispheres. Among such operations may be mentioned the ablation of cortical areas (Bucy, '42b, '49), the pallidectomies and the thalamotomies (Meyers, '42, '51, '58; Spiegel and Wycis, '52; Cooper, '56a, b, '59, '60; Bertrand et al., '60), the section of the ansa lenticularis (Meyers, '40), destructive lesions in the subthalamic area (Andy et al., '63) or in the zona incerta (the campotomy of Spiegel et al., '63) or in the substantia nigra (Meyers et al., '59), and the removal of portions of the cerebellum as described in this paper and elsewhere (Schneider and Crosby, '60, '63a, b).

It is obvious that all the operations just mentioned have a common basis. They all interfere with the paths or centers which interrelate impulses from supplementary motor areas with those from the cerebellum. They all have a common disadvantage in that they increase the lesions in an already injured brain. Those procedures which require invasions (especially bilateral invasions) of the cerebral cortex for placing the lesions (such as the thalamotomies) may, and, in some cases, have produced mental deficits. The destruction of the substantia nigra by ultrasonic waves invites the danger of a quadriplegia. The cerebellar operation may result in too great a hypotonicity. It is not the purpose here to advocate or to disparage any of the these operations. Possibly any one of them is valuable at the proper time and under proper conditions. The operations on patients referred to in the present paper have been used to illustrate the interpretations presented.

In closing this section on the discussion of tonus it may be pointed out that the uninjured individual (monkey or man) has at his command a wide range of tonus 
with which to carry on adequately his various motor activities. An individual with a lesion in the centers or tracts related to this balancing system - whether the injury is in the cerebral cortex, the basal ganglia, the thalamus, the tegmentum of the midbrain or the cerebellum, or in any of the tracts interrelating these areas - has a limited range of tonus. Depending upon the site and the extent of the lesion, and whether or not it is bilateral, the range may be so narrowed - the tonus so great or so little - that great in. capacitation results.

Some data on tremor and involuntary movements have been slowly accumulating from the present study and related experimental work. This data will not be reported further than has been done in the preceding protocols and will not be discussed at this time. However, in view of the very brief accounts of the cerebellar operations performed on children, a few facts about their appearance in some cerebellar lesions are possibly worth recording.

All of the monkeys in the present series with sufficient invasion of one dentate nucleus or considerable invasion of one superior cerebellar peduncle developed a homolateral action tremor. The severity of the tremor and the part of the body showing it depended upon the precise location of the lesion responsible for it. If the lesions were bilateral, so also was the tremor and more than twice the strength of that produced by a one-sided injury. The monkeys with cerebellectomies had such violent tremors that they injured their faces from knocking them against the cage walls. They could not feed themselves. In one monkey with a cerebellectomy, bilateral ablation of the portions of area 4 concerned with trunk and extremity movements (but with sparing of the so-called face areas) resulted in a bilateral cessation of the tremor on the trunk and the extremities but the head tremor still remained although it was not so violent as earlier. It became somewhat less over a period of several months.

It is evident that any lesions in the cerebellum aimed to decrease postural tonus in patients must avoid destruction of the dentate nucleus. Possibly destruction of the globose and the emboliform nuclei should be avoided for the same reason, for destruction of the nucleus interpositus in the monkey gives tremor (Carrea and Mettler, '47). Destruction of the fastigial nuclei does not produce tremor but alters tonus. Moruzzi and Pompeiano ('56) obtained an abolition or lessening of crossed extensor rigidity (but some increase in ipsilateral rigidity) when the caudal pole of the fastigial nucleus was destroyed in the cat. There was more effect on the hindlimbs than on the forelimbs. An increase contralaterally (and a decrease homolaterally) in the tonus followed destruction of the rostral part of the nucleus.

\section{SUMMARY}

1. The material presented in this paper is based on the results obtained from an extensive series of experiments on monkeys and a small number of operations on children. The experiments on the monkeys fell into three general groups: (a) ablations of various areas of the cerebral cortex; (b) destructive lesions of the cerebellum or the superior cerebellar peduncle (s); (c) combined lesions of the cerebral cortex and the cerebellum. The lesions varied in amount from small ablations involving some special part of a cortical area to bicortectomies, and from a limited destruction of a part of one lobule of the vermis to essentially complete cerebellectomies.

2. Destruction of the primary motor cortex produced a hypotonicity and a permanent loss of such fine movements as flexing one finger independently.

3. Supplementary motor areas are widespread. Most of them are located in association areas of the cerebral cortex. They have bilateral, multisynaptic projection to motor centers of the brain stem and the spinal cord. Stimulation of the supplementary motor areas (and so of the association cortices of which they are efferent parts) reveals a motor pattern of response comparable to that which has been demonstrated by various observers on area 4 . Illustrations are given of association areas which do, and those which do not, give changes in motor responses if destroyed. Other evidences of injury to association areas (such as aphasias) are consonant with the location of the particular area. 
4. Monkeys with total or subtotal removal of the cortex from one cerebral hemisphere walked again and carried on various motor activities although they never regained their ability to perform fine movements. They had an approximately normal tone if the basal ganglia were not invaded. A child with a subtotal hemicortectomy also was able to walk and to attend school, and graduated from high school.

5. Monkeys with bilateral removal of the motor cortex also walked again. As the lesions were increased to include various supplementary motor areas bilaterally, hypertonicity developed. Monkeys with near total bilateral cortectomies, or a complete removal of the cortex of both hemispheres, showed an opisthotonos. In such a monkey, the legs were in extensor tonus, the trunk was hyperextended, the arms were often bent at the elbows and the wrists and the hands were clenched and sometimes held against the face.

6. The bicortectomies destroyed the association areas along with the supplementary motor areas, so that the monkeys on which such experiments had been performed were completely unintelligent. They had a cortical blindness (with a retention of light reflexes and possibly of blinking) and of course a loss of voluntary eye deviations and optokinetic responses. There was bilateral loss of positional sense. Loss of tactile discriminatory ability was not proven satisfactorily (although suggested by the behavior of some animals), but the recognition of gross tactile and painful stimuli remained. The monkey jerked at a sudden noise but apparently had no understanding of the meaning of the sound. He had lost fine movements and other types of voluntary responses and, in the absence of supplementary motor areas, had no substitution for them. He had lost cortical automatic associated movements; for example, he had an expressionless face. The significance of the association areas for all types of cortically aroused motor responses is discussed.

7. Such small lesions as were placed in the vermis or the hemisphere portions of the posterior lobe of the cerebellum produced no noticeable defects. The animal with large lesions in the posterior vermis (nodule, uvula, pyramis and tuber) walked with his legs far apart (that is, had a wide base), with the hindlegs more affected than the forelegs. His head was tilted back on his shoulders (the equivalent of the tendency of a child with a similar lesion to fall backward). The monkey somtimes had a nystagmus, if the lesion was unequal on the two sides. He often showed a mild hypotonicity.

8. Large unilateral lesions in the hemisphere portions of the posterior lobe (or in both the hemisphere and the vermis) resulted in marked hypotonicity on the side of the lesion. If it were deep enough to involve the dentate or the interposed nucleus, an action tremor appeared homolaterally. With dentate involvement, there was sometimes a hypotonicity of upward gaze or a vertical nystagmus.

9. Complete cerebellectomies produced a permanent, incapacitating hypotonicity. Such an animal lies prone on his stomach with his head slightly propped up by his arms. He can crawl but does so badly.

10. Partial section of one superior cerebellar peduncle from the dorsolateral side, at the level of the anterior medullary velum, produced a homolateral hypotonic lower extremity. Section of this peduncle ventromedially, by a ventricular lesion, resulted in a hypotonic upper extremity on the side of the cut. Complete section of one superior cerebellar peduncle at upper fourth ventricle levels gave marked homolateral hypotonicity and ataxia. The monkeys with such a complete section had a homolateral action tremor.

11. Having produced hypotonicity and action tremor with large lesions of the cerebellum or its superior peduncles, and hypertonicity with major bilateral destruction of the cerebral cortex, the two types of lesions were placed successively in a series of monkeys to test the interplay between the discharges between the two regions. Such interplay occurs at spinal cord, medulla, cerebellar, midbrain, thalamic, basal ganglion, and cortex levels, as the account of the fiber connections and the discussion of their interrelations indicate. This balancing is evident in animals with lesions at both cerebellar and cerebral hemisphere levels. Thus, a monkey unable to walk for several months because of a section of the 
superior cerebellar peduncle, regained an erect posture and even walked a little without support after the cerebral cortex contralateral to the cerebellar injury was largely removed. The marked hypertonicity so evident in monkeys with massive bilateral cerebral cortex ablations was lessened by large cerebellar lesions involving the posterior vermis and portions of the hemispheres of the posterior cerebellar lobes.

12. In several children with intensely incapacitating hypertonicity, as a result of bilateral lesions in the cerebral cortex and/or the basal ganglia, removal of a large portion of the posterior vermis and adjoining regions of the hemispheres (see text) lessened this intense hypertonicity. Postoperatively these children were able to carry on better than before - feed themselves, write, and, in one case at least, attend school. Two children with intense pain from muscle cramp as a result of the hypertonicity were entirely relieved of that type of pain. These cases are described and discussed in the text.

\section{LITERATURE CITED}

Adrian, E. D., and G. Moruzzi 1939 Impulses in the pyramidal tract. J. Physiol. (Lond.), 97 : 153-199.

Alajouanine, Th., R. Thurel and T. Hornet 1935 Un cas anatomo-clinique de myoclonies vélo-pharyngées et oculaires. Rev. neurol., 64: 853-872.

Andy, O. J., M. F. Jurko and F. R. Sias, Jr. 1963 Subthalamotomy in treatment of Parkinsonian tremor. J. Neurosurg., 20: 860-870.

Baldwin, M., L. L. Frost and C. D. Wood 1954 Investigation of the primate amygdala. Move ments of the face and jaws. Neurology (Minneap.), 4: 586-598.

- 1956 Investigation of the primate amygdala: movements of the face and jaws. 2. Effect of selective cortical ablation. Neurology (Minneap.), 6: 288-293.

Bebin, J. 1956 The central tegmental bundle. An anatomical and experimental study in the monkey. J. Comp. Neur., 105: 287-332.

Beevor, C. E., and V. Horsley 1890 An experimental investigation into the arrangement of the excitable fibres of the internal capsule of the bonnet monkey (Macacus sinicus). Phil. Trans. B, 181: 49-88.

Berke, J. J. 1960 The claustrum, the external capsule and the extreme capsule of Macaca mulatta. J. Comp. Neur., 115: 297-331.

Bertrand, C., N. Martinez, L. Poirier and C. Gauthier 1960 Une méthode de traitement chirurgical de la maladie de Parkinson. Un. méd. Canada, 89: 297-299.

Blakeslee, G. A., I. S. Freiman and S. E. Barrera 1938 The nucleus lateralis medullae. An experimental study of its anatomic connections in
Macacus rhesus. Arch. Neur. Psychiat. (Chicago), 39: 687-701.

Brodal, A. 1956 The Reticular Formation of the Brain Stem. Anatomical Aspects and Functional Correlations. Oliver and Boyd, Edinburgh; Charles C Thomas, Springfield, Ill, $87 \mathrm{pp}$.

- 1964 Anatomical organization and fiber connections of the vestibular nuclei. In: Neurological Aspects of Auditory and Vestibular disorders, pp. 107-145. Ed. by William S. Fields and Bob R. Alford. Charles C Thomas, Springfield, Ill.

Brodal, A., and P. A. Drabløs 1963 Two types of mossy fiber terminals in the cerebellum and their regional distribution. J. Comp. Neur., 121: 173-187.

Brodal, A., and A. C. Gogstad 1954 Rubrocerebellar connections. An experimental study in the cat. Anat. Rec., 118: 455-485.

Brodal, A., and B. Høivik 1964 Site and mode of termination of primary vestibulocerebellar fibres in the cat. An experimental study with silver impregnation methods. Arch. ital. Biol., 102: 1-21.

Brodal, A., and F. Walberg 1952 Ascending fibers in pyramidal tract of cat. Arch. Neurol. Psychiat. (Chicago), 68: 755--775.

Bruce, A. 1895 On the flocculus. Brain, 18: $227-230$.

Brucher, J. M. 1964 L'aire oculogyre frontale du singe. Ses fonctions et ses voies efférentes. Editions Arscia S. A., Bruxelles; Librairie Maloine, Paris, 302 pp.

Bucy, P. C. 1942a The neural mechanisms of athetosis and tremor. J. Neuropath. exp. Neurol., 1: 224-239.

1942b Cortical extirpation in the treatment of involuntary movements. Res. Publ. Assoc. nerv. ment. Dis., 21: 551-595.

1949 Relation to abnormal involuntary movements. In: The Precentral Motor Cortex, 2nd ed., pp. 395-408, ed. by P. C. Bucy. Univ. of Illinois Press, Urbana.

Carpenter, M. B., and G. R. Hanna 1962 Effects of thalamic lesions upon cerebellar dyskinesia in the rhesus monkey. J. Comp. Neur., 119: 127-147.

Carpenter, M. B., and H. R. Nova 1960 Descending division of the brachium conjunctivum in the cat: a cerebello-reticular system. J. Comp. Neur., 114: 295-305.

Carrea, R. M. E., and F. A. Mettler 1947 Physiologic consequences following extensive removals of the cerebellar cortex and deep cerebellar nuclei and effect of secondary cercbral ablations in the primate. J. Comp. Neur., 87: 169288.

1954 The anatomy of the primate brachium conjunctivum and associated structures. J. Comp. Neur., 101: 565-689.

1955 Function of the primate brachium conjunctivum and related structures. J. Comp. Neur., 102: 151-322.

Chambers, W. W., and C.-N. Liu 1957 Corticospinal tract of the cat. An attempt to correlate the pattern of degeneration with deficits in reflex acivity following neocortical lesions. $J$. Comp. Neur., 108: 23-55. 
1958 Cortico-spinal tract in monkey. Fed. Proc., 17: 24.

Chambers, W. W., and J. M. Sprague 1951 Differential effects of cerebellar anterior lobe cortex and fastigial nuclei on postural tonus in the cat. Science, 114: 324-325:

Cohen, D., W. W. Chambers and J. M. Sprague 1958 Experimental study of the efferent projections from the cerebellar nuclei to the brainstem of the cat. J. Comp. Neur., 109: 233-259.

Cooper, I. S. 1956a The Neurosurgical Alleviation of Parkinsonism. Charles $C$ Thomas, Springfield, Ill. 104 pp.

$1956 \mathrm{~b}$ An investigation of neurosurg. ical alleviation of Parkinsonism, chorea, athetosis and dystonia. Ann. intern. Med, 45: $381-392$

- 1959 Chemopallidectomy and chemothalamectomy for Parkinsonism and dystonia. Proc. roy. Soc. Med., 52: 47-60.

1960 Results of 1000 consecutive basal ganglia operations for Parkinsonism. Ann. intern. Med., 52: 483-499.

Cranmer, R. 1951 Nystagmus related to lesions of the central vestibular apparatus and the cerebellum. Ann. Otol. (St. Louis), 60: 186196.

Critchley, M. 1953 The Parietal Lobes. Edward Arnold and Co., London. $480 \mathrm{pp}$.

Crosby, E. C. 1953a Relations of brain centers to normal and abnormal eye movements in the horizontal plane. J. Comp. Neur., 99: 437-480.

— 1953b Nystagmus as a sign of central nervous system involvement. Ann. Otol. (St. Louis ), 62: 1117-1127.

1956 The role of the midbrain as a part of the discharge paths from higher centers. In: Progress in Neurobiology, pp. 217-231, ed. by J. Ariëns Kappers. Elsevier, Amsterdam. AIso Folia psychiat. neerl., Suppl. 2.

Crosby, E. C., and J. W. Henderson 1948 The mammalian midbrain and isthmus regions. II. Fiber connections of the superior colliculus. B. Pathways concerned in automatic eye movements. J. Comp. Neur., 88: 53-91.

Crosby, E. C., T. Humphrey and E. W. Lauer 1962 Correlative Anatomy of the Nervous System. Macmillan, New York. 731 pp.

Crosby, E. C., T. Humphrey and M. J. Showers 1959 Einige Anordnungen, Verbindungen und Funktionen der supplementären motorischen Rinden. In: Medizinische Grundlagenforschung, 2: 101-124, ed. by K. F. Bauer. Thieme, Stuttgart.

Crosby, E. C., R. E. Yoss and J. W. Henderson 1952 The mammalian midbrain and isthmus regions. Part II. The fiber connections. D. The pattern for eye movements on the frontal eye field and the discharge of specific portions of this field to and through midbrain levels. J. Comp. Neur., 97: 357-383.

Crouch, R. L., and J. K. Thompson 1938 Termination of the brachium conjunctivum in the thalamus of the macaque monkey. J. Comp. Neur., 69: 449-452.

Dejerine, J., and Mme. Dejerine-KIumpke 18951901 Anatomie des Centres Nerveux. J. Reuff et Cie, Paris. 2 vols.
DeJong, R. N. 1958 The Neurologic Examination. 2nd ed. Hoeber, New York. $1078 \mathrm{pp}$.

Denny-Brown, D. 1962 The Basal Ganglia and their Relation to Disorders of Movement. Oxford Neurological Monographs, ed. by W. Ritchie Russell. Oxford University Press, Oxford. $144 \mathrm{pp}$.

Diamond, I. T., and W. D. Neff 1957 Ablation of temporal cortex and discrimination of auditory patterns. J. Neurophysiol., 20; 300-315.

Dow, R. S. 1936 The fiber connections of the posterior parts of the cerebellum in the rat and cat. J. Comp. Neur., 63: 527-548.

1938 Efferent connections of the flocculonodular lobe in Macaca mulatta. J. Comp. Neur., 68: 297-305

Dow, R. S., and G. Moruzzi 1958 The Physiology and Pathology of the Cerebellum. Univ. of Minnesota Press, Minneapolis, $675 \mathrm{pp}$.

Droogleever Fortuyn, J., and R. Stefens 1951 On the anatomical relations of the intralaminar and midline cells of the thalamus. Electroenceph. clin. Neurophysiol., 3: 393-400.

Dusser de Barenne, J. G., H. W. Garol and W. S. McCulloch 1942 Physiological neuronography of the cortico-striatal connections. Res. Publ. Assoc. nerv. ment. Dis., 21; 246-266.

Eager, R. P. 1963 Efferent cortico-nuclear path ways in the cerebellum of the cat. J. Comp. Neur., 120: 81-103.

Eccles, J. C., P. Fatt and S. Landgren 1956a Central pathway for direct inhibitory action of impulses in largest afferent nerve fibres to muscle. J. Neurophysiol., 19: 75-98.

- 1956b The inhibitory pathway to motoneurones. In: Progress in Neurobiology, ed. by J. Ariëns Kappers, pp. 73-82. Elsevier, Amsterdam. Also Folia psychiat. neerl., Suppl. 2.

Ferraro, A. 1928 The connections of the pars suboculomotoria of the substantia nigra. Arch. Neurol. Psychiat. (Chicago), 19: 177-180.

Fleming, J. F. R., and E. C. Crosby 1955 The parietal lobe as an additional motor area. The motor effects of electrical stimulation and ablation of cortical areas 5 and 7 in monkeys. J Comp. Neur., 103: 485-512.

Foerster, O. 1931 The cerebral cortex in man. Lancet, 2: 309-312.

1936 Motorische Felder und Bahnen. Sensible corticale Felder. In: Handbuch der Neurologie, $6 ; 1-448$, ed. by 0 . Bumke and 0 . Foerster. Springer, Berlin.

Foix, C., J.-A. Chavany and P. Hillemand 1926 Le syndrome myoclonique de la calotte. Etude anatomo-clinique du nystagmus du voile et des myoclonies rythmiques associées, oculaires, faciales, etc. Rev. neurol., 1: 942-956.

Foix, C., and J. Nicolesco 1925 Les noyaux gris centraux et la région mésencéphalo-sousoptique. Masson, Paris. 581 pp.

Forel, A. 1877 Untersuchungen über die Haubenregion und ihre oberen Verknüpfungen im Gehirne des Menschen und einiger Säugethiere, mit Beiträgen zu den Methoden der Gehirnuntersuchung. Arch. Psychiat. Nervenkr., 7: $393-495$.

Fox, C. A., and J. W. Barnard 1957 A quantitative study of the Purkinje cell dendritic branch- 
lets and their relationship to afferent fibres. J. Anat. (Lond.), 91; 299-313.

Fox, C. A., and J. T. Schmitz 1944 The substantia nigra and the entopeduncular nucleus in the cat. J. Comp. Neur., 80: 323-334.

French, J. D., M. Verzeano, and H. W. Magoun 1953 A neural basis of the anesthetic state. Arch. Neurol. Psychiat. (Chicago), 69: 519529.

Frontera, J. G. 1955 Preliminary report on the results of electrical stimulation of the island of Reil in the macaque. Anat. Rec., 121: 296. 1956 Some results obtained by electrical stimulation of the cortex of the island of Reil in the brain of the monkey (Macaca mulatta). J. Comp. Neur., 105: 365-394.

Furstenberg, A. C. 1958 Evidence of laryngeal participation in emotional expression: its relation to hysterical aphonia. Ann. Otol. (St. Louis ), 67: 516-527.

Garcin, R., I. Bertrand and P. Frumusan 1933 Etude anatomo-clinique d'un cas de syndrome de Parinaud et de myoclonies rythmiques du voile du palais. Rev. neurol., 2; 812-820.

Glees, P. 1944 The anatomical basis of corticostriate connexions. J. Anat. (Lond.), 78: 47-51. 1961 Experimental Neurology. Clarendon Press, Oxford. $532 \mathrm{pp}$.

Glees, P., J. Cole and E. G. T. Liddell 1951 The motor cortex of the macaque. Acta psychiat. scand., suppl., 74: 207-210.

Goodman, D. C., R. E. Hallett and R. B. Welch 1963 Patterns of localization in the cerebellar corticonuclear projections of the albino rat. J. Comp. Neur., 121: 51-67.

Granit, R. 1955 Receptors and Sensory Perception. Yale Univ. Press, New Haven, Oxford Univ. Press, London, $369 \mathrm{pp}$.

Granit, R., C. Job and B. R. Kaada 1952 Activation of muscle spindles in pinna reflex. Acta physiol. scand., 27: 161-168.

Granit, R., and B. R. Kaada 1953 Influence of stimulation of central nervous structures on muscle spindles in cat. Acta physiol. scand., 27: 130-160.

Granit, R., and C. G. Phillips 1956 Excitatory and inhibitory processes acting upon individual Purkinje cells of the cerebellum in cats. J. Physiol. (Lond.), 133: 520-547.

Grinker, R. R., P. C. Bucy and A. L. Sahs 1960 Neurology, 5th ed., Charles C Thomas, Springfield, IIl. 1386 pp.

Grünbaum, A. S. F., and C. S. Sherrington 1901 Observations on the physiology of the cerebral cortex of some of the higher apes. Proc. roy. Soc. B, 69: 206-209.

Guillain, G., R. Thurel and I. Bertrand 1933 Examen anatomo-pathologique d'un cas de myoclonies vélo-pharyngo-oculo-diaphragmatiques, associées à des myoclonies squelettiques synchrones. Rev. neurol, 2: 801-812.

Hagbarth, K.-E., and D. I. B. Kerr 1954 Central influences on spinal afferent conduction. J. Neurophysiol., 17: 295-307.

Hassler, R. 1959 Anatomy of the thalamus. In: Introduction to Stereotaxis with an Atlas of the Human Brain, 1: 230-290, ed. by G. Schaltenbrand and P. Bailey. Thieme, Stuttgart.
Henderson, J. W., and E. C. Crosby 1952 An experimental study of optokinetic responses, Arch. Ophthal. (Chicago), 47: 43-54.

Hess, W. R., S. Bürgi and V. Bucher 1946 Motorische Funktion des Tektal- und Tegmentalgebietes. Mschr. Psychiat. Neurol., 112: 152.

Hines, M. 1937 The "motor" cortex. Johns Hopk. Hosp. Bull., 60: 313-336.

1942 The development and regression of reflexes, postures, and progression in the young macaque. Contr. Embryol. Carneg. Instn., 30: 153-209.

1943 Control of movements by the cerebral cortex in primates. Biol. Rev., 18: $1-31$.

Hirasawa, K., and K. Kariya 1936 über die kortikalen extrapyramidalen Fasern aus dem motorischen Rindenfeld (Area 4a,b,c) beim Affen (Macacus rhesus). Folia anat. jap., 14: 603-620.

Hirasawa, K., S. Okano and S. Kamio 1938 Beitrag zur Kenntnis über die corticalen extrapyramidalen Fasern aus der Area temporalis superior (Area 22) beim Affen. Ztschr. mikr.anat. Forsch., 44: 74-84.

Hoff, E. C., and H. F. Hoff 1934 Spinal terminations of the projection fibres from the motor cortex of primates. Brain, 57: 454-474.

Holmes, G., and W. P. May 1909 On the exact origin of the pyramidal tracts in man and other mammals. Brain, 32: 1-43.

Hunt, C. C., and S. W. Kuffler 1951a Further study of efferent small-nerve fibres to mammalian muscle spindles. Multiple spindle innervation and activity during contraction. J. Physiol. (Lond.), 113: 283-297.

$1951 \mathrm{~b}$ Stretch receptor discharges during muscle contraction. J. Physiol. (Lond.), 113: $298-315$.

Hunt, C. C., and A. S. Paintal 1958 Spinal reflex regulation of fusimotor neurones. J. Physiol. (Lond.), 143: 195-212.

Jakob, A. 1923 Die extrapyramidalen Erkrankungen mit besonderer Berücksichtigung der pathologisehen Anatomie und Histologie und der Pathophysiologie der Bewegungsstörungen. Monographien a.d. Gesamtgebiete d. Neurol. u. Psychiat., 37: 1-416.

1928 Das Kleinhirn. In: Handbuch der microskopischen Anatomie des Menschen, Bd. IV/1: 674-916, ed. by W. v. Möllendorff. Springer, Berlin.

Jampel, R. S. 1960 Convergence, divergence, pupillary reactions and accommodation of the eyes from faradic stimulation of the macaque brain. J. Comp. Neur., 115: 371-399.

Jansen, J., and A. Brodal 1940 Experimental studies on the intrinsic fibers of the cerebellum. II. The cortico-nuclear projection. J. Comp. Neur., 73: 267-321.

1942 Experimental studies on the intrinsic fibers of the cerebellum. The corticonuclear projection in the rabbit and the monkey (Macacus rhesus). Norske Vidensk,, Akad. Avh., I Math. nat. Kl., nr 3, pp. 1-50.

1954 Aspects of Cerebellar Anatomy. 1st ed. Johan Grundt Tatum, Oslo. 423 pp. 
1958 Das Kleinhirn. In: Handbuch der mikroskopischen Anatomie des Menschen. Bd. IV/1: ed. by W. v. Möllendorf and W. Bargmann. Springer, Berlin. 323 pp.

Jansen, J., and J. Jansen, Jr. 1955 On the efferent fibers of the cerebellar nuclei in the cat. J. Comp. Neur., 102: 607-632.

Johnson, T. N., and C. D, Clemente 1959 An experimental study of the fiber connections between the putamen, globus pallidus, ventral thalamus, and midbrain tegmentum in cat. J. Comp. Neur., 113: 83-102.

Kimmel, D. L. 1942 Nigrostriatal fibers in the cat. Anat. Rec., 82: 425.

Klimoff, J. 1899 Über die Leitungsbahnen des Kleinhirns. Arch. Anat. Physiol, anat. Abt., pp. 11-27.

Kuypers, H. G. J. M. 1960a Central cortical projections to motor and somato-sensory cell groups. Brain, 83: 161-184.

$1960 b$ Central cortical projections to motor, somatosensory and reticular cell groups. In: Structure and Function of the Cerebral Cortex, pp. 138-143, ed. by D. B. Tower and J. P. Schadé. Elsevier, Amsterdam.

Larsell, O. 1937 The cerebellum: a review and interpretation. Arch. Neurol. Psychiat. (Chicago), 38: 580-607.

Lassek, A. M. 1942 The pyramidal tract. The effect of pre- and postcentral cortical lesions on the fiber components of the pyramids in monkey. J. nerv. ment. Dis., 95: 721-729.

1954 The Pyramidal Tract: Its Status in Medicine. Charles C Thomas, Springfield, Ill. $166 \mathrm{pp}$.

Laursen, A. M. 1955 An experimental study of pathways from the basal ganglia, J. Comp. Neur., 102: 1-25.

Le Gros Clark, W. E., and D. W. C. Northfield 1937 The cortical projection of the pulvinar in the macaque monkey. Brain, 60: 126-142.

Le Gros Clark, W. E., and W. R. Russell 1939 Observations on the efferent connexions of the centre median nucleus. J. Anat. (Lond.), 73: $225-262$.

Leksell, L. 1945 The action potential and excitatory effects of the small ventral root fibres to skeletal muscle. Acta physiol. scand., 10 (suppl. XXXI): 9-84.

Lemmen, L. J. 1951 An anatomical and experimental study of temporal and occipital association areas. J. Comp. Neur., 95: 521-559.

Lemmen, L. J., J. S. Davis and L. L. Radnor 1959 Observations on stimulation of the human frontal eye field. J. Comp. Neur., 112: 163-168.

Levin, P. M. 1936 The efferent fibers of the frontal lobe of the monkey, Macaca mulatta. J. Comp. Neur., 63: 369-419.

1949 Efferent Fibers. In: The Precentral Motor Cortex, pp. 133-148, ed. by P. C. Bucy. Univ. Illinois Press, Urbana.

Liu, C. N., and W. W. Chambers 1964 An experimental study of the cortico-spinal system in the monkey (Macaca mulatta). The spinal pathways and preterminal distribution of degenerating fibers following discrete lesions of the pre- and postcentral gyri and bulbar pyramid. J. Comp. Neur., 123: 257-283.
Lloyd, D. P. C. 1943 Conduction and synaptic transmission of the reflex response to stretch in spinal cats. J. Neurophysiol., 6: 317-326. 1946 Facilitation and inhibition of spinal motoneurons. J. Neurophysiol., 9: 421438.

Lockard, I. 1948 Certain developmental relations and fiber connections of the triangular gyrus in primates. J. Comp. Neur., 89: 349-386.

Löwy, R. 1916 Ueber die Faseranatomie und Physiologie der Formatio vermicularis Cerebelli. Arb. neurol. Inst. Univ. Wien, 21: 359-382.

Magoun, H. W. 1944 Bulbar inhibition and facilitation of motor activity. Science, 100: $549-550$.

- 1950 Caudal and cephalic influences of the brain stem reticular formation. Physiol. Rev., 30: 459-474.

Magoun, H. W., and R. Rhines 1946 An inhibitory mechanism in the bulbar reticular formation. J. Neurophysiol., 9: 165-171.

1947 Spasticity: the Stretch-Reflex and Extrapyramidal Systems. Charles C Thomas, Springfield, Ill. 59 pp.

Manni, E., and R. S. Dow 1963 Some observations on the effects of cerebellectomy in the rat. J. Comp. Neur., 121: 189-194.

Mellus, E. L. 1899 Motor paths in the brain and cord of the monkey. J. nerv. ment. Dis., 26: $197-209$.

Meyers, R. 1940 Surgical procedure for postencephalitic tremor, with notes on the physiology of premotor fibers. Arch. Neurol. Psychiat. (Chicago), 44: 455-458.

1942 The modification of alternating tremors, rigidity and festination by surgery of the basal ganglia. Res. Publ. Assoc. nerv. ment. Dis., 21: 602-665.

1951 Surgical experiments in the therapy of certain "extrapyramidal" diseases: a current evaluation. Acta psychiat. et neurol. scand. (suppl.), 67: 1-42.

1958 Historical background and personal experiences in the surgical relief of hyperkinesia and hypertonus. In: Pathogenesis and Treatment of Parkinsonism, pp. 229-270, ed by W. S. Fields. Charles C Thomas, Springfield, Ill.

Meyers, R., W. J. Fry, F. J. Fry, L. L. Dreyer, D. F. Schultz and R. F. Noyes 1959 Early experiences with ultrasonic irradiation of the pallidofugal and nigral complexes in hyperkinetic and hypertonic disorders. J. Neurosurg., 16: 32-54.

Minkowski, M. 1923 Etude sur les connexions anatomiques des circonvolutions rolandiques, pariétales et frontales. Schweiz. Arch. Neurol. Psychiat., 12: 71-104, 227-268.

1924 Etude sur les connexions anatomiques des circonvolutions rolandiques, pariétales et frontales. Schweiz. Arch. Neurol. Psychiat., 14: 255-278; 15: 97-132.

Monakow, C. v. 1895 Experimentelle und pathologisch-anatomische Untersuchungen über die Haubenregion, den Sehhügel und die Regio subthalamica, nebst Beiträgen zur Kenntniss früh erworbener Gross- und Kleinhirndefecte. Arch. Psychiat. Nervenkr., 27: 1-128, 386-478.

Monrad-Krohn, G. H. 1924 On the dissociation of voluntary and emotional innervation in 
facial paresis of cortical origin. Brain, 47: 22-35.

Morgan, L. O. 1927 The corpus striatum. A study of secondary degenerations following lesions in man and of symptoms of acute degenerations following experimental lesions in cats. Arch. Neurol. Psychiat. (Chicago), 18: 495-549.

Moruzzi, G., and O. Pompeiano 1956 Crossed fastigial influence on decerebrate rigidity. $\mathrm{J}$. Comp. Neur., 106: 371-392.

Mott, F. W., and E. A. Schaefer 1890 On associated eye-movements produced by cortical faradization of the monkey's brain. Brain, 13: 165-173.

Neff, W. D., and I. T. Diamond 1958 The neural basis of auditory discrimination. In: Biological and biochemical bases of behavior, pp. 101-126, ed. by H. F. Harlow and C. N. Woolsey. Univ. Wisconsin Press, Madison.

Neumann, M. A. 1959 Combined degeneration of globus pallidus and dentate nucleus and their projections. Neurology (Minneap.), 9: $430-438$.

Nielsen, J. M. 1958 Memory and Amnesia. San Lucas Press, Los Angeles, Calif. 205 pp.

Niemer, W. T., and H. W. Magoun 1947 Reticulo-spinal tracts influencing motor activity. J. Comp. Neur., 87: 367-379.

Nyberg-Hansen, R., and A. Brodal 1963 Sites of termination of corticospinal fibers in the cat. An experimental study with silver impregnation methods. J. Comp. Neur., 120: 369-391.

Nyby, O., and J. Jansen 1951 An experimental investigation of the cortico-pontine projection in Macaca mulatta. Norske Vid. Akad. Avh. I. Math.-nat. Kl., no. 3, pp. 1-47.

Odeku, E. L. 1965 The effects on muscle tonus and strength of lesions of the macaque corticospinal tract at spinal cord levels. Univ. of Michigan Hosp. J., 31: 234-239.

Orioli, F. L., and F. A. Mettler 1956 Descending limb of the brachium conjunctivum in Macaca mulatta. J. Comp. Newr, 106: 339-362.

Papez, J. W. 1942 A summary of fiber connections of the basal ganglia with each other and with other portions of the brain. Res. Publ. Assoc. nerv. ment. Dis., 21: 21-68.

- 1946 Subthalamo-tegmental tract. Anat. Rec., 94: 524

Peele, T. L. 1942 Cytoarchitecture of individual parietal areas in the monkey (Macaca mulatta) and the distribution of the efferent fibers. J. Comp. Neur., 77: 693-737.

- 1944 Acute and chronic parietal lobe ablations in monkeys. J. Neurophysiol., 7: 269-286.

Penfield, W., and T. Rasmussen 1950 The Cerebral Cortex of Man. A Clinical Study of Localization of Function. Macmillan, New York. 248 pp.

Penfield, W., and K. Welch 1951 The supplementary motor area of the cerebral cortex. A clinical and experimental study. Arch. Neurol. Psychiat. (Chicago), 66: 289-317.

Poirier, L. J. 1952 Anatomical and experimental studies on the temporal lobe of the macaque. J. Comp. Neur., 96: 209-248.
1960 Experimental and histological y of midbrain dyskinesias. J. Neurophysiol., 23: 534-551.

Rae, A. S. L. 1954 The connections of the claustrum. Confin. neurol. (Basel), 14: 211219.

Ramón y Cajal, S. 1903 La doble via descendente. Nacida del pedúnculo cerebeloso superior. Trab. Inst. Cajal Invest. biol. (Madrid), 2: $23-29$

- 1909-11 Histologie du Système Nerveux de L'Homme et des Vertébrés. Maloine, Paris. 2 vols.

Rand, R. W. 1954 An anatomical and experimental study of the cerebellar nuclei and their efferent pathways in the monkey. J. Comp. Neur., 101: 167-223.

Ranson, S. W., and M. Ranson 1939 Pallidofugal fibers in the monkey. Arch. Neurol. Psychiat. (Chicago), 42: 1059-1067.

Ranson, S. W., and S. W. Ranson, Jr, 1941 Strionigral or nigrostriatal fibers. Trans. Amer. neurol. Assoc., pp. 168-171.

1942 Efferent fibers of the corpus striatum. Res. Publ. Assoc. nerv, ment. Dis., 21: $69-76$

Ranson, S. W., S. W. Ranson, Jr. and M. Ranson 1941 Fiber connections of corpus striatum as seen in Marchi preparations. Arch. Neurol. Psychiat. (Chicago), 46: 230-249.

Rasmussen, A. T. 1932 Secondary vestibular tracts in the cat. J. Comp. Neur, 54: 143-171.

Rasmussen, T., and W. Penfield 1947 Further studies of the sensory and motor cerebral cortex of man. Fed. Proc., 6: 452-460.

Renshaw, B. 1946 Central effects of centripetal impulses in axons of spinal ventral roots. J. Neurophysiol., 9: 191-204.

Rhines, R., and H. W. Magoun 1946 Brain stem facilitation of cortical motor response. J. Neurophysiol., 9: 219-229.

Rieck, N. W. 1959 Motor responses from the macaque occipital lobe. J. Comp. Neur., 112: 203-229.

Riley, H. A. 1943 An Atlas of the Basal Ganglia, Brain Stem and Spinal Cord. Williams and Wilkins, Baltimore. $708 \mathrm{pp}$.

Rosegay, H. 1944 An experimental investigation of the connections between the corpus striatum and substantia nigra in the cat. $J$. Comp. Neur., 80: 293-321.

Ruch, T. C., H. D. Patton, J. W. Woodbury and A. L. Towe 1961 Neurophysiology. W. B. Saunders Company, Philadelphia and London. $521 \mathrm{pp}$.

Scarff, J. F. 1940 Primary cortical centers for movements of upper and lower limbs in man. Arch. Neurol. Psychiat. (Chicago), 44: 243-299.

Schemm, G. W. 1961 The pattern of cortical localization following cranial nerve cross anastomosis. J. Neurosurg., 18: 593-596.

Schemm, G. W., and E. A. Kahn 1960 The effect of peripheral nerve cross anastomosis on the cortical localization pattern. Trans. Am. neurol. Assoc., pp. 225-226.

Scherrer, H., and R. Hernandés-Peón 1955 Inhibitory influences of reticular formation upon synaptic transmission in gracilis nucleus. Fed. Proc., 14: 132. 
Schneider, R. C., and E. C. Crosby 1954 Stimulation of "second" motor areas in the macaque temporal lobe. Neurology (Minneap.), 4: 612622.

1960 A study of shifts of tonus and motor dysfunctions from brain lesions in primates. In: Structure and Function of the Cerebral Cortex, pp. 104-115, ed. by D. B. Tower and J. P. Schadé. Elsevier, Amsterdam.

1963a Current clinical practice and applied research in neurological surgery in a medical center. J. Mich. State Med. Assoc., 62: 175-183.

1963b The interplay between cerebral hemispheres and cerebellum in relation to tonus and movements. J. Neurosurg., 20: 188-198.

Schneider, R. C., E. A. Kahn and E. C. Crosby 1951 Extradural hematoma of the posterior fossa. Neurology (Minneap.), 1: 386-393.

Segundo, J. P., R. Naquet and R. Arana 1955 Subcortical connections from temporal cortex of monkey. Arch. Neurol. Psychiat. (Chicago), 73: 515-524.

Sheps, J. G. 1945 The nuclear configuration and cortical connections of the human thalamus. J. Comp. Neur., 83: 1-56.

Sherrington, C. S. 1893 Further experimental note on the correlation of action of antagonistic muscles. Proc. roy. Soc. B, 53: 407-420.

Showers, M. J. C. 1959 The cingulate gyrus: additional motor area and cortical autonomic regulator. J. Comp. Neur., 112: 231-301.

Showers, M. J. C., and E. C. Crosby 1958 Somatic and visceral responses from the cingulate gyrus. Neurology (Minneap.), 8: 561-565.

Showers, M. J. C., and E. W. Lauer 1961 Somatovisceral motor patterns in the insula. J. Comp. Neur., 117: 107-115.

Smith, W. K. 1936 Ocular responses elicited by electrical stimulation of the cerebral cortex. Anat. Rec., 64 (suppl.): 45.

1949 The frontal eye fields. In: The Precentral Motor Cortex, pp. 307-342, ed. by P. C. Bucy, Univ. Illinois Press, Urbana.

Spiegel, E. A., and I. Sommer 1944 Neurology of the Eye, Ear, Nose, and Throat. Grune and Stratton, New York. $667 \mathrm{pp}$.

Spiegel, E. A., and H. T. Wycis 1952 Stereoancephalotomy (Thalamotomy and Related Procedures). Part I. Methods and Stereotaxic Atlas of the Human Brain. Grune and Stratton, New York. $176 \mathrm{pp}$.

Spiegel, E. A., H. T. Wycis, E. G. Szekely, J Adams, M. Flanagan and H. W. Baird, III 1963 Campotomy in various extrapyramidal disorders. J. Neurosurg., 20: 871-884.

Sprague, J. M., and W. W. Chambers 1953 Regulation of posture in intact and decerebrate cat. I. Cerebellum, reticular formation, vestibular nuclei. J. Neurophysiol., 16: 451-463.

1954 Control of posture by reticular formation and cerebellum in the intact, anesthetized and unanesthetized and in the decerebrated cat. Am. J. Physiol., 176: 52-64.

Sprague, J. M., L. H. Schreiner, D. B. Lind̄sley and $\mathrm{H}$. W. Magoun 1948 Reticulo-spinal influences on stretch reflexes. J. Neurophysiol., 11: 501-507.
Sugar, O., J. G. Chusid and J. D. French 1948 $A$ second motor cortex in the monkey (Macaca mulatta). J. Neuropath. exp. Neurol., 7: 182189.

Sunderland, S. 1940 The projection of the cerebral cortex on the pons and cerebellum in the macaque monkey. J. Anat. (Lond.), 74: 201-226.

Thomas, D. M., R. P. Kaufman, J. M. Sprague and W. W. Chambers 1956 Experimental studies of the vermal cerebellar projections in the brain stem of the cat (fastigiobulbar tract). J. Anat. (Lond.), 90: 371-385.

Tower, S. S. 1940 Pyramidal lesion in the monkey, Brain, 63: 36-90.

1949 The pyramidal tract. In: The Precentral Motor Cortex, pp. 149-172, ed. by P. C. Bucy. Univ, Illinois Press, Urbana.

Tower, S. S., and M. Hines 1935 Dissociation of the pyramidal and extrapyramidal functions of the frontal lobe. Science, 82: 376.

Travis, A. M. 1955 Neurological deficiencies following supplementary motor area lesions in Macaca mulatta. Brain, 78: 174-198.

Vachananda, B. 1959 The major spinal afferent systems to the cerebellum and the cerebellar corticonuclear connections in Macaca mulatta. J. Comp. Neur., 112: 303-351.

Verhaart, W. J. C., and M. A. Kennard 1940 Corticofugal degeneration following thermocoagulation of areas 4,6 , and 4-s in Macaca mulatta. J. Anat. (Lond.), 74: 239-254.

Vogt, C., and O. Vogt 1907 Zur Kenntnis der elektrisch-erregbaren Hirnrindengebiete bei den Säugetieren. J. Psychol. Neurol. (Lpz.)., 8: $277-456$.

Votaw, C. L. 1957 The hippocampus as a supplementary motor area. Anat. Rec., 127: 382383.

- 1959 Certain functional and anatom ical relations of the cornu Ammonis of the macaque monkey. I. Functional relations. J. Comp. Neur., 112: 353-382.

Walberg, F. 1955 Über die sogenannte "Zentrale Haubenbahn." Arch. Psychiat. Nervenkr., 193: 252-260.

1958 Descending connections to the lateral reticular nucleus. An experimental study in the cat. J. Comp. Neur., 109: 363389.

Walberg, F., and A. Brodal 1953 Pyramidal tract fibres from temporal and occipital lobes. An experimental study in the cat. Brain, 76; $491-508$.

Walberg, F., and J. Jansen 1961 Cerebellar corticovestibular fibers in the cat. Exper. Neurol., 3: 32-52.

1964 Cerebellar corticonuclear projection studied experimentally with silver impregnation methods. J. f. Hirnforschung, 6: 338354.

Walberg, F., and O. Pompeiano 1960 Fastigiofugal fibers to the lateral reticular nucleus: an experimental study in the cat. Exper. Neurol., 2: $40-53$

Walberg, F., O. Pompeiano, A. Brodal and J. Jansen 1962a The fastigiovestibular projection in the cat. An experimental study with 
silver impregnation methods. J. Comp. Neur, 118: 49-75.

Walberg, F., O. Pompeiano, L. E. Westrum and E. Hauglie-Hanssen 1962b Fastigioreticular fibers in the cat. An experimental study with silver methods. J. Comp. Neur., 119: 187-199.

Walker, A. E. 1936 An experimental study of the thalamocortical projection of the macaque monkey. J. Comp. Neur., 64: 1-39.

- 1938 The Primate Thalamus. Univ. of Chicago Press, Chicago. 321 pp.

- 1952 Cerebral pedunculotomy for the relief of involuntary movements. II. Parkinsonian tremor. J. nerv. ment. Dis., 116: 766775 .

Ward, A. A. 1948 The cingular gyrus: Area 24. J. Neurophysiol., 11: 13-23.

Whittier, J. R., and F. A. Mettler 1949a Studies on the subthalamus of the rhesus monkey. I. Anatomy and fiber connections of the subthalamic nucleus of Luys. J. Comp. Neur., 90: $281-317$.

_ 1949b Studies on the subthalamus of the rhesus monkey. II. Hyperkinesia and other physiologic effects of subthalamic lesions with special reference to the subthalamic nucleus of Luys. J. Comp. Neur., 90:319-372.

Wilson, S. A. K. 1914 An experimental research into the anatomy and physiology of the corpus striatum. Brain, 36: 427-492.

Winkelman, N. W., and J. L. Eckel 1929 Origin of the cortico-cerebellar system as determined in human pathological material. Res. Publ. Assoc. nerv. ment. Dis., 6: 481-493.

Woodburne, R. T., E. C. Crosby and R. E. McCotter 1946 The mammalian midbrain and isthmus regions, Part $\amalg$. The fiber connections. A. The relations of the tegmentum of the midbrain with the basal ganglia in Macaca mulatta. J. Comp. Neur., 85: 67-92.

Woolsey, C. N. 1938 Representation in the motor cortex of flexor and extensor muscles of the leg. Am. J. Physiol., 123 221-222.

1952 Patterns of localization in sensory and motor areas of the cerebral cortex. In: The Biology of Mental Health and Disease, 27th Ann. Conf., Milbank Memorial Fund, pp. 193-206. Hoeber, New York.

1958 Organization of somatic sensory and motor areas of the cerebral cortex. In: Biological and Biochemical Bases of Behavior, pp. 63-81, ed. by H. F. Harlow and C. N Woolsey. Univ. of Wisconsin Press, Madison.

Woolsey, C. N., and H.-T. Chang 1948 Activation of the cerebral cortex by antidromic volleys in the pyramidal tract. Res. Publ. Assoc. nerv. ment. Dis., 27: 146-161.

Woolsey, C. N., and P. H. Settlage 1950 The pattern of localization in the precentral motor cortex of Macaca mulatta. Fed. Proc., 9: 140.

woolsey, C. N., P. H. Settlage, D. R. Meyer, W. Sencer, T. P. Hamuy and A. M. Travis 1952 Patterns of localization in precentral and "supplementary" motor areas and their relation to the concept of a premotor area. Res. Publ. Assoc. nerv, ment. Dis., 30: 238-264.

Woolsey, C. N., P. H. Settlage, H. M. Suckle and W. G. Bingham 1949 The pattern of localization in the motor cortex of the marmoset (Hapale jacchus). Fed. Proc., 8: 172.

Woolsey, C. N., R. Tasker, C. Welt, R. Iadpli, G. Campos, H. D. Potter, R. Emmers and H. Schwassman 1960 Organization of pre- and postcentral leg areas in chimpanzee and gibbon. Trans. Amer. neurol. Assoc, pp. 144-146. 